\title{
Modeling and Observations of Massive Star Cluster Formation
}

\author{
David Graham Whelan \\ Charlottesville, VA \\ M.S., University of Virginia, 2009 \\ B.A., Ithaca College, 2005 \\ A Thesis presented to the Graduate Faculty \\ of the University of Virginia in Candidacy for the Degree of \\ Doctor of Philosophy
}

Department of Astronomy

University of Virginia

August, 2013 


\section{Abstract}

In the Universe's panoply of star forming environments, super star clusters stand out as the most impressive. Their high densities, masses, and gas pressures are the extremes. But because so few examples exist in the Milky Way, and most are observed in distant blue compact dwarf galaxies, merger overlap regions, and similarly turbulent environments, details about their formation and early evolution remain obscure unknowns.

In this dissertation, I outline my modeling efforts that provide useful observables to studies of both embedded and optically visible high mass star forming environments. New dust radiative transfer models provide a set of infrared colors that will help determine some basic envelope properties; their use will only be fully realized with the advent of the James Webb Space Telescope. Likewise, models of the molecular emission from the envelope sheds new light on our understanding of the geometry in these regions, and highlights the ways in which future Atacama Large Millimeter/submillimeter Array observations will shed light on super star cluster formation.

Observations of a superassociation in the SMC provide some intriguing new insights into the dust and molecular properties of giant Hir regions at low metallicity and in hard radiation fields. And finally, Hi recombination line models offer compelling evidence that line pumping by the stellar continuum in HiI regions, and the subsequent line fluorescence, while important for the interpretation of Hydrogen line fluxes, can easily be overestimated with low-resolution input spectra.

By modeling aspects of massive star cluster evolution from when they are deeply embedded in their natal molecular cloud until they are surrounded by an HiI region, I have touched upon some of the most important aspects relating their physical properties with their observable properties. Future observations and models are planned to continue these investigations and provide concrete answers to many of the questions that remain about massive cluster formation. 


\section{Acknowledgements}

In the fall semester of 2002, I was a music major at Ithaca College. When I handed in my astronomy final that semester, the professor asked me what my major was, and whether I had ever considered physics. Beth Clark Joseph is therefore most deserving of acknowledgement, because without her I would never have considered a path in astronomy.

The next year, a new professor was hired with whom I immediately began research on Herbig AeBe stars. Luke D. Keller introduced me to spectroscopy, and together we reduced some of the very first spectra off of the (then new) Spitzer Space Telescope's Infrared Spectrograph. In acknowledgement for such an apt introduction to the study of dust, I formally dedicate Chapter 4 of this thesis.

I did an REU with Spitzer data under James R. Houck in the summer of 2004, a position which secured me a job after graduation. Dr. Houck has the best work ethic of any astronomer I know, and I would like to acknowledge the role he played in instilling some of that integrity on me. The success of my REU is also down to a class I took with Joseph Harrington - thank you for teaching me IDL in Linux.

During my senior year and for the two years I worked at Cornell, my closest working companion was my overseer, Daniel Devost. His active interest in me made all the difference, and I still count him as a great friend and collaborator. My other friends and collaborators deserve acknowledgement as well, if for no other 
reason than they were so patient with me: Vianney Lebouteiller, Henrik Spoon, Don Barry, Jeronimo Bernard-Salas, and Peter Hall principle among them.

The University of Virginia has been an excellent training ground for me. My advisor, Kelsey Johnson, gave me relative freedom to conduct my own research and search out interesting new directions. All of the failed pathways, all of the steep learning curves, and finally all of the significant results, were the truest introduction to independent research possible. The other members of my thesis committee, Aaron Evans, Trinh X. Thuan, John T. Yates, and Fabian Heitsch, as well as the other astronomy faculty and NRAO scientists who made time for me outside the classroom, are equally deserving of respect in my book.

My best friend at UVa is George Privon. We have built a working relationship devoid of arrogant posturing, in which no question is too stupid and no inquiry is a waste of time. I look forward to tackling at least some of our many research ideas in the years to come.

Leslie, my wife, is the patientest soul and the most loving confidant. She has watched this whole process unfold, since its very inception eleven years ago, more closely than anybody. And from a distance, caring, loving, supportive, and understanding, have been my parents, Max and Joanna, my sister Alyson and her family, and my in-laws Harvey and Becky. They have all been immeasurably important. 


\section{Contents}

Abstract

$\begin{array}{ll}\text { Acknowledgements } & \text { ii }\end{array}$

1 An Introduction to Super Star Clusters and Applications of Conservation Laws Concerning their Formation 1

1.1 Observations of Super Star Cluster Environments . . . . . . . . . . 1

1.1.1 The Special Case of SBS 0335-052 . . . . . . . . . 5

1.1.2 What Questions Have Observations Left Unanswered? . . . 9

1.2 The Early Evolution of a Massive Star Cluster: Expectations from

Simple Analytical Arguments . . . . . . . . . . . . . . . . . . 11

1.2.1 Cloud Dispersal . . . . . . . . . . . . . . . . . . . 12

1.2.1.1 Fast GMC Dispersal . . . . . . . . . . . . . . 13

1.2.1.2 For Slow GMC Dispersal . . . . . . . . . . . . . 20

1.2.2 Taking Both Stellar Winds and Supernovae Into Account . . 21

1.2.3 Truly Realistic Models Treatment . . . . . . . . . . . . . . . 24

1.3 The Direction of this Thesis . . . . . . . . . . . . . . 25

2 The Infrared Properties of Embedded Super Star Clusters: Predictions from Three-Dimensional Dust Radiative Transfer Mod$\begin{array}{lr}\text { els } & 28\end{array}$

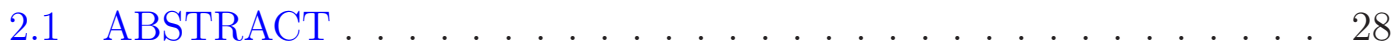

2.2 INTRODUCTION . . . . . . . . . . . . . . . . . 30

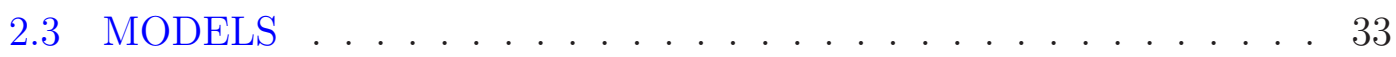

2.3.1 The Radiative Transfer Method . . . . . . . . . . . . . . 33

2.3.2 The Dust Composition . . . . . . . . . . . . . . 35

2.3.3 The Central Source . . . . . . . . . . . . . . 36

2.3.4 The Envelope Properties . . . . . . . . . . . . . 36

2.3.5 Supporting Observational Evidence for the Model Parameter Choices . . . . . . . . . . . . . . 40

2.4 RESULTS . . . . . . . . . . . . . . . . . . . . . 43 
2.4.1 Simulated Images . . . . . . . . . . . . . . . . 43

2.4.2 The Change in Ultraviolet Flux with Clumpy Dust Fraction 45

2.4.3 Infrared Variation with Viewing Angle . . . . . . . . . . . 47

2.4.4 Spectral Energy Distribution Properties . . . . . . . . . . 50

2.4.5 Infrared Colors as Tracers of the Input Parameters . . . . . 56

2.5 COMPARISONS TO OBSERVATIONS . . . . . . . . . . 65

2.6 KNOWN LIMITATIONS . . . . . . . . . . . . . . . . . . 67

2.7 SUMMARY . . . . . . . . . . . . . . . . . . . . . 69

3 First Models of the Molecular Emission from Embedded Super $\begin{array}{lr}\text { Star Clusters } & 71\end{array}$

3.1 ABSTRACT . . . . . . . . . . . . . . . . . 71

3.2 INTRODUCTION . . . . . . . . . . . . . . . . . . . 72

3.3 Chemical and Radiative Transfer Codes . . . . . . . . . . . . . . . 74

3.4 First Results: Computations Using Old Geometry . . . . . . . . . 75

3.5 A New Parametrization: New Envelope Geometries and Better Agreement with Expectations . . . . . . . . . . . . . . 81

3.6 Conclusions and Future Directions . . . . . . . . . . . . . . . . . 87

4 An In-Depth View of the Mid-Infrared Properties of Point Sources and the Diffuse ISM in the SMC Giant HiI Region, N66 90

4.1 Abstract . . . . . . . . . . . . . . . . . . 90

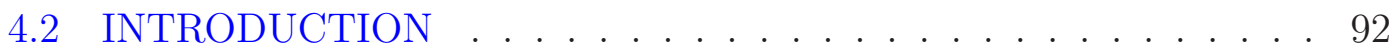

4.3 OBSERVATIONS . . . . . . . . . . . . . . . . . . . 95

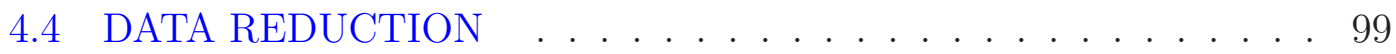

4.4.1 Spitzer/IRS Image Reduction . . . . . . . . . . . . . 99

4.4.2 Spectral Extractions . . . . . . . . . . . . . . . 100

4.4.3 Spectral Decomposition . . . . . . . . . . . . . 103

4.5 ANALYSIS . . . . . . . . . . . . . . . . . . . . . 108

4.5.1 Point Source Identification . . . . . . . . . . . . . 108

4.5.1.1 Young Star Clusters Exhibiting Silicates in Emission108

4.5.1.2 Silicate and PAH Emission Associated with a B[e] star ........................ 111

4.5.1.3 An Embedded Massive Young Stellar Object at the Edge of Hir Region N66A . . . . . . . . . . 112

4.5.2 Ionic Lines . . . . . . . . . . . . . . . . . . . . . . . . 115

4.5.3 PAH Feature Profiles . . . . . . . . . . . . . . . . 116

4.5.4 $14 \mu \mathrm{m}$ Continuum Emission . . . . . . . . . . . . . . 123

4.5.5 PAH Ratios as Diagnostics . . . . . . . . . . . . . . . . 124

4.5.6 N66 PAH Emission in Context . . . . . . . . . . . . . . . 130

4.5.7 Fraction of PAH Emission Coming From the Point Sources . 132

4.6 CONCLUSION . . . . . . . . . . . . . . . . . . . . . 134 
4.7 The Atlas of Reduced Spectra . . . . . . . . . . . . . . . . 136

5 Investigating Hydrogen Recombination Theory in HII Regions $\begin{array}{lr}\text { Around Starbursts } & 147\end{array}$

5.1 ABSTRACT . . . . . . . . . . . . . . . . . . . . 147

5.2 INTRODUCTION: Menzel's Cases and Modern Models . . . . . . . 148

5.3 The CLOUDY Code's Model Hydrogen Atom . . . . . . . . . . . . 152

5.4 Models with High-Resolution Input Spectra . . . . . . . . . . . . 153

5.5 The Use of Low-Resolution or Blackbody Spectra with CLOUDY . 158

5.6 Conclusions . . . . . . . . . . . . . . . . . 166

6 Conclusions and Future Directions 169

6.1 Conclusions . . . . . . . . . . . . . . . . . . . . . . 169

6.2 Future Directions . . . . . . . . . . . . . . . . . . . . . 174

A The Lucy Method of Computing Radiative Equilibria with Monte $\begin{array}{lr}\text { Carlo Techniques } & 176\end{array}$

A.1 INTRODUCTION . . . . . . . . . . . . . . . . . 176

A.2 The Lucy Method . . . . . . . . . . . . . . . . . . . . . . 177

A.2.1 Overview . . . . . . . . . . . . . . . 177

A.2.2 Initialization . . . . . . . . . . . . . . . . . . 178

A.2.3 Trajectories . . . . . . . . . . . . . . . . . . . . . 179

A.2.4 Monte Carlo Estimators . . . . . . . . . . . . . . 180

A.2.5 Temperature corrections . . . . . . . . . . . . . . . 182

A.3 Important Departures from the Lucy Method . . . . . . . . . . . . 183

B The Fractal Dust Distribution of a Super Star Cluster Embedding $\begin{array}{lr}\text { Envelope } & 186\end{array}$

B.1 Introduction . . . . . . . . . . . . . . . . . . . 186

B.2 Fractal Dust Distributions . . . . . . . . . . . . . . . . . 187

C Molecular Line Radiative Transfer with RATRAN 191

C.1 INTRODUCTION . . . . . . . . . . . . . . . . . . . . . . . . . 191

C.2 Radiative Transfer and Molecular Excitation Coupling . . . . . . . 192

C.3 Finding $J_{\nu}$ using the $\Lambda$-operator . . . . . . . . . . . . . . . . . . . 194

C.4 Outline of Code . . . . . . . . . . . . . . . . . . 196

$\begin{array}{lr}\text { Bibliography } & 199\end{array}$ 


\title{
Chapter 1
}

\section{An Introduction to Super Star}

\author{
Clusters and Applications of
}

\section{Conservation Laws Concerning}

\section{their Formation}

1.1 Observations of Super Star Cluster Environments

Super star clusters (SSCs) are massive young star clusters with high stellar densi-

ties $\left(\gtrsim 10^{3}\right.$ stars $\left.\mathrm{pc}^{-3}\right)$ and extreme pressures, most often found in merging galaxy 
systems, galactic nuclei, and blue compact dwarf galaxies [219, and references therein]. One of the closest such examples, R136 at the center of the 30 Doradus HiI region in the Large Magellanic Cloud (LMC), was at first believed to be an extraordinarily massive star - $2000 \mathrm{M}_{\odot}$ - due to its known luminosity, distance, and the poor resolution available at the time. This hypothesis directly challenged theoretical limits on stellar size: the Eddington standard model shows that radiation pressure plays the major role in stars greater than $\mathrm{M}_{*}=50 \mathrm{M}_{\odot}$, and that the star cannot remain bound past about $150 \mathrm{M}_{\odot}[68, \S 7.2 .7]$. It was Moffat [135] who first suggested that R136, like the central star cluster in the Galactic HiI region NGC 3603, might be a compact cluster of much more realistically massive young stars. Moffat ends his analysis with the leading question: "Are we witnessing a very young globular cluster being formed?"

SSCs were first discovered at optical wavelengths more than a decade earlier than Moffat's prediction. Shahbazian [183] recorded colors of bright starbursts in the arms and overlap regions of twelve nearby galaxies, and van den Bergh [11] studied their nuclear counterparts in M82. Arp \& Sandage [3] considered their observable properties and suggested that (what we now know as) SSCs in the galaxy NGC 1569 would be $10^{4} \mathrm{M}_{\odot}$ stars. Very little else was published on these pointlike starbursts until modern telescopes, such as the Hubble Space Telescope (HST), provided higher spatial resolution data to resolve SSCs from their surroundings. Holtzman et al. [79] used the Planetary Camera on the HST to study the stellar populations associated with compact, massive blue star clusters in NGC 1275. 
Conti \& Vacca [37] recognized that the starburst at the center of the blue compact dwarf galaxy (BCD) Henize 2-10 likely contained young massive clusters, and posited that they were globular cluster progenitors. O'Connell et al. [141] imaged extragalactic star clusters and found that they had properties very similar to R136, which was by this point known to be a cluster of stars [e.g. 89, 129]. O'Connell et al. [142] also studied a large population of stellar clusters in M82, and was one of the first to measure that these distant systems were very small: $\sim 3.5$ pc. Half-light radii of about $1.7 \mathrm{pc}[127]$ were discovered, as well as masses of about $10^{5-6} \mathrm{M}_{\odot}[199]$.

The effects of so many massive stars in close proximity were investigated soon after their first positing. Chevalier \& Clegg [30] proposed an analytical solution for a mechanical wind driven by a SSC of uniform mass and energy deposition. Their derived gas pressure could be directly compared to measurements in M82, with wind velocities derived from radio measurements, and is the primary explanation for understanding the phenomenon of supergalactic winds. More recently, this analytical approach has been extended $[186,187,201]$ in an attempt to understand how the wind cools (either adiabatically or radiatively), how energy deposition affects the surroundings, and whether supercritical, catastrophic cooling may act to inhibit winds.

Despite their enormous masses and densities, SSCs must still be born out of giant molecular clouds (GMCs), and therefore their earliest stages of formation are likely to be obscured from view at optical wavelengths. These so-called "embedded 
SSCs" were first detected in the radio $[108,210]$ in low-metallicity blue compact dwarf galaxies (BCDs) as compact sources with high ionizing fluxes. More were also found in the infrared, in starbursts like Arp 220 [184] and again in BCDs [175]. To date, the largest collected sample of embedded star cluster environments, extending from large OB associations to SSCs, has been publised in Aversa et al. [5], and provides a large atlas of radio continuum observations taken with radio telescope arrays.

Radio observations have confirmed the masses of embedded SSCs to be similar to those of more-evolved, optically visible SSCs. Furthermore, calculated high ionizing fluxes are equivalent to hundreds or thousands of O-stars and electron densities are $\sim 10^{3-4} \mathrm{~cm}^{-3}[9,100,101]$. These densities are similar to Galactic HII regions, suggesting heating from stars in a starburst environment, as opposed to active galactic nuclei (AGN), which may also be obscured but have far greater gas densities (e.g. $\left.10^{8-11} \mathrm{~cm}^{-3}\right)$.

The pressures associated with embedded SSCs are very high. Johnson \& Kobulnicky [101] determined $\mathrm{P} / \mathrm{k}_{B} \sim 10^{7-10} \mathrm{~cm}^{-3} \mathrm{~K}$. For comparison, in panspectral models of HiI regions, [46] considered pressures in the range $10^{4-8} \mathrm{~cm}^{-3} \mathrm{~K}$, in accordance with the HiI bubble theory driven by mass loss of [217] and formulated in [143]. [45, 47] determined that these radiation-driven HiI region pressures would lead to gas density gradients.

The most recent radio data on these objects suggest that the thick envelopes 
are porous, allowing a significant fraction of UV light to leak from the system $[102,159]$. The porous nature of embedded SSC environments is supported by UV and near-IR data of molecular hydrogen in low-metallicity blue compact dwarf galaxies (BCDs), which shows that the molecular gas only lies in clumps, not in the diffuse medium [205].

Whitmore [219] has shown that the youngest optically visible SSCs are about 2.5 Myr old. Whether this is due to clearing by supernovae from very massive progenitor stars or whether this is a stochastic effect from porosity remains to be seen.

With all of these observations under consideration, I will now focus on specific insights gleaned form detailed studies of SSCs, both optically visible and embedded, in the special environment of SBS 0335-052. This well-studied system shows not only how complicated gaining insight on SSCs can be, but also highlights a number of conundrums that have come to light concerning not only massive star formation but also star formation in extreme environments.

\subsubsection{The Special Case of SBS 0335-052}

SBS 0335-052 was determined by Izotov et al. [92] to have an oxygen abundance about $70 \%$ less than that of I Zw 18, the previous record-holder for lowest metallicity among galaxies. Its oxygen abundance varies between $7.11 \lesssim 12+\log _{10}(\mathrm{O} / \mathrm{H}) \lesssim 7.32$ in the bright, starbursting eastern portion of the galaxy (SBS 0335-052E) and 
as low as 6.86 in an HII region in the western part [95]. Not surprising, such a relatively pristine system is often the object of primordial helium abundance measurements, which are used to constrain Big Bang cosmological models [e.g. $94,134,144,152]$.

Long-slit optical spectroscopy has been used to study the star formation properties of the eastern portion of the galaxy, SBS 0335-052(E), as well as the more quiescent western portion (W). Izotov et al. [93] observed SBS 0335-052 to an unprecedented sensitivity. They found numerous high-ionization lines, including $\mathrm{Fe}^{4+}-\mathrm{Fe}^{6+}$, HeII $\lambda 4686$ emission over a wide extent, and $\mathrm{H}-\alpha$ emission over 68 kpc. These results suggested the enormous ionizing radiation levels that have been confirmed since at other wavelengths [e.g. 82, 159].

What is particularly interesting about SBS 0335-052(E) is that it hosts an embedded SSC in addition to those that are optically visible - and the dust mass is substantial. Hunt et al. [86] determined an $\mathrm{A}_{V} \sim 15$, Plante \& Sauvage [156] derived an even higher $A_{V} \sim 30$ based on the 6-100 $\mu \mathrm{m}$ SED, and further determined that optical and near-infrared stellar light could not be seen coming from the $2 \times 10^{6} \mathrm{M}_{\odot} \mathrm{SSC}$. The dust mass appears to be about $10^{5} \mathrm{M}_{\odot}$, and the grain size distribution is similar to that seen around active galactic nuclei (AGN). This is not surprising because the ionizing flux is so high for the embedded SSCs $\left(\sim 5 \times 10^{52}\right.$ photons $\mathrm{sec}^{-1}$; Reines et al. [159]) that small grains are preferentially destroyed. Houck et al. [82] showed that the total infrared SED was truly extraordinary: it peaks at about $20 \mu \mathrm{m}$, so that there is very little actual cold dust 
$\left(\sim 6 \times 10^{3} \mathrm{M}_{\odot}\right)$.

To explain how the embedded clusters are still bound and embedded in spite of the strong radiation field, Thompson et al. [203] invoked the idea of delayed ionization feedback, in which thick stellar winds absorb the ionizing photons in relatively close proximity to the most massive stars (within $\sim 1000 \mathrm{AU}$ ), quenching the ionizing flux. Delayed ionization feedback will delay the negative feedback that is necessary to push apart the embedding cloud, thereby prolonging the embedded phase in spite of strong winds from the massive stellar population. The explanation of delayed ionization feedback in terms of excess hydrogen line emission from collisions in the wind and ionization by Balmer photons was first given in Simon et al. [190].

Pustilnik et al. [158] first determined from $21 \mathrm{~cm}$ Hi emission that the East and West components were dynamically linked, and that the galaxy was dark matterdominated. Due to the presence of Hi tidal tail features, they also suggested that either mutual gravitational interaction, or else the presence of nearby spiral galaxy NGC 1376, was probably the triggering mechanism for this system's burst of star formation. There is also a clear age gradient observed across the system [53]. Thuan et al. [205] made the intriguing discovery that the Hi envelope surrounding SBS 0335-052 is not pristine, but appears to have been enriched at some point after the first stars of the system formed. This is prescient because it provides the only clue as to how such a low-metallicity system, and how other 
low-metallicity systems, can have so much dust associated with their starbursts: if infalling intergalactic gas is enriched, it provides the dust's raw material.

Thuan et al. [205] also determined that the $\mathrm{H}_{2}$ in SBS 0335-052 is not found in the diffuse medium, but only in clumps that are self-shielded from the hard UV radiation field so that molecular hydrogen can form on the available dust grains. The effects of clumpy gas distributions on observations was also discussed in Reines et al. [159] \& Johnson et al. [102]. Reines et al. found that the thermal radio emission was lower than expected compared to the optically-derived ionizing luminosity, suggesting that a substantial fraction of the UV light was leaking from the embedded systems. Johnson et al. [102] quantified this discrepancy, showing that $50 \%$ of the thermal radio emission was diffuse, once again suggesting light leakage (or a very strong radiation field from a diffuse stellar population as yet unobserved). Chapter 2 of this thesis shows that fractally distributed dust in embedded SSC envelopes can properly account for this observed phenomenon [218]. Likely explanations for this clumping include supersonic turbulence as has been discovered in giant molecular clouds [e.g. 107] as well as infalling material that forms streams [e.g. 72].

In summary, SBS 0335-052(E) offers a compelling picture for SSC formation that supplies a wealth of information about how massive star clusters are formed. In the absence of metals, the gas is still able to cool sufficiently to form stars. Gravitational instability across a region due to a distant gravitational partner can very likely act as a trigger. The source of gas for massive star cluster events in low-mass 
galaxies like BCDs can conceivably be funneled in from the intergalactic medium (IGM) and need not originate from the host galaxy; funneled IGM is one possible explanation for why the dust mass is relatively high compared to the gas-phase metals abundances. And lastly, clumpy dust and gas distributions occur naturally in these extreme star formation environments, and gas turbulence and gas infall provide natural explanations of the observed phenomena.

\subsubsection{What Questions Have Observations Left Unanswered?}

Despite the recent advances in understanding embedded SSCs as starburst events, our knowledge of SSC formation is largely heuristic, cursory, and, like star formation prescriptions generally, relies only lightly on fundamental physics. Revealing our dearth of understanding, SSC geometry and evolution are still poorly understood. For instance, the size of the giant molecular clouds that SSCs are born in, both mass and physical scale, are unknown because current instrumentation is not available to resolve these compact objects at long wavelengths. Radio and infrared wavelengths are required to probe the embedded phase of SSC evolution, because the cluster's young and hot stars are embedded in their natal giant molecular cloud. Radiated energy from an SSC is re-processed by the dust in the GMC and the electrons in the HII region as thermal infrared and free-free radio emission. Limits to the size of the central HiI region have been found by Johnson et al. $[99,100]$, Kobulnicky \& Johnson [108] of less than $\sim 7$ pc, which is approximately the same size scale as the canonical SSC half-light radius mentioned above. The 
full extent of an SSC's natal cloud, however, together with its density, including possible gradients, and mass, are still glaring unconstrained variables.

The questions of physical scale and mass of the embedding GMC are vitally important because they will likely shed light on issues that are contentious and ill-understood within the community. For instance, typical Galactic GMCs only collapse and convert $\sim 5 \%$ of their mass into stars [138]. If this is true for SSCs as well, then the initial GMC mass that is expected to create a $10^{6} \mathrm{M}_{\odot} \mathrm{SSC}$ must be $\sim 10^{7} \mathrm{M}_{\odot}$; observations of such large molecular clouds is quite rare. On the other hand, SSC-forming GMCs may undergo large-scale and high-percentage collapses, a supposition that is qualitatively backed by the known environments of these objects, where high pressures are known to be at play. And, as we shall see, simple calculations using energy and momentum conservation put realistic upper and lower limits on the gas conversion.

Just as importantly, the timescales of GMC dispersal are unknown. This is in part the subject of the next section, so I will only mention here that very young SSCs of $\sim 2.5 \mathrm{Myr}$ old have been observed in the optical in the overlap region of the Antennae galaxies [219].

The following section is an analytical look at the collapse and dispersal of GMCs associated with SSC formation. In it, I show that relatively simple calculations can be employed that bracket the likely known values of the percentage of gas that is converted into stars based on the energy released by the stars that are 
formed and assuming that the cluster must survive to be optically visible. This last assumption, based on the very high "mortality rate" of these objects [29], is another contentious point that will be discussed in more detail in $\S 1.2 .1$. I will then conclude with an outline of the work I have done to further our knowledge of these extreme star-forming environments.

\subsection{The Early Evolution of a Massive Star Clus- ter: Expectations from Simple Analytical Ar- guments}

Presumably, (but not yet observed), a SSC must be predated by a giant molecular cloud (GMC). When a cloud collapses and becomes gravitationally bound, and therefore more highly pressurized than its surroundings, the expectation is that it will form stars according to an initial mass function of some kind [e.g. 110, 171], including numerous massive stars. This expectation is confirmed by the numerous radio observations discussed above in which an optically invisible compact source is producing large numbers of ionizing photons deep inside its obscuring cloud.

Because SSCs are believed to be globular cluster progenitors as a class, we expect at least a subset of SSCs to be able to survive for several billions of years, excluding external effects. Therefore the evolution from GMC to SSC, and eventually to 
globular cluster, requires several steps, each of which can only be met under certain conditions.

\subsubsection{Cloud Dispersal}

Observational constraints suggest that SSCs become optically visible by about 3 Myr old [219]. The two known possible mechanisms for dispersing the molecular cloud are mechanical winds from $\mathrm{O}$ stars and Wolf-Rayet stars, or else the first supernovae. The first supernovae (the maximum mass of $\sim 100-120 \mathrm{M}_{\odot}$ is generally assumed based on observed massive stars; e.g. R136, Massey \& Hunter [129]) are also expected to go off at about 3 Myr old, but winds from stars begin at 0 Myr old and continue to be strong through the Wolf-Rayet phase, past $\sim 6 \mathrm{Myr}$ [e.g. 120]. We therefore have a small problem: the mechanism for dispersal is indeterminant. In order to provide order-of-magnitude tools that can be used to find out what mechanism removes the embedding cloud, we must define a timescale over which removal takes place. Let us therefore consider a GMC of $10^{6} \mathrm{M}_{\odot}$ with a radius of $25 \mathrm{pc}$, out of which a SSC might conceivably form. The free-fall time of such a GMC is:

$$
t_{f f}=\left(\frac{3 \pi}{32 G \rho_{0}}\right)^{1 / 2} \sim 2 M y r
$$


The free-fall timescale is, modulo some factors, almost the same as the system's crossing time $\left(\mathrm{t}_{c r} \sim 2.1 \times 10^{7}\left(\mathrm{r}_{h}^{3} / \mathrm{M}\right)^{1 / 2}=2.6 \mathrm{Myr}\right)$, and offers a rough divider between definitions of "fast" and "slow" cloud dispersal times: in the following analysis, anything that is significantly faster than the timescale of $\sim 2-3 \mathrm{Myr}$ will be considered "fast," whereas anything that is comparable to $~ 2-3 \mathrm{Myr}$ or longer will be considered "slow."

The removal of gas from a SSC environment by supernovae is fast. For an adiabatic blast wave with $10^{51-52} \mathrm{erg}$ of energy, we expect the gas to be removed from around a SSC in as little as $10^{4}$ years, and no greater than $5 \times 10^{5}$ years as an extreme outer limit $\left(\mathrm{r}_{\text {shell }}=12.4 \mathrm{pc} \times\left(\mathrm{E}_{S N} / 10^{51} \mathrm{erg}\right)^{0.2}\left(\mathrm{n}_{G M C} / 1 \mathrm{~cm}^{-3}\right)^{-0.2}\left(\mathrm{t} / 10^{4} \mathrm{yrs}\right)^{0.4}\right)$. The removal of gas from a SSC environment by stellar winds is, by contrast, slow. They begin to have an immediate effect on the cloud as soon as the stars are born and only substantially weaken after about 6 Myr old [120].

We now discuss the two limits of gas removal from an SSC environment, first discussing "fast" GMC dispersal that takes place on timescales much less than the free-fall timescale, and then discussing "slow" GMC dispersal that takes places on timescales similar to or slightly longer than the free-fall timescale.

\subsubsection{Fast GMC Dispersal}

If the GMC dispersal is fast, then energy balance should be able to help us determine the limits in which the SSC remains bound. Excluding pressure and 
magnetic fields, energy balance says that the total energy is the sum of the kinetic and potential energies [196]:

$$
E=K+\Omega
$$

for a solid body at constant density; $E$ is the total energy, $K$ is the system's kinetic energy, and $\Omega$ is the system's potential energy. In addition to energy balance, we expect the cloud to initially be in hydrostatic equilibrium, so the virial theorem should hold. The virial theorem states:

$$
\frac{1}{2} \frac{d^{2} I}{d t^{2}}=2 K+\Omega+3\left(\int P d V-P_{0} V\right)+\left(E_{m a g}-E_{m a g, 0}\right)
$$

where $I$ is the moment of inertia, $P$ is the pressure, $V$ is the volume, and $E_{m a g}$ is the magnetic energy. For simplicity, I am treating the cloud as a solid body as opposed to a collection of particles, so that $I, P$, and $V$ are idealized quantities. Additionally, for a first pass, I am assuming that pressure gradients, inertial accelerations, and magnetic fields are all negligible. These assumptions allow us to re-write the energy balance equation based on the fact that $2 \mathrm{~K}=-\Omega$ :

$$
E=\frac{\Omega}{2}=-K .
$$

For a mass loss of $\Delta \mathrm{M}$, the energy in the system changes by an amount: 


$$
E=E_{0}-E_{\Delta M} \propto(M-\Delta M)-(M-\Delta M)^{2},
$$

where the $(M-\Delta M)$ is proportional to the difference in kinetic energy (i.e. $\left.\mathrm{K} \propto \mathrm{mv}^{2}\right)$ and $(M-\Delta M)^{2}$ is proportional to the difference in the potential energy (i.e. $\Omega \propto M^{2} / R$ ). This expression can be put in terms of $\Delta M / M_{0}$ after substituting in the virial theorem:

$$
E=E_{0}-3 E_{0} \frac{\Delta M}{M_{0}}+2 E_{0}\left(\frac{\Delta M}{M_{0}}\right)^{2} .
$$

$\mathrm{E}_{0}$ is the initial energy of the system and is negative because we are assuming that we start with a bound system, and $\mathrm{M}_{0}$ is the initial mass of the system. This equation shows that if $\Delta \mathrm{M}$ is greater than $M_{0} / 2$, the total energy is positive and the cloud becomes unbound. Only values of $\Delta \mathrm{M}<0.5$ allow the total energy to remain negative and the cloud to remain bound.

Put another way, if more than $50 \%$ of the original system mass is removed, then the system will become unbound. This suggests that as a rough estimate, most of the original GMC mass must be converted into stars. However, the complete energy balance equation includes additional pressure and magnetic fields that may alter this value. There is some evidence that magnetics and turbulent pressure support play an important role in cloud support, possibly delaying star formation 
by lengthening the time that a GMC can survive prior to gravitational collapse $[42,136]$. We can express the energy balance as:

$$
E=K+\Omega+\left(\Pi-\Pi_{0}\right)+\left(E_{m a g}-E_{m a g, 0}\right)
$$

where $E_{\text {mag }}-E_{\text {mag }, 0}$ is the magnetic energy of the system minus that of the surroundings, and $\Pi-\Pi_{0}$ is the system's pressure minus the ambient pressure. In order to determine the effect of these extra terms on the system's energy balance, I assume that these extra terms function as an additive term in the energy balance, such that: $E=K+\Omega+E_{\text {extra }}$, and that the additional terms in the virial theorem also function as an additive term so that: $K=\frac{-\Omega+V_{\text {extra }}}{2}$ and $\Omega=-2 K+V_{\text {extra }}$. By including the extra energy and virial terms, $E_{\text {extra }}$ and $V_{\text {extra }}$, and with the knowledge that the additional terms are all directly proportional to the mass, then the total energy of the system scales with the new variable,

$$
\eta=V_{e x t r a, 0}+2 E_{e x t r a, 0}=\frac{1}{2} \frac{d^{2} I}{d t^{2}}-\left(\Pi-\Pi_{0}\right)+\left(E_{m a g}-E_{m a g, 0}\right) .
$$

The energy balance can then be re-written in terms of the initial energy, $E_{0}$, fraction of mass lost, $\Delta M / M_{0}$, and $\eta$ as:

$$
E=E_{0}-3 E_{0} \frac{\Delta M}{M_{0}}+2 E_{0}\left(\frac{\Delta M}{M_{0}}\right)^{2}+\eta \frac{\Delta M}{M_{0}}-\eta\left(\frac{\Delta M}{M_{0}}\right)^{2},
$$


or:

$$
E=E_{0}\left(1-\frac{\Delta M}{M_{0}}\right)\left(1-\frac{2 \Delta M}{M_{0}}\right)+\eta \frac{\Delta M}{M_{0}}\left(1-\frac{\Delta M}{M_{0}}\right)
$$

1- $\Delta \mathrm{M} / \mathrm{M}_{0}$ is the star formation efficiency (SFE), or the mass of the stars divided by the whole system mass. For SSCs, the SFE is thought to be on the order of 0.5 because not many GMCs are found above a few $\times 10^{6} \mathrm{M}_{\odot}$, whereas SSCs of about $10^{6} \mathrm{M}_{\odot}$ are relatively common. The effect of a non-zero value for $\eta$ can be visualized. In Figure 1.1, the system's energy normalized by the initial energy is plotted versus the change in the mass for a variety of $\eta$ values. The positive $\eta$ values suggest that the inertial and magnetic terms are the important ones. The negative $\eta$ values in turn suggest that the work done on the volume of gas is greatest. While nominal values of $\eta$ alter the $\Delta M$ value at which the system becomes unbound, this plot cannot show which term in $\eta, I$, $\Pi$, or $E_{\text {mag }}$, is most important over the timescale of cloud dispersal. Unfortunately, observations to date have not shed conclusive light on this issue. Some work finds that magnetic fields and ambipolar diffusion of the neutral material in a cloud cannot realistically be invoked to explain observations $[41,113]$, whereas other work finds that a significant, if not dominant, amount of the energy in a GMC is in magnetic fields [208]. What is clear is that both turbulence and magnetism do play a role in star formation, and that therefore non-negligible $\eta$ values should be expected.

What is interesting to note about Figure 1.1 is that even significant $\eta$ values of 


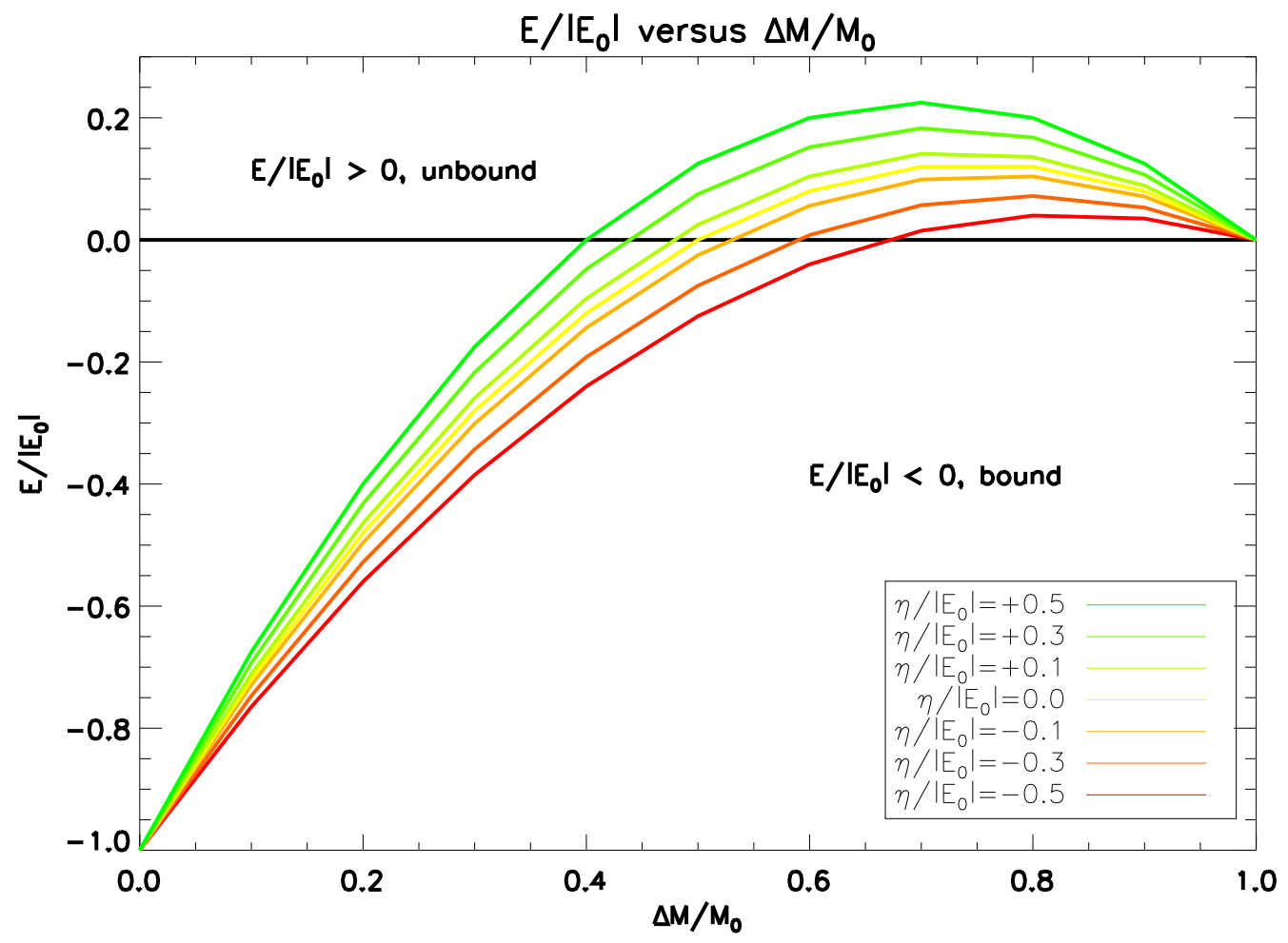

FiguRE 1.1: $\mathrm{E} /\left\|E_{0}\right\|$ versus $\Delta \mathrm{M} / \mathrm{M}_{0}$ for a variety of $\eta$ values.

\pm 0.5 do not drastically change the $\Delta \mathrm{M} / \mathrm{M}_{0}$ values for which the cloud remains bound. At the low end, $\Delta \mathrm{M} / \mathrm{M}_{0}$ is 0.5 for $\eta=+0.5$, whereas $\Delta \mathrm{M} / \mathrm{M}_{0}$ is 0.7 for $\eta=-0.5$. In this simple analytical model, even large contributions to the system's energy in magnetic field or turbulence have little effect on the amount of mass that would have to be lost before the system became unbound (less than 50\%).

Another way of looking at the effect of $\eta$ is shown in Figure 1.2. $\eta$ is plotted versus $\Delta M / M_{0}$ and the solid line is for a total system energy of zero; the bound and unbound regimes lie on either side of this line. This shows the realistic constraints on $\eta$ for the gamut of possible $\Delta M$ values for which $\eta$ is less than the absolute value of the cluster's original total energy. 


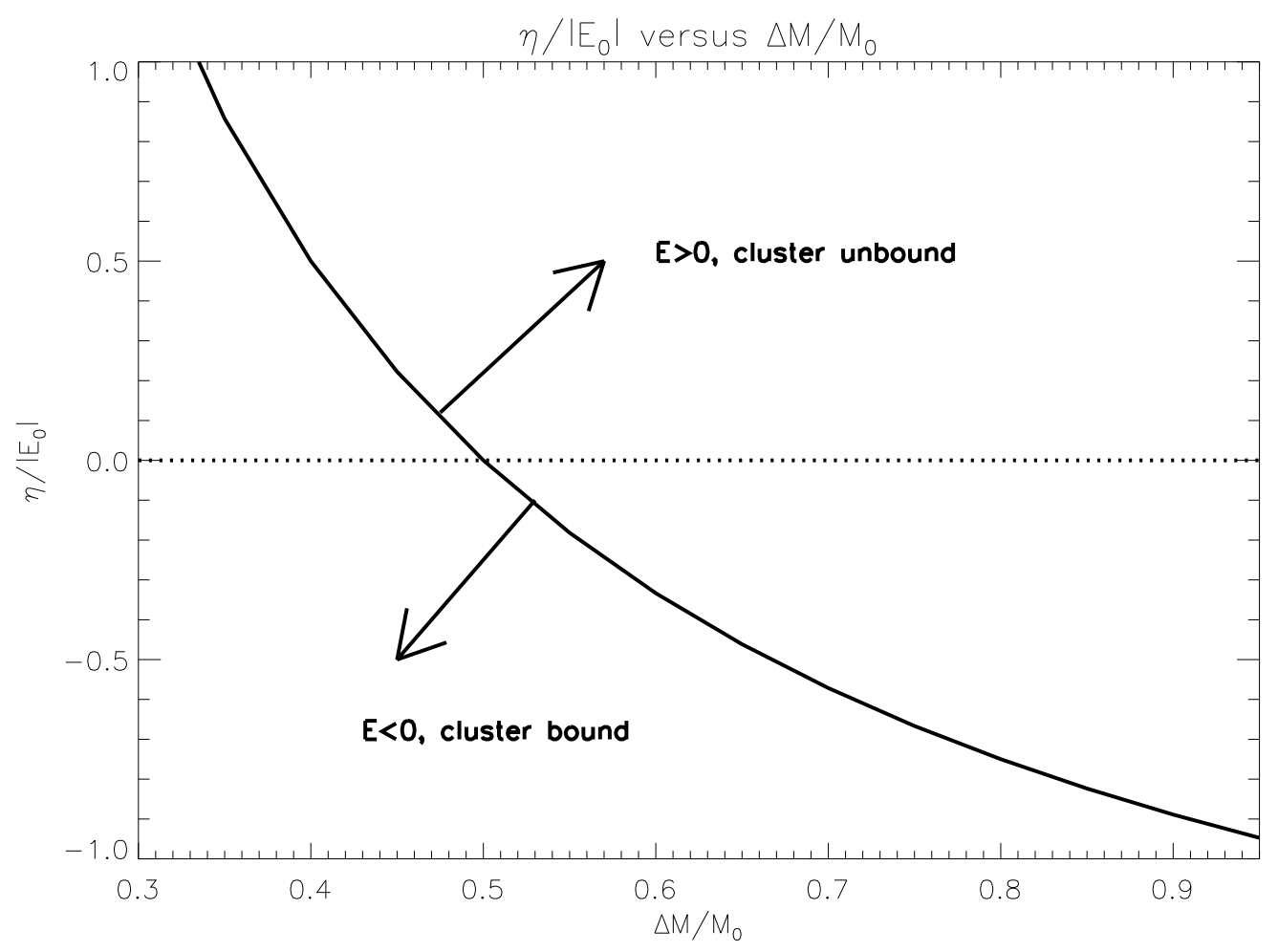

Figure 1.2: $\eta$ is plotted versus $\Delta \mathrm{M} / \mathrm{M}_{0}$ and the realistic regimes for unbound and bound clusters are shown. A dotted line shows the zero value for $\eta$, which bisects the $E=0$ curve at $\Delta M / M_{0}=0.5$ as shown in Figure 1.1.

While departures from the simplest prescription of the virial theorem, $2 K=-\Omega$, can be accounted for by the additional term $\eta$, it is very difficult to solve for $\eta$ analytically because there are so many forces acting upon the cloud. The rate of change in the moment of inertia depends on a number of factors: the mechanical power of the supernova wind; the wind's efficiency for interacting with the gas which is dependent on clumping, which is an unknown; and the Mach number of the gas, which can only be guessed at given our current understanding of the embedded phase of SSCs. The volume-integral of the cloud, and the ambient pressure, $\mathrm{P}_{0}$, are equally imponderable, though various stabs have been made at identifying realistic values both in the ISM and in SSC-forming GMCs [101, 133]. 
Lastly, there is very little available information on the magnetic fields associated with SSC environments. Even though these variables are all unconstrained, the virial theorem is able to show us that values $0.4<\Delta M / M_{0}<0.7$ are reasonable first guesses for the amount of material lost by the system in the limit of fast cloud dispersal.

\subsubsection{For Slow GMC Dispersal}

In the limit that the GMC is not dispersed by the first supernovae but by the mechanical luminosity in stellar winds, then the limit of slow mass removal means that $\eta=\frac{d^{2} I}{d t^{2}}-\left(\int P d V-P_{0} V\right)+\left(E_{m a g}-E_{m a g, 0}\right)$ value can be set to zero and the virial theorem will be simply: $2 K=-\Omega$. In this limit, we can take the derivative of the energy balance with respect to mass:

$$
\frac{d E}{d M} \propto \frac{K}{M}-\frac{2 \Omega}{M}=\frac{3 E}{M}
$$

We integrate:

$$
\int_{E_{0}}^{E} \frac{d E}{E}=\int_{M_{0}}^{M} \frac{3 d M}{M}
$$

which yields 


$$
\frac{E}{E_{0}}=\left(\frac{M}{M_{0}}\right)^{3}
$$

and since $\mathrm{E} \propto \Omega \propto \frac{M^{2}}{R}$, we can re-write this as:

$$
\frac{M}{M_{0}}=\frac{R_{0}}{R}
$$

For very slow cloud dispersal, the cloud will expand such that $M R$ is constant. This will be more realistic than the quick dispersal case if only the stellar winds contribute to the dispersal of the GMC.

\subsubsection{Taking Both Stellar Winds and Supernovae Into Ac- count}

In reality, we should expect the GMC to disperse in a manner that is somewhere between these two extremes. During the embedded phase, the O star winds do not quickly destroy their cloud as evidenced by the fact that these systems only become optically visible at about $3 \mathrm{Myr}$. This may be because each $\mathrm{O}$ star is embedded in an ultra-compact HII region [UCHII; 224] which delays photoionization feedback, as posited by Thompson et al. [203]. However, by the time supernovae begin to go off, there may already be a substantial and unified HII region present [this was assumed in 218]. Since the stellar wind from massive stars drops off precipitously 
[120] as the O- and B-type stars age and die off, it is sensible to imagine that the stellar winds begin the GMC dispersal, which is then finished off by the first supernovae.

Yao et al. [231] considered the dynamics of the dispersal of the GMC from the growth of the common HII region cavity until the SNe go off. Starting with the HII expansion strictly from ionization, which Draine [49] estimates will take only a few thousand years, the radius of the HiI region goes as [195]:

$$
R_{H I I}(t)=R_{S}\left(1+\frac{7}{4} \frac{c_{i} t}{R_{S}}\right)^{4 / 7}
$$

where $R_{S}$ is the initial size of the Strömgren sphere and $c_{i}$ is the speed of sound in the ionized gas. I note especially that this theory may not hold for SSCs if the individual massive $\mathrm{O}$ stars are individually embedded in UCHIIs that delay photoionization feedback. However, when the stellar winds dominate the HiI region expansion, the radius continues to grow at a rate that is weakly dependent on the mechanical luminosity of the stellar wind [132]:

$$
R_{w}(t)=269.0\left(\frac{L_{38}}{n}\right)^{1 / 5} t_{7}^{-2 / 5}
$$

where $L_{38}=L_{w} /\left(10^{38} \mathrm{erg} / \mathrm{s}\right)$ and $t_{7}=t /\left(10^{7} \mathrm{yr}\right)$. The expansion due to the wind may be a short-lived stage, according to Yao et al. [231]; since a $120 \mathrm{M}_{\odot}$ star may go supernova as early as $t=7.0 \times 10^{5} \mathrm{yr}$ [125], the expansion due to the supernova 
ejecta is likely to take over fairly rapidly. Yao et al. assume an adiabatic expansion of the supernova ejecta, for which the radius will expand with time as:

$$
R_{S N}(t)=97.0\left(\frac{N_{E_{51}}}{n}\right)^{1 / 5}\left[t_{7}^{3 / 5}-\left(\frac{t_{1 s t S N}}{10^{7}}\right)^{3 / 5}\right]+R_{w}\left(t_{1 s t S N}\right)
$$

This phase continues until radiative energy losses become large enough for adiabatic assumptions to be no longer tenable. During this so-called "snowplow phase," [132], the radius of the HiI region continues to expand as $t^{1 / 4}$.

Draine [48] developed a body of static equilibrium HiI region models that took radiation pressure and dust into account. He found that the central ionized gas densities are substantially smaller than those near the ionized boundary. This results in a larger fraction of the gas being ionized than in traditional uniformdensity models. He also calculated that dust drift through the ionized plasma, which may be important after the Strömgren sphere has expanded to its full size because it allows dust grains to drift into the neutral material, is slower than the speed of the expanding ionization front and therefore an unimportant factor in HiI region dynamics. When applying these theories to observations of the lowdensity bubble inside the HII region N49, Draine [48] determined that the central cavity was already too strongly peaked than could be explained by photoionization alone; the $\mathrm{O} 6.5 \mathrm{~V}$ star at the center of the region is likely already producing a dynamically-important stellar wind that is aiding with HII region expansion. 


\subsubsection{Truly Realistic Models Treatment}

The biggest hindrance to the usefulness of the above analytical study of fast/slow cloud dispersal is that it assumes that the GMC is in complete isolation. Using the powerful GLIMPSE survey of the Galactic Plane [10, 35], numerous examples of PAH-bright filaments from the diffuse ISM towards star-forming regions have been found and modeled [34, 71]. The conclusion of these studies is that accretion from the ISM increases the cloud's mass by roughly a factor of two. SSCs likely form under similar circumstances of ISM accretion, so that the meaning of the term "star-formation efficiency" should be called into question: do we mean the efficiency from the original GMC mass, or the eventual system mass, or the mass at the time that the majority of the stars were formed? A truly realistic treatment of SSC early evolution would have to take into account the increase in system mass prior to and during feedback from the supernovae and stellar winds. Such a treatment would mean that, at the simplest, this is analytically a two free parameters problem: both the system mass and $\eta$ are unknowns.

Regardless of how the GMC becomes dispersed, an SSC eventually becomes optically visible, is surrounded by a large HiI region, a photodissociation region (PDR) at the outer edge of the HiI region, and fragmented remnants of the GMC; see Indebetouw et al. [91] for an in-depth analysis of the HiI region/PDR/GMC 30 Doradus around the SSC R136 with figures that illustrate this three-part morphology. The dynamical details of how an SSC environment is dispersed must begin with a 
detailed discussion of the known observational properties of SSC environments at their earliest embedded and HiI region phases. This thesis deals with predictions and observations of early SSC evolution.

\subsection{The Direction of this Thesis}

Chapters 2-5 provide detailed studies of important observable features associated with a SSC's early evolutionary phases. Chapters 2 and 3 predict the dust and molecular gas emission characteristic of possible geometries of the GMC around embedded SSCs. Chapter 4 deals with gas and dust properties in a giant Hiı region in the Small Magellanic Cloud (SMC). Chapter 5 deals with Hydrogen recombination theory in HiI regions, where we consider the importance of emission line pumping by the stellar continuum on the emergent line fluxes. Below, I outline these chapters by way of introducing the subject matter.

Chapter 2 is a paper on numerical models of dust emission from embedded SSC environments that cover a large swathe of observable parameter space. The code used was the pre-cursor to Barbara Whitney's hochunk-3d, and required significant updating to make it run accurately. This body of models offer a direct comparison sample with which to study individual embedded SSCs after the advent of the next generation of space-based infrared observatory; i.e., the James Webb Space Telescope. This paper has been published in the Astrophysical Journal (2011, 729, 111). 
Chapter 3 is an extension of embedded SSC environment studies, to model the molecular emission. Comparisons between models and observations are now becoming possible with the Atacama Large Millimeter/submillimeter Array. This work was presented at American Astronomical Meeting 219 as poster presentation 341.06.

Chapter 4 is a study of one particular massive star cluster's giant Hiı region (N66 in the Small Magellanic Cloud), and makes a detailed study of the properties of the dust and gas properties during the HII region phase of massive star cluster evolution. The data for this project was obtained from the Spitzer Space Telescope, all of which I reduced and analyzed. This work has been published in the Astrophysical Journal (2013, 771, 16).

Chapter 5 takes a detailed look at Hydrogen recombination line theory in HII regions. We investigate the importance of line pumping by the ionizing source continuum, and conclude that its contribution to the lines can be as much as $15 \%$ in certain cases. However, pumping and subsequent fluorescence can appear to contribute much more to line fluxes if a low-resolution ionizing source spectrum or simple blackbody curve is used, as is often the case in the recent literature. For the most realistic geometries, line pumping is practically negligible, and Case B is the favored description of the state of the gas. This work has not yet been published.

In the Conclusions, I offer a road map for continued work in the area of massive star cluster formation and early evolution. The appendices offer some numerical 
Chapter 1. An Introduction to Super Star Clusters and Applications of Conservation Laws Concerning their Formation

background that will be useful to anyone wishing for a (relatively) elementary introduction to the computations that are at the heart of many of the modeling aspects of this thesis. 


\title{
Chapter 2
}

\section{The Infrared Properties of}

\section{Embedded Super Star Clusters:}

\author{
Predictions from
}

\section{Three-Dimensional Dust}

\section{Radiative Transfer Models}

\subsection{ABSTRACT}

With high-resolution infrared data becoming available that can probe the formation of high-mass stellar clusters for the first time, appropriate models that make 
testable predictions of these objects are necessary. We utilize a three-dimensional radiative transfer code, including a hierarchically clumped dusty envelope, to study the earliest stages of super star cluster evolution. We explore a range of parameter space in geometric sequences that mimic the hypothesized evolution of an embedded super star cluster. The inclusion of a hierarchically clumped medium can make the envelope porous, in accordance with previous models and supporting observational evidence. The infrared luminosity inferred from observations can differ by a factor of two from the true value in the clumpiest envelopes depending on the viewing angle. The infrared spectral energy distribution (SED) also varies with viewing angle for clumpy envelopes, creating a range in possible observable infrared colors and magnitudes, silicate feature depths and dust continua. General observable features of cluster evolution differ between envelopes that are relatively opaque or transparent to mid-infrared photons. For optically thick envelopes, evolution is marked by a gradual decline of the $9.8 \mu \mathrm{m}$ silicate absorption feature depth and a corresponding increase in the visual/ultraviolet flux. For the optically thin envelopes, clusters typically begin with a strong hot dust component and silicates in emission, and these features gradually fade until the mid-infrared PAH features are predominant. For the models with a smooth dust distribution, the Spitzer MIPS or Herschel PACS [70]-[160] color is a good probe of the stellar mass relative to the total mass, or star formation efficiency. Likewise, the IRAC/MIPS [3.6]-[24] color can be used to constrain the $\mathrm{R}_{\text {in }}$ and $\mathrm{R}_{\text {out }}$ values of the envelope. However, clumpiness confuses the general trends seen 
in the smooth dust distribution models, making it harder to determine a unique set of envelope properties. Nevertheless, good diagnostic colors were found for each of the input parameters: again, the [70]-[160] color can be used to separate models with different star formation efficiencies; the Spitzer IRAC/MIPS [8.0]-[24] color is able to constrain the inner and outer radii, $\mathrm{R}_{\text {in }}$ and $\mathrm{R}_{\text {out }}$, values; and the IRAC [3.6]-[5.8] color is sensitive to the fraction of the dust distributed in clumps. Finally, in a comparison of this model set to IRAS data of ultracompact Hir regions, we find good agreement, suggesting that these models are physically relevant, and will provide useful diagnostic ability for datasets of resolved, embedded SSCs with the advent of high-resolution infrared telescopes like James Webb Space Telescope.

\subsection{INTRODUCTION}

Super star clusters (SSCs) are massive young star clusters with high stellar den-

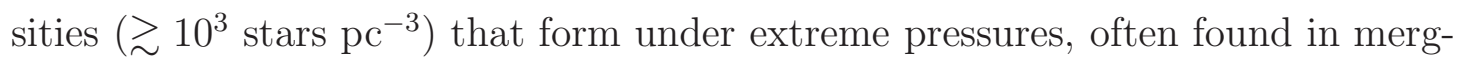
ing galaxy systems, galactic nuclei, and blue compact dwarf galaxies [219, and references therein]. With masses typically in excess of $10^{5} M_{\odot}$, they are the most massive type of stellar cluster known. Studies of the most nearby analogues Westerlund 1 [26] and R136 [16] show that these objects are consistent with expectations that they are globular cluster progenitors; this expectation is strengthened by numerous studies of SSC populations outside the Local Group [e.g. 37, 79, 97, 141, 179]. Because of their high density of massive stars, models 
show that SSCs have the ability to disperse metals from supernovae ejecta to large distances, trigger further star formation episodes, and act as a launching mechanism for super-galactic winds [139, 228]. Therefore SSCs can have a major impact on their host galaxies.

Tracking the early evolution of SSCs requires long wavelength observations due to the dust-enshrouding birth envelopes that surround them at young ages. Embedded SSCs were first detected in the radio [e.g. 108, 210] in low-metallicity blue compact dwarf galaxies (BCDs) as compact sources with high ionizing fluxes. Numerous other examples have also been found in the infrared, in starbursts such as Arp 220 [184] and again in BCDs [175].

Radio observations have confirmed the masses of embedded SSCs to be in the range found for their optical counterparts. Furthermore, the calculated high ionizing fluxes are equivalent to hundreds or thousands of O-stars and electron densities are $\sim 10^{3-4} \mathrm{~cm}^{-3}[9,100,101]$. These densities are similar to those found in Galactic HII regions, but are significantly lower than typical densities associated with Active Galactic Nucleus Broad Line Regions $\left(>10^{8} \mathrm{~cm}^{-3}\right)$ suggesting that SSCs are also heated by young stars in a starburst environment, and not AGN.

Despite advances made this last decade in understanding embedded SSCs as starburst events, their early evolution is still poorly understood. Properties such as the mass and physical size of the clouds from which they are formed and the amount 
of gas that is turned into stars [i.e. the star formation efficiency; 4] are not wellconstrained. In addition, the most recent radio, near-infrared, and ultraviolet data on these objects suggest that the thick envelopes are porous, allowing a significant fraction of UV light to leak from the system $[102,159,205]$. This project is a first attempt at modeling the infrared spectral energy distributions of embedded super star clusters. By investigating a large variation in the values of the input parameters in a hierarchically clumpy and porous envelope, these models can be used to constrain the dusty envelope geometry and star formation efficiency of embedded super star clusters.

The decision to model embedded SSCs in the infrared is based on the fact that they are most visible in the infrared and radio; at these young ages, hot stars heat the dust in the circum-cluster envelope and excite free-free radio emission inside the HiI region. Therefore these wavelengths are essential for studying the dust properties during the embedded phase, the dust mass, and the envelope geometry. The radiative transfer models presented in this work offer predictions about what we expect embedded SSCs to look like in the infrared and what wavelength observations offer the best diagnostic capability. With telescopes like JWST on the horizon, its high spatial resolution and mid-infrared sensitivity making it far better than existing infrared space facilities [194], we may finally be entering a period when questions about initial dust mass and geometry of a SSC's embedded phase can be answered on a global scale. In the near term, observations using Herschel and Spitzer may be used to probe unresolved embedded SSCs, and these 
Chapter 2. The Infrared Properties of Embedded Super Star Clusters: Predictions from Three-Dimensional Dust Radiative Transfer Models

models can be used to estimate their physical size and geometry.

In this paper we present simulated infrared images, SEDs, and colors of embedded SSCs along a geometric sequence that mimics the evolution of a young embedded super star cluster. We discuss the models in detail in $\S 2.3$, then present model images, SEDs and colors in $\S 2.4$. We compare unresolved populations to the models in $\S 2.5$, discuss the limitations of these models when comparing to resolved populations in $\S 2.6$, and conclude in $\S 2.7$.

\section{$2.3 \quad$ MODELS}

\subsubsection{The Radiative Transfer Method}

The models presented in this paper calculate radiative transfer from thermal dust grains and stochastic small grains, and scattering from the dust. The code simulates a three-dimensional geometry, and is based on previous work on thermal dust grains in two dimensions [see 27, 220], updated to include three dimensions and clumpy dust distributions [90], and stochastic emission from polycyclic aromatic hydrocarbons and very small grains [PAHs/VSGs; 225].

The code uses a Monte-Carlo technique that issues 'photon packets' into a dusty envelope from a central source. Packets that encounter large grains either change the temperature in the grid space in which the encounter occurs, according to 
the prescription of Lucy [123], or are scattered according to a modified HenyeyGreenstein function [39]. A small number of packets encounter PAHs/VSGs, and are re-emitted according to emissivity templates from Draine \& Li [51]; see Wood et al. [225] for details about emission from PAHs/VSGs in our models.

Embedded SSCs are believed to have porous envelopes that allow a significant portion of the ultraviolet (UV) light to escape from the system [supporting observational evidence is provided in $\S 2.3 .5$ from 102, 159, 205]. To account for this fact, we have included a hierarchically clumped density structure for the dusty envelope, using the prescription of Elmegreen [56].

In order to confine the parameter space investigated in this model set, we have varied only four input parameters that most influence the output spectral energy distribution: the inner and outer dust radii in a spherical geometry; the mass in stars divided by the total cluster mass [i.e. the 'star formation efficiency' or SFE; 4]; and the fraction of dust that is smoothly, as opposed to fractally, distributed. The many other parameters that remain fixed, such as the central star cluster mass, the IMF, the central source luminosity and age, the dust species used, the volume fractal dimension adopted, and the radial dust distribution exponent, are all described in $\S 2.3 .2$ to $\S 2.3 .4$. 


\subsubsection{The Dust Composition}

We use the standard dust model size distribution derived in Kim et al. [105] that fits an extinction curve with $\mathrm{R}_{V}=4$ using the prescription of Cardelli et al. [24]. The size distribution is not a simple power law, but is derived from a Maximum Entropy Method solution, which gives the best solution compatible with the extinction data. The chosen $\mathrm{R}_{V}$ value has been found to be appropriate for the more dense regions of molecular clouds [221] and therefore we make the assumption that it will also fit embedded SSCs.

We adopt the dielectric functions of astronomical silicate and graphite grains from Laor \& Draine [112]. Our grain model includes a layer of water ice on the grains, covering the outer $5 \%$ in radius. The ice mantle increases the opacity of the grains at all wavelengths, but the change is most pronounced longward of $35 \mu \mathrm{m}$. The slope of the opacity with wavelength is the same in the infrared for grains with and without the ice mantle, so any error introduced by the inclusion of an ice mantle is a scalar factor at the wavelengths of concern in this paper. The ice dielectric function is from Warren [216].

The PAHs/VSGs are templates from Draine \& Li [51]. The energy density of the radiation field relative to the interstellar radiation field (ISRF) can vary between $0.5 \leq U \leq 10^{7}$ and the ionization fraction is that used in Draine \& $\operatorname{Li}[51]$ to constrain the models to reproduce the observed Milky Way spectrum. Only grains that are $\leq 200 \AA$ in size are considered small grains. Grains larger than this are 
Chapter 2. The Infrared Properties of Embedded Super Star Clusters: Predictions from Three-Dimensional Dust Radiative Transfer Models

assumed to thermalize after photon interactions, and are therefore considered large grains. The PAH/VSG mass fraction in dust used in this paper is $3.55 \%$, which is the average value found for 65 galaxies in the SINGS survey [50]. This is therefore an acceptable value to expect for extragalactic embedded SSCs.

\subsubsection{The Central Source}

In these models, the central cluster is treated as a point source with the spectrum of a $10^{6} \mathrm{M}_{\odot}$ star cluster, produced by the Starburst99 spectral synthesis models [120]. The input metallicity is solar and the initial mass function is a Salpeter IMF [171]; changing these parameters was found to have little effect on the overall shape of the resulting infrared SED, validating the decision to hold them constant. The age is set to 1 Myr throughout the geometric sequence to ensure that the UV luminosity remains constant. Since evolution is expected to take place in less than three or four million years, prescribing a fixed age is warranted - see $\S 2.3 .5$ for a discussion about the evolutionary pace for embedded super star clusters. The instantaneous starburst mode was chosen, without continuum emission. The luminosity of the source is $1.6 \times 10^{9} \mathrm{~L}_{\odot}$.

\subsubsection{The Envelope Properties}

The dust and gas around the central source is distributed spherically with variable inner and outer radii and envelope mass. The initial envelope mass is set for star 
formation efficiencies (SFEs) of $5 \%, 10 \%, 15 \%, 25 \%$, and $50 \%$, which is a large range of possible values based on observations of star-forming regions [15, 138]. The dust can be distributed fractally and smoothly, and the percentage of dust that is smoothly distributed is varied from between $100 \%$ smooth to $1 \%$ smooth (i.e. $0 \%$ to $99 \%$ clumpy).

The models mimic a cluster's evolution by moving the inner radius out towards the outer radius in a geometric sequence; Table 1 lists the sequence for the three possible outer radii we have chosen, and Figure 2.1 shows how the geometric progression roughly follows what is expected for a real SSC. At very young ages, each star will realistically inhabit its own compact Hin region, but as the cluster evolves, these compact regions will grow and merge into a single, large cavity. Assuming a stationary stellar wind as in the semi-analytical and numerical models presented by Tenorio-Tagle et al. [202], this cavity will grow and disperse, eventually leaving the central SSC exposed. The initial inner radius of the models, correlated to intra-cluster dust, is $0.1 \mathrm{pc}$, and moves out to the outer radius, which remains fixed. 


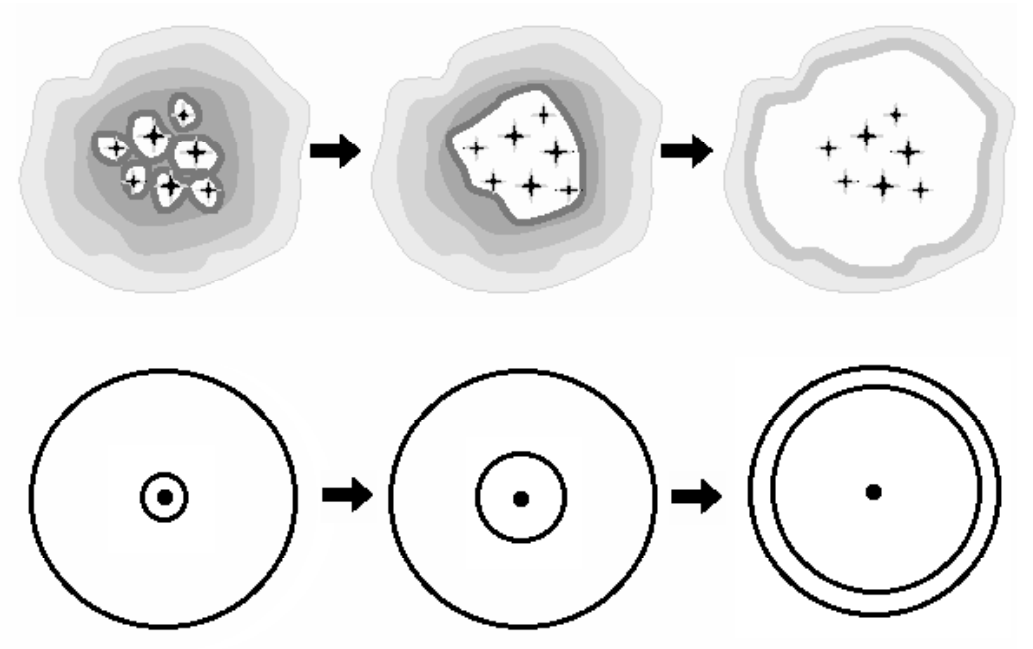

FIgURE 2.1: A cartoon illustrating the early stages of a super star cluster's evolution and our corresponding model estimates. The smallest inner radius presented in this paper, $R_{\text {in }}=0.1 \mathrm{pc}$, is likely to correspond to intra-cluster dust in a real cluster (left-most figure), which will only exist while the constituent stars still have individual cocoons.

Table $1 . \mathrm{R}_{\text {in }}$ values.

\begin{tabular}{ccc}
$\mathrm{R}_{\text {out }}=25 \mathrm{pc}$ & $\mathrm{R}_{\text {out }}=50 \mathrm{pc}$ & $\mathrm{R}_{\text {out }}=100 \mathrm{pc}$ \\
\hline $0.1 \mathrm{pc}$ & $0.1 \mathrm{pc}$ & $0.1 \mathrm{pc}$ \\
$0.5 \mathrm{pc}$ & $0.5 \mathrm{pc}$ & $1.0 \mathrm{pc}$ \\
$1.0 \mathrm{pc}$ & $1.0 \mathrm{pc}$ & $5.0 \mathrm{pc}$ \\
$2.0 \mathrm{pc}$ & $5.0 \mathrm{pc}$ & $10 \mathrm{pc}$ \\
$3.0 \mathrm{pc}$ & $10 \mathrm{pc}$ & $15 \mathrm{pc}$ \\
$6.0 \mathrm{pc}$ & $15 \mathrm{pc}$ & $25 \mathrm{pc}$ \\
$9.0 \mathrm{pc}$ & $20 \mathrm{pc}$ & $35 \mathrm{pc}$ \\
$12 \mathrm{pc}$ & $25 \mathrm{pc}$ & $45 \mathrm{pc}$ \\
$15 \mathrm{pc}$ & $30 \mathrm{pc}$ & $55 \mathrm{pc}$ \\
$18 \mathrm{pc}$ & $35 \mathrm{pc}$ & $65 \mathrm{pc}$ \\
$21 \mathrm{pc}$ & $40 \mathrm{pc}$ & $75 \mathrm{pc}$ \\
$24 \mathrm{pc}$ & $45 \mathrm{pc}$ & $95 \mathrm{pc}$ \\
\hline
\end{tabular}


The SFE values from $5 \%$ to $50 \%$ presume initial cocoon masses in the range $1.9 \times 10^{7} \mathrm{M}_{\odot}$ down to $1 \times 10^{6} \mathrm{M}_{\odot}$. For a given $\mathrm{SFE}$ value and outer radius, the average mass density is constant with radius, so that the mass of the shell in a geometric sequence scales with the inner and outer radii as: $M \propto R_{\text {out }}^{3}-R_{\text {in }}^{3}$. The average mass densities range from $10^{5} \mathrm{~cm}^{-3}$ at the densest to $10 \mathrm{~cm}^{-3}$ at the least dense, where the densest models are for a SFE of $5 \%$ and an outer radius of $25 \mathrm{pc}$ while the least dense models are for a SFE of $50 \%$ and an outer radius of 100 pc.

The envelope is assumed to have a standard dust-to-gas ratio of 0.01 . It is furthermore seeded with PAHs/VSGs to a depth equal to $A_{V}=1.5$ from the ionizing source. This is because the UV flux is sufficiently attenuated after this depth that we expect very little UV heating of small grains in the rest of the envelope. Comparisons between models run with PAHs/VSGs throughout the dusty envelopes and models PAHs/VSGs in to a depth of $A_{V}=1.5$ show that PAH/VSG emission changes only slightly for models where $A_{V} \lesssim 250$.

There are five clumpy dust fractions presented: 0, 0.1, 0.5, 0.9, \& 0.99 clumpy. The spread in clumpy fraction is the complete range of conceivable clumpy values that could be found. The volume fractal dimension was chosen as the best value from Elmegreen [56] which matched the turbulent intercloud medium, $\mathrm{D}=2.3$. The fractal length $\mathrm{L}$ is taken to be 3.792 so that the maximum density of the clumps can be computed per hierarchic level, $\rho(h)=L^{(3-D) h+H D}$ where $h$ is the hierarchical level in question and $H$ is the maximum hierarchical level, which we have set equal 
to five; higher $H$ values create fractal sizes smaller than the resolution elements in our three-dimensional grid.

\subsubsection{Supporting Observational Evidence for the Model Parameter Choices}

The model parameters were chosen to be consistent with observations of embedded SSCs, and the parameter space is corroborated with observational evidence from the literature in this section.

With regard to dust grains types, the PAH models from Draine \& Li [51] are the most suitable available to date. Ongoing research $[51,64]$ that accounts for different PAH ionization fractions and redder exciting sources than those used here would likely change individual PAH band fluxes. However, since we are not interested in $\mathrm{PAH}$ ratios in this work, the inclusion of these processes does not impact the results of this study.

The ice mantle on the dust grains is suitable for dense, cold regions, such as young star formation regions, where a large part of the dust is not being heated by the embedded stars and the water-ice cannot be sublimated from the grains. At any optical depth it has been shown that the water-ice features will be masked and therefore hard to detect [162]. Since there is observational evidence for water associated with compact, young star clusters [21] and the slope of the opacity 
functions with and without water ice are the same, we have left the dust with ice mantles throughout the entire geometric sequence.

Fixing the cluster age is based on upper limits of the time it takes for a cluster to emerge from its envelope. Observations with HST show optically visible clusters with ages of just a few Myr [e.g. 98]. Age estimates are fairly insensitive below about 2.5 Myr. Radio observations have also suggested the same approximate age, so we can assume a fiducial emergence timescale of about three Myr for a super star cluster [108, 219]. Since the UV continuum, which most affects the resulting infrared SED, does not significantly change between ages of 0 and 3-4 Myr, setting the cluster age to 1 Myr throughout the sequence is warranted in order to limit the number of free parameters.

The models are run under the assumption that the central star cluster is a point source and the envelope is perfectly spherical; this is a necessary simplification to confine the parameter space in this study. However, the high stellar densities and small half-light radii discovered for optically-visible SSCs [127, 199] suggest that the central source is fairly localized in its formation envelope. Furthermore, placing dust to within $0.1 \mathrm{pc}$ of the central point source will produce the high dust temperatures expected from intracluster dust in a real embedded SSC. Dust sublimation is not a concern even for these most embedded model phases: the dust sublimation radius, assuming a sublimation temperature of $1600 \mathrm{~K}$, is $0.03 \mathrm{pc}$, well within the smallest inner radius value adopted. 
The outer radii of 25, 50, and $100 \mathrm{pc}$ were chosen based on estimates from observational results. Vacca, Johnson, \& Conti [211] model infrared and radio observations of 'ultra-dense' HiI regions in Henize 2-10 as scaled-up versions of Galactic ultra-compact HiI regions to derive their radial size. Hunt et al. [87] based their estimates on models of global dwarf galaxy SEDs for which the radii of the actively star-forming regions are derived based on infrared luminosities and temperatures.

Lastly, in order to be consistent with the growing body of evidence that suggests that the ISM is clumpy in a large variety of environments, a clumpy envelope was included around the central star cluster. That the ISM is not uniform is well established [e.g. 57, 106, 213]. In star formation environments, recent work has begun to show that the same is true. Thuan et al. [205] shows that the molecular hydrogen in a BCD galaxy is clumpy because emission is visible in the near-IR, which is sensitive to dense $\mathrm{H}_{2}$, but not in the UV, which is sensitive to diffuse $\mathrm{H}_{2}$. Reines et al. [159] and Johnson et al. [102] show that for different clusters, more than $40 \%$ and $50 \%$ of the UV flux is detected outside the embedded sources. This could either be because the ISRF is very strong around the clusters or because there is light leaking from the clusters. Most recently, model-dependent results by Eldridge \& Relaño [54] shows that about $50 \%$ of the ionizing flux is leaving the giant HII region NGC604, though this latter result is highly dependent on ages derived from observations of Wolf-Rayet stars. 
Chapter 2. The Infrared Properties of Embedded Super Star Clusters: Predictions from Three-Dimensional Dust Radiative Transfer Models

\subsection{RESULTS}

\subsubsection{Simulated Images}

Images of the clusters at several stages along the geometric sequence are shown in Figure 2.2 in near-IR (J, H, and K bands), mid-IR [Spitzer IRAC 3.6 $\mu \mathrm{m}, 4.5 \mu \mathrm{m}$, and $8.0 \mu \mathrm{m}$ bands; 58], and far-IR [Spitzer MIPS $24 \mu \mathrm{m}, 70 \mu \mathrm{m}$, and $160 \mu \mathrm{m}$ bands; 160] light from left to right. They were created from the SFE $=10 \%$ models with an outer radius of $50 \mathrm{pc}$, clumpy dust fraction of 0.9 and inner radii of $1,5,20$, and $45 \mathrm{pc}$ from top to bottom. 

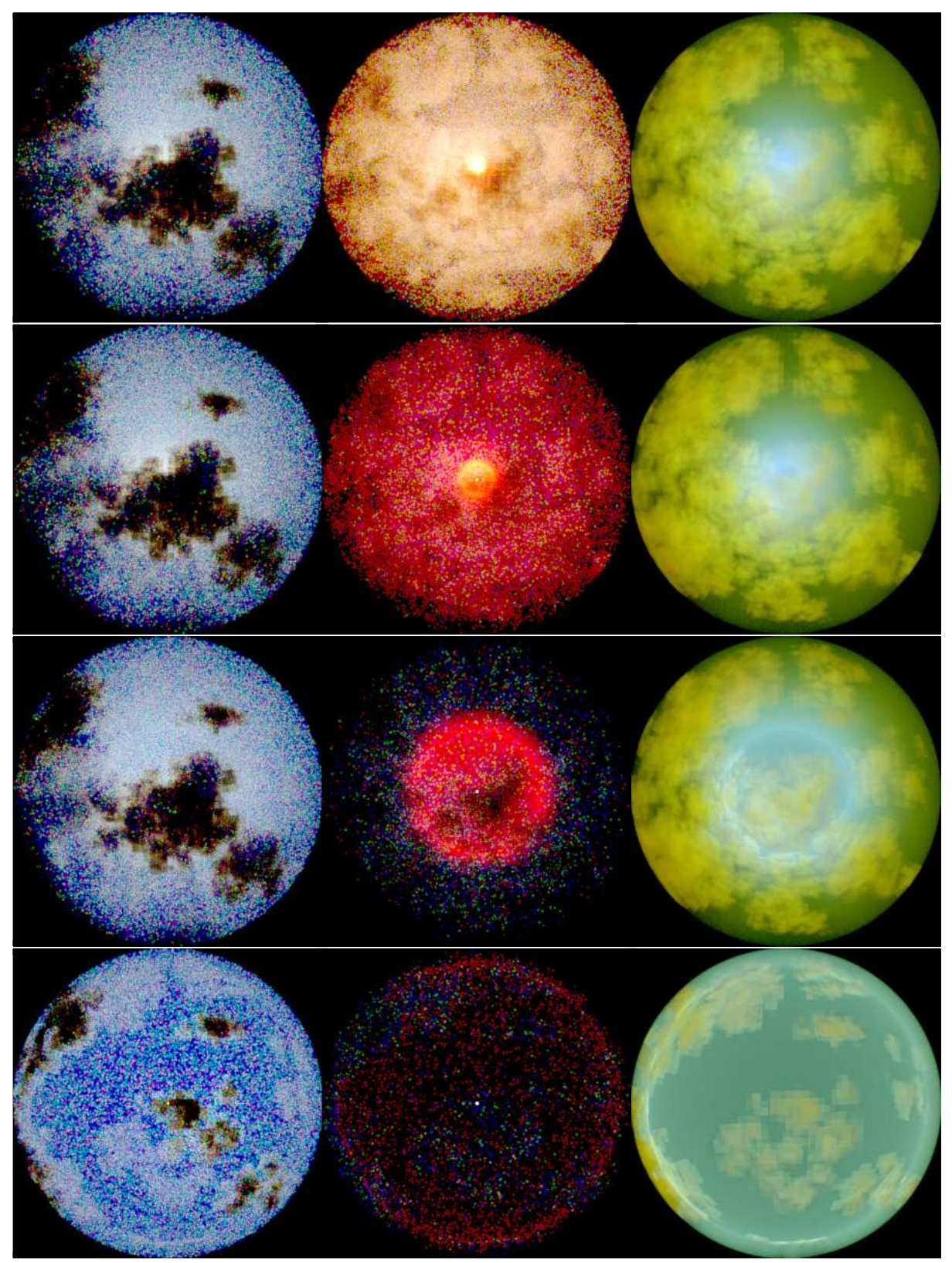

FiguRE 2.2: Simulated color images for a geometric (pseudo-evolutionary) sequence of a natal SSC for a SFE of $10 \%$, outer radius $=50 \mathrm{pc}$, and clumpy fraction $=90 \%$. Images are shown for an inner radius of the cocoon of $1 \mathrm{pc}$, $5 \mathrm{pc}, 20 \mathrm{pc}$, and $45 \mathrm{pc}$, from top to bottom respectively. The colors shown are for combinations of $\mathrm{J}, \mathrm{H}$, and $\mathrm{K}$ (left), IRAC $3.6 \mu \mathrm{m}, 4.5 \mu \mathrm{m}$, and $8 \mu \mathrm{m}$ (middle), and MIPS $24 \mu \mathrm{m}, 70 \mu \mathrm{m}$, and $160 \mu \mathrm{m}$ (right). 
One of the striking features of these simulations is that a significant amount of the near-IR light can escape the clumpy envelope, as seen by the scattered light on the inside of the cloud surfaces. Due to the scattered light, the near-IR images appear blue in color, because scattering favors bluer photons (in this case, J-band photons are scattered more regularly than K-band photons). The mid-IR band images, sensitive to warm dust and PAH/VSG emission, appear red because of the dominant PAH feature and high dust continuum in the $8 \mu \mathrm{m}$ band. As the inner radius moves outward, the MIR colors become more red because the inner dust temperature becomes lower, removing what little hot dust radiation in the smallwavelength bands there was. Additionally the hole appears to be the brightest feature in the mid-IR because the shell is being lit up by the central source. The far-IR bands probe both sides of the infrared dust peak, with the $24 \mu \mathrm{m}$ emission showing hotter dust and 70 and $160 \mu \mathrm{m}$ bands showing cold dust emission. Since the dust peak is typically near $70 \mu \mathrm{m}$, these images therefore appear green.

\subsubsection{The Change in Ultraviolet Flux with Clumpy Dust}

\section{Fraction}

Motivated by observations suggesting that roughly $50 \%$ of the UV flux leaks from embedded star clusters [see 102, 159, for details on the observations], we plot the fraction of UV light lost as a function of the $\mathrm{R}_{\text {in }} / \mathrm{R}_{\text {out }}$ ratio for the $99 \%$ clumpy models in Figure 2.3. 


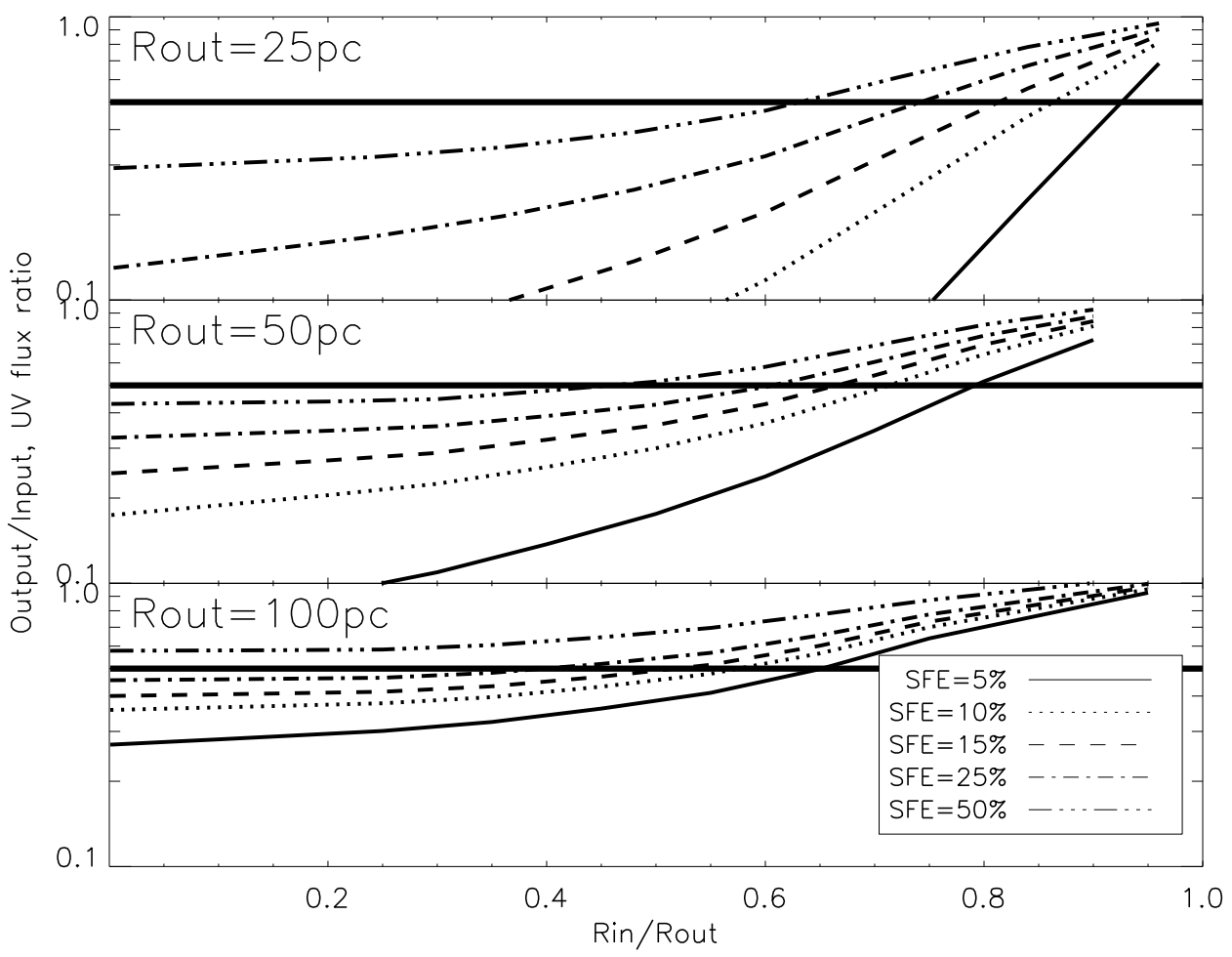

Figure 2.3: The fraction of UV light that escapes from the $99 \%$ clumpy embedding envelope as a function of $\mathrm{R}_{\text {in }} / \mathrm{R}_{\text {out }}$. The horizontal line is at a value of 0.5 , signifying $50 \%$ of the UV flux is not being reprocessed by the dust, and is only meant to help guide the eye. The solid line is for a SFE of $5 \%$, dotted line for $10 \%$, dashed line for $15 \%$, dash-dot line for $25 \%$, and dash-dot-dot for $50 \%$. 
Figure 2.3 shows that $R_{\text {in }} / R_{\text {out }}$ ratios $\gtrsim 0.5$ generally reproduce the results, depending on the $\mathrm{SFE}$ and $\mathrm{R}_{\text {out }}$ values adopted. The very high $\mathrm{SFE}$ values, such as $50 \%$, always allows a large fraction of the ultraviolet photons out of the envelope because of its low optical depth. The clumpiness will also have an effect on the fraction of UV light leakage, and generally less light will leak from envelopes with smaller clumpy fractions.

Since the radio sources studied in Reines et al. [159] and Johnson et al. [102] are obscure, however, their findings are probably for low-SFE, moderately evolved regions where the optical extinction for a corresponding model is about $A_{V} \sim$ $30-50$ on average. The UV light leakage would therefore take place along those sightlines exhibiting minimal extinction, $\mathrm{A}_{V} \lesssim 1$.

\subsubsection{Infrared Variation with Viewing Angle}

The clumpy dust distribution creates variations in infrared luminosity and certain spectral features depending on the viewing angle. In a general sense, clumpier media show the most variation in spectral features and luminosity compared to smoother media.

Figure 2.4 demonstrates how the infrared luminosity derived from a random sightline will be incorrect due to a clumpy envelope. The infrared luminosity inferred from a single sightline observation, plotted as stars in the figure and defined as the 3-1000 $\mu \mathrm{m}$ integrated luminosity, can vary from roughly half to nearly twice 

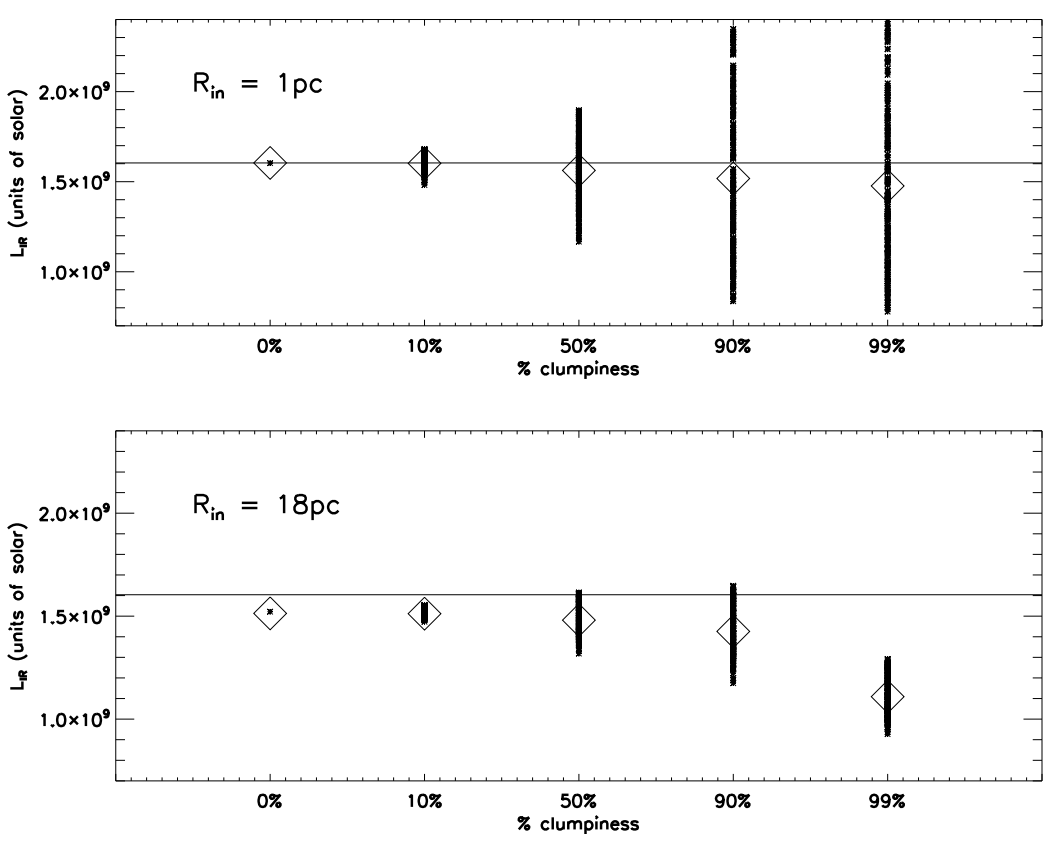

FiguRE 2.4: The computed infrared luminosity $(3-1000 \mu \mathrm{m})$ plotted versus the fraction of dust that is in clumps for an optically thick (top) and an optically thin (bottom) model. $\mathrm{R}_{\text {out }}$ is $25 \mathrm{pc}$ for both panels. The horizontal line is the input stellar cluster luminosity, which matches the $L_{I R}$ for optically thick models. The $L_{I R}$ computed along each sightline, assuming a spherical, smooth distribution, is plotted with stars, while the $L_{I R}$ values determined by adding up all sightlines for a model are shown as diamonds (this is the correct infrared luminosity). The clumpier models, as well as showing a larger dispersion in computed infrared luminosity values, also exhibit lower infrared luminosities in general, because the clumpy envelope allows ultraviolet photons to exit the envelope (see Figure 2.3).

the true infrared luminosity for a clumpy dust distribution. The true infrared luminosities are plotted as diamonds and are the infrared luminosities summed over all viewing angles for a model. For smooth dust distributions with thick envelopes, the input central source luminosity is equal to the true infrared luminosity. The infrared luminosities are compared to the stellar cluster's input luminosity of $1.604 \times 10^{9} \mathrm{~L}_{\odot}$, plotted as the solid horizontal line. 


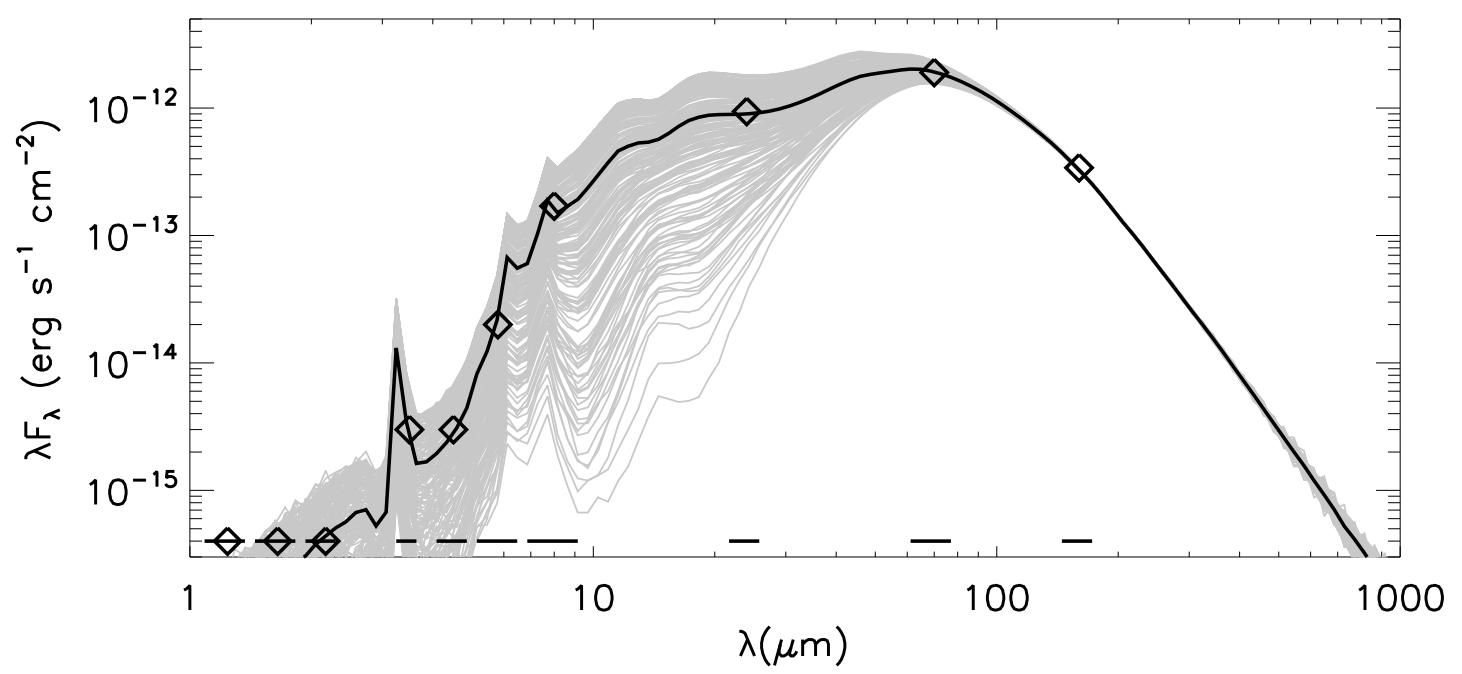

Figure 2.5: The variation of the SED for a given model due to the viewing angle of the observer. For this example, the model shown has $R_{\text {out }}=25 \mathrm{pc}$, $\mathrm{R}_{\text {in }}=3 \mathrm{pc}$, a clumpy fraction of 0.90 , and a $10 \%$ SFE. The mean SED is shown with a black line, and the near-IR and Spitzer observing bands are indicated with diamonds, with $\Delta \lambda$ for each band given at the bottom.

If a clump is along the line of sight in front of the central source, the optical depth will appear to be high and the 9.8 and $17 \mu \mathrm{m}$ silicate absorption features will be deeper in the infrared spectrum. However, clumps that are behind the central source will reflect light, and silicate emission features will therefore be evident. This is illustrated in Figure 2.5, which shows that the features of the infrared spectrum of a source inside a clumpy envelope are highly sightline-dependent. The average spectrum is also shown for comparison. 
Chapter 2. The Infrared Properties of Embedded Super Star Clusters: Predictions from Three-Dimensional Dust Radiative Transfer Models

\subsubsection{Spectral Energy Distribution Properties}

The output spectral energy distributions all generally have four components: (1) an extincted stellar spectrum from the central source; (2) thermal dust emission that peaks between 40 and $70 \mu \mathrm{m}$; (3) silicate emission or absorption features; and (4) the PAH features visible in the mid-IR. Figure 2.6 shows the smooth dust SEDs for a geometric sequence as an example of how these four main components are expected to change as the cluster evolves. The geometric sequences for all SFE and $\mathrm{R}_{\text {out }}$ values are given in Figures 2.7 and 2.8 for the smooth and $99 \%$ clumpy dust distributions. In Figure 2.8, the gray spectra are individual sightline SEDs for each $\mathrm{R}_{\text {in }}$ value, while the colors are the sightline-averaged values. 


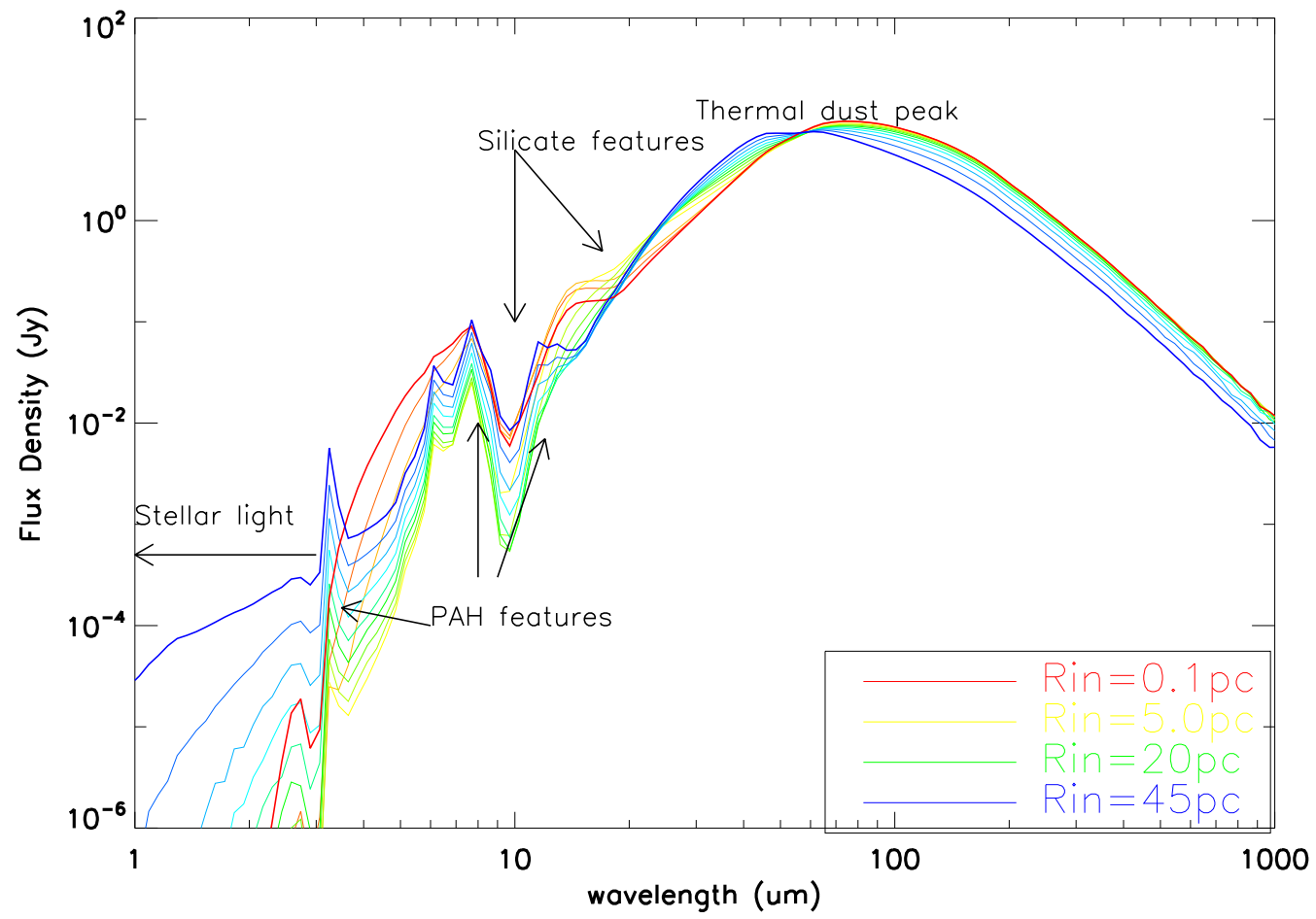

Figure 2.6: The spectra shown are along the geometric sequence in Table 1 for $\mathrm{R}_{\text {out }}=50 \mathrm{pc}$ and a smooth dust distribution. As $\mathrm{R}_{\text {in }}$ increases towards $\mathrm{R}_{\text {out }}$, the silicate features, most noticeably the one at $\sim 9.8 \mu \mathrm{m}$, become shallower. The PAH features are about the same throughout the sequence. The envelopes with small inner radii are more opaque to short-wave radiation and reprocess it to longer wavelengths. Therefore less flux between $1-5 \mu \mathrm{m}$ is measured and more flux at $100 \mu \mathrm{m}$, shifting the peak of the SED to the red. The stellar light is less extincted at larger $R_{i n}$ values. 


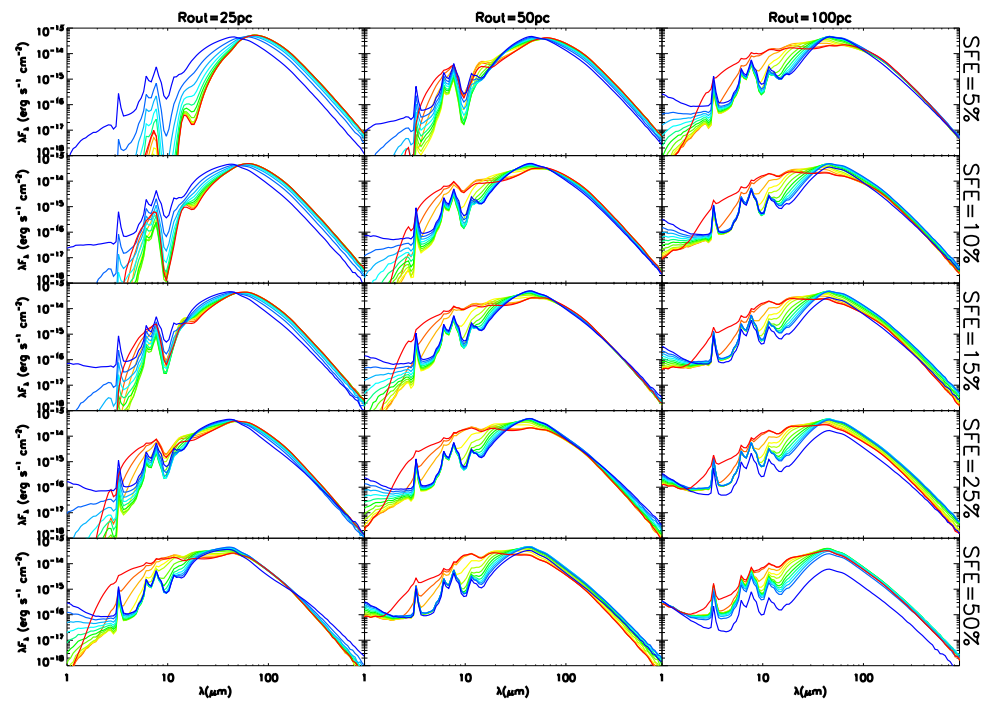

FiguRE 2.7: The infrared SEDs of all of the geometric sequences using a smooth dust distribution are shown; the range in $\mathrm{R}_{\text {in }}$ values from small to large are as in Figure 2.6.

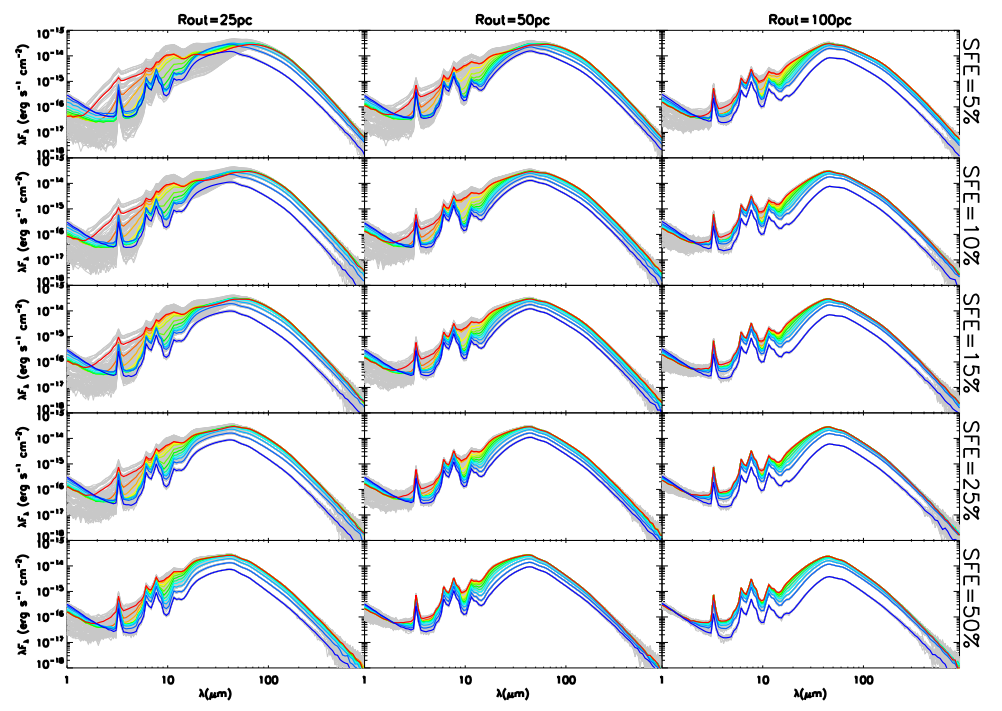

FIGURE 2.8: The infrared SEDs of all the geometric sequences using a $99 \%$ clumpy dust distribution, with the sightline-average data shown in colors as in

Figures 2.6 and 2.7 and the individual sightline data shown in gray scale. 
Chapter 2. The Infrared Properties of Embedded Super Star Clusters: Predictions from Three-Dimensional Dust Radiative Transfer Models

For the most dense models, including low $\mathrm{SFE}$ values and small $\mathrm{R}_{\text {in }}$ values, the starlight is largely absorbed by the envelope. For clumpy envelopes, many sightlines allow optical and UV photons to pass through so that a stellar continuum is visible, while the clumps have very high extinction values. For smooth dust distributions the starlight is largely absorbed along all sightlines. The $\mathrm{A}_{V}$ values for smooth distributions compared to the average values and ranges found in clumpy solutions, are listed in Table 2 for the $\mathrm{R}_{i n}=0.1 \mathrm{pc}$ models. The average $\mathrm{A}_{V}$ values for evolved stages in the geometric sequence are relatively small for all models (roughly less than 2), hence the spectra of clumpy and smooth models at high SFE value and large inner radii are similar. 
Table 2. Average and Range $\mathrm{A} V$ values for $\mathrm{R}_{\text {in }}=0.1 \mathrm{pc}$

\begin{tabular}{|c|c|c|c|c|c|c|}
\hline $\mathrm{SFE}(\%)$ & $\mathrm{R}_{\text {out }}$ & Smooth & $10 \%$ clumpy & $50 \%$ clumpy & $90 \%$ clumpy & $99 \%$ clumpy \\
\hline 5 & 25 & 490 & $474(441-569)$ & $411(245-885)$ & $348(49.1-1200)$ & $334(4.92-1270)$ \\
\hline 5 & 50 & 123 & 119 (111-143) & $103(61.4-221)$ & $87.0(12.3-300)$ & $83.4(1.24-318)$ \\
\hline 5 & 100 & 30.8 & $29.8(27.6-35.7)$ & $25.8(15.4-55.4)$ & $21.8(3.09-75.1)$ & $20.9(0.310-79.5)$ \\
\hline 10 & 25 & 232 & $225(209-270)$ & $195(116-419)$ & $165(23.2-569)$ & $158(2.34-603)$ \\
\hline 10 & 50 & 58.2 & $56.3(52.4-67.5)$ & $48.8(29.1-105)$ & $41.2(5.83-142)$ & $39.5(0.587-151)$ \\
\hline 10 & 100 & 14.6 & $14.1(13.1-16.9)$ & $12.2(7.30-26.2)$ & $10.3(1.47-35.6)$ & $9.88(0.147-37.7)$ \\
\hline 15 & 25 & 146 & $142(132-170)$ & $123(73.2-264)$ & $104(14.6-358)$ & $99.5(1.48-379)$ \\
\hline 15 & 50 & 36.7 & $35.5(33.0-42.5)$ & $30.7(18.3-66.1)$ & $26.0(3.68-89.6)$ & $24.9(0.369-94.8)$ \\
\hline 15 & 100 & 9.18 & $8.88(8.27-10.7)$ & $7.69(4.60-16.5)$ & $6.50(0.925-22.4)$ & $6.23(0.0925-23.7)$ \\
\hline 25 & 25 & 77.5 & $75.0(69.7-89.9)$ & $64.9(38.7-140)$ & $54.9(7.76-190)$ & $52.7(0.781-201)$ \\
\hline 25 & 50 & 19.4 & $18.8(17.5-22.5)$ & $16.3(9.71-35.0)$ & $13.7(1.95-47.4)$ & $13.2(0.196-50.2)$ \\
\hline 25 & 100 & 4.87 & $4.71(4.38-5.65)$ & $4.08(2.44-8.76)$ & $3.44(0.490-11.9)$ & $3.30(0.0490-12.6)$ \\
\hline 50 & 25 & 25.8 & $25.0(23.2-30.0)$ & $21.7(12.9-46.6)$ & $18.3(2.59-63.2)$ & $17.6(0.260-67.0)$ \\
\hline 50 & 50 & 6.48 & $6.27(5.83-7.52)$ & $5.43(3.25-11.7)$ & $4.59(0.652-15.8)$ & $4.40(0.0652-16.7)$ \\
\hline 50 & 100 & 1.63 & $1.58(1.47-1.89)$ & $1.36(0.816-2.93)$ & $1.15(0.163-3.96)$ & $1.10(0.0163-4.20)$ \\
\hline
\end{tabular}

The wavelength of the far-IR peak of the thermal grain emission depends on the predominant temperature of the dust in a model envelope. For the high column density envelopes, which have low SFE values and small $R_{\text {in }}$ values, the UV light is absorbed in the inner portions of the envelope so that much of the envelope remains quite cold. Therefore, low $\mathrm{SFE}$ values and small $\mathrm{R}_{\text {in }}$ values tend to produce 
infrared peaks at about $60 \mu \mathrm{m}$. For the optically thin models with high SFE values and large inner radii, a large proportion of the dust is being heated by the cluster, so the far-IR peak moves to shorter wavelengths.

Silicate absorption is directly proportional to the optical depth, so that the deepest silicate features appear in the largest column-depth models. Silicates in emission, as was described in $\S 2.4 .3$, appear in clumpy models where sightlines include dust clumps illuminated behind the central source, which re-emit absorbed starlight as $10 \mu \mathrm{m}$ light that is unblocked by any intervening clouds.

The PAH features are almost ubiquitous in the model SEDs. The early stages in the geometric sequence appear to have reduced emission from the PAHs for two reasons. The first reason is that for the high density models, there is very little volume from which the PAHs can be excited; PAHs are only excited by UV photons to a depth of $\mathrm{A}_{V}=1.5$. The second reason is because the thermal hot dust component dominates the mid-IR flux for the early stage models, which lowers the measured equivalent widths of these features. For later stages on the geometric sequence, the $\mathrm{PAH}$ emission is relatively constant along the sequence because as the ultraviolet flux on the inner surface of the envelope decreases as $\mathrm{r}^{-2}$, the surface area available to excite PAH molecules increases as $\mathrm{r}^{2}$. 


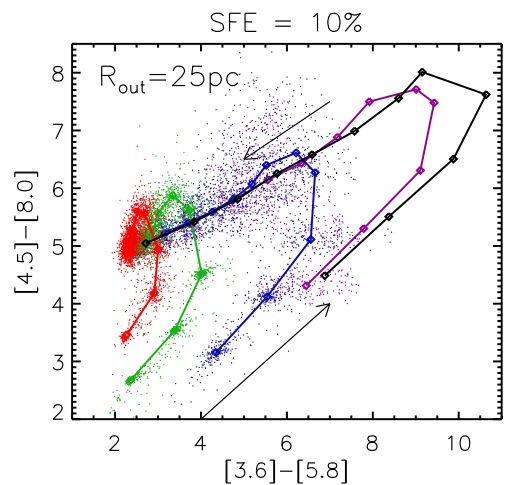

FIGURE 2.9: Using the mid-IR Spitzer/IRAC colors, the geometric sequence for $\mathrm{R}_{\text {out }}=25 \mathrm{pc}$ and $\mathrm{SFE}=10 \%$ is shown for smooth models (black line), 10\% clumpy models (purple), 50\% clumpy models (blue), 90\% clumpy models (green), and 99\% clumpy models (red). The geometric sequences begin towards the bottom with $\mathrm{R}_{\text {in }}=0.1 \mathrm{pc}$ and follow the arrows around the turn-around to their termination at $\mathrm{R}_{i n}=24 \mathrm{pc}$. The solid lines and diamonds trace out the sightline-averaged colors, and the dots represent individual sightlines. While even the individual average tracks often overlap, the different sightlines add a significant uncertainty to any interpretation of these data.

\subsubsection{Infrared Colors as Tracers of the Input Parameters}

The model SEDs have been convolved with numerous filter sets for comparison to photometric observations. These include the four IRAS bands $(12,25,60$ and $100 \mu \mathrm{m})$, three standard near-IR bands (J, H, and K), Spitzer IRAC bands (3.6,

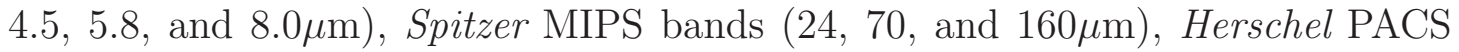
bands $(70,100$, and $160 \mu \mathrm{m})$ and Herschel SPIRE bands $(250,350$, and $500 \mu \mathrm{m})$.

As an example of how colors can be used to plot the data, we show the IRAC colors for the $\mathrm{R}_{\text {out }}=25 \mathrm{pc}$ and $\mathrm{SFE}=10 \%$ models for all clumpiness values and all $\mathrm{R}_{\text {in }}$ values in Figure 2.9. There are degeneracies in the model average values at late evolutionary stages, and the individual sightlines make the interpretation of data extremely difficult. 
Because the clumpy dust distributions create degeneracies in the SEDs, diagnostics to recover the input parameters were developed using the smooth dust distribution models first. In Figure 2.10 the [3.6]-[24] color is plotted against the $\mathrm{R}_{\text {in }} / \mathrm{R}_{\text {out }}$. Red, green, and blue are used to designate models with different $\mathrm{R}_{\text {out }}$ values (red, green, and blue for 100, 50, and 25pc, respectively), and the plotting symbols are for the five different SFE values considered. Although there is significant overlap between the colors, the tracks are separate over most of the $R_{\text {in }} / R_{\text {out }}$ values for each individual SFE value. The [70]-[160] color can be used to roughly determine the SFE value as shown in Figure 2.11; the bars represent the model dispersions. There is significant overlap between dispersion in SFE values of $10 \%$ to $25 \%$, but [70]-[160] values of $>0.9$ represent very low SFE values $(<10 \%)$, values between $\sim-1.0$ and -1.5 represent SFE values between $10 \%$ and $25 \%$, and values $>-1.6$ will be for SFE of $50 \%$ or greater. 


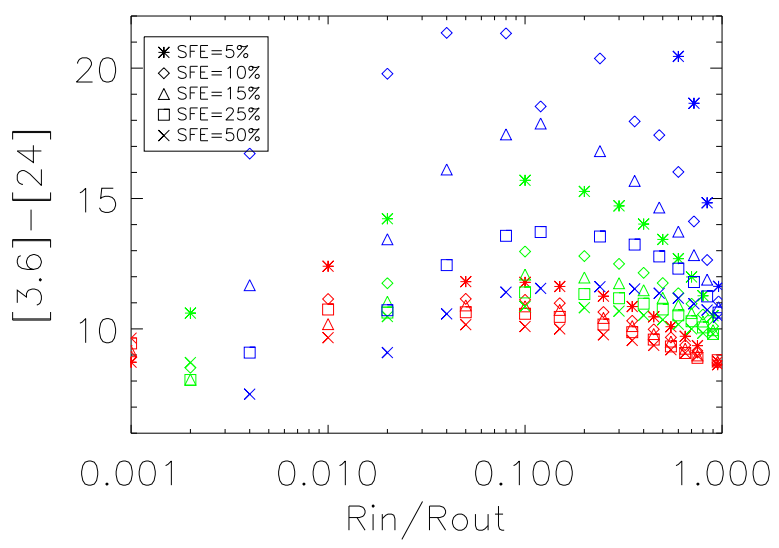

FiguRE 2.10: The [3.6]-[24] color is plotted versus the ratio of the inner and outer envelope radii for the three different outer radius values considered, 25 , 50 , and 100pc (shown in blue, green, and red, respectively) using the smooth dust distribution models only. If the star formation efficiency (SFE) can be determined using the [70]-[160] color as shown in Figure 2.11, then the $\mathrm{R}_{\text {in }} / \mathrm{R}_{\text {out }}$ value can be estimated using this plot.

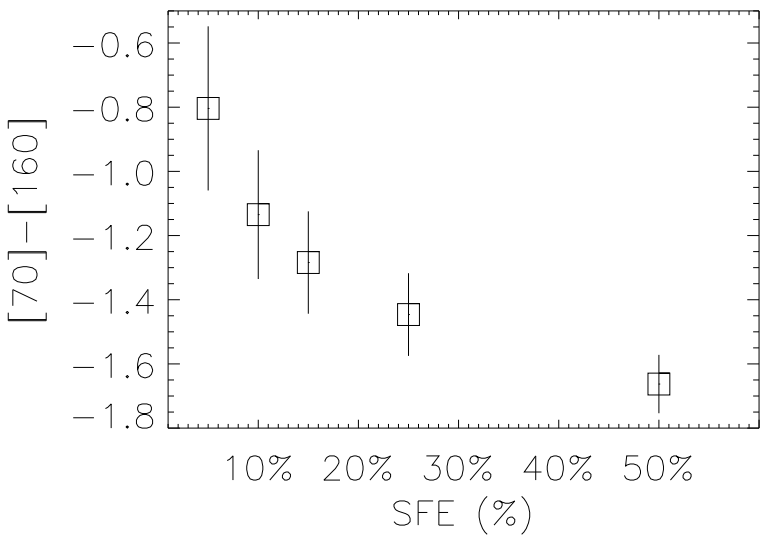

Figure 2.11: The [70]-[160] color is used to separate different SFE values for the models with a smooth dust distribution. The bars shown are the dispersion and there is a fair bit of overlap between the models. One can only expect, therefore, to be able to roughly determine the SFE for the smooth models. 
Colors that can best be used to recover the input parameters for the entire, clumpy suite of models were also investigated. Given that the value of the input parameters and the viewing angle can both significantly change an embedded source's SED and colors, there is a need to determine which, if any, photometric measurements can be used to reliably constrain the physical geometry. For a given input parameter (SFE, clumpiness, $\mathrm{R}_{\text {in }}$, or $\mathrm{R}_{\text {out }}$ ), the following calculation was made: for each color, the mean color and standard deviation was measured with the input parameter fixed and everything else (i.e. the other input parameters and all viewing angles) variable. This was done at each fixed input parameter value (e.g. for each of the five different SFE values). Finally, the difference in the means is divided by the greatest of the standard deviations - this is a measure of how much the input parameter affects the color, compared to the other input parameters and the viewing angle ambiguities.

This analysis is related to a Principal Component Analysis, but adjusted to our goal of finding the minimal set of colors with maximal physical diagnostic power. Rather than solving freely for eigenvectors of the model set in color space, which may not directly correspond to the physical variables, we hypothesize that a principal component exists for which the eigenvalue would directly correspond to a physical parameter, and then measure the projection of that hypothetical component onto each of our color axes. This process allows of course that there is no such 'physically diagnostic principal component,' in which case the variation of all colors with the physical parameter would be small compared to the standard 


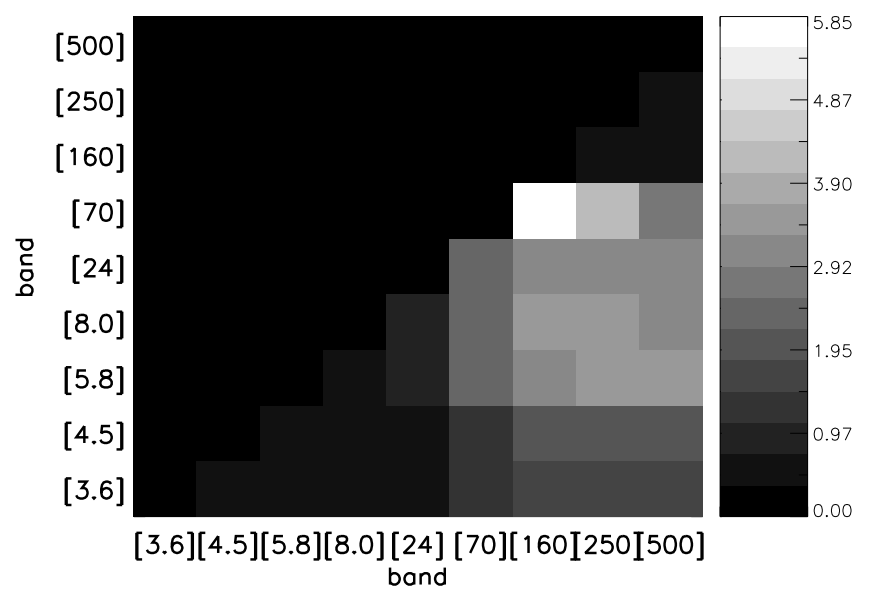

Figure 2.12: Determining the best color (y-axis magnitude minus $\mathrm{x}$-axis magnitude) for recovering the star formation efficiency (SFE). In order to determine the best color for discriminating between different SFE values, this calculation was made: the spread in the mean color values between the different $\mathrm{SFE}$ values was divided by the greatest standard deviation of each SFE value was computed. The maximum resulting value is the most discriminating color (see §2.4.5). Each color is the $\mathrm{y}$-axis magnitude minus $\mathrm{x}$-axis magnitude, and the best color was found to be [70]-[160]. How well this 'best' color helps to determine the SFE is shown in Figure 2.15, and discussed fully in $\S 2.4 .5$.

deviation in the color due to other causes.

Figures 2.12 to 2.14 are plots of infrared color versus infrared color where the grayscale shows the value of the quotient for determining the best diagnostic color described above. Values in the figures near zero indicate little spread in the average color for each input parameter value, while values at the maximum have relatively large spreads in average color values across the input parameter values, as well as small standard deviations from the mean. These are therefore the best colors for separating input parameter values: (1) [70]-[160] for SFE (Figure 2.12); (2) [3.6][5.8] for fraction of dust in a clumpy distribution (Figure 2.13); and (3) [8.0]-[24] for both $\mathrm{R}_{\text {out }}$ and the $\mathrm{R}_{\text {in }} / \mathrm{R}_{\text {out }}$ ratio (Figure 2.14 ). 


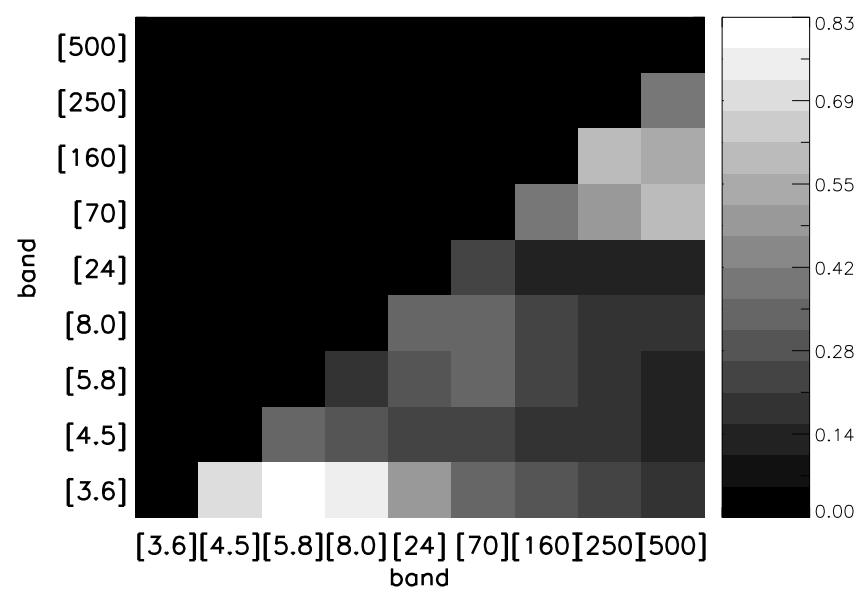

FiguRE 2.13: Determining the best color for recovering the fraction of dust in a smooth distribution in the envelope. Using the criterion described in $\S 2.4 .5$, the best color for recovering the percentage of dust that is smooth was found to be [3.6]-[5.8]. However, as shown in Figure 2.16, even this 'best' color cannot be used to discriminate between the average values.

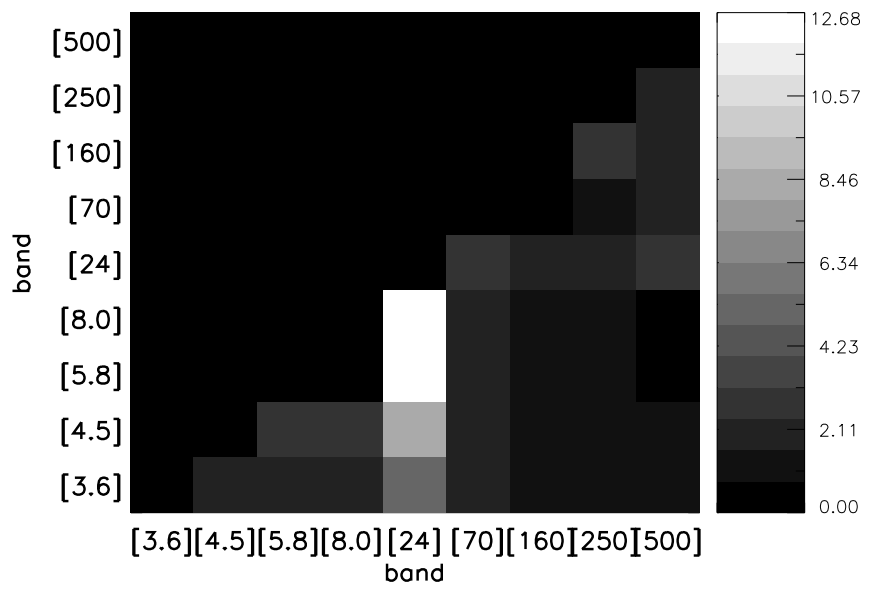

Figure 2.14: Determining the best color for recovering the $\mathrm{R}_{\text {out }}$ ratio. The best discriminator found for $\mathrm{R}_{\text {out }}$, which also well separates and $\mathrm{R}_{\text {in }} / \mathrm{R}_{\text {out }}$ ratios, is [8.0]-[24], and is shown in Figure 2.17. 
Figures $2.15,2.16$, and 2.17 plot these most useful colors' average values and standard deviations for the whole suite of models versus the input parameters. For SFE (Figure 2.15), as with the smooth dust distribution models, there is overlap in the [70]-[160] color between the various SFE values. The overlap is still most significant between 10 and 25\%, for which color values between -1.5 and -0.9 would all be valid. Color values above -0.9 match the $5 \% \mathrm{SFE}$, and color values below -1.5 match the $50 \%$ SFE. For the smooth dust fraction, within the error bars in the [3.6]-[5.8] versus smooth dust fraction plot (Figure 2.16) the color values are still degenerate, and using thermal radio data [such as was presented in $159]$ is a more reliable metric for determining the fraction of ultraviolet light lost from an embedded star cluster. However, because the standard deviations in color of the clumpiest fractions are small compared to the smooth models (due to the large differences in the input parameter values being explored), a large, statistical study of embedded star clusters, for which average color values and standard deviations could be computed, could potentially be used to discern between the different smooth dust fraction values. For $\mathrm{R}_{\text {in }} / \mathrm{R}_{\text {out }}$ (Figure 2.17 ), colors are still intractable for small $\mathrm{R}_{\text {in }} / \mathrm{R}_{\text {out }}$ values. For $\mathrm{R}_{\text {in }} / \mathrm{R}_{\text {out }}>0.4$, the [8.0]-[24] can be used both to distinguish between different $\mathrm{R}_{\text {in }} / \mathrm{R}_{\text {out }}$ values and to distinguish between the different $\mathrm{R}_{\text {out }}$ values. 


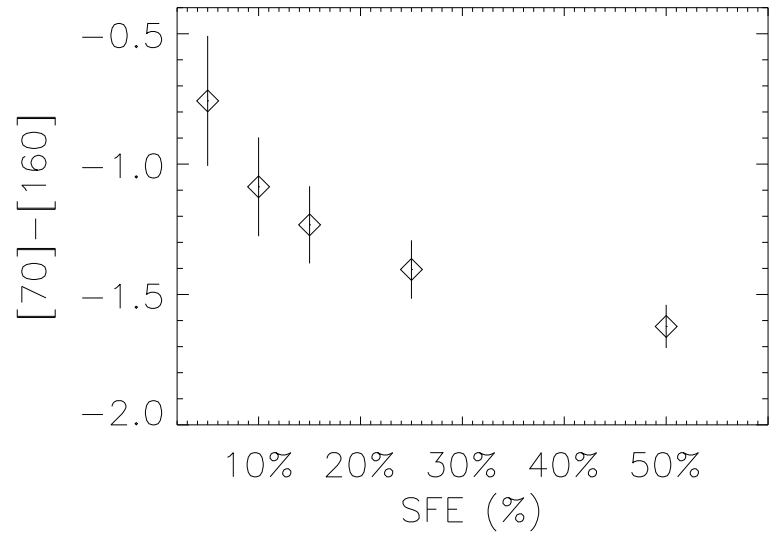

Figure 2.15: The [70]-[160] color that can best be used to recover the SFE value. The diamonds are the average values over the entire dataset, and the error bars represent the one- $\sigma$ errors at each SFE value.

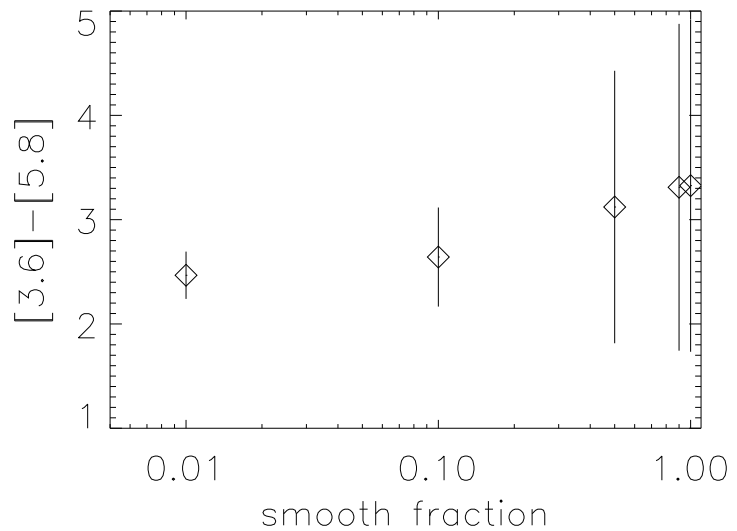

Figure 2.16: The [3.6]-[5.8] color that was found to best recover the fraction of dust that is smoothly distributed in the envelope. Given the $1-\sigma$ error bars for the smoothest models (i.e. there is such a great diversity in this color due to the input parameters) it is only possible to separate the smooth from the clumpy models in a statistical sample where the standard deviation in the color could be measured. 


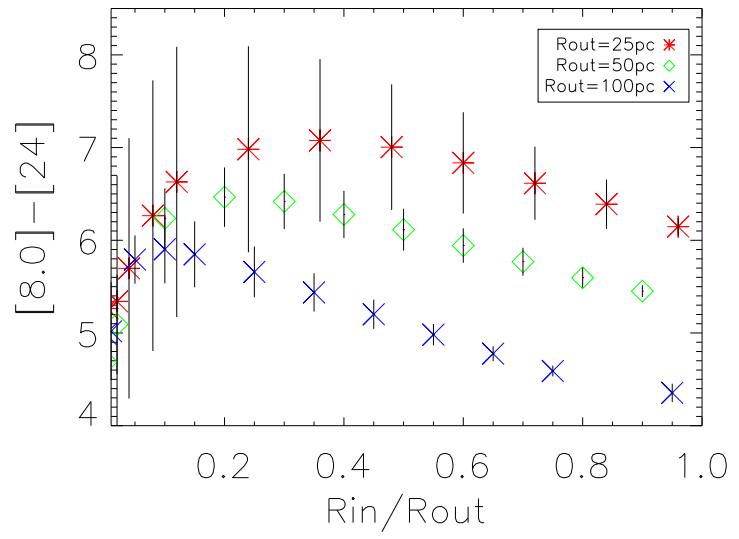

FiguRE 2.17: For envelopes with $\mathrm{R}_{\text {in }} / \mathrm{R}_{\text {out }} \gtrsim 0.4$ the [8.0]-[24] color can be used to determine both the $\mathrm{R}_{\text {out }}$ value, because they are well separated from each other, and the $\mathrm{R}_{\text {in }} / \mathrm{R}_{\text {out }}$ ratio. 


\subsection{COMPARISONS TO OBSERVATIONS}

In the Milky Way, individual embedded massive stars are surrounded by what have been identified as "Ultracompact HiI Regions" (UCHIIs), and it is hypothesized that the constituent massive stars in embedded SSCs will also be surrounded by UCHII regions. For this reason we compare our models to a sample of resolved embedded massive stars in order to test whether our models have reproduced, to first order, the necessary features of embedded massive stars. The sample is an IRAS sample studied in Wood \& Churchwell [224], in which it was found that $60 \%$ of the brightest IRAS sources $\left(>10^{4} \mathrm{Jy}\right.$ at $\left.100 \mu \mathrm{m}\right)$ in the color range of their galactic survey are UCHII regions. Wood \& Churchwell [224] also found that galactic UCHıI regions strictly obey a set of color criteria in the infrared with $\log \left(F_{60 \mu m} / F_{12 \mu m}\right)>1.30$ and $\log \left(F_{25 \mu m} / F_{12 \mu m}\right)>0.57$, while very few other types of objects had IRAS colors fitting these criteria. Therefore, these color criteria appear to be relatively robust for identifying UCHıI regions, and we might expect natal SSCs to obey these criteria as well.

We have convolved our model results with the IRAS filter profiles, and Figure 2.18 shows the IRAS colors from a subset of our models compared to field objects (plus signs) and UCHıI regions (diamonds) from the Kurtz, Churchwell, \& Wood [111] survey (which includes the Wood \& Churchwell [224] sample). In this figure, we focus on only the family of models with a $10 \%$ stellar mass component and a relative fraction of clumpy dust of $90 \%$, although the other model families have 


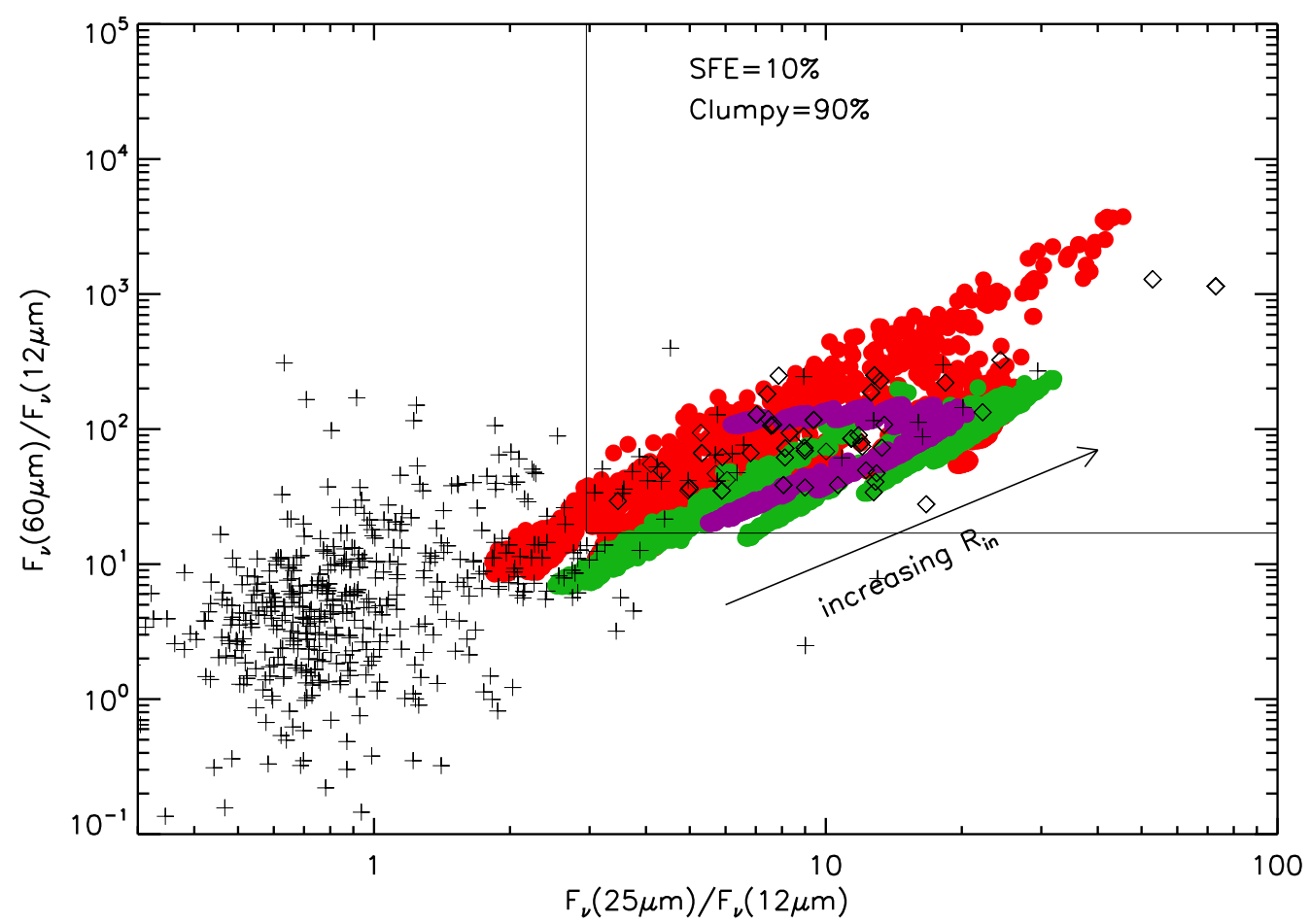

Figure 2.18: The IRAS colors from a subset of our models assuming $10 \% \mathrm{SFE}$ and clumpy fraction $=0.9$ compared to field objects (plus signs) and UCHII regions (diamonds) from the Kurtz, Churchwell, \& Wood [111] survey. Models with $R_{\text {out }}=25 \mathrm{pc}$ are shown as red points, $R_{\text {out }}=50 \mathrm{pc}$ as green points, and $R_{\text {out }}=100 \mathrm{pc}$ as purple points. The values of $R_{\text {in }}$ used are those shown in

Table 1, and all sight lines are shown for each set of model parameters.

IRAS colors that are included in the range represented by the models shown.

Models with $\mathrm{R}_{\text {out }}=25 \mathrm{pc}$ are shown as red points, $\mathrm{R}_{\text {out }}=50 \mathrm{pc}$ as green points, and $\mathrm{R}_{\text {out }}=100 \mathrm{pc}$ as purple points. The values of $\mathrm{R}_{\text {in }}$ used are those shown in Table 1, and all sight-lines are shown for each set of model parameters, which produces additional scatter. 
Chapter 2. The Infrared Properties of Embedded Super Star Clusters: Predictions from Three-Dimensional Dust Radiative Transfer Models

There is good agreement between our models and the UCHIIs sample shown in Figure 2.18. There are a few of points to lower left and upper right that are not traced by our models. The lower left points have very hot dust in the inner portions of their envelopes, and might be modeled with a smaller $\mathrm{R}_{\text {in }}$ value that puts the hot dust closer to the heating source. The points to upper right seem to be an extension of the basic trend of increasing $R_{i n}$. It is therefore possible that we could reproduce these UCHIIs if we simply assumed a lower mass cutoff to our cluster which would lower the UV flux incident on the inner envelope.

\subsection{KNOWN LIMITATIONS}

Here we discuss some of the limitations of the models as a guide for future improvements.

(1) The models assume that the central star cluster is a point source. While optical measurements have discovered half-light radii of super star clusters of $1.7 \mathrm{pc}$ on average [127], which is significantly smaller than the assumed outer envelope radii, it is nevertheless more realistic to place several ultracompact HiI regions at the center of the envelope (see Figure 2.1). The intracluster dust would be heated at early evolutionary stages if multiple, smaller clusters were used, creating a hotter dust component than is seen with the current models. 
(2) Variations in metallicity have not been considered. For small changes in metallicity (i.e. down to half or third solar metallicity), we expect the dust-to-gas ratio to scale with metallicity. However, more extreme environments, like blue compact dwarf galaxies, where metallicities are often much lower than this, show dust in excess to what is expected from their metallicity alone (T. X. Thuan, private communication). Accounting for very low metallicities is therefore a complicated issue that is probably best handled on an individual basis.

(3) The evolutionary sequences presented in this paper do not allow the central star cluster to evolve. As described in $\S 2.3 .5$, the time between cluster formation and the dispersal of the embedding envelope is short, on the order of $3-4 \mathrm{Myr}$. However, the dispersal rate is not well known, so we have not made any attempt to place time stamps on the evolutionary sequence presented here. Hydrodynamical models are needed to answer this problem, and several hydrodynamical and semianalytical studies of super star clusters already exist [e.g. 201, 202, 227, 228]. The rate at which the envelope is dispersed could potentially be answered by similar studies of a super star cluster's embedded phase.

(4) This work has concentrated solely on the dust emission and absorption in embedded super star clusters. Inclusion of nebular line emission in the model spectra could be useful if line diagnostics could be identified that break the model degeneracies.

(5) The models do not include an interstellar radiation field (ISRF) incident on 
the outside of the dusty envelope. An ISRF would heat the dust on the outside of the envelope and provide an additional component to the starlight visible in the UV/optical/near-IR regime. This could affect the [3.6]-[5.8] color used to distinguish between different fractions of clumpy dust. The heating of the outer envelope could affect the far-IR fluxes as well.

\section{$2.7 \quad$ SUMMARY}

We present SEDs and colors of embedded SSCs created using spherical threedimensional models. By varying the input parameters according to a series of evolutionary sequences, we have created a suite of models that can be used to constrain the evolutionary state of an embedded super star cluster. The main conclusions of the study are:

(1) A hierarchically clumped medium is suitable for recreating the porous environments observed around embedded super star clusters [e.g. 102];

(2) The infrared luminosity derived from a single sightline observation of a clumpy envelope can be wrong by as much as a factor of two from the true value;

(3) The infrared SED also varies with sightline in these clumpy models, which has an impact on the near- and mid-infrared colors and magnitudes, the strength of the observed silicate features, and the dust continuum measured at these wavelengths; 
(4) For the smooth dust distribution models, the evolutionary sequences that begin with mid-infrared obscure envelopes $\left(\mathrm{A}_{V} \gtrsim 50\right)$ are marked by a gradual decline in the silicate absorption features at 9.8 and $17 \mu \mathrm{m}$ and a corresponding increase in the visual and ultraviolet flux as the cluster envelope evolves. Those sequences that begin with infrared-transparent envelopes $\left(\mathrm{A}_{V}<50\right)$ instead have a predominant hot dust component and silicates in emission at early stages that eventually both fade away as the inner envelope radius moves outward. The clumpy envelope acts to confuse these general trends, making it harder to determine envelope properties.

(5) Several diagnostic colors were found to constrain the envelope properties. The Spitzer MIPS [70]-[160] color is found to be a good diagnostic of the star formation efficiency, particularly at separating very low and high values (such as $5 \%$ and $50 \%$ ) from more moderate values (between $10 \%$ and 25\%). The [3.6]-[5.8] color can be used to determine the fraction of clumpy dust in the envelope for large samples of embedded super star clusters, but not for individual sources (see $§ 2.4 .5$ for details). In order to determine the $\mathrm{R}_{\text {in }}$ and $\mathrm{R}_{\text {out }}$ values, the [8.0]-[24] color can be used for $\mathrm{R}_{\text {in }} / \mathrm{R}_{\text {out }} \gtrsim 0.4$. Below this value the data is degenerate for all colors.

(6) The model IRAS colors trace the same area of color space as ultracompact HiI regions, the Galactic analogues to extragalactic embedded super star clusters, suggesting that the models will also be useful when data of resolved, embedded super star clusters become available. 


\section{Chapter 3}

\section{First Models of the Molecular}

\section{Emission from Embedded Super}

\section{Star Clusters}

\subsection{ABSTRACT}

As a follow-up to Whelan et al. [218], I have used available one-dimensional photodissociative chemistry and molecular line radiative transfer codes in order to predict the general molecular emission properties in embedded super star cluster environments. These models were intentionally kept simple because threedimensional codes are not yet clearly benchmarked. Our first results suggest that 
the cloud geometries assumed by Whelan et al. are too simplistic: constant density envelopes are superluminous in $\mathrm{CO}$ and $\mathrm{HCN}$ line emission, a problem that can be solved by introducing density power laws with values between -1 and -2 . Naturally these models cannot account for clumping in the embedding envelope. With input parameters of envelope mass, inner radius, and power law exponent, we constructed a suite of models that compare the integrated molecular line feature strength with samples of Milky Way giant molecular clouds as well as star-forming galaxies; super star clusters should fall between these two size regimes. The results match expectations well, but there are serious degeneracies that cannot be broken with the current one-dimensional implementation.

\subsection{INTRODUCTION}

Within the last two decades, multiple radio, infrared, and optical studies have informed us about the hot gas and young stellar populations associated with super star clusters (SSCs). The highest resolution studies have been in the optical [e.g. 142] and the radio [e.g. 100]. Additional information, particularly about the infrared luminosity and possible effects of clumping, has also been determined at infrared wavelengths $[184,205]$.

With the advent of the Atacama Large Millimeter/submillimeter Array (ALMA), high spatial resolution at millimeter wavelengths is going to allow us, for the first time, to view these dense star-forming environments in isolation from the diffuse 
emission that surrounds them. Little is known about the embedded phase of SSCs [see, e.g. 218]. We expect, however, that embedded SSCs will be enveloped by giant molecular clouds (GMCs) of relatively high density and pressure [101].

Studying the molecular properties of embedded SSCs requires photodissociative chemistry and molecular line radiative transfer codes, which are in turn dependent on knowledge about the dominant chemical networks at play in GMC environments as well as the Einstein coefficients for commonly studied molecules. This body of knowledge exists, and has been implemented into a number of different codes.

We will be using the CLOUDY $[1,62]$ and Ratran [78] codes in order to determine molecular line luminosities for embedded SSC environments. We will plot the line luminosity versus infrared luminosities determined with the same dust radiative transfer code as used in Whelan et al. [218] and compare the results for embedded SSCs to Milky Way GMCs [230] and whole star-forming galaxies [65]. We will discuss the codes and their uses in $\S 3.3$, present our first results in $\S 3.4$, present our current best parametrization of the cloud geometry in $\S 3.5$, and end with concluding remarks about future work $(\S 2.7)$. 


\subsection{Chemical and Radiative Transfer Codes}

The CLOUDY 90 code [62] was originally developed to numerically simulate plasmas. Ionization balance being a critical key to a properly running plasma simulation, it was not long before CLOUDY was extended to include neutral gas, including molecular gas produced via a chemical network hosting 1000 reactions [1]. The chemical network in CLOUDY rivals other special-purpose chemical network codes in size and allowable input physics [165], and has the distinction of being one of the few codes available that works in spherical geometries. These combined capabilities make CLOUDY one of the most powerful tools for determining molecular abundances in GMCs that are irradiated by young SSCs.

Once the chemical network has been solved for, the molecular abundances as a function of envelope radius can be input to Ratran [78]. The public version on Ratran provides one-dimensional radiative transfer through molecular clouds, taking into account the density and optical depth of the gas so that collisional excitations can be realistically computed. The code functions efficiently with an accelerated $\Lambda$ iteration technique that uses a number of photons that is suitable for the required signal-to-noise, and iterates until the requested accuracy is reached (typically some thousandths to millionths of the output value). The code was chosen for its relative ease of use, and its capability of dealing with optically thick molecular transitions. 
One error was found in Ratran during the late stages of the project. The Einstein coefficients are taken from the LAMDA database ${ }^{1}$, where, for instance, the collisional rates of $\mathrm{CO}$ with $\mathrm{H}_{2}$ are known to be different depending on whether the $\mathrm{H}_{2}$ is in the para- or ortho- state (the ortho-to-para ratio is an input). However, when the output line luminosity is being computed, the optical depth through dust (the dust-to-gas ratio is also an input) is computed assuming that the dust density is a function of the ortho- $\mathrm{H}_{2}$ density only (not the entire $\mathrm{H}_{2}$ density. Therefore, the optical depths are low by roughly a factor of 2-4 and the output luminosities are high by a similar factor. This error can be corrected simply by increasing the input dust-to-gas ratio in the code, but as we will see, this relatively small error does not adversely effect the results.

\subsection{First Results: Computations Using Old Ge-}

\section{ometry}

The models grid that was computed in Whelan et al. [218] was employed for these models. For the same densities and envelope sizes, molecular line radiative transfer was done for $\mathrm{CO}$ and HCN. The gas-to-dust ratio was assumed to be 100, which is a standard value for the ISM, and the relative molecular abundances of $\mathrm{CO}$ and $\mathrm{HCN}\left[10^{-4}\right.$ and $5 \times 10^{-9}$ respectively; 155,200$]$.

\footnotetext{
${ }^{1}$ http://home.strw.leidenuniv.nl/ moldata/
} 
One additional unknown was the value of the kinetic gas temperature, a variable that regulates the collisional rate between $\mathrm{CO}$ and $\mathrm{H}_{2}$, its primary collisional partner (and the only one employed on the basis of relative abundance). In Figure( 3.1, models that force the kinetic gas temperature to values between $15 \mathrm{~K}$ and $50 \mathrm{~K}$, a range of suitable answers considering the probable temperature of GMCs from observations [49]. For the three outer radii tested for, the spread in possible values is as much as two factors. Furthermore, when the kinetic gas temperature is set to the dust temperature solved for with dust radiative transfer, the value is always about average. Since the gas and dust are expected to be in relative thermal equilibrium in a GMC, this answer was employed for future models.

For the full 'evolutionary tracks' at $\mathrm{R}_{\text {outer }}=25,50$, and $100 \mathrm{pc}$ with envelope masses of $9 \times 10^{6} \mathrm{M}_{\odot}$, the values of the infrared and CO $(J=1-0)$ line are shown in Figure 3.2. The arrows indicated increasing values of $\mathrm{R}_{\text {inner }}$. At later stages, when the envelope is very thin, a significant fraction of the heating photons from the central source are able to leave, so the infrared luminosity decreases. Likewise, track leads to smaller and smaller CO luminosities because the mass of CO is decreasing. These models show quite clearly that for these model geometries, the CO line luminosity is primarily attributable to CO mass (hence the change in luminosity down separate tracks) and density (hence the significantly different values for different $R_{\text {outer }}$ values). This result is none too surprising, since the line flux is dependent on the collisional rate and the optical depth through the cloud.

When these model results are compared with the galaxy sample from Gao \& 


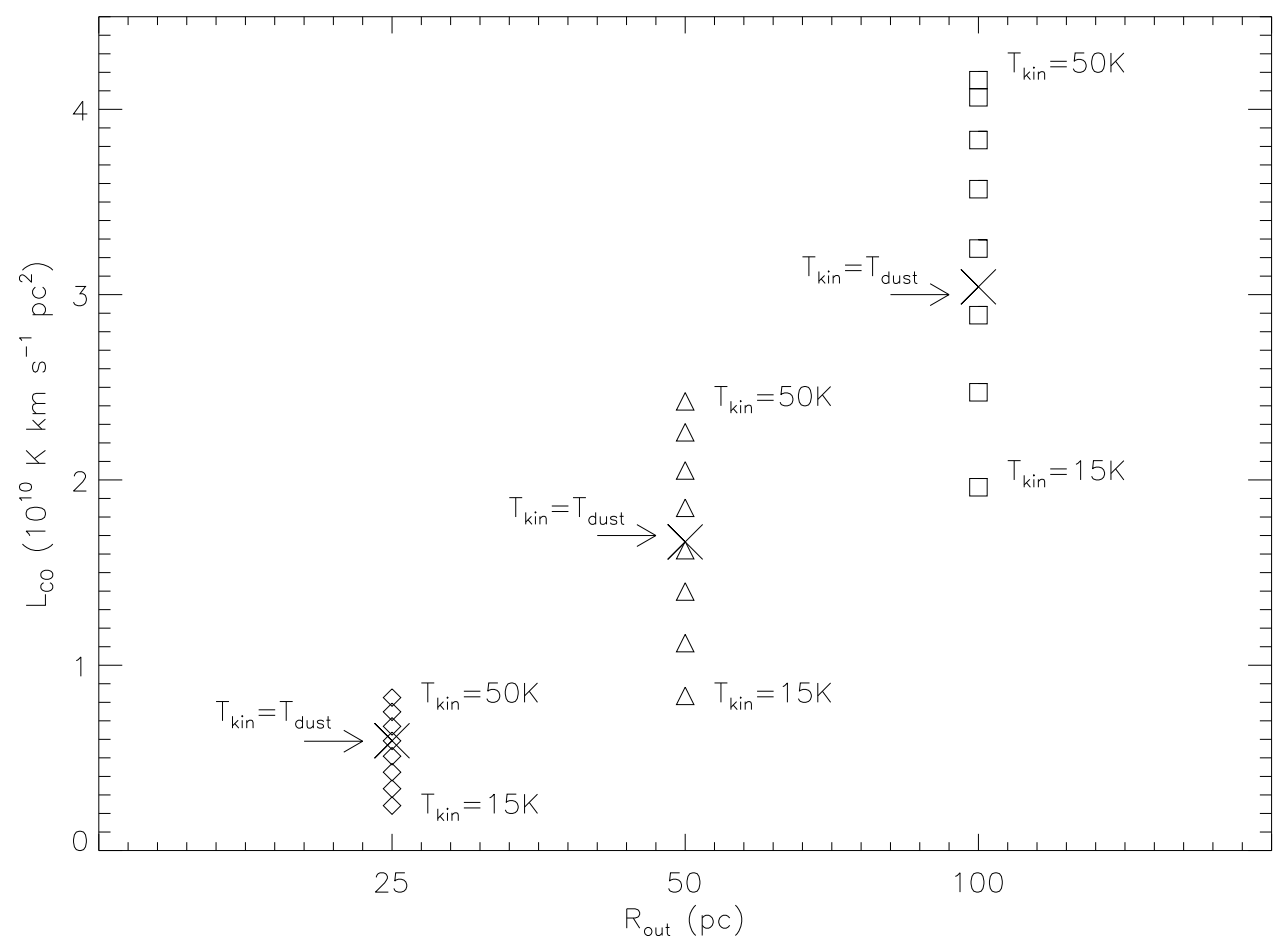

Figure 3.1: The luminosity of the $\mathrm{CO}(J=1-0)$ line is shown for a variety of kinetic temperatures for three models with outer envelope radii of 25,50 , and $100 \mathrm{pc}$. The value computed for when the kinetic temperature was forced to follow the computed dust temperature in the envelope is shown with $\times$ 's and is the value finally decided upon.

Solomon [65], we see that the embedded SSC models lie well off of the trend determined for those galaxies (Figure 3.3, 3.4). In fact, the embedded SSC CO and HCN luminosities are superluminal by between two and three orders of magnitude. If this were the case, the embedded SSCs would outshine their host galaxies in CO; this result must be incorrect.

Hydrodynamical simulations of Galactic molecular clouds show that collapsing clouds produce clear density gradients towards their center [185]. By implementing such a solution for embedded SSCs, we are able to place a large amount of the 


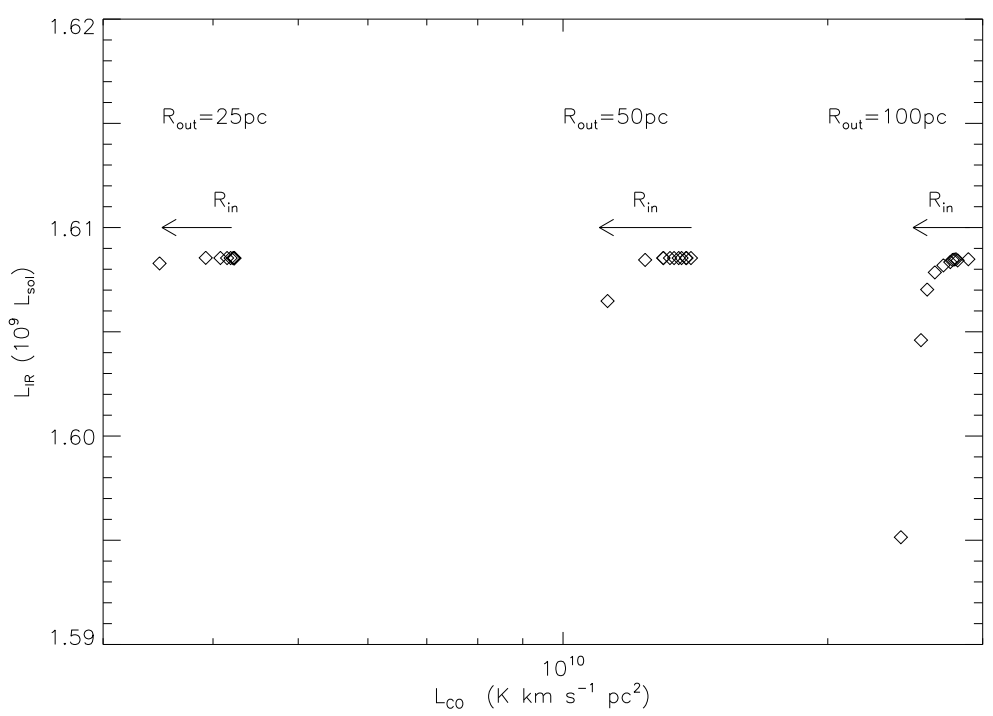

Figure 3.2: The total infrared line luminosity is plotted versus the $\mathrm{CO}(J=1-0)$ line luminosity for an initial envelope mass of $9 \times 10^{6} \mathrm{M}_{\odot}$ along the evolutionary tracks for $\mathrm{R}_{\text {outer }}=25,50$, and $100 \mathrm{pc}$. The CO line luminosity is primarily a

function of $\mathrm{CO}$ mass and density in these models.

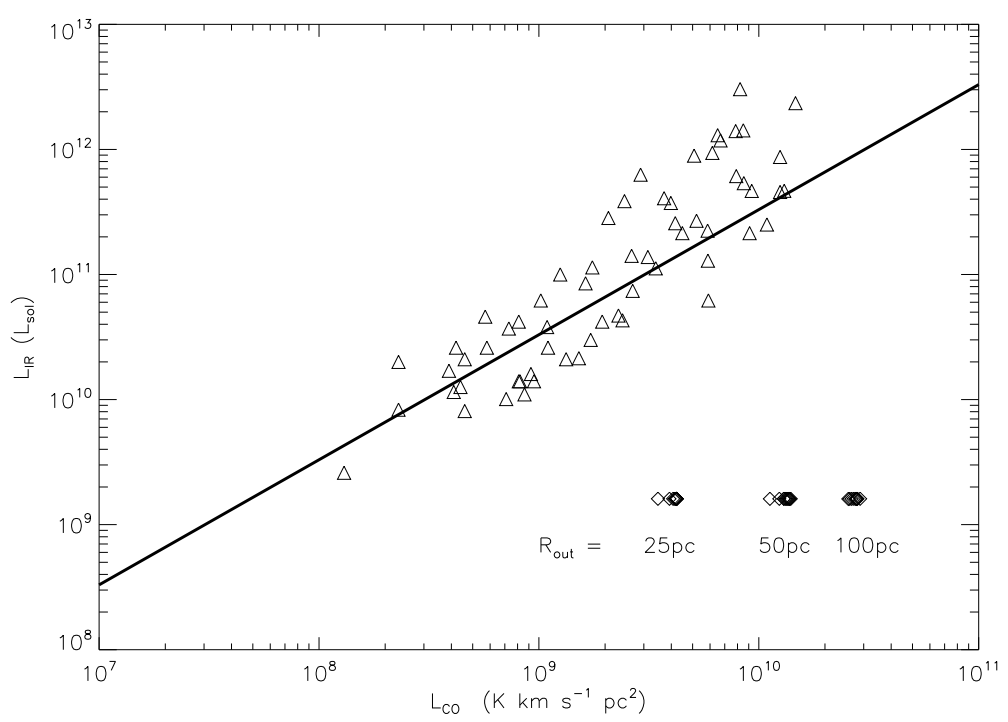

FiguRE 3.3: The embedded SSC models are compared to galaxy data from Gao \& Solomon [65]. The models are two to three orders of magnitude too bright in CO luminosity; if this were an observationally-supported trait of embedded SSCs, then they would outshine their host galaxies. 


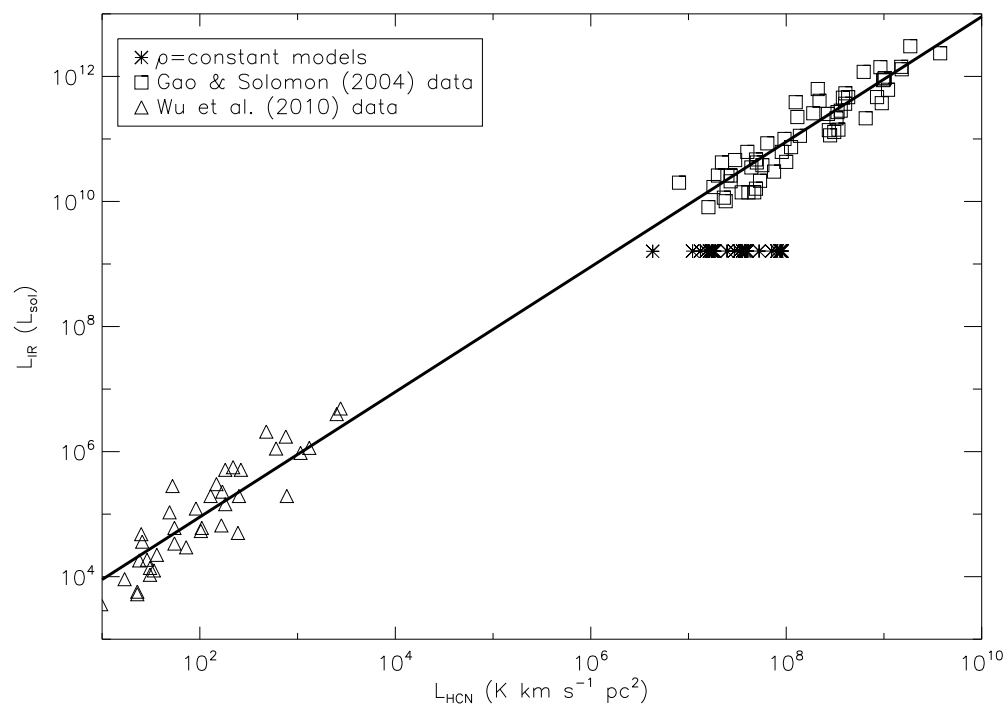

FiguRE 3.4: The embedded SSC models are compared to galaxy data from Gao \& Solomon [65] and Wu et al. [230]. The models are one to three orders of magnitude too bright in HCN luminosity.

envelope at high density near the center and therefore at high optical depth. This geometry effectively shields a percentage of the $\mathrm{CO}$ from the outside, thereby decreasing the observed CO luminosity. This effect is seen in Figure 3.5, in which a constant density envelope's line profile is compared to that for an envelope that is allowed to fall off as $1 / \mathrm{r}$.

The result of the line luminosity's clear dependence on the density profile of the envelope means that the density exponent must be considered in the models. The dependence on density profile plus the superluminal model line luminosities are the primary motivations for re-evaluating the envelope geometry. 


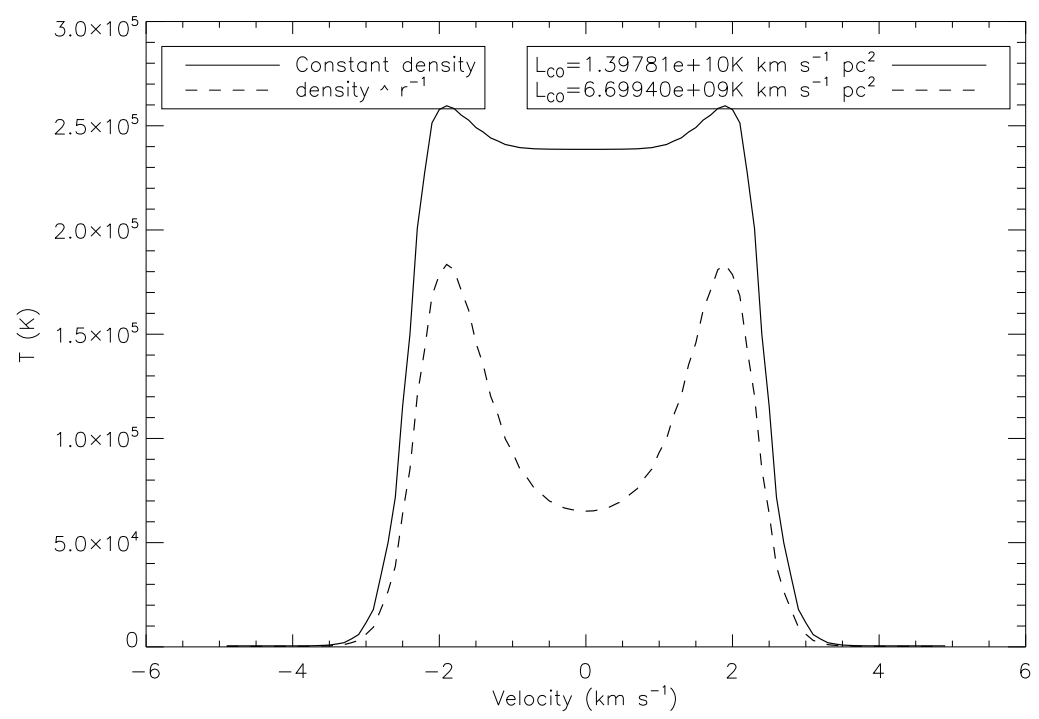

Figure 3.5: The simulated line profile of two embedding envelopes are shown, one with a constant density and one whose density falls off from the center by $1 / \mathrm{r}$. The constant density envelope exhibits a CO line luminosity roughly twice that of its variable model. All other parameters were fixed for this test. 


\subsection{A New Parametrization: New Envelope Ge-}

\section{ometries and Better Agreement with Expec-}

\section{tations}

For the purposes of these models, the star formation efficiency (SFE) is defined as in Ashman \& Zepf [4]:

$$
S F E=\frac{M_{c l}}{M_{e n v}+M_{c l}} .
$$

$M_{c l}$ is the star cluster mass (in stars), and $M_{e n v}$ is the embedding envelope's mass.

Furthermore, we are assuming that the density profile in the envelope is a simple power law:

$$
\rho(r)=C \cdot r^{-\alpha}
$$

The envelope mass can be determined by integrating the density over the volume:

$$
M_{\text {env }}=\int_{R_{\text {inner }}}^{R_{\text {outer }}} \rho(r) \cdot 4 \pi r^{2} d r=\int_{R_{\text {inner }}}^{R_{\text {outer }}} C \cdot r^{-\alpha} \cdot 4 \pi r^{2} d r
$$

or: 


$$
M_{\text {env }}=\frac{4 \pi C}{3-\alpha}\left(R_{\text {outer }}^{3-\alpha}-R_{\text {inner }}^{3-\alpha}\right) .
$$

For a given inner radius, the envelope mass is a function of two unknowns: $\mathrm{R}_{\text {outer }}$ and $\mathrm{C}$. To define $\mathrm{R}_{\text {outer }}$, we define the end of the envelope as where the envelope density has dropped down to the density of the interstellar medium. Presuming that the GMC out of which the SSC is born is embedded in an Hi cloud, then a standard hydrogen density of $30 \mathrm{~cm}^{-3}$ can be assumed [49, Chapter 1, Table 1.3]. We can then solve for $R_{\text {outer }}$ with the prescribed density law:

$$
\begin{gathered}
n\left(R_{\text {outer }}\right)=30 \mathrm{~cm}^{-3}=\frac{C}{m_{p}} \cdot R_{\text {outer }}^{-\alpha} \\
R_{\text {outer }}=\left(\frac{C}{30 m_{p}}\right)^{1 / \alpha}
\end{gathered}
$$

where $m_{p}$ is the mass of a proton, the most abundant particle in the ISM. We may similarly find an expression for $\mathrm{C}$ by solving the density law for $\mathrm{R}_{\text {inner }}$ :

$$
\begin{gathered}
n\left(R_{\text {inner }}\right)=\frac{C}{m_{p}} \cdot R_{\text {inner }}^{-\alpha} \\
C=m_{p} \cdot n\left(R_{\text {inner }}\right) \cdot R_{\text {inner }}^{\alpha} .
\end{gathered}
$$


$\mathrm{R}_{\text {outer }}$ can then be written as a function of $\mathrm{R}_{\text {inner }}$ :

$$
R_{\text {outer }}=R_{\text {inner }} \cdot\left(\frac{n\left(R_{\text {inner }}\right.}{30 \mathrm{~cm}^{-3}}\right)^{1 / \alpha}
$$

and the envelope mass can be written as a function of $\mathrm{n}\left(\mathrm{R}_{\text {inner }}\right), \mathrm{R}_{\text {inner }}$, and $\alpha$ :

$$
M_{\text {env }}=\frac{4 \pi}{3-\alpha} m_{p} n\left(R_{\text {inner }}\right) R_{\text {inner }}^{\alpha}\left(\left[R_{\text {inner }} \cdot\left(\frac{n\left(R_{\text {inner }}\right)}{30 c m^{-3}}\right)^{1 / \alpha}\right]^{3-\alpha}-R_{\text {inner }}^{3-\alpha}\right) .
$$

When $\alpha=0$, then an outer radius must also be defined based on the desired SFE. Based on the analysis given in the Introduction, plus observational constraints [e.g. 138], the SFE is likely to be in the range 5-50\%. Values for $\alpha$ of $1,1.5$, and 2 are modeled for, being the acceptable range of known values for collapsing, lower-mass GMCs [185], and His region densities $\left(\mathrm{n}\left(\mathrm{R}_{\text {inner }}\right)\right.$ in the range $5 \times 10^{3}$ $2 \times 10^{4} \mathrm{~cm}^{-3}$ have been modeled, being an observed range of densities based on thermal radio measurements of embedded SSC environments [9, 99-101, 108, 210]. For the various input parameters, the cloud structure was computed with CLOUDY $[1,62]$. For a 1 Myr old starburst computed with the stellar synthesis code Starburst99 [120], CLOUDY determines the ionization structure of the cloud and then determines the abundances of molecules within its chemical abundance network. The results for a typical simulation for Hydrogen and Carbon are shown in Figure 3.6. For Hydrogen, the ionization front is where the $\mathrm{H}^{+}$and $\mathrm{H}^{0}$ lines cross. 
The UV radiation is not yet insubstantial until past the dissociation front, where the molecular Hydrogen $\left(\mathrm{H}_{2}\right)$ becomes more abundant than $\mathrm{H}^{0}$ by number. The ionization structure of Carbon reveals that the HiI region contains more heavily ionized carbon close to the source, and that CIII is the most abundant carbon ion at the ionization front. CiI dominates outside the ionization front well past the hydrogen dissociation front, and molecules do not begin to form until well into the molecular gas, when temperatures have gotten down to $\sim 50 \mathrm{~K}$ and less.

Using the molecular abundances produced by CLOUDY, we then ran RATRAN models for the range of input parameters to determine the CO $(J=1-0)$ line luminosity and dust radiative transfer models (using the code from Whelan et al. [218]) to determine infrared luminosities (1-1000). The results are shown in Figure 3.7. Three different cluster masses were computed for: $\mathrm{M}_{c l}=10^{4}, 10^{5}$, and $10^{6} \mathrm{M}_{\odot}$. For the constant density models exhibited in the upper left panel, $\mathrm{SFE}=10 \%$ was assumed so that the envelope mass was locked. The spread, therefore, is a function of the temperature structure in the cloud for $\mathrm{R}_{\text {inner }}$ values between 0.1 and $5 \mathrm{pc}$. For the other models, the spread is largely a function of mass and line optical depth: the more massive clouds have higher CO luminosities, and they tend to cluster towards the high range of luminosity because the cloud thickness eventually makes the CO line optically thick. There is no discernible spread in infrared luminosity for any of the models because these envelope geometries are necessarily optically thick to UV light (they are models of embedded SSC environments) so the re-processed light from the dust emission remains constant despite changes in 

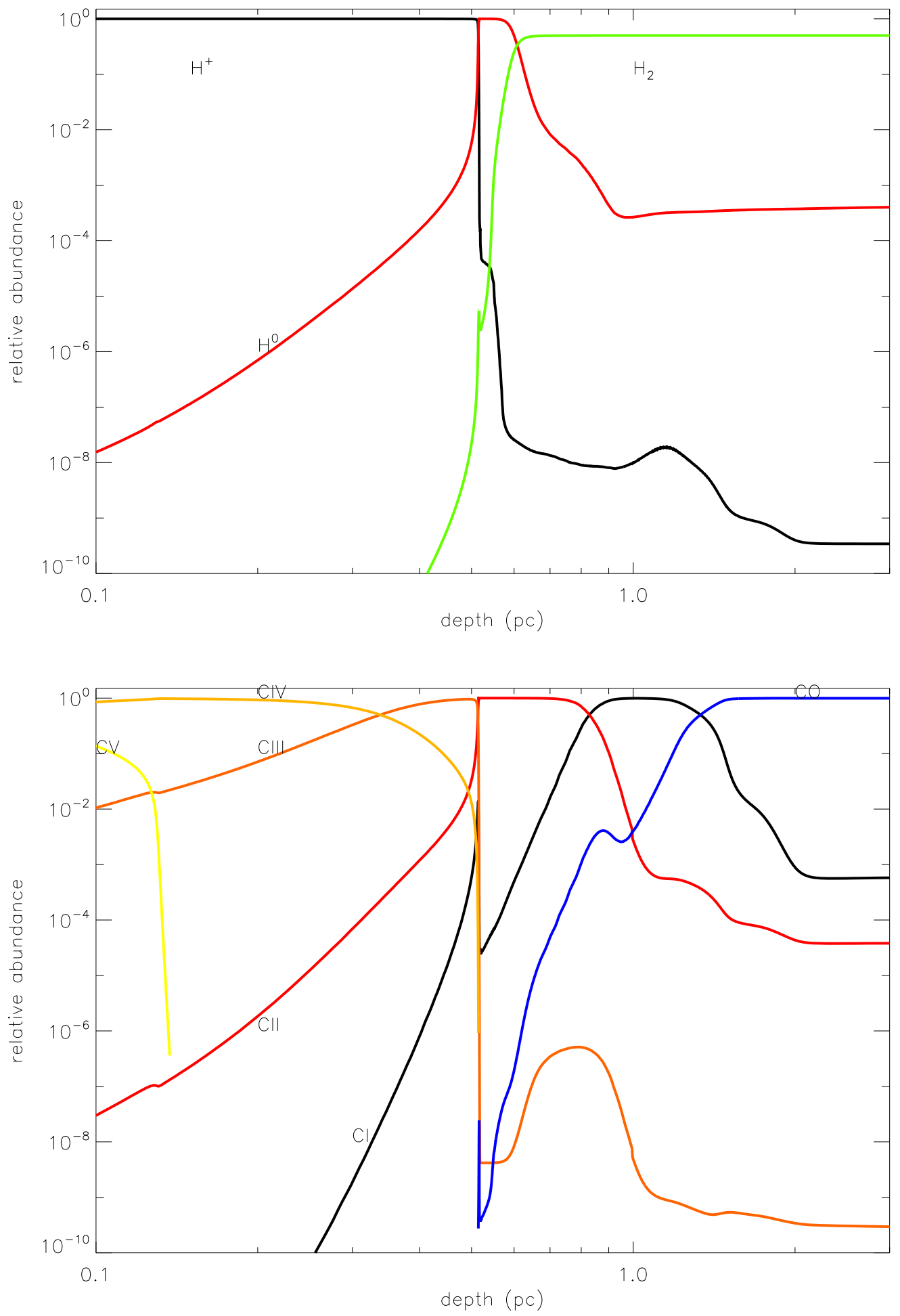

FiguRE 3.6: The Hydrogen and Carbon ionization structure and relative abundances of species through a molecular cloud, plotted at a distance from the central source. 


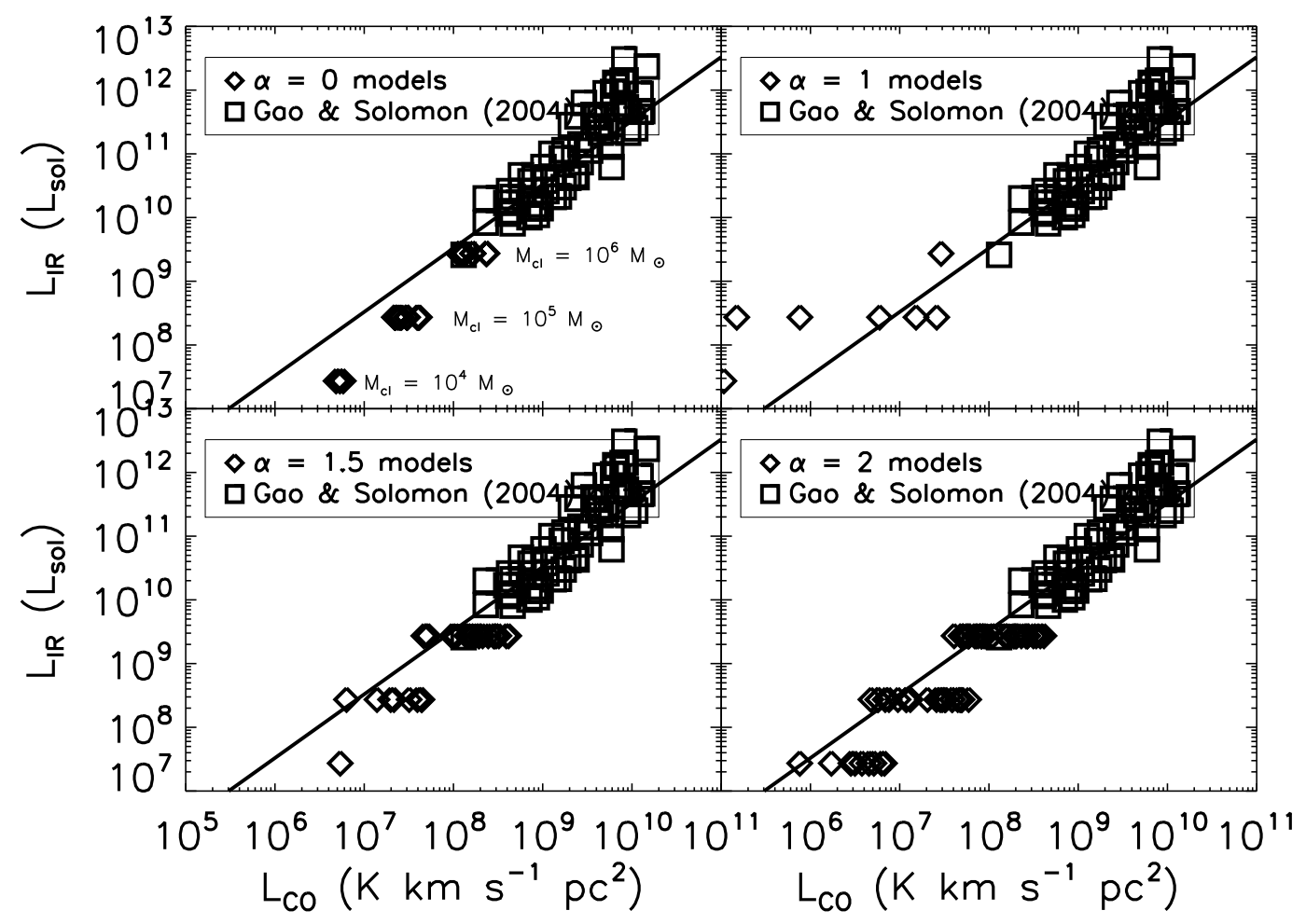

Figure 3.7: The $\mathrm{L}_{I R}$ is plotted versus $\mathrm{L}_{C O}$ for the models where $\mathrm{M}_{\text {env }}\left(\mathrm{R}_{\text {inner }}\right.$, $\mathrm{n}\left(\mathrm{R}_{\text {inner }}, \alpha\right)$. For the $\alpha=0$ models, a $\mathrm{SFE}=10 \%$ was assumed, and the requisite small spread is due to the temperature structure within the cloud. For the other $\alpha$ values, the spread is a function of cloud mass (or SFE) and optical depth.

model geometry.

Despite the spread in line luminosity seen, and the spread's known dependence on envelope mass and line optical depth, these results are very inconclusive: for the different density power laws between 0 and -2 , the results overlap. And without a model for the rotation and effects of turbulence on the line profile, the line profiles cannot yet be used to discern between the different power law values.

There is the additional problem that, these models being one-dimensional, clumping, either due to interstellar turbulence or else gas falling along filaments, is a likely feature of these envelopes; it was shown that clumping can be used to explain 
light leakage from embedded SSC environments [218]. While the effect of clumping on molecular line emission is as yet unknown, it seems likely that clumping could be modeled to produce at least observable effects: (1) clumping will produce areas of low- and high-density that will vary the molecular line optical depth along the line of sight - this should be a minor effect for high-density tracers (e.g. $\mathrm{HCN},{ }^{13} \mathrm{CO}$ ), but may be major for low-density tracers (e.g. CO) in a way that is analogous to results involving dust [218]; (2) Clumping will have an effect on the line luminosity due to density dependence - because a large percentage of the molecular mass will lie in the high density clumps (i.e. higher than the average cloud density) and not the diffuse interclump medium where it would be exposed to the UV field from the central source, the line luminosity will be altered because of the higher collisional rates in the clumps.

\subsection{Conclusions and Future Directions}

We have determined $\mathrm{CO}$ and $\mathrm{HCN}$ line luminosities and infrared luminosities for embedded SSC envelopes and compared those results to a sample of galaxies from Gao \& Solomon [65] for which a power law correlation was determined over two orders of magnitude. The model geometries are parametrized as a function of inner radius, gas density at the inner radius, and envelope density power low. We employed a photodissociative chemistry code and one-dimensional dust and molecular line radiative transfer in order to determine the correct abundances 
and emission from realistic envelope geometries. The results generally follow the expected power law correlation found in Gao \& Solomon [65], but the model parameter space studied showed significant degeneracies between the different power law density profile results. This, plus the consideration that clumpiness has not yet been taken into account, require a significant re-examination of this problem, including three-dimensional that will allow us to compute the effects of clumping acurately, and also likely hydrodynamical simulations that will produce realistic clumping geometries.

Much work is being done on making three-dimensional codes available for molecular ISM studies. 3D PDR is a new code that solves a photodissociative chemical network for arbitrary 3D geometries [14]. The chemical network available for this code is relatively small, however, and while it will compute molecular abundances for most of the commonly observed molecules it will not be asaccurate as CLOUDY. Also, it assumes plane-parallel geometry which greatly restricts its use for super star clusters: a central source surrounded by clumpy gas needs to be illuminated spherically so that light propagation through multiple clumps of gas is performed correctly. Molecular line radiative transfer has also become generalized with the creation of LIME [19], the 3D version of Ratran.

With these new codes, it may be possible to begin using 3D codes to determine precise molecular cloud properties in SSC environments. This field of study will also benefit greatly with long-baseline studies of embedded SSC environments, which will be able to observe individual embedded SSC environments in isolation 
Chapter 3. First Models of the Molecular Emission from Embedded Super Star Clusters

and, in some cases, at resolutions that will provide spatial information of the cloud's emission. 


\title{
Chapter 4
}

\section{An In-Depth View of the}

\section{Mid-Infrared Properties of Point}

\author{
Sources and the Diffuse ISM in
}

\section{the SMC Giant HII Region, N66}

\subsection{Abstract}

The focus of this work is to study mid-infrared point sources and the diffuse interstellar medium $(\mathrm{ISM})$ in the low-metallicity $\left(\sim 0.2 \mathrm{Z}_{\odot}\right)$ giant HII region N66 in order to determine properties that may shed light on star formation in these conditions. Using the Spitzer Space Telescope's Infrared Spectrograph, we study 
polycyclic aromatic hydrocarbon $(\mathrm{PAH})$, dust continuum, silicate, and ionic line emission from 14 targeted infrared point sources as well as spectra of the diffuse ISM that is representative of both the photodissociation regions (PDRs) and the HiI regions. Among the point source spectra, we spectroscopically confirm that the brightest mid-infrared point source is a massive embedded young stellar object, we detect silicates in emission associated with two young stellar clusters, and we see spectral features of a known Be star that are commonly associated with Herbig Be stars. In the diffuse ISM, we provide additional evidence that the very small grain population is being photodestroyed in the hard radiation field. The $11.3 \mu \mathrm{m}$ PAH complex emission exhibits an unexplained centroid shift in both the point source and ISM spectra that should be investigated at higher signal-to-noise and resolution. Unlike studies of other regions, the $6.2 \mu \mathrm{m}$ and $7.7 \mu \mathrm{m}$ band fluxes are decoupled; the data points cover a large range of $\mathrm{I}_{7.7} / \mathrm{I}_{11.3} \mathrm{PAH}$ ratio values within a narrow band of $\mathrm{I}_{6.2} / \mathrm{I}_{11.3}$ ratio values. Furthermore, there is a spread in PAH ionization, being more neutral in the dense PDR where the radiation field is relatively soft, but ionized in the diffuse ISM/PDR. By contrast, the PAH size distribution appears to be independent of local ionization state. Important to unresolved studies of extragalactic low-metallicity star-forming regions, we find that emission from the infrared-bright point sources accounts for only 20-35\% of the PAH emission from the entire region. These results make a comparative dataset to other star-forming regions with similarly hard and strong radiation fields. 


\subsection{INTRODUCTION}

The Small Magellanic Cloud (SMC) is an excellent test-bed for studying star formation in a low-metallicity environment. Its low metallicity $\left[\sim 0.2 \mathrm{Z}_{\odot}\right.$ determined from numerous elemental abundances; 168] and strong interstellar radiation field [ISRF; 4-10 $\left.\mathrm{G}_{0}, 40\right]$ make it an important contrasting environment to star forming environments in the Milky Way or the Large Magellanic Cloud (LMC). The SMC is also a good comparative theater to studies of 'passive' star formation in blue compact dwarf galaxies [BCDs; see 204, for the distinction between "active" and "passive"], because their star-forming regions have similar densities $\left(\sim 100 \mathrm{~cm}^{-3}\right)$, star formation rates $\left(\sim 0.1 \mathrm{M}_{\odot} y r^{-1}\right)$, radiation field hardnesses, and the SMC is the lowest-metallicity nearby star-forming region [126, 222].

N66 [73] is the largest HII region in the SMC, covering an area on the sky of approximately $180 " \times 300 "$, and therefore offers the best view of large-scale star formation in the SMC. It surrounds a large stellar association known as NGC 346. N66 contains $33 \mathrm{O}$ stars distributed across the HiI region, which is about half the number for the entire SMC, and 11 of them are earlier than type O7 [128]. The most massive star is of O3III $\left(\mathrm{f}^{*}\right)\left(\sim 100 \mathrm{M}_{\odot}\right)$ or O3vf* $\left(\sim 90 \mathrm{M}_{\odot}\right)$ type $[130,215]$. The O stars illuminate the surrounding ISM and are responsible for an $\mathrm{H} \alpha$ luminosity of about 60 times that of the Orion nebula [104]. UV and optical spectra have been used to derive an age of about 3 Myr for the O stars in N66 and a metallicity of $0.2 \mathrm{Z}_{\odot}$ [the metallicity has been determined independently 
for individual $\mathrm{O}$ stars, forbidden line emission originating in the gas, and spectral models; 17, 70, 115].

N66 is experiencing ongoing star formation. Simon et al. [189] identified about 100 embedded YSOs with Spitzer IRAC and MIPS photometry, and Gouliermis et al. [67] found a further 263 candidate young stellar sources including intermediate mass pre-main sequence and Herbig AeBe stars, as well as massive YSO candidates. The first mid-IR study of N66, with ISOCAM, showed strong nebular [SIV] $10.51 \mu \mathrm{m}$ and [NeIII] $15.56 \mu \mathrm{m}$ emission across the region that is indicative of young and massive (O- and B-type) stars, the presence of faint polycyclic aromatic hydrocarbon $(\mathrm{PAH})$ emission bands, a mid-infrared continuum from very small grain (VSGs) and large thermal dust grain emission, and an ISRF at $1600 \AA \geq 10^{5}$ times that of solar [38]. A companion paper to Contursi et al., Rubio et al. [167], included [OIII] $\lambda 5007, \mathrm{H}_{2} \mathrm{v}(1-0) \mathrm{S}(1) 2.12 \mu \mathrm{m}$, and $\mathrm{CO}$ observations to show that the peaks in $\mathrm{H}_{2}, \mathrm{CO}$, and $\mathrm{PAH}$ emission are all spatially correlated across the photodissociation regions (PDRs) in general, and further suggested that the $\mathrm{CO}$ has been largely photodissociated across the His region by the O star population, and exists only in small clumps. Sandstrom et al. [174] included N66 as part of a study of PAHs in HII regions across the SMC, and determined that the PAH population is both smaller and less ionized than in higher-metallicity galaxies. In two comparison studies, the atomic/ionic gas content and the effects of metallicity on PAH emission were studied for N66, 30 Doradus in the LMC, and NGC 3603 in the Milky Way $[115,117]$. The elemental abundances were determined for each 
region using the ionic forbidden lines from mid-infrared spectra; for N66, the results confirmed that the metallicity is about $0.2 \mathrm{Z}_{\odot}$. It was discovered that the PAHs are photodestroyed in radiation fields where nebular $[\mathrm{NeIII}] /[\mathrm{NeII}] \gtrsim 3$, and that this photodestruction law is independent of metallicity. What is still unclear about N66 is where the PAH emission originates, and what conditions are traced by the PAH emission. In particular, PAH ionization state, which is a function of the ultraviolet (UV) radiation field, is also equally sensitive to electron density [charge state $\mathrm{Z} \propto \mathrm{G}_{0} \mathrm{~T}^{1 / 2} / n_{e} ; 207$, section 6.3.8], and there are cases evident in the literature that suggest that neutral PAHs have the ability to form inside HII regions [e.g. in the vicinity of the Horsehead Nebula: 36].

The reason that star formation is often traced by emission from PAHs [150] is that, while PAHs are sensitive to excitation from a broad range of wavelengths (UV-IR), they are particularly susceptible to excitation by UV photons. PAH emission is commonly observed in the photodissociation regions (PDRs) around young massive clusters $[119,206]$. These spectral features are predominantly present from 3-17 $\mu \mathrm{m}$. The molecules responsible for this emission are typically dozens to thousands of carbon atoms large. Following photoexcitation, they emit by fluorescence from stretching and bending modes either from the carbon-to-hydrogen $(\mathrm{C}-\mathrm{H})$ or carbon-to-carbon (C-C) bonds.

Due to the stochastic excitation and emission mechanism as well as the ionization balance of PAHs, the local physical conditions have a large impact on the observed $\mathrm{PAH}$ band ratios via radiation field hardness, column density, dust temperature, 
and dust composition $[80,149]$. Due to the relatively low ionization potentials of PAHs [about 6-8 eV for small PAHs; 207, Table 6.1], PDRs are expected to be dominated by ionized PAHs whereas regions with weaker radiation fields, such as the diffuse ISM in the Milky Way or inside molecular clouds, should have largely neutral or negatively-charged PAHs [8].

In order to study the mid-infrared properties of N66 in greater detail with particular emphasis on the $\mathrm{PAH}$ emission as an independent tracer of the physical conditions across the region, we present Spitzer Space Telescope/IRS spectra of a number of infrared point sources and use the spectral information along the entire IRS long slits to study the dust and gas emission throughout the Hir region and photodissociation region (PDR). We present the observations in Section 4.3, describe our data reduction in Section 4.4, present our analysis in Section 4.5, and summarize the results in Section 4.6.

\subsection{OBSERVATIONS}

N66 (Figure 4.1) was observed as part of the IRS GTO program to study massive star formation in giant HII regions in the Local Group (PID 63). Imaging using Spitzer/IRAC [58] of the whole region revealed a number of bright point sources, the brightest eight of which were chosen for spectroscopic follow-up with the low- and high-resolution modules of the Spitzer Infrared Spectrograph [IRS; 
81]. The present study concentrates on the short-wavelength low-resolution spectra only $(\lambda<14.7 \mu \mathrm{m})$; the high-resolution Spitzer spectra have been analysed in Lebouteiller et al. $[115,117]$ to determine element abundances and to constrain the strength of the interstellar radiation field (ISRF) using forbidden emission lines, and the long-wavelngth low-resolution data $(15 \mu \mathrm{m}<\lambda<37 \mu \mathrm{m})$ have not been included due to the spatial resolution and slit orientation mismatch. This dataset is therefore in contrast to that presented in Sandstrom et al. [174], in which longand short-wavelength spectral maps are used to analyze N66. While they analyzed full spectral maps of the entire region, the data we present here is deeper and allows us to study the faint extended emission around the point sources at a level of detail that the full maps did not allow.

The pointings for the original eight positions (Astronomy Observing Request 4385024) were systematically offset by about 5 with respect to the actual source positions. As a result, most of the intended point sources were partially or wholly outside of the 3.6-wide slit. However, there is at least one point source in each of these slits regardless, though they are not always the intended ones. In order to target the intended point sources, N66 was re-observed with correct astrometry (AOR 16207872). The point sources detected in the original and re-observed samples are shown in Figure 4.2 along with the slit orientations. See Table 1 for a full list of the point source (PS) positions, labeled in order of decreasing right ascension, and Table 2 for a list of slit centroids.

Three exposures were taken at each nod position in staring mode (see the Spitzer/IRS 


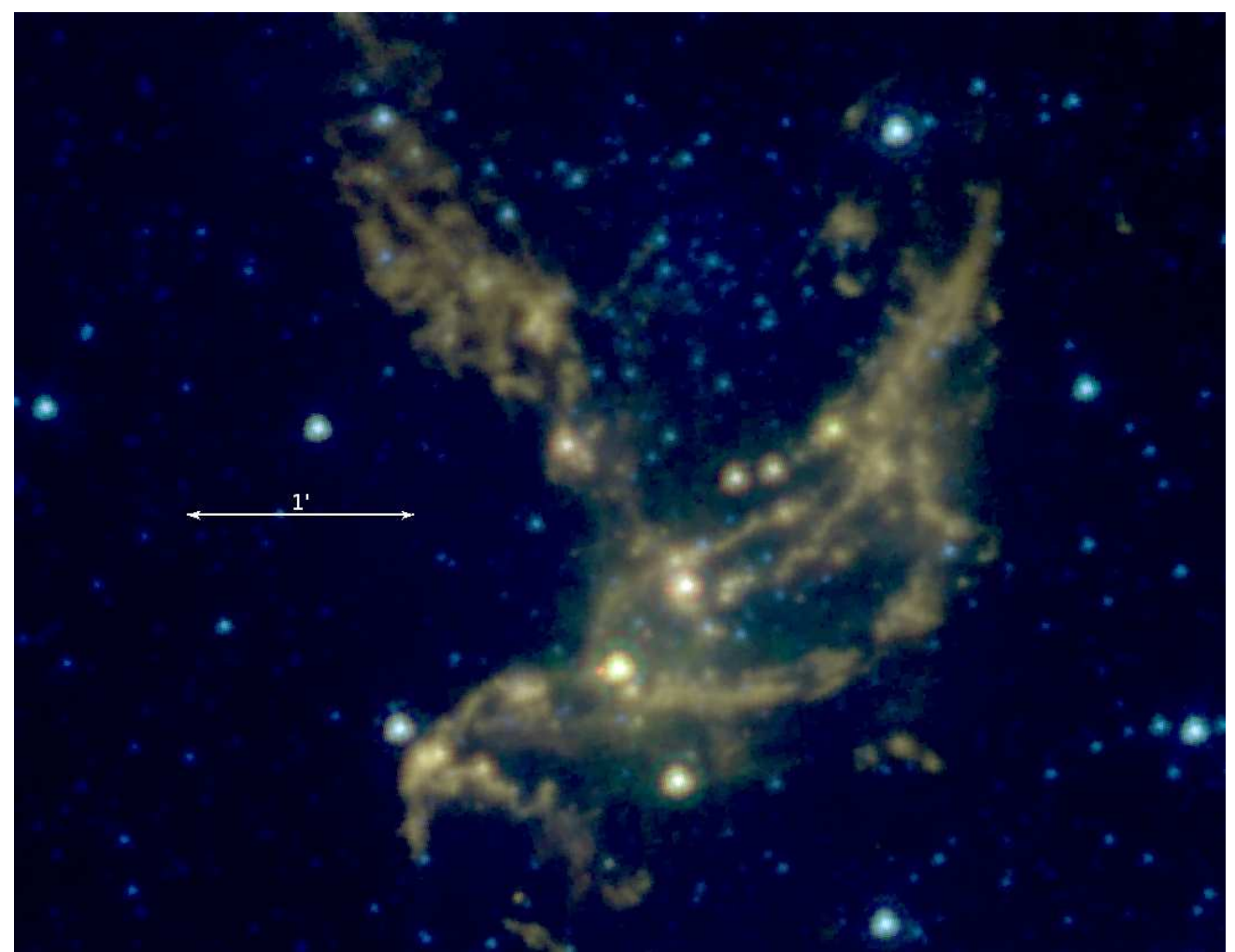

Figure 4.1: A Spitzer/IRAC 3-color image of N66. Blue is $3.6 \mu \mathrm{m}$, green is $5.8 \mu \mathrm{m}$, and red is $8.0 \mu \mathrm{m}$. The $5.8 \mu \mathrm{m}$ and $8.0 \mu \mathrm{m}$ images trace the PAH emission in the region, highlighting the PDRs, while the $3.6 \mu \mathrm{m}$ image traces the stellar population; in particular, the old star cluster, BS 90, is visible to the north of NGC 346. The angular scale of 1 corresponds to a physical distance of $17.6 \mathrm{pc}$ at the distance of NGC 346 .

manual), where the source is positioned at $1 / 3(\operatorname{nod} 1)$ and $2 / 3(\operatorname{nod} 2)$ of the slit length. The original observations had 60-second exposure times, whereas the reobserved sample have 15-second exposure times.

The low-resolution spectra $(\lambda / \Delta \lambda \sim 65$-130) cover the spectral range 5.2-14.7 $\mu \mathrm{m}$ using the short-low (SL) module. The SL-1 slit covers the spectral range 7.4$14.7 \mu \mathrm{m}$ and the SL-2 slit covers the spectral range 5.2-7.7 $\mu \mathrm{m}$. The sizes of the two slits $(3.7 \times 57$ and $3.6 \times 57$ respectively $)$ correspond to a physical size of $1.1 \times 16.7 \mathrm{pc}$ at the distance of N66 [we adopt a value of $60.6 \mathrm{kpc}$ from 77]. 

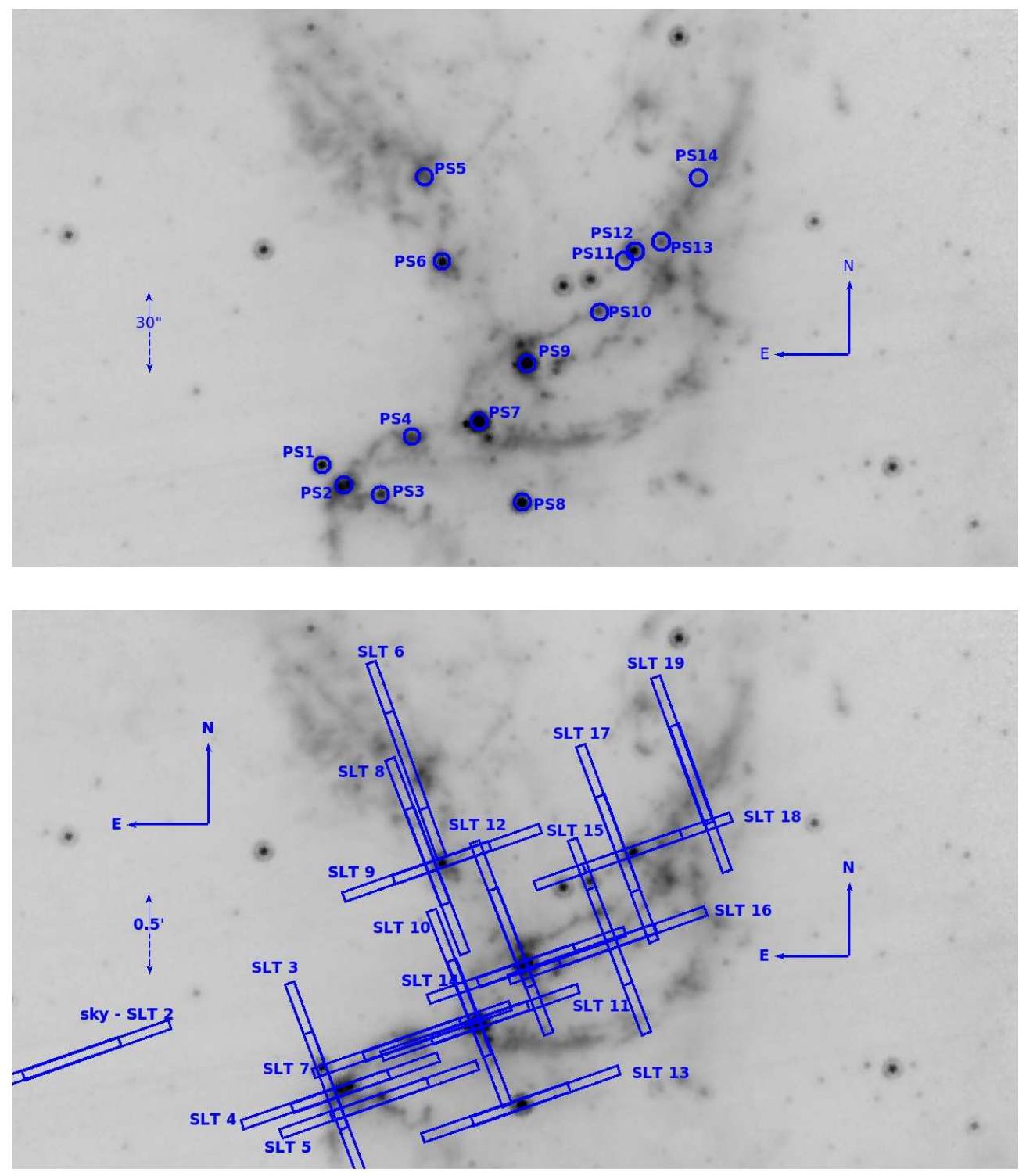

Figure 4.2: The IRAC $8.0 \mu \mathrm{m}$ image with the observed point sources circled and labeled in blue, and the labeled slit positions across the region. See Tables 1 and 2 for coordinate information. 
Therefore the point sources, with a FWHM at $6 \mu \mathrm{m}$ of 4 pixels out of 34 , are resolved down to about 2 pc along the slit.

\subsection{DATA REDUCTION}

\subsubsection{Spitzer/IRS Image Reduction}

The Spitzer/IRS images were processed with the Spitzer Science Center (SSC) pipeline, version S18.7. The basic calibrated data (BCD) were used. Images were cleaned by interpolating values over flagged bad pixels using the $S S C$-provided IDL package IRSCLEAN ${ }^{1}$. After cleaning, the observed sky positions were subtracted from the other positions in their respective AORs to remove any intervening flux from zodiacal light, the Milky Way, and foreground SMC. Since the sky positions are relatively far from N66, we may assume that emission in the sky spectra is primarily from the diffuse SMC and not from N66. The spectra of these sky positions exhibit [SIV] emission but no dust features. The strength of the sky position's [SIV] emission is $7.9 \times 10^{-21} \mathrm{~W} \mathrm{~cm}^{-2}$, which is almost $50 \%$ the value for the lowest [SIV] flux found in the extended emission spectra (see $\S 4.4 .2$ ), and less than $20 \%$ of the flux for the rest of the spectra.

${ }^{1}$ All $\quad S S C$-provided packages may be found
//irsa.ipac.caltech.edu/data/SPITZER/docs/dataanalysistools/




\subsubsection{Spectral Extractions}

Using the optimal extraction routines available in the Spectroscopy Modeling Analysis and Reduction Tool [SMART-AdOpt; 76, 116], point source and extended emission can be simultaneously extracted. SMART's optimal extraction routines fit a polynomial function to the extended emission as well as template supersampled point spread functions (PSFs) to point sources in the slit, as shown in Figure 4.3. The backgrounds are relatively smooth for these spectra, and polynomials of four orders and less were used. Details, including how to fit partially extended point sources and measuring the residuals, are presented in Lebouteiller et al. [116]. However, the fundamental operation demands that for each wavelength element, the relative weights to the total flux assigned to the point source(s) and background are measured simultaneously. Therefore, the flux is distributed to each component in a way that agrees with the combination of point source(s) and polynomially-fitted background.

The advantage of simultaneous point source and extended emission extraction compared to more traditional, variable column extractions is illustrated in Figure 4.4. Two point sources have been extracted using two different methods as an example. The source in the left-hand plot is a massive embedded young stellar object (YSO), PS7, and the right-hand plot shows the spectrum of the unresolved dust emission associated with the young star cluster NGC 346, PS9. The "column extraction' extracts all of the emission inside a column that varies in width with 
the PSF. The 'optimal extraction' uses the SMART-AdOpt routines. There are significant differences revealed by the optimal extraction method. For example, the [SIV] $10.51 \mu \mathrm{m}$ emission line, which is often prominent in HII regions, is not associated with the point source spectra; the reason it appears in the column extraction is because it is nebular emission along the line-of-sight to the point source. In fact, we would get the wrong diagnostic with a variable column extraction, since the nebular $[\mathrm{SIV}]$ would be erroneously assigned to the point source. The [NeII] $12.81 \mu \mathrm{m}$ emission line is also nebular in nature for the massive embedded YSO (left-hand plot). In addition to the nebular forbidden atomic lines, a certain amount of the dust continuum is also missing from the optimally extracted spectra, meaning that it is also nebular in nature. By separating out the point source from the line-of-sight nebular emission, we have separate views of emission that is associated with the unresolved point sources in N66 and emission that originates in the diffuse HII region and PDR.

Optimal extraction of all of the pointings was performed, producing point source and extended emission spectra from the full slit length for each pointing. Two of the re-observed pointings have extended emission spectra that were dominated by noise and are not used in the analysis.

Observations where the point source lies partially outside of the slit in the dispersion direction were extracted with a special tool developed for and included in SMART-AdOpt. Applying the centered PSF template to an observation with 


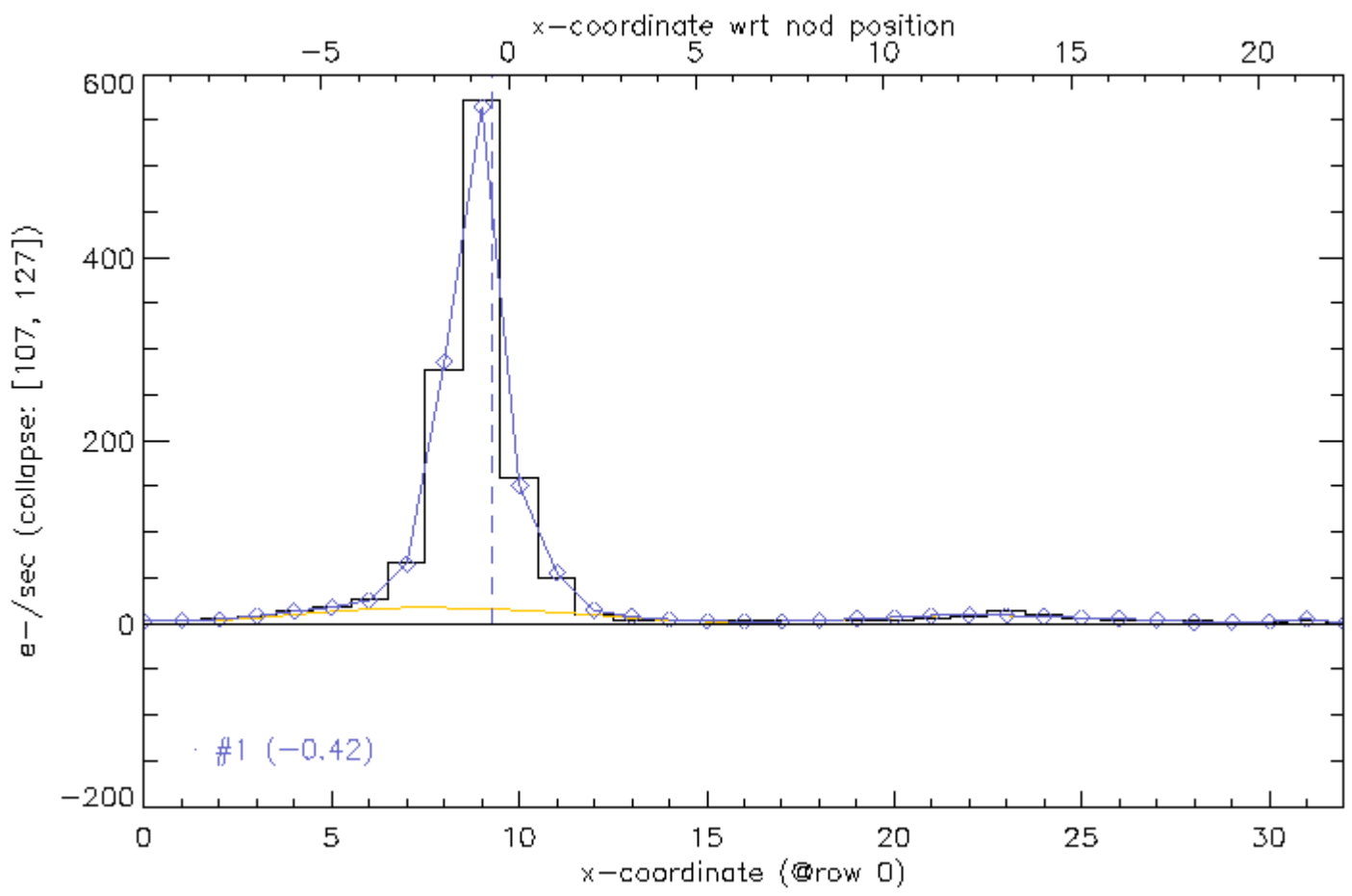

FIgURE 4.3: The spatial extent of an extracted point source and the underlying extended emission for the 7-9 $\mu \mathrm{m}$ continuum as output by SMART-AdOpt. The yellow line is a polynomial fit to the background emission in the slit and a stellar template is used to fit the point source emission. The IRS data are shown in black histogram. The point source is labeled, as would other point sources found, at the bottom of the plot, along with its centroid in pixels with respect to the nod position (on top of the plot) and with respect to the edge of the slit

(bottom of the plot).

incorrect pointing makes the spectral shape incorrect, but accounting for the pointing error allows a properly-shaped PSF to be fit to the point source so that regular optimal extraction and accurate flux calibration can be done. For details on the source extraction for sources offset in the dispersion direction, see Lebouteiller et al. [116]. The reduced spectra are shown in Appendix 1 at the end of this paper. 


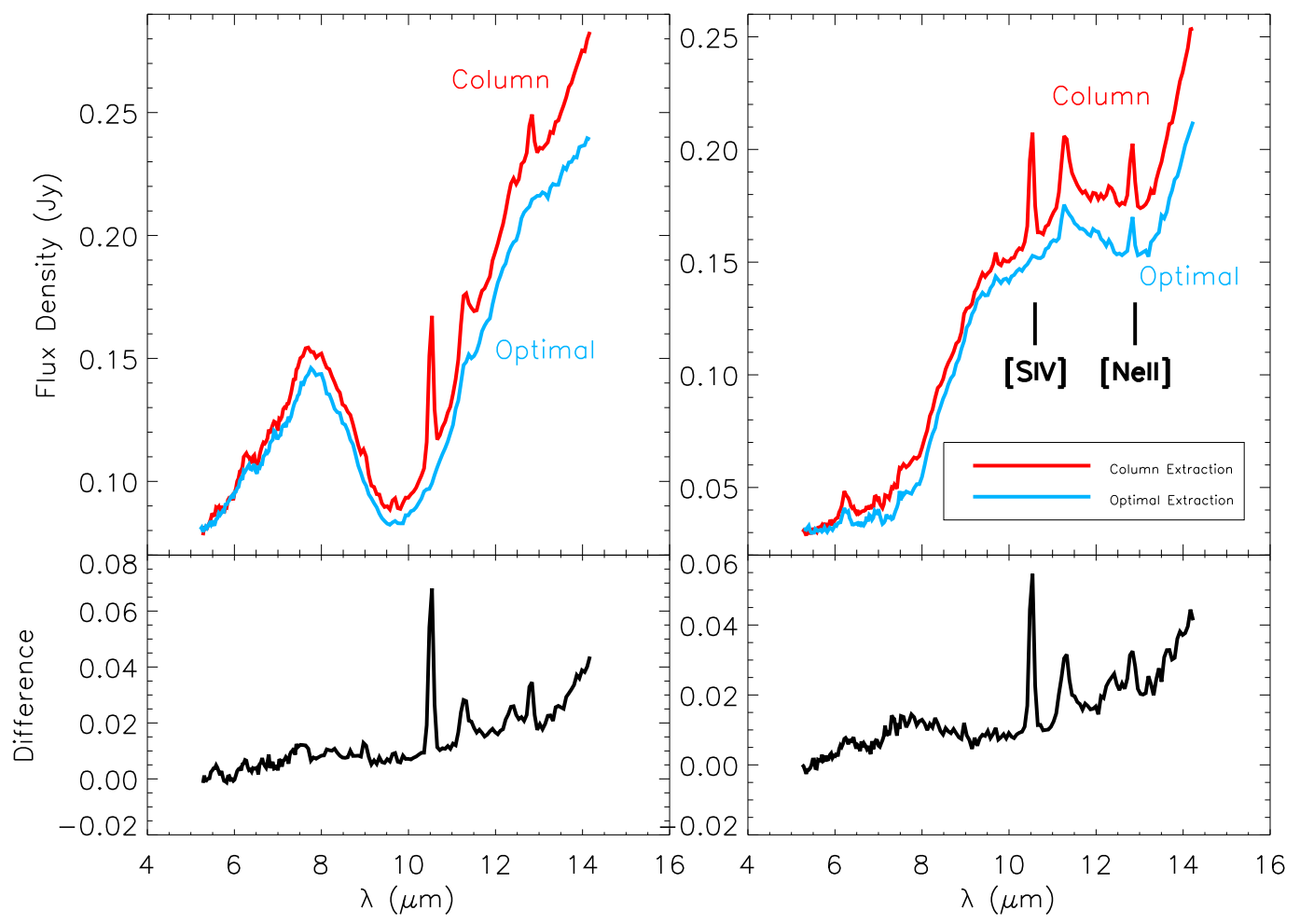

Figure 4.4: Variable column extractions (in red) are shown overplotted with optimal point source extractions (in blue) for the two brightest IR point sources in N66: the massive YSO in N66A (left, PS7) and NGC 346 (right, PS9). The column extractions include a significant amount of emission along the line-ofsight that is not associated with the point source: the [SIV] $10.51 \mu \mathrm{m}$ emission, a portion of the [NeII] $12.81 \mu \mathrm{m}$ emission, and some dust continuum emission is not associated with these point source spectra, but is nebular in nature. This nebular emission is shown in the "Difference" spectra in the bottom panels.

\subsubsection{Spectral Decomposition}

Typical mid-infrared spectra of HII regions and their surrounding PDRs have the following features: a dust continuum, several prominent PAH features, molecular hydrogen emission lines, atomic and ionic emission lines, and absorption or emission features due to small silicaceous grains centered at $9.8 \mu \mathrm{m}$ and $17 \mu \mathrm{m}$. In order to study the dust and gas diagnostics in N66, all of the spectra were decomposed into these components. 
We used the specral decomposition tool PAHFIT [193]. PAHFIT models the dust continuum that underlies the PAH features with as many as eight blackbody continua at fixed temperatures less than $300 \mathrm{~K}$, fits the silicate absorption features at $9.8 \mu \mathrm{m}$ and $17 \mu \mathrm{m}$, and models the PAH features with asymmetric Drude profiles. In order to fit the spectra for N66 with PAHFIT, a couple of small changes were made. In some cases, the input full-width at half maxima (FWHM) of the $6.2 \mu \mathrm{m}$ and $11.3 \mu \mathrm{m}$ features needed to be changed in order to fit the features more precisely ( see $\S 4.5 .3$ below for a discussion about the $11.3 \mu \mathrm{m}$ PAH feature profiles). Second, for sources that exhibit a silicate emission feature, two additional Drude profiles were added to model the $9.8 \mu \mathrm{m}$ silicate emission feature: these Drude profiles, centered at $9.2 \mu \mathrm{m}$ and $10.0 \mu \mathrm{m}$, have FWHM of $0.3 \mu \mathrm{m}$ and $0.2 \mu \mathrm{m}$ respectively. For spectra with silicate emission features included, PAHFIT did not also fit silicate absorption features. Due to the presence of Hydrogen recombination lines in some of the spectra, we include the Hi 6-5 $7.46 \mu \mathrm{m}$ line to PAHFIT's line list. Example point source and extended emission PAHFIT fits are shown in the top panels of Figure 4.5, where the plotting scheme is explained in the caption. One major difference in the fits between the point source and extended emission spectra is that the silicate absorption feature is only fit to those point source spectra that do not explicitly require a silicate emission feature, and none of the extended emission features exhibit silicate absorption in the final fits.

There is some concern that PAH fitting routines like PAHFIT may be introducing 
errors unconsciously into the PAH fluxes. When applying PAHFIT, the underlying PAH "plateaux" are incorporated into the wings of the Drude profiles used to fit the PAH bands, in particular for the $7.7 \mu \mathrm{m}$ band. Peeters et al. (2013, in prep.=aration) has found that the spatial morphology of the plateau is quite distinct from that of the $6.2,7.7$, and $8.6 \mu \mathrm{m}$ PAH bands in data of the reflection nebula NGC 2023. To see if the PAH plateaux in the N66 spectra is also decoupled from the PAH features, we have fit a spline to the underlying continuum and measured the PAH features above the continuum, as in Galliano et al. [64]. He have determined that, although the exact values for the PAH band fluxes differ depending on the decomposition method used, the trends discussed in detail in $\S$ 4.5.5 hold regardless. The spectral decomposition method illustrated in Figure 4.5 is a reliable method for comparing PAH band fluxes.

Due to concerns about error propagation from extraction to decomposition, the errors of the PAH feature strengths were determined with a Monte Carlo method. The root mean square error in the continuum near $6 \mu \mathrm{m}$ was measured for each spectrum and then used to randomly perturb the spectrum. $6 \mu \mathrm{m}$ was chosen because the noise is systematically higher at the shortest wavelengths in the SL spectrum, due to the lower signal in these sources at this wavelength. The noise is, on average, two times greater at $6 \mu \mathrm{m}$ than it is at $14 \mu \mathrm{m}$, measured as the standard deviation of the root mean square of the difference of the unperturbed spectrum and the best fit PAHFIT model. The spectrum, including the random noise, was then fit with PAHFIT and the resultant strengths of the PAH features 

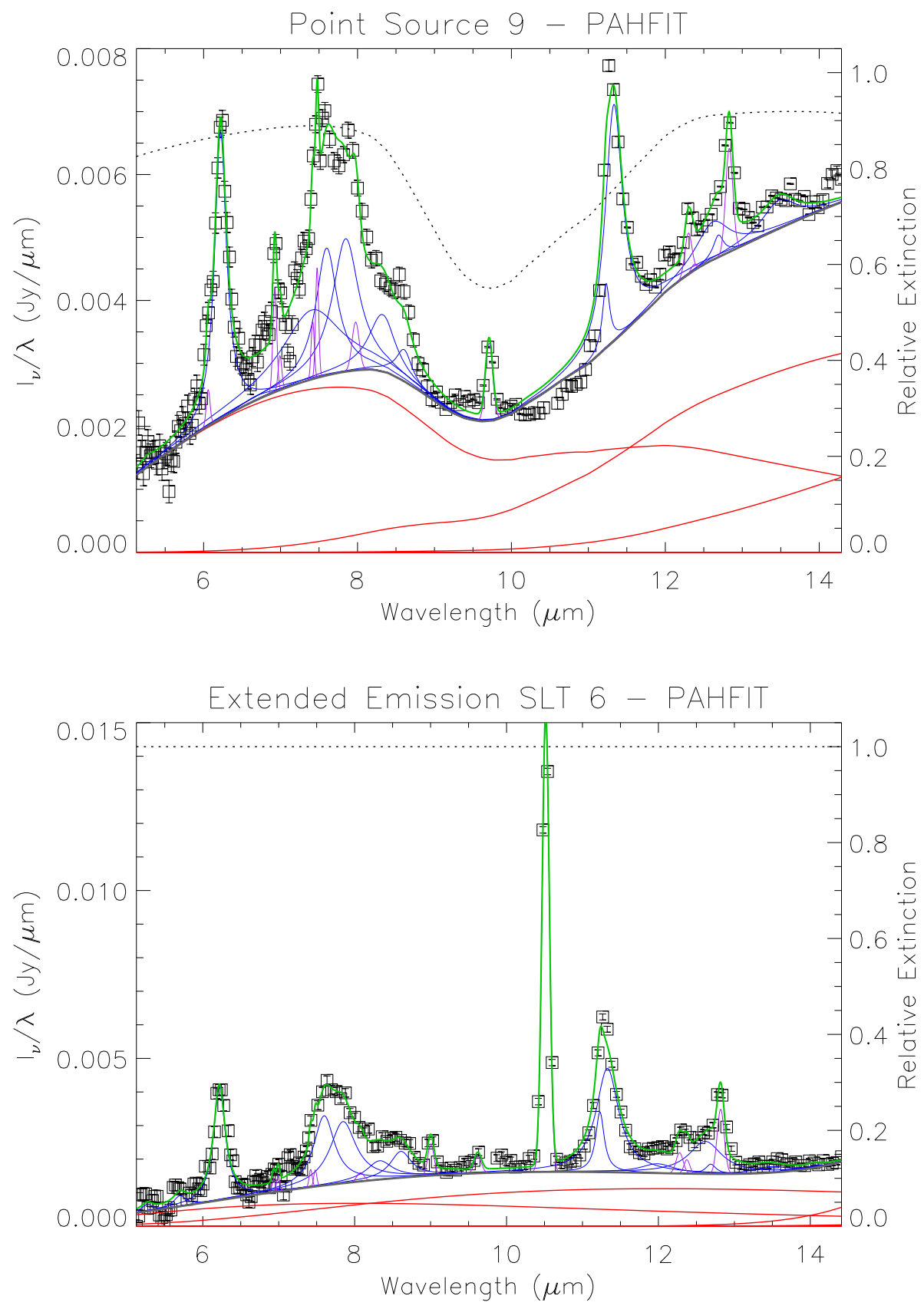

Figure 4.5: Example spectral fits with PAHFIT are shown for two sources, point source PS2 and the extended emission spectrum SLT6. The red lines are blackbody fits to the dust continuum, the blue features are PAHs, the narrow features in purple are atomic and molecular emission lines, the dotted line is the relative extinction and the green line is the composite fit to the data (squares). 
were recorded. This was done 300 times, after which the standard deviation of the

PAH feature strength measurements was taken as the statistical error in the 300 fitted feature strengths.

While PAHFIT also fits any number of user-requested atomic, ionic, and molecular features in addition to the dust continuum and PAH features, we fit the narrow lines manually with the IDEA tool in SMART. The reason for this additional step is that PAHFIT functions first and foremost as a fitting tool, and will therefore include all of the parameters it is given into the final solution. This means that in the point source spectra, for instance, PAHFIT will generally give the [SIV] $10.51 \mu \mathrm{m}$ line a flux even when it is clear from a visual inspectrion that this line is not detected. Therefore, in order to systematically determine narrow line fluxes and upper limits, we chose to use a manual tool for line fits. We used a first-order local continuum subtraction to estimate continuum flux beneath the narrow features. The [NeII $12.81 \mu \mathrm{m}$ feature is blended with a PAH feature and the line flux may therefore contain some PAH emission; due to the presence of PAHs in all spectra, we believe that any offset due to this contamination is largely systematic but may contribute substantially to the point source spectra PS12, PS13, and PS14, which exhibit much lower [NeII] fluxes than the other spectra. The continuum at 13.7-14.2 $\mu \mathrm{m}$ contains two prominent PAH features that, when compared to the PAHFIT dust continuum and PAH feature fits, systematically contributed roughly twenty per-cent to the continuum at these wavelengths; the continuum was therefore measured as the average in the PAHFIT dust continuum beneath the PAH 
features. The final atomic, ionic, PAH, and continuum measurements along with their errors are given in Tables 3-6.

\subsection{ANALYSIS}

\subsubsection{Point Source Identification}

We carried out a literature search in order to identify known objects associated with the infrared point source centroids. Table 1 lists the targeted point sources from this study, corresponding labels from Lebouteiller et al. [115] and Contursi et al. [38], and additional information from the literature. All point sources are identified as sites of recent or ongoing star formation, and are typically young protostars or young stellar clusters. A number of the point sources exhibit interesting spectral features that are worth a closer look. We describe these point sources in detail below.

\subsubsection{Young Star Clusters Exhibiting Silicates in Emission}

Unresolved emission from two bright star clusters in N66 exhibit silicate emission features in their spectra: NGC 346 (PS9) and N66B (PS6); see Figure 4.6. Both of these point sources are bright $\mathrm{H} \alpha$ sources [73], contain 'blue' stars [66, 128], and have been modeled as $\sim 3$ Myr old with Hubble color-magnitude diagrams 


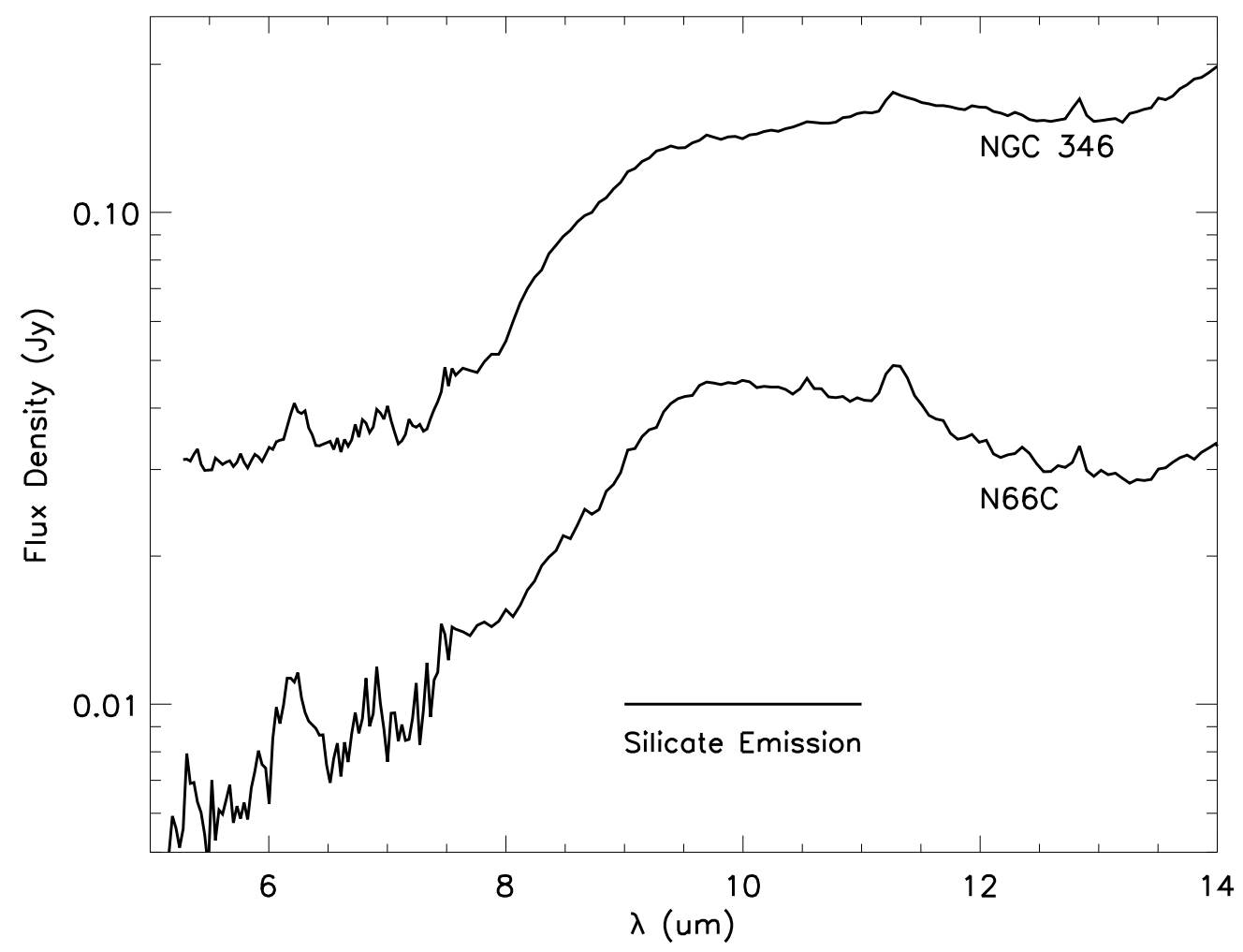

Figure 4.6: The spectra of two stellar clusters in the N66 giant HiI region: NGC 346 (PS9), the central source and brightest optically visible cluster, and N66B (PS6), a large stellar cluster to the north of NGC 346 (see Figure 4.2). Both show pronounced silicate emission features.

[169]. This age is consistent with the presence of remnant dust from the natal cloud surrounding the cluster.

The origin of the silicate emission is most likely an optically thin layer of intracluster and/or enveloping dust that has been heated to $\sim 200 \mathrm{~K}$ by the stars in the central clusters. The presence of silicate emission suggests a relatively low optical depth; this is supported by optical data of N66 [see, for example, the high-resolution Hubble data presented in 169], where the stars in the centers of the clusters are clearly visible through the intervening dust, and supported by 
the measured extinction by dust [23]. This geometry is similar to clumpy model geometries for young star clusters presented in Whelan et al. [218], where silicate emission/absorption was found to be dependent on the line-of-sight dust geometry. Neither cluster is resolved in the spectral slit, and we therefore did not employ the IRS LL module $(15<\lambda<37 \mu \mathrm{m})$ to measure the $17 \mu \mathrm{m}$ silicate emission as in Hao et al. [69], Sturm et al. [198] because the flux mis-match between the SL and LL Spitzer/IRS modules is very substantial and would bias the silicate dust temperature measurement. The presence of an $\mathrm{O} 5.5 \mathrm{~V}$ star in $\mathrm{N} 66 \mathrm{~B}$ and an $\mathrm{O} 9 \mathrm{~V}$ star in NGC 346 means that the point source extractions show a little [SIV] emission in N66B, none in NGC 346, and [NeII] emission in both. The [SIV] emission in N66B is likely due to the O5.5V star. See Sabbi et al. [170], Figure 7 for a map of the positions of the known O stars across N66, all of which lie off of the large stellar cluster positions.

Silicates in emission associated with young star clusters have not been observed regularly before. In NGC 3603, pointings on and near the central star cluster show silicate emission [114]. There is one pointing in 30 Doradus in the LMC [source B; 91, and also found in Lebouteiller et al. 2008] near R136 that exhibits silicate emission, but this source has been spectroscopically identified as an M-type supergiant star [148]. While there are numerous detections of silicates in emission among protoplanetary systems [e.g. 63, 188] and evolved stars such as asymptotic giant branch stars [AGBs, both galactically and extragalactically; see 118, 191], there are relatively few young stellar clusters that show silicate emission. Robberto 
et al. [161] show that a diffuse silicate population is likely across the Orion nebula.

Compared with those observations, the silicate emission observed in NGC 3603, NGC 346, and N66B is distinct in that it is clearly associated with the stellar clusters and not visibly dispersed across the region. It seems likely that the strong silicate emission associated with the star clusters in N66 and NGC 3603 is tied to a relatively short period of time in the early evolution of star clusters and will only last a short period of time; that these clusters are definitively young [e.g. 66] and therefore contain no AGB stars excludes the possibility of silicate-rich winds from post-main sequence stars contributing to the observed silicate emission.

\subsubsection{Silicate and PAH Emission Associated with a B [e] star}

There is a third spectrum exhibiting a silicate emission feature: PS8. In this instance, the silicate feature is not as pronounced as for the star clusters discussed above, though it has pronounced PAH features as well (see Figure 4.15 in the Appendix). Searching by position, we found a Be star at those coordinates, $\mathrm{Cl}^{*}$ NGC 346 KWBBE 200. Wisniewski et al. [223] fit a UV-to-8 $\mu$ m spectral energy distribution (SED) with a B-star template and a $\mathrm{T} \sim 800 \mathrm{~K}$ blackbody, observed P Cygni profiles on a number of optical spectral lines, roughly determined a luminosity of $10^{4.4} \mathrm{~L}_{\odot}$, and concluded that this source is a $\mathrm{B}[\mathrm{e}]$ supergiant. Evidence against it being a Herbig Be system is that no inverse $\mathrm{P}$ Cygni profiles associated with infall were observed, and that the derived luminosity is on the high end for Herbig Be stars. We note, however, that silicate emission and strong PAH bands 
are often detected in Herbig AeBe star systems [e.g. 103], where cooler dust and

PAHs are expected due to its young age. Additionally, PS8 exhibits a Class A PAH spectrum as shown in Figure 4.8 and discussed in $\S 4.5 .3$, which is typical for non-isolated Herbig AeBe stars [149], and is a $24 \mu \mathrm{m}$ source as observed by Spitzer/MIPS [160], suggesting that there is cold dust that Wisniewski et al.'s SED fit did not account for. While the absence of inverse P Cygni profiles offers a conundrum, the evidence from the mid-IR suggests that KWBBE 200 is, in fact, a Herbig AeBe star, not a B[e] supergiant.

\subsubsection{An Embedded Massive Young Stellar Object at the Edge of HiI Region N66A}

The single brightest mid-IR point source in N66, PS7, lying at the location of the HII region N66A [73], was found to be a $16.6 \mathrm{M}_{\odot}$ class I young stellar object (YSO) by Simon et al. [189] using Spitzer/IRAC and MIPS photometry matched to models of class I protostars presented in Robitaille et al. [163]. Shown in Figure 4.7, the spectrum has a deep silicate feature at $9.8 \mu \mathrm{m}$ which corresponds to high optical depth and therefore supports the class I designation. Furthermore, the presence of $\mathrm{H}_{2} \mathrm{O}$ ice in the $6-7.5 \mu \mathrm{m}$ range and a $\mathrm{CO}_{2}$ ice feature at $15 \mu \mathrm{m}$ in the high-resolution Spitzer spectrum of N66A suggests cold, dense conditions similar to other massive class I YSO environments [e.g. 122]. Lastly, there is no [SIV] or [NeII] detected, suggesting that the central heating source does not have 


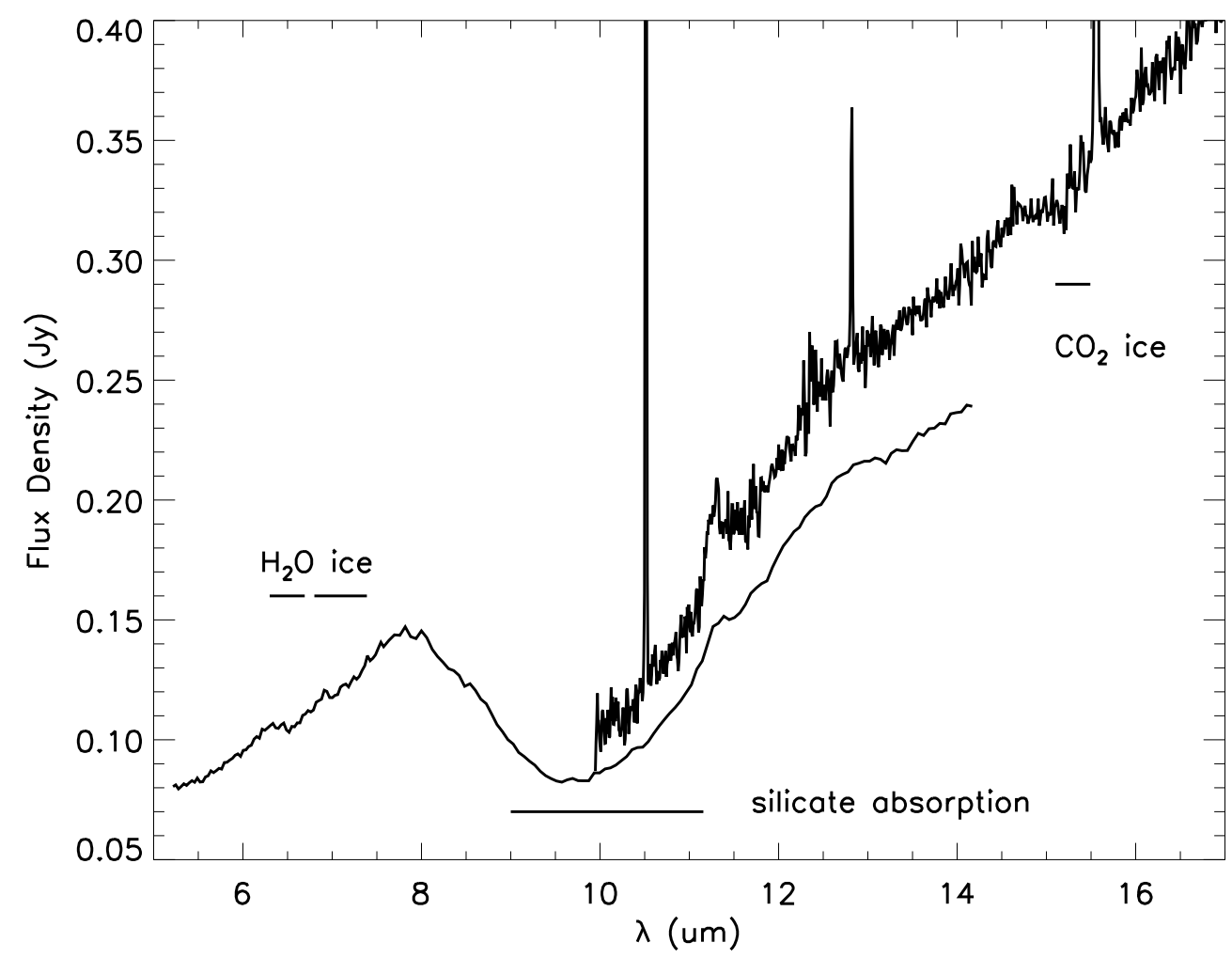

FIGURE 4.7: The Spitzer/IRS SL/SH spectrum of N66A exhibits features commonly associated with a massive young stellar object: water ice features between 6-7.5 $\mu \mathrm{m}$, deep silicate absorption indicative of high optical depth, $\mathrm{CO}_{2}$ ice at $15 \mu \mathrm{m}$, and a strong mid-infrared continuum. The nebular lines seen in the high-resolution spectrum come from intervening diffuse material.

a strong UV continuum. These spectroscopic signatures all confirm Simon et al.'s original designation.

To confirm the YSO mass, we fit Spitzer/IRS data (wavelength coverage from 5 to $35 \mu \mathrm{m})$ with the Robitaille et al. models ${ }^{2}$. For these fits of an embedded protostar, stellar temperature (and mass), disk/envelope mass and inner/outer radii are all fit, though for embedded sources disk masses are known to be ill-constrained. The best fit to our data was a $17.8 \mathrm{M}_{\odot}$ YSO embedded in an envelope of about $10^{3} \mathrm{M}_{\odot}$.

\footnotetext{
${ }^{2}$ We used the SED fitting routine described in Robitaille et al. [164] and available to the public via: http://caravan.astro.wisc.edu/protostars/
} 
The circumstellar extinction is calculated to be $\mathrm{A}_{V}=25.7$, and the interstellar extinction is $\mathrm{A}_{V}=0.1$. For comparison, $\tau_{9.8 \mu m}=1.88$ from the PAHFIT parameter fit to this source, making the $9.8 \mu \mathrm{m}$ feature, as for many heavily embedded objects [31], an unreliable determinant of column density of the embedding envelope. The total luminosity of this model is $3.47 \times 10^{4} \mathrm{~L}_{\odot}$. This result differs from the fit presented in Simon et al. [189] by a $\sim 7 \%$ increase in mass and $\sim 15 \%$ increase in luminosity.

Heydari-Malayeri \& Selier [75] studied the Hubble data for the Hit region N66A in great detail and determined that it is supported by an $\mathrm{O} 8$ star. While the massive YSO discussed in this section dominates the infrared emission, the fine structure emission in the extended emission spectrum is due to the young O- and B-star population in N66A, while the diffuse dust emission probably comes from the PDR at the interface to the molecular cloud in which the massive YSO is buried. There are in fact two YSOs at PS9's centroid in the Simon et al. [189] atlas; the more massive YSO studied here dominates the infrared luminosity substantially: there is a factor of about 19 ratio between the luminosities of the two YSOs. At the sensitivity of these data, the massive YSO is the only point source detected. 


\subsubsection{Ionic Lines}

Ionic emission lines in an HII region can help quantify the strength and hardness of the radiation [see 117] but may also be used to shed light on the physical characteristics of the point sources and diffuse emission across the region. In general, the point source spectra are all associated with sites of active star formation. By contrast, the extended emission spectra trace the HII and PDR emission that is photoexcited by the massive stellar population dispersed across the region. For this dataset, we find two distinguishing features in the ionic line emission that are specially worth noting.

(1) [SIV] $10.51 \mu \mathrm{m}$ emission is largely undetected among the point source spectra but is detected in all of the extended emission spectra.

(2) [NeII $12.81 \mu \mathrm{m}$ is detected in every point source and extended emission spectrum with only two exceptions among the point source spectra.

The strong [SIV] line, due to ionization of the interstellar gas by the O star population, is seen in the extended emission spectra across the region (see Figures 4.17 and 4.18). [SIV] and [NeII] are often found in sites of active star formation, e.g. giant HiI regions [115], blue compact dwarf galaxies [BCDs; 229], and starburst galaxies $[12,18]$. Our analysis differs from previous works by separating the diffuse emission from the infrared point source emission. We show that the dense regions embedded in the PDR do not generally show [SIV] emission. The absence of [SIV] in most of the point source sample is likely because the heating sources at the 
point source locations are not hard enough to triply-ionize sulfur. The gas density at these positions can be estimated from the [SIII] $18.71 \mu \mathrm{m}$ and $33.48 \mu \mathrm{m}$ lines fluxes from high-resolution Spitzer data at the point source positions published in Lebouteiller et al. [115]. The ratio of these two lines ranges from 0.43-2.7, suggesting densities of $\mathrm{n}_{e} \mathrm{~T}_{4}^{1 / 2}<3 \times 10^{3} \mathrm{~cm}^{-3}$ for the lowest value ratio and below about $10^{2} \mathrm{~cm}^{-3}$ for most of the positions, well less than the critical densities of $n_{\text {crit }}([\mathrm{SIV}])=5.4 \times 10^{4} \mathrm{~cm}^{-3} n_{\text {crit }}([\mathrm{NeII}])=6.5 \times 10^{5} \mathrm{~cm}^{-3}$. It should be noted that these estimates for the [SIII]-derived gas density are subject to the diffuse as well as the dense material at these positions because the point source emission cannot be treated separately from the diffuse emission in the high-resolution modules as it can for the low-resolution modules.

\subsubsection{PAH Feature Profiles}

In order to classify the PAH spectra observed in N66, we plot only the highest signal-to-noise $(\mathrm{S} / \mathrm{N})$ N66 PAH spectra versus templates in the 7-9 $\mu \mathrm{m}$ range and 11-12 $\mu \mathrm{m}$ range from Peeters et al. [149] and van Diedenhoven et al. [44] respectively in Figures 4.8 and 4.10 . Peeters et al. was able to classify the 6$9 \mu \mathrm{m}$ PAH features based on their peak centroids, and discovered that there is a relationship with environment: Class A spectra are typical for Hit regions and nonisolated Herbig AeBe stars, Class B spectra are more typical for isolated Herbig AeBe stars, and Class C spectra are more common in evolved stellar systems. van 

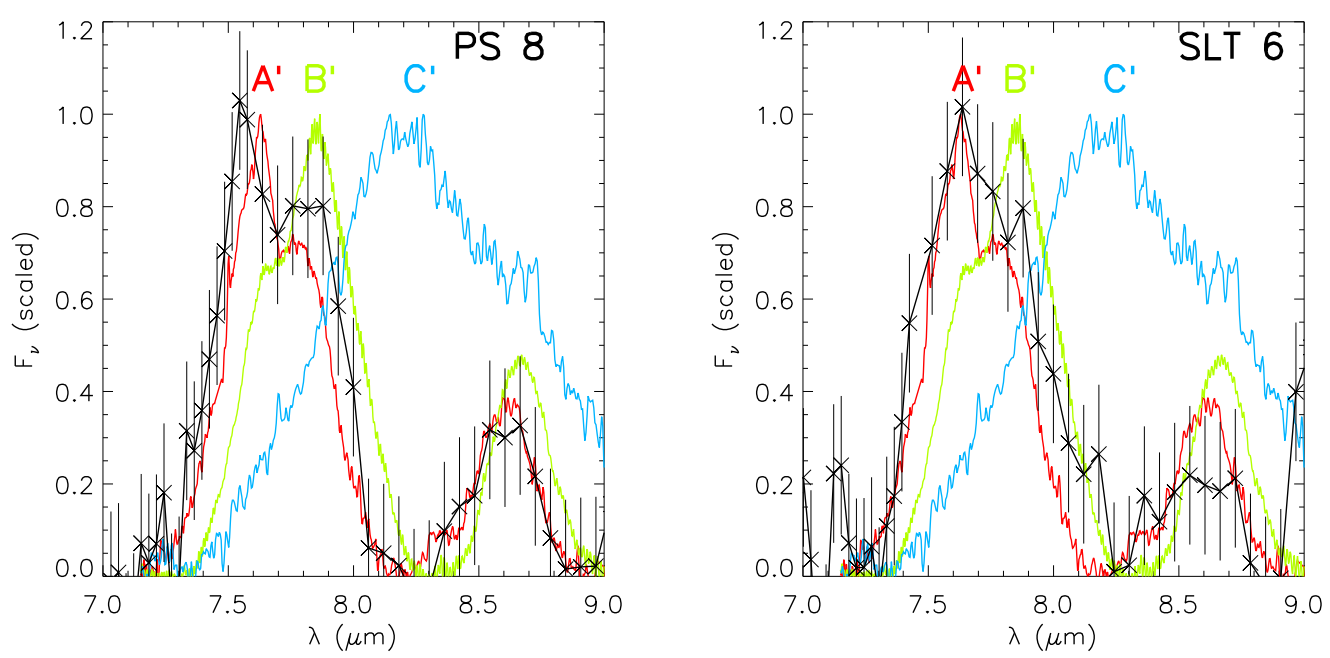

Figure 4.8: Example $7.7 \mu \mathrm{m}$ and $8.6 \mu \mathrm{m}$ PAH features are plotted versus the Peeters et al. [149] templates (templates are from ISO SWS, with $\mathrm{R} \sim 450$ for classes $\mathrm{A}$ and $\mathrm{C}$ and $\sim 1500$ for class $\mathrm{B}$ ), showing both the width of the $7.7 \mu \mathrm{m}$ feature and the suppressed $8.6 \mu \mathrm{m}$ emission in the right panel. For the spectra of the extended emission, the strong lines at $7.46 \mu \mathrm{m}$ and $8.99 \mu \mathrm{m}$ are Hi 6-5 and [ArIII] respectively. The N66 spectra by and large resemble the class A template, as expected for a giant HiI region. Spectra were scaled so that the peak of the $7.7 \mu \mathrm{m}$ feature matched that of the templates.

Diedenhoven et al. studied the $11.3 \mu \mathrm{m}$ PAH complex and developed templates for Classes A and B only.

The N66 spectra for the $7.7 \mu \mathrm{m}$ and $8.6 \mu \mathrm{m}$ features (Figure 4.8) are generally most similar to the Class A template, as is expected for an Hir region. However, in some of the spectra, the $7.7 \mu \mathrm{m}$ features appear to be wider than the templates. For the $11.3 \mu \mathrm{m}$ PAHs, the observed line centers appear to be slightly redshifted with respect to the Orion Nebula (Figure 4.9); i.e. less than one resolution element. However, this is the only feature in the N66 spectra, ionic, molecular, or PAH, which shows a centroid shift, and while such a systematic offset could very well be due to continuum subtraction it is deserving of closer attention. 

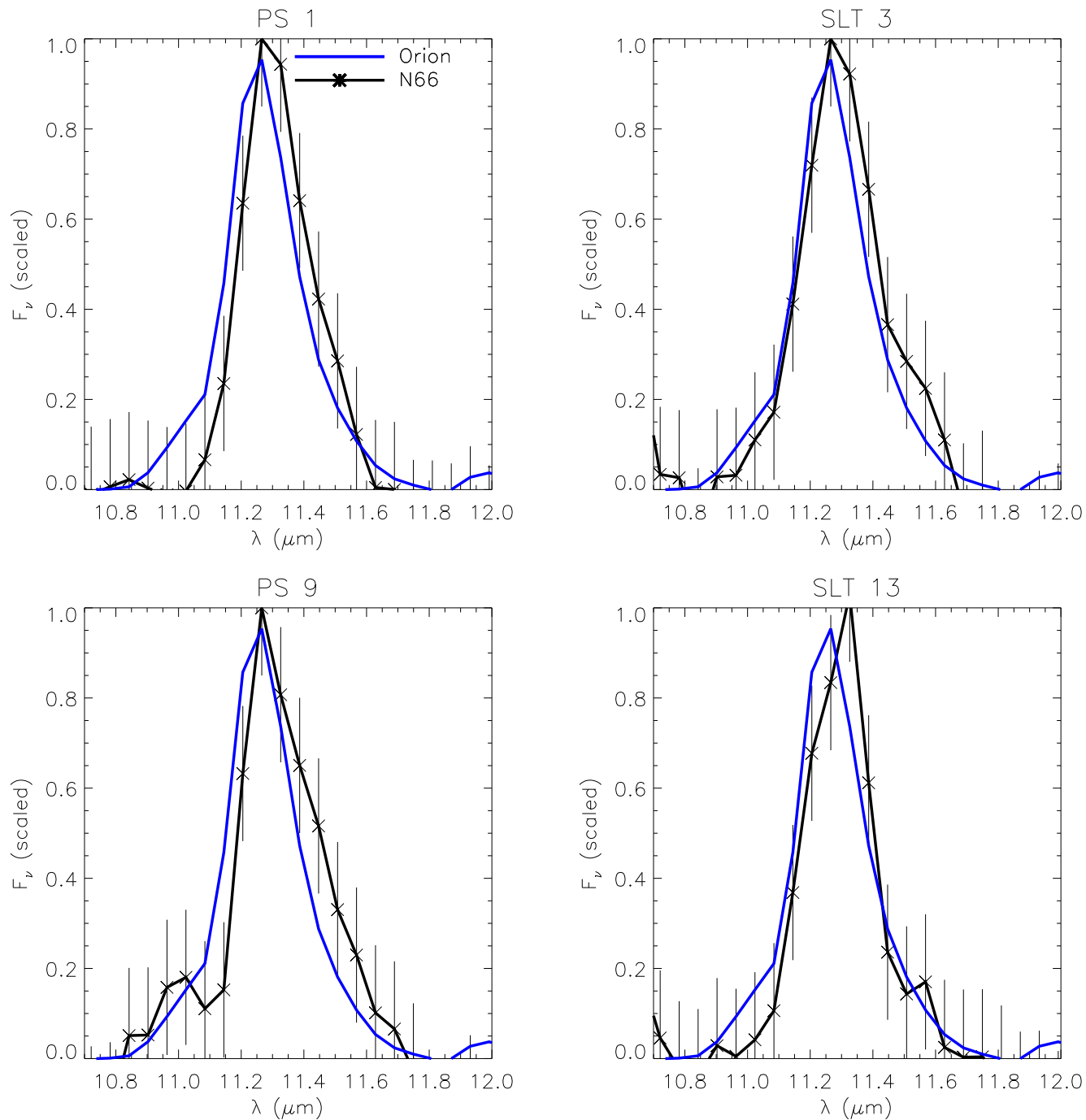

FiguRE 4.9: Example $11.3 \mu \mathrm{m}$ PAH spectra in black are plotted against a SL spectrum of the Orion nebula. The N66 spectra appear to have peaks that are redward of the Orion spectra.

Therefore, the available high-resolution spectra $(\mathrm{R} \sim 600)$ of N66 were reduced for comparison with the PAH templates from van Diedenhoven et al. [44] (Figure 4.10). Labelling for these spectra, first used in Lebouteiller et al. [115], follows that work. Unfortunately, the SH data have a low S/N. However, in the spectra for positions 1, 2, 3, and 5, a narrow line, 2-3 wavelength elements wide, that corresponds to the position of the Hi 9-7 $11.31 \mu \mathrm{m}$ line, is visible. Due to the fact that the raw 
images do not reveal single pixels with high values at this wavelength, it is not likely that this line is due to improperly calibrated pixels on the array, but is an actual astronomical feature. In order to determine whether the narrow line at $11.3 \mu \mathrm{m}$ is the Hi 9-7 line, we compared the line strength to that of the detected Hi 7-6 $12.37 \mu \mathrm{m}$ line to see if their ratio is consistent with case B recombination theory [85]. We used a temperature of $12,500 \mathrm{~K}$ and a number density of $10^{2} \mathrm{~cm}^{-3}$ to compare to the data; N66 has measured values of 12,269 K average and 50$500 \mathrm{~cm}^{-3}[52,145,151,209]$. For Case B,

$$
\frac{H I 9-7}{H I 7-6}=0.223
$$

For sources 1,2 , and 3 , the ratio was about 0.2 , and for source 5 , it was $<0.1$. Considering the low $\mathrm{S} / \mathrm{N}$ of these data, but also considering the other detected Hi lines (Hi 7-6 line in hi-res and the Hi 6-5 line at lo-res), it is likely that the detected line is the Hi 9-7 line and that this line is contributing to the apparent redshift of the $11.3 \mu \mathrm{m}$ feature.

Leaving aside the narrow line, there are still several cases in which the peak of $11.3 \mu \mathrm{m}$ PAHs in N66 are slightly redward $(\Delta \lambda \sim 0.1 \mu \mathrm{m})$ of the two templates (positions 8, 9, 10 and 13 show it most clearly in spite of the low $\mathrm{S} / \mathrm{N}$ ). According to the Spitzer IRS instrument handbook ${ }^{3}$ the wavelength calibration is good to $1 / 5$ of a resolution element, or, for $\mathrm{SH}, \sim 0.001 \mu \mathrm{m}$. Pointing offsets are also a minor

${ }^{3}$ The IRS instrument handbook may be found online at:
http://irsa.ipac.caltech.edu/data/SPITZER/docs/irs/irsinstrumenthandbook/


concern, but could produce a 0.5-pixel shift, or $\sim 0.04 \mu \mathrm{m}$. Both of these potential errors are small in comparison to the $\sim 0.1 \mu \mathrm{m}$ shift in the PAH feature centroid, would additionally affect all features in this spectral order, and therefore cannot account for the shift seen. We must stress once again that continuum subtraction may play a role in the centroid mismatch. If born out, this is the only ISM-type environment observed so far that exhibits a profile redshifted compared to class A. We also note the absence of the $11.0 \mu \mathrm{m}$ feature seen in the templates, though its absence is likely due to the low $\mathrm{S} / \mathrm{N}$ of these spectra.

While the observed wavelength shifts across N66 appear in both point source and ISM spectra, a worthwhile comparison is Herbig AeBe stars, which often exhibit $11.3 \mu \mathrm{m}$ PAH peaks that are shifted redward by a similar amount. Keller et al. [103], Sloan et al. [192] show peak shifts in both the $7.7 \mu \mathrm{m}$ and $11.3 \mu \mathrm{m} \mathrm{PAH}$ complexes. It is interesting that we do not see an appreciable shift in the $7.7 \mu \mathrm{m}$ feature, which is often shifted by as much as $0.3 \mu \mathrm{m}$ or more in Herbig AeBe environments and would therefore be detectable with our spectra.

Because the redward shift of the $11.3 \mu \mathrm{m}$ PAH complex is small $(0.1 \mu \mathrm{m})$ and the detected Hi 9-7 line must be resolved out of the feature, we recommend that high-resolution and high $\mathrm{S} / \mathrm{N}$ follow-up observations be made. JWST's MIRI instrument, with its spectral resolution of $\sim 3000$, is a perfect candidate instrument for such follow-up. Such observations would be capable of not only confirming the wavelength shift of the PAH and more accurately determining the line flux and width of the narrow line, but, an expanded high-resolution study could be used to 

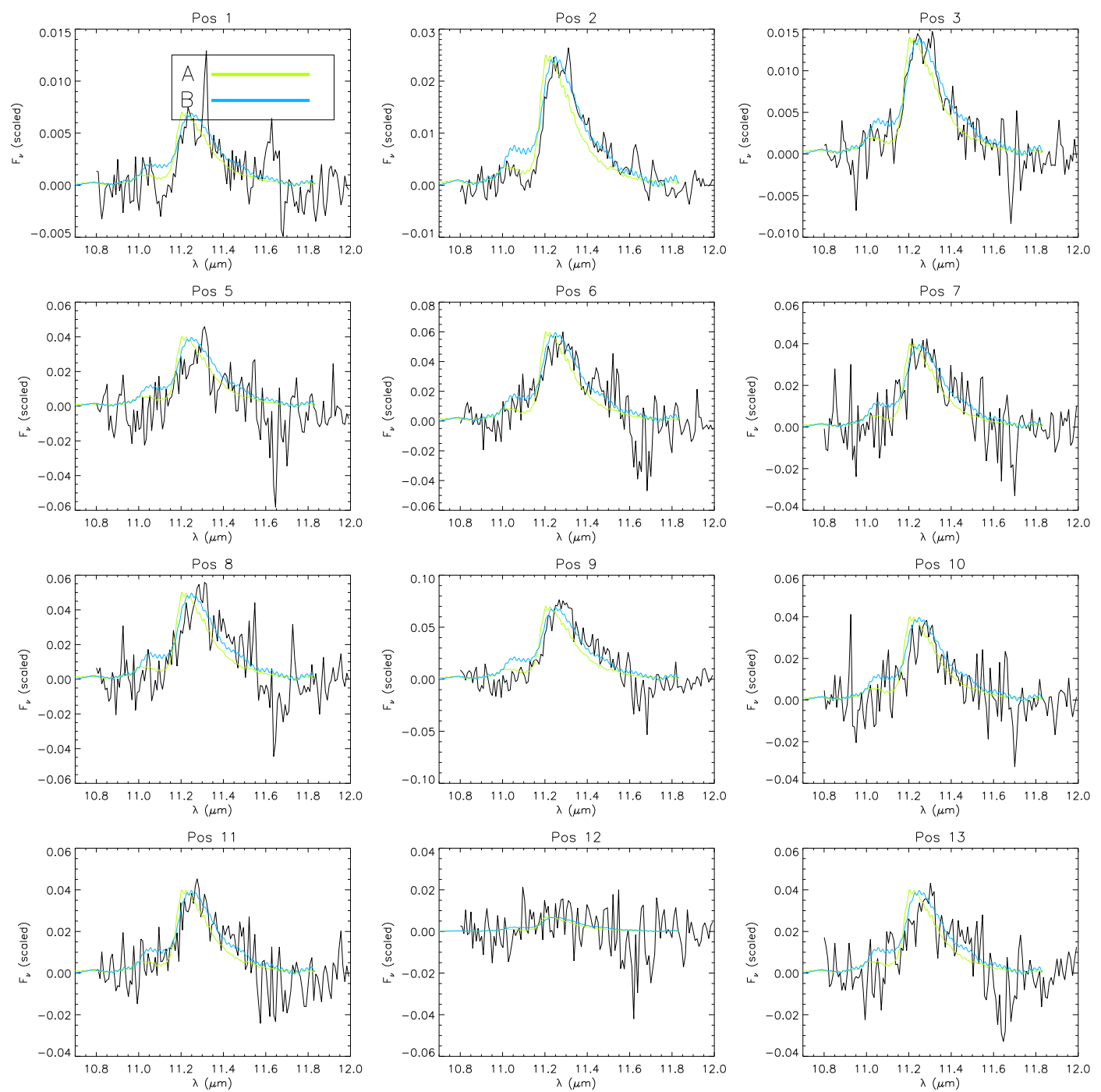

Figure 4.10: The $11.3 \mu \mathrm{m}$ PAH for all of the IRS SH pointings are plotted versus class A and class B PAH template spectra from van Diedenhoven et al. [44]. The N66 spectra are scaled to best fit the templates, excluding the possible Hi 9-7. For several sources, there are prominent shifts that are independent of the HI line (most notably position $8,9,10$, and 13 ). 
Chapter 4. An In-Depth View of the Mid-Infrared Properties of Point Sources and the Diffuse ISM in the SMC Giant HII Region, N66

determine whether these features are peculiar to N66 (or, perhaps, the SMC), or are systematic at low metallicity. 


\subsection{4 $14 \mu \mathrm{m}$ Continuum Emission}

The continuum between 13.5 and $14.2 \mu \mathrm{m}$ is due to emission by a combination of very small carbonaceous dust grains [VSGs: a > $50 \AA$; 121] and warm large dust grains. However, we expect the VSGs to dominate the emission at these wavelengths; Draine \& Li [51], $\S 5$, notes that the VSG contribution to the continuum at $\lambda<20 \mu \mathrm{m}$ is roughly independent of the radiation field strength because it results from single-photon heating.

The $14 \mu \mathrm{m}$ continuum is measured as the continuum beneath the PAH bands as fit with PAHFIT. Figure 4.11 plots the $14 \mu \mathrm{m}$ continuum over [SIV] versus the radiation field hardness as traced by $[\mathrm{SIV}] /[\mathrm{NeII}]$ for the extended emission spectra. By normalizing the $14 \mu \mathrm{m}$ continuum emission by the [SIV] line flux, we are able to compare the dust continuum emission to the radiation field strength, and then plot that value versus the radiation field hardness. There is an anti-correlation that suggests that the $14 \mu \mathrm{m}$ continuum is weakest where the radiation field is hardest and strongest. Weaker continua in harder and stronger radiation fields supports the assertion first presented in Contursi et al. [38] that the VSGs are likely being photodestroyed by the $\mathrm{U}=10^{5} \mathrm{ISRF}$ radiation field in N66.

Because the correlation seen in Figure 4.11 is roughly linear excluding the outlier, this supports the assertion that the $14 \mu \mathrm{m}$ emission is mostly due to VSGs, not large grains; if there was a substantial contribution from large grains to the $14 \mu \mathrm{m}$ 


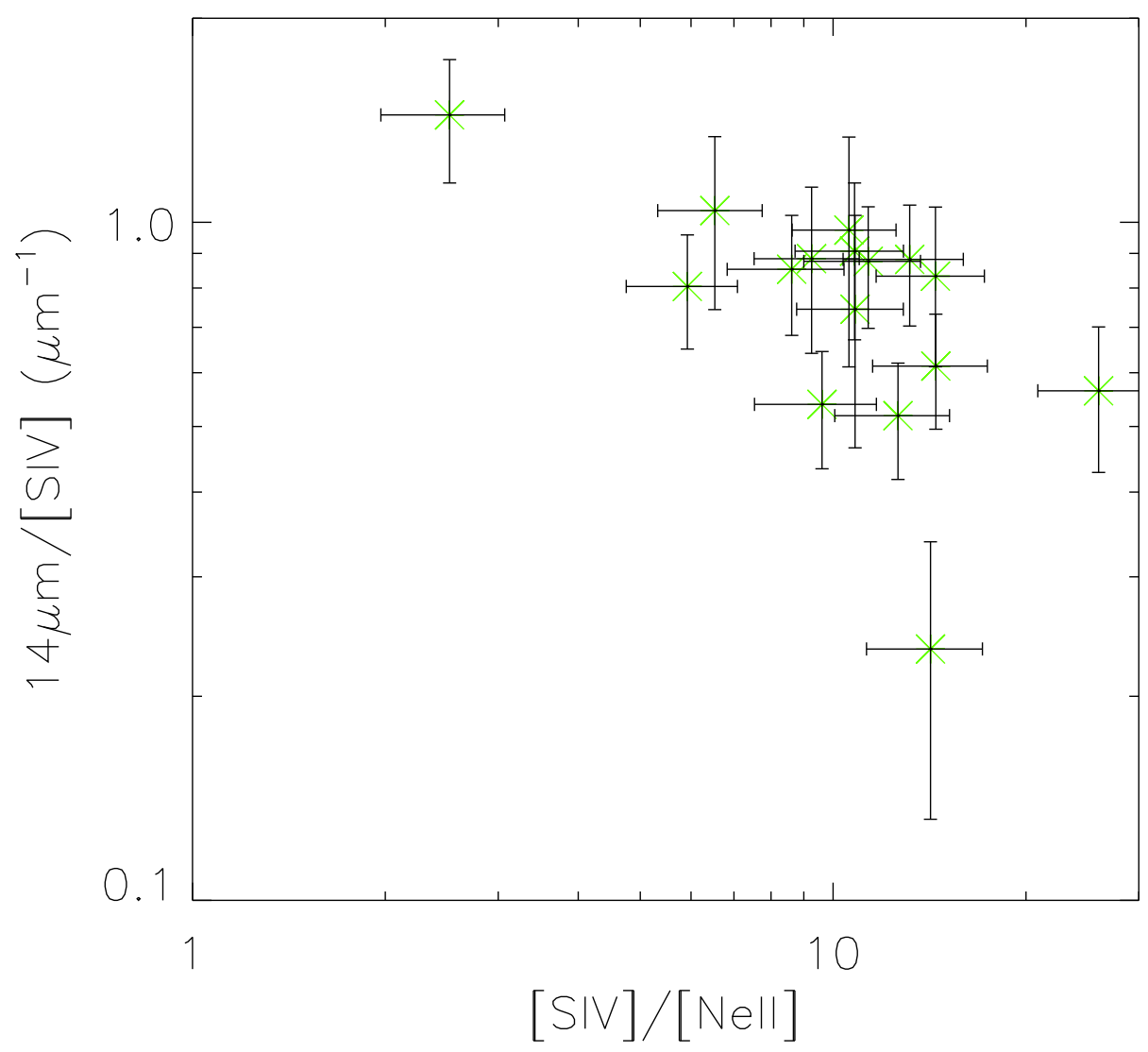

Figure 4.11: The $14 \mu \mathrm{m}$ continuum divided by [SIV] flux in the extended emission versus $[\mathrm{SIV}] /[\mathrm{NeII}]$. These data show an anti-correlation that suggests that the $14 \mu \mathrm{m}$ continuum is weakest in the hardest and strongest radiation fields.

continuum, then the expected variations in large grain dust temperature across the region would make this trend non-linear.

\subsubsection{PAH Ratios as Diagnostics}

There are four major PAH features in the 5-15 $\mu \mathrm{m}$ wavelength range. These features are identified by specific resonant modes inside the PAHs $[2,51]$. (1) The 
$6.2 \mu \mathrm{m}$ feature is created by an aromatic C-C stretch mode. (2) The $7.7 \mu \mathrm{m}$ feature is also due to C-C aromatic stretch modes. (3) The $8.6 \mu \mathrm{m}$ feature is emitted by a $\mathrm{C}-\mathrm{H}$ in-plane bending mode, while (4) the $11.3 \mu \mathrm{m}$ feature is due to a solo $\mathrm{C}-\mathrm{H}$ outof-plane bending mode and is sensitive to edge structure [2, 80, 157]. Furthermore, the 6.2, 7.7, and $8.6 \mu \mathrm{m}$ PAH features are attributed to ionized PAHs, whereas the $11.3 \mu \mathrm{m}$ PAH feature is attributed to neutral PAHs [84], and there is believed to be a wavelength-size dependence for PAHs, wherein larger PAHs emit most efficiently in the longer-wavelength PAH features $[51,178]$.

PAH ratios offer an independent assessment of the ISM's physical characteristics. Galliano et al. [64] found an average correlation between the $\mathrm{I}_{7.7} / \mathrm{I}_{11.3}$ and $\mathrm{I}_{6.2} / \mathrm{I}_{11.3}$ for a sample of starbursts, BCDs, HII regions and PDRs, reproduced in Figure 4.12, where Galliano's average is shown as a solid line with the one- $\sigma$ standard deviation plotted on either side of the average. The data points for N66 are also plotted, with the point source data as red triangles and the extended emission data as green stars. The point source data show a range of $\mathrm{I}_{7.7} / \mathrm{I}_{11.3}$ from $\sim 0.03-6.3$, over two orders of magnitude, in a relatively narrow range of $\mathrm{I}_{6.2} / \mathrm{I}_{11.3} \sim 0.5-1.8$. The extended emission spectra have respective ranges of 0.35-8.4 and $\sim 1-3$. Averaging together all values for the $\mathrm{I}_{6.2} / \mathrm{I}_{11.3}$ and $\mathrm{I}_{7.7} / \mathrm{I}_{11.3}$ ratios weighted by feature strength, the point sources have values of 1.1 and 2.7 and the extended emission have values of 1.4 and 3.7 respectively. These averages are relatively close together and lie within the galaxy sample's average correlation.

However, the wide spread around those averages suggests an intriguing variety of 
physical attributes. For instance, the massive embedded YSO (PS7, discussed in detail in Section 4.5.1.3) has a very high $\mathrm{I}_{7.7} / \mathrm{I}_{11.3}$ ratio value; see Figure 4.12. This might suggest that the PAH emission from the embedding envelope is either more heavily ionized than other point sources, or else the average PAH size is smaller. Having a population of ionized PAHs in the YSO environment seems unlikely because the temperature of a Class I YSO is probably very low $[250 \mathrm{~K} ; 140]$. The Hir regions N66B and N66C (PS5 \& PS6) both lie near the average value for $\mathrm{I}_{7.7} / \mathrm{I}_{11.3}$, but NGC 346 (PS9) lies well off of this average, with an outlying value of $\sim 0.03$. The low value for the $7.7 \mu \mathrm{m}$ feature strength in NGC 346 is confirmed with a visual inspection of the spectrum - see the Appendix, Figure 4.16. As an opposite example to the YSO, NGC 346 may either contain a largely neutral PAH population or else very large PAHs; the latter solution seems most sensible given the numerous blue stars in the cluster.

The lack of correlation seen in Figure 4.12 is intriguing because the $6.2 \mu \mathrm{m}$ and $7.7 \mu \mathrm{m}$ PAH features are generally very tightly correlated for spectra of HiI regions and entire galaxies [64, 212], for planetary nebulae [13], and in the reflection nebula NGC 2023 (Peeters et al. 2013 in preparation). We have already determined that this is not due to the decomposition method (see $\S 4.4 .3$ ). The same lack of trend can be seen with the column-extracted PAH fluxes from Lebouteiller et al. [117], although a weak correlation may be seen in the Sandstrom et al. [174] results for the high $\mathrm{S} / \mathrm{N}$ data points. We are not aware of any other mention in the literature of a decoupling of the $6.2 \mu \mathrm{m}$ and $7.7 \mu \mathrm{m}$ PAH features in this manner. 


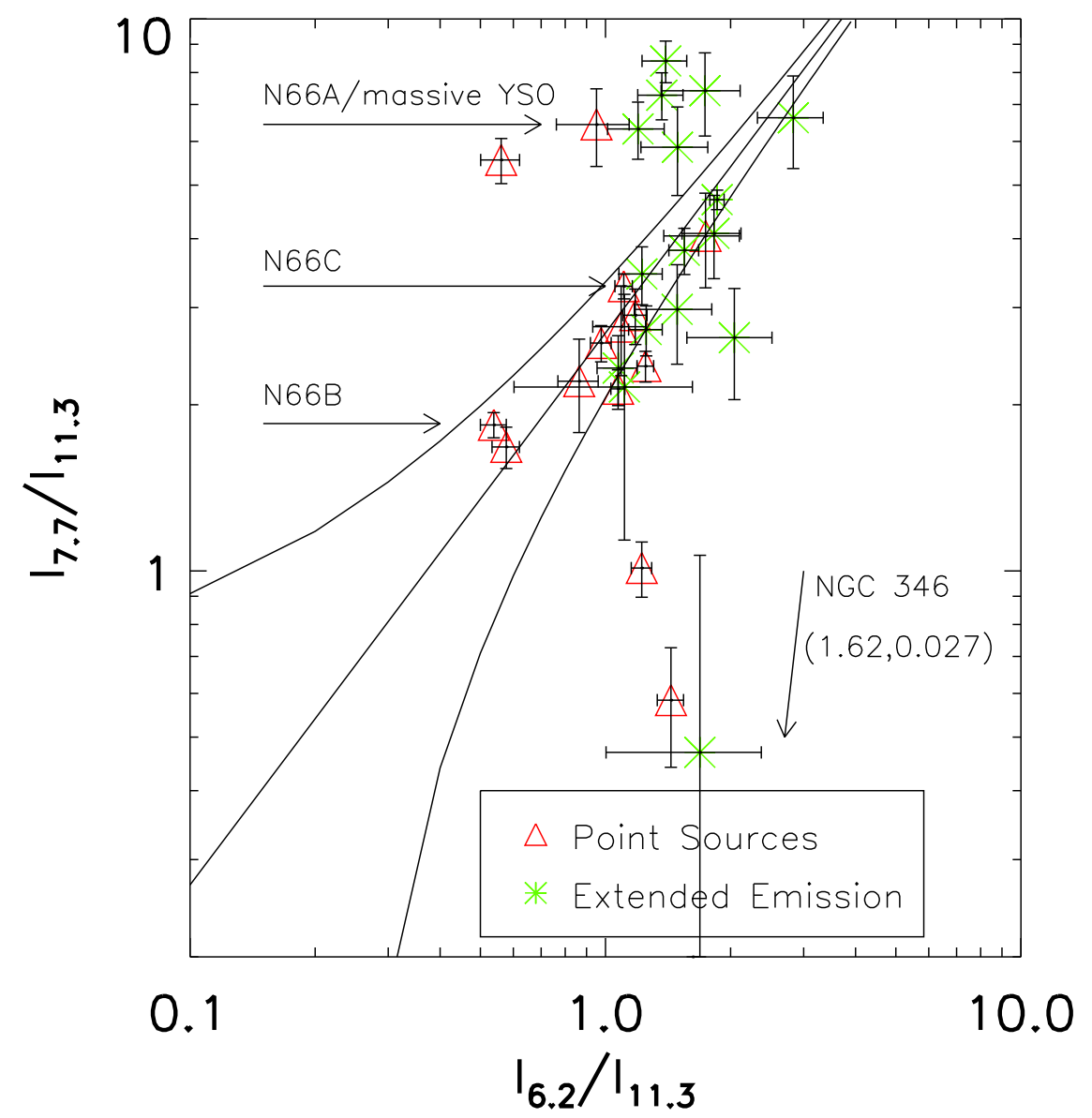

Figure 4.12: The average $\mathrm{I}_{7.7} / \mathrm{I}_{11.3}$ versus $\mathrm{I}_{6.2} / \mathrm{I}_{11.3}$ from Galliano et al. [64] Figure 3 is plotted as the solid line with its $1-\sigma$ spread shown. The N66 data points are overplotted.

To study general trends, the $\mathrm{I}_{7.7} / \mathrm{I}_{6.2}$ and $\mathrm{I}_{8.6} / \mathrm{I}_{6.2}$ are plotted against $\mathrm{I}_{7.7} / \mathrm{I}_{11.3}$ in Figure 4.13. A trend is seen in the $\mathrm{I}_{7.7} / \mathrm{I}_{6.2}$ versus $\mathrm{I}_{7.7} / \mathrm{I}_{11.3}$ ratios plot that was not recorded in the Galliano et al. dataset. The values of the $\mathrm{I}_{7.7} / \mathrm{I}_{11.3}$ ratio seem to suggest that the areas of extended emission (on the right) are largely photoionized whereas the point sources (on the left) are more neutral, with some exceptions. The variation of the $\mathrm{I}_{7.7} / \mathrm{I}_{6.2}$ ratio might suggest some photoprocessing effect: the $6.2 \mu \mathrm{m}$ PAH flux that is becomes weaker in harder radiation fields due to the 
destruction of the smaller PAH molecules.

There is no trend between the $\mathrm{I}_{8.6} / \mathrm{I}_{6.2}$ and $\mathrm{I}_{7.7} / \mathrm{I}_{11.3}$ PAH ratios. Because the $\mathrm{I}_{8.6} / \mathrm{I}_{6.2}$ ratio should be independent of ionization state and therefore should trace the size of the PAHs, while the $\mathrm{I}_{7.7} / \mathrm{I}_{11.3}$ ratio does trace ionization state, it appears that the size distribution is independent of ionization state. This includes both point sources and extended emission spectra, and it should be noted that a spread of about one order of magnitude still exists in the $\mathrm{I}_{8.6} / \mathrm{I}_{6.2}$ ratio values. We therefore conclude that N66 shows no evidence of local variation in the $\mathrm{N}_{C}^{\min }$ value due to the ionization state for the PAH population, but it is unclear how this result should compare to galaxies with significantly different metallicity or star formation activity.

In Sandstrom et al. [174], the weak $7.7 \mu \mathrm{m}$ feature across the entire SMC, including N66, and the relatively strong $11.3 \mu \mathrm{m}$ feature suggested to the authors that PAHs across the SMC are both small and mostly neutral. However, the authors used a stringent signal-to-noise cut-off, thereby excluding extended emission from their analysis and biasing their results towards the conditions found for our point source spectra. When our data are compared against the grey data points in the PAH ratios plots in Sandstrom et al. [174], it is interesting to note that the results from the map agree well with our targeted spectroscopy results. What our analysis is able to show is that the point sources lie in a similar PAH ratio space to the extended emission, despite the different physical conditions as discussed. Furthermore, it is clear that the PAH population exists on a spectrum, from more 


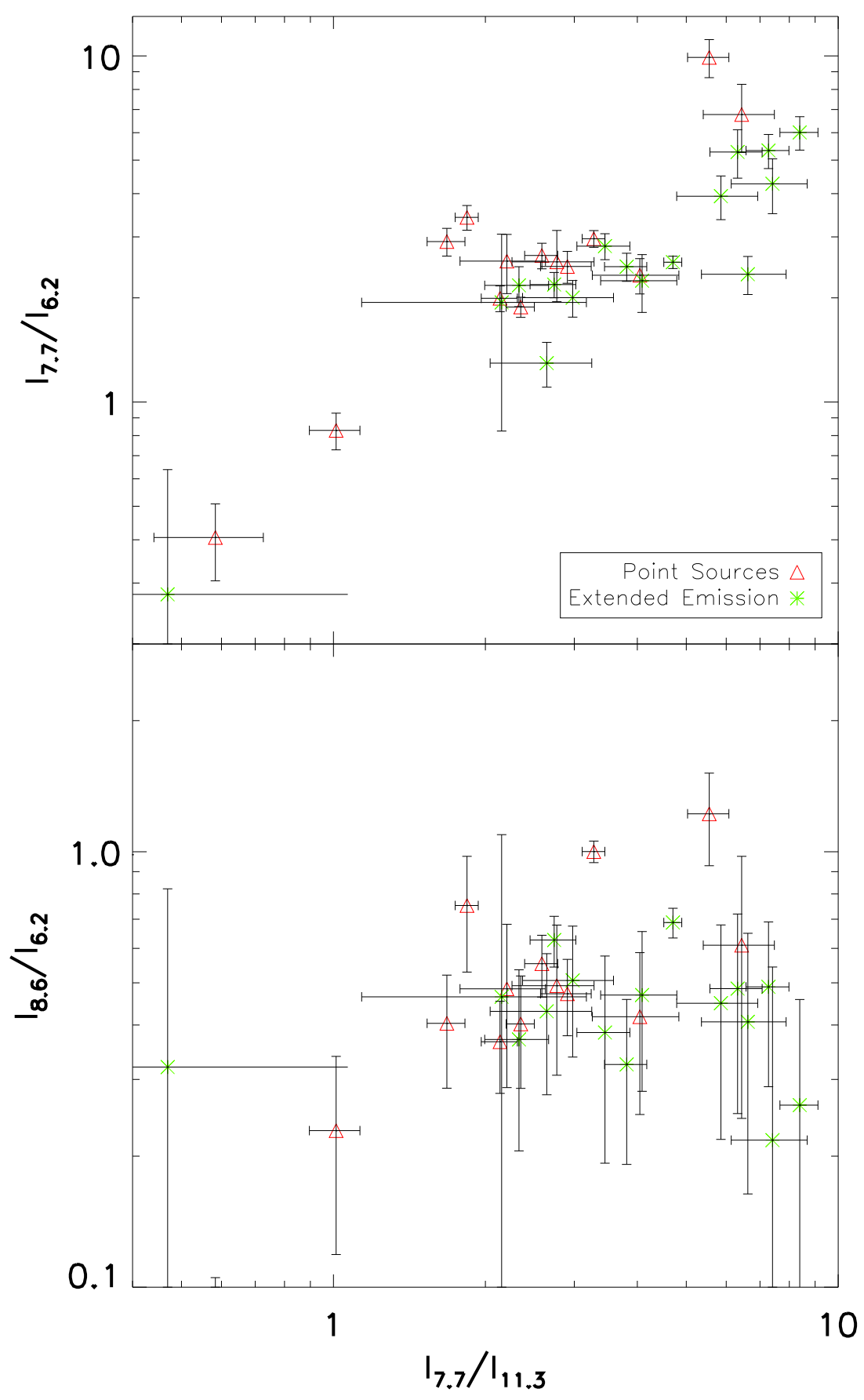

Figure 4.13: The $\mathrm{I}_{7.7} / \mathrm{I}_{6.2}$ and $\mathrm{I}_{8.6} / \mathrm{I}_{6.2}$ versus $\mathrm{I}_{7.7} / \mathrm{I}_{11.3}$ PAH ratios are plotted for N66. 
neutral in the dense PDR where the point sources lie, to more ionized in the diffuse PDR and HiI region.

The PAH population size distribution in low-metallicity environments is a contentious topic. In a study of low-metallicity BCDs, Hunt et al. [88] found relatively strong $\mathrm{I}_{8.6}$ and $\mathrm{I}_{11.3}$ features and interpreted this finding to mean a large $\mathrm{N}_{C}^{\min }$ value for PAHs. The analysis of the $\mathrm{I}_{17} / \mathrm{I}_{11.3}$ PAH ratio by Smith et al. [193] suggests the opposite, that low-metallicity environments $(12+\log (\mathrm{O} / \mathrm{H}) \lesssim 8.1)$ host PAH populations with small sizes on average. We do not have the long-wavelength data to sufficient depth or spatial resolution for a $\mathrm{I}_{17} / \mathrm{I}_{11.3}$ analysis; however, that data is available in the Sandstrom et al. [174] maps, and they determine that the $\mathrm{N}_{C}^{\min }$ value is smaller in the SMC than in the Milky Way based on the weak $17.0 \mu \mathrm{m}$ PAH feature. Our own results, including a wide range of $\mathrm{I}_{8.6} / \mathrm{I}_{6.2}$ ratio values, suggest that these average trends do not hold on small size scales, where local effects likely come in to play.

\subsubsection{N66 PAH Emission in Context}

In this section, we compare the PAH and forbidden line emission for N66 with data from other sources with similar star formation environments to N66, including giant HiI regions and blue compact dwarf galaxies (BCDs). For the PAH emission, we take the ratio of the $11.3 \mu \mathrm{m}$ and $6.2 \mu \mathrm{m}$ PAH features which will trace the ionization state of the PAHs and also the PAH size distribution. For the gas, 


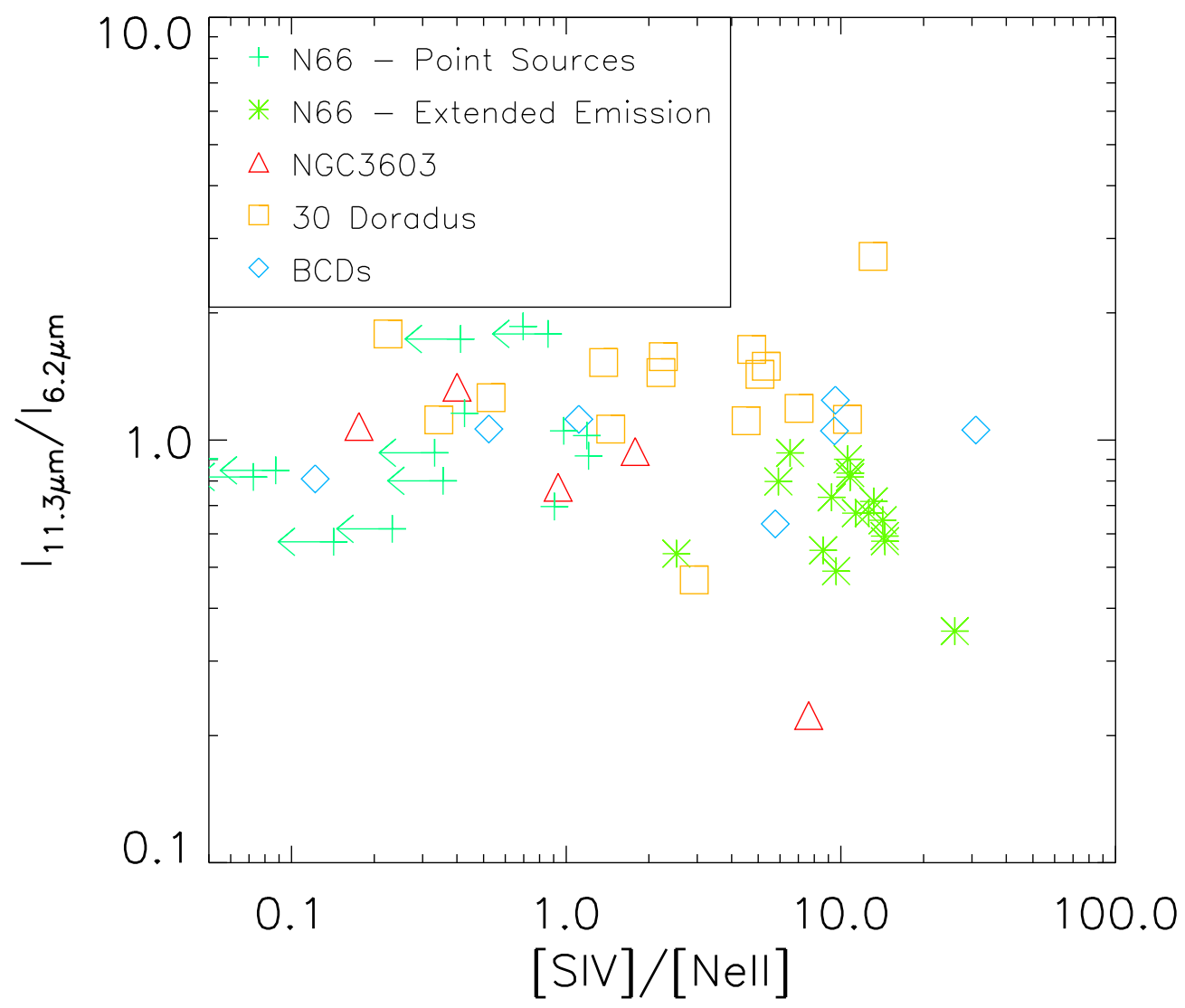

Figure 4.14: The PAH ratio $\mathrm{I}_{11.3} / \mathrm{I}_{6.2}$ versus $[\mathrm{Siv} /[\mathrm{NeII}]$ for $\mathrm{N} 66$ point sources (plus signs) and extended emission spectra (stars) plotted along with results for NGC 3603 (triangles), 30 Doradus (squares), and some well-studied BCDs (diamonds). References for these data are given in the text.

we take the $[\mathrm{SIV}] /[\mathrm{NeII}]$ ratio as a tracer of the radiation field hardness. The other giant HiI regions studied are 30 Doradus and NGC 3603 [115, 117], and the population of well-studied BCDs comes from Wu et al. [229]. The results are plotted in Figure 4.14.

The N66 point sources have PAH ratio values similar to sources with small $[\mathrm{SIV}] /[\mathrm{NeII}]$ ratio values. However, the extended emission spectra, whose $[\mathrm{SIV}] /[\mathrm{NeII}]$ ratio is on average higher than most other sources, also exhibits a lower $\mathrm{I}_{11.3} / \mathrm{I}_{6.2}$ value, as 
was discussed in $\S 4.5 .5$, suggesting that the PAH population in the diffuse ISM of N66 is more ionized than in the other HII regions and in integrated spectra of BCDs, despite sometimes equally hard ISRFs as traced by the [SIV]/[NeII] ratio.

There is a source of bias in this comparison worth noting. The spectra of 30 Doradus, NGC 3603, and the BCD sample were not extracted using SMART-AdOpt as described in $\S$ 4.4.2. Instead, they are all composite spectra. For the giant Hiı regions NGC 3603 and 30 Doradus, each spectrum likely has contributions from both unresolved point sources in the PDR and diffuse Hir emission. For the BCDs, each spectrum likely contains emission from both star-forming and quiescent regions. It is therefore not surprising that the results for N66 are both separated and qualitatively different from those of the other regions.

\subsubsection{Fraction of PAH Emission Coming From the Point Sources}

The SMC (including N66) is the closest low-metallicity star forming galaxy to the Milky Way and can therefore be used to study possible trends to other lowmetallicity star-forming environments. It is therefore important to determine what the dominant source of the PAH emission in N66 is, in order to properly interpret unresolved HII region studies. Does the PAH emission primarily come from the high $\mathrm{S} / \mathrm{N}$ point sources, or from the diffuse ISM in the HII region and PDR? 
Using the spectral maps of N66 presented in Sandstrom et al. $[173,174]$ in order to study a spatially well-sampled dataset, we made maps of the $6.2 \mu \mathrm{m}$ and $11.3 \mu \mathrm{m}$ PAH features by subtracting away the continuum measured locally. The $7.7 \mu \mathrm{m}$ feature straddles the SL1 and SL2 orders and the $8.6 \mu \mathrm{m}$ feature is very hard to pick out from the continuum, so these two features were excluded from the analysis. The average brightness in the PDR was measured and then point sources were defined as anything that was 3- $\sigma$ above the average; the annulus selected around each point source was 6 pixels across, or 10.8". Using this metric, we summed up the emission for point sources as well as the diffuse emission from the entire map in the $6.2 \mu \mathrm{m}$ and $11.3 \mu \mathrm{m}$ PAH features. There are a total of 13 selected point sources in the $6.2 \mu \mathrm{m}$ map, and 14 in the $11.3 \mu \mathrm{m}$ map, and the map is $300 " \times 430 "$. We find that the fraction of the PAH emission coming from the point sources is $28 \pm 8 \%$ for the $6.2 \mu \mathrm{m}$ PAH feature and $21 \pm 6 \%$ for the $11.3 \mu \mathrm{m}$ PAH feature. Due to the relatively high level of variance between these two PAH features, we conclude that, as a rough proxy for studies of unresolved star formation at low-metallicity, the point sources (resolved down to $\sim 2 \mathrm{pc}$ ) contribute roughly $\sim 20-35 \%$ of the $\mathrm{PAH}$ emission across the region. This means that it is the extended emission which dominates the observed PAH brightness in N66, and possibly low-metallicity giant His regions generally. 


\subsection{CONCLUSION}

N66 is the largest starburst in the SMC and contains half of the entire galaxy's O stars. Its hard radiation field and low metallicity make it an excellent corollary to studies of BCDs and high-redshift starbursts. Our analysis of Spitzer/IRS spectra from 5-14 $\mu \mathrm{m}$ makes use of powerful optimal extraction routines that separate emission from the dense PDR's unresolved point sources and the surrounding ISM's more diffuse emission. The point sources are all associated with sites of active star formation in the region. Of special note are the spectroscopic confirmation of a massive embedded class I YSO, the detection of silicates associated with young $(\sim 3 \mathrm{Myr})$ stellar clusters, and the detection of PAHs and silicates associated with a $\mathrm{B}[\mathrm{e}]$ star that indicates it may in fact be a Herbig AeBe star. In the diffuse ISM, we find evidence that the very small grains are being photodestroyed, consistent with previous studies of N66. The PAH emission shows several interesting features. The $11.3 \mu \mathrm{m}$ PAH complex has an unexplained centroid shift for both point sources and the diffuse ISM. In general, the PAHs are best classified as type A. However, a slight widening of the $7.7 \mu \mathrm{m}$ feature in some of the spectra is either due to the $7.46 \mu \mathrm{m}$ HI $6-5$ line in the diffuse emission spectra, or else the removal of ISM contamination that reveals type B-like circumstellar features. The $6.2 \mu \mathrm{m}$ and $7.7 \mu \mathrm{m}$ PAH band fluxes in N66 do not correlate with each other, an observation that we do not believe has not been observed in other objects. Because we were able to separate out the point source and extended emission in 
the spectral slit, the conclusions we draw from the PAH ratios are detailed where those presented in Sandstrom et al. [174] are more general: we find that the PAHs are more neutral in the densest parts of the PDR (including the entire point source catalogue), and more ionized in the diffuse ISM while Sandstrom et al. determined that the PAHs were on average more neutral than in higher-metallicity galaxies. The minimum size of the PAHs appears to be independent of ionization state and therefore independent of the ratio of the UV radiation field strength to the electron density, although Sandstrom et al. find a smaller PAH population on average. We stress that, on average, our results match the work of Sandstrom et al. [174] extremely well, and that the spread in ionization state and lack of clear trend with minimum PAH size on small scales is made possible by our spectral extraction techniques. Lastly, we have determined using spectral maps of the region that only $\sim 20-35 \%$ of the PAH emission in N66 is coming from the unresolved point sources, and it is therefore dominated by the diffuse emission. This percentage suggests that studies of unresolved low-metallicity star-forming regions in nearby BCDs and starburst galaxies will also have PAH spectra dominated by the diffuse HII region and PDR, not the unresolved point sources, and the observed PAH signatures of the point source spectra are therefore not representative of the entire HII region's properties. 
Chapter 4. An In-Depth View of the Mid-Infrared Properties of Point Sources and the Diffuse ISM in the SMC Giant HII Region, N66

\subsection{The Atlas of Reduced Spectra}

The spectra used in the analysis are presented here. The reduction method for producing the point source and extended emission is discussed in $\S$ 4.4.2. 

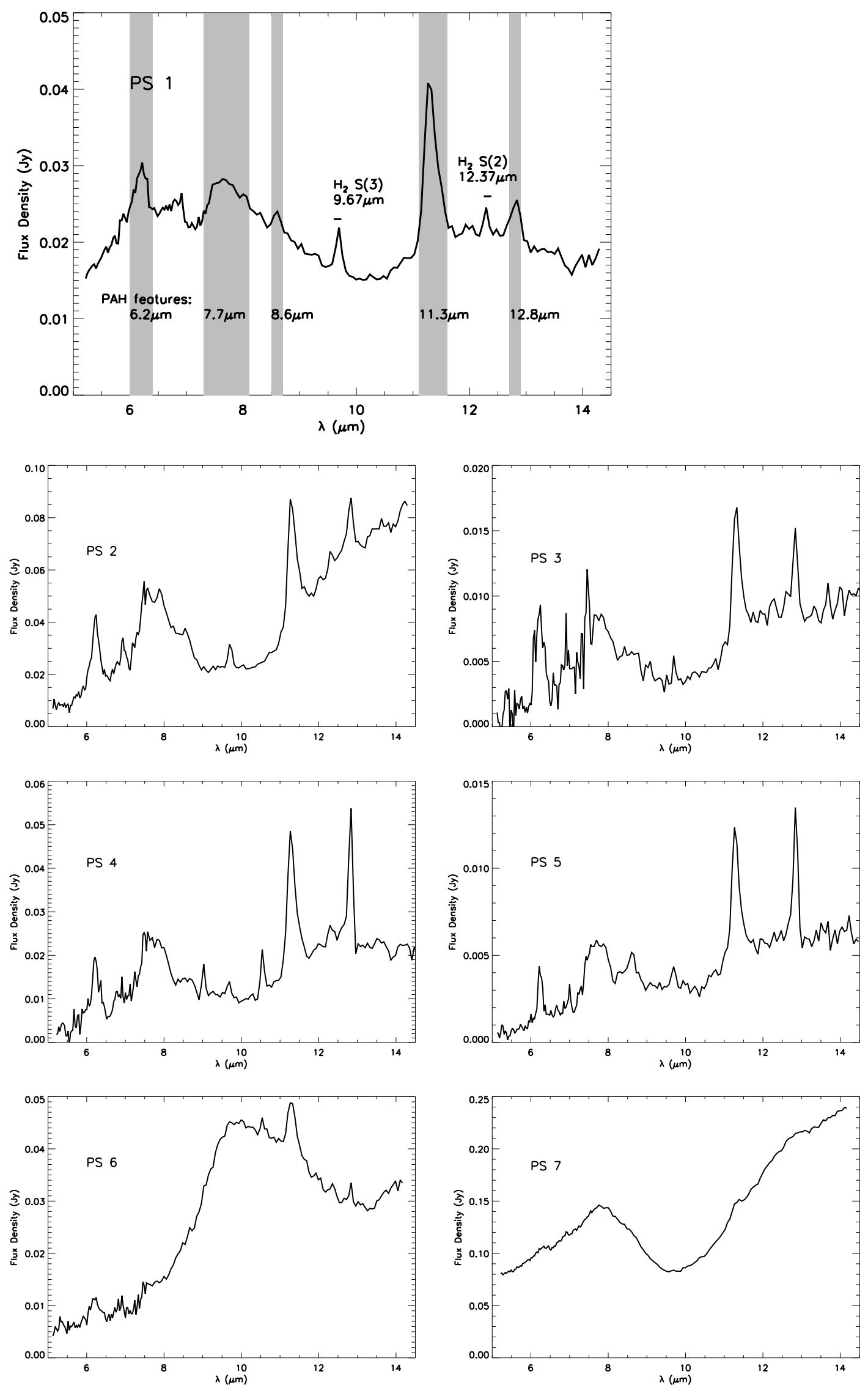

FiguRE 4.15: Spitzer spectra of point sources PS1-PS7. The spectrum of PS1 is enlarged so that the $\mathrm{PAH}$ and $\mathrm{H}_{2}$ feature labels can be seen clearly. 
Chapter 4. An In-Depth View of the Mid-Infrared Properties of Point Sources and the Diffuse ISM in the SMC Giant HII Region, N66
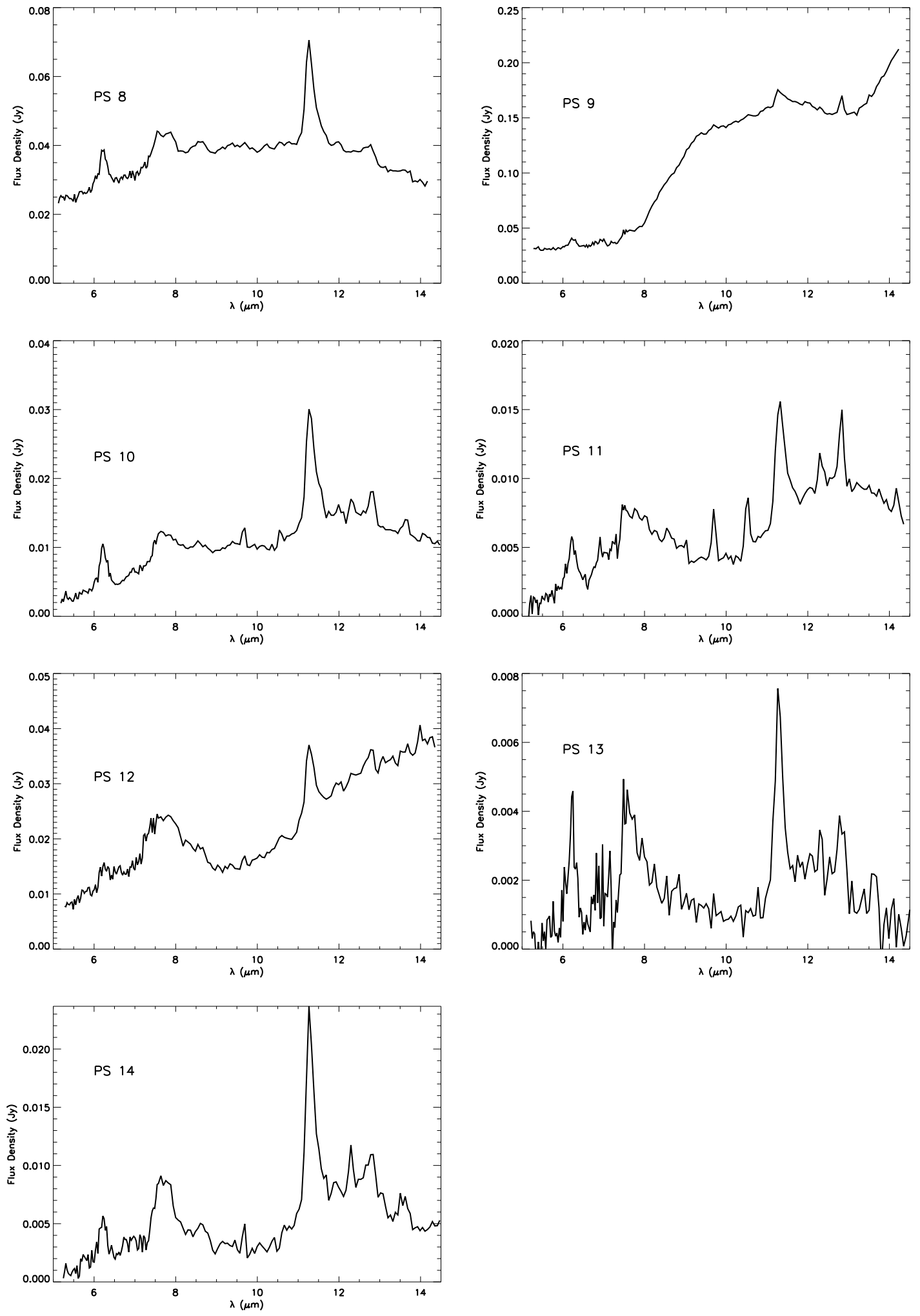

FiguRE 4.16: Spitzer spectra of point Sources PS8-PS14. 
Chapter 4. An In-Depth View of the Mid-Infrared Properties of Point Sources and the Diffuse ISM in the SMC Giant HiI Region, N66
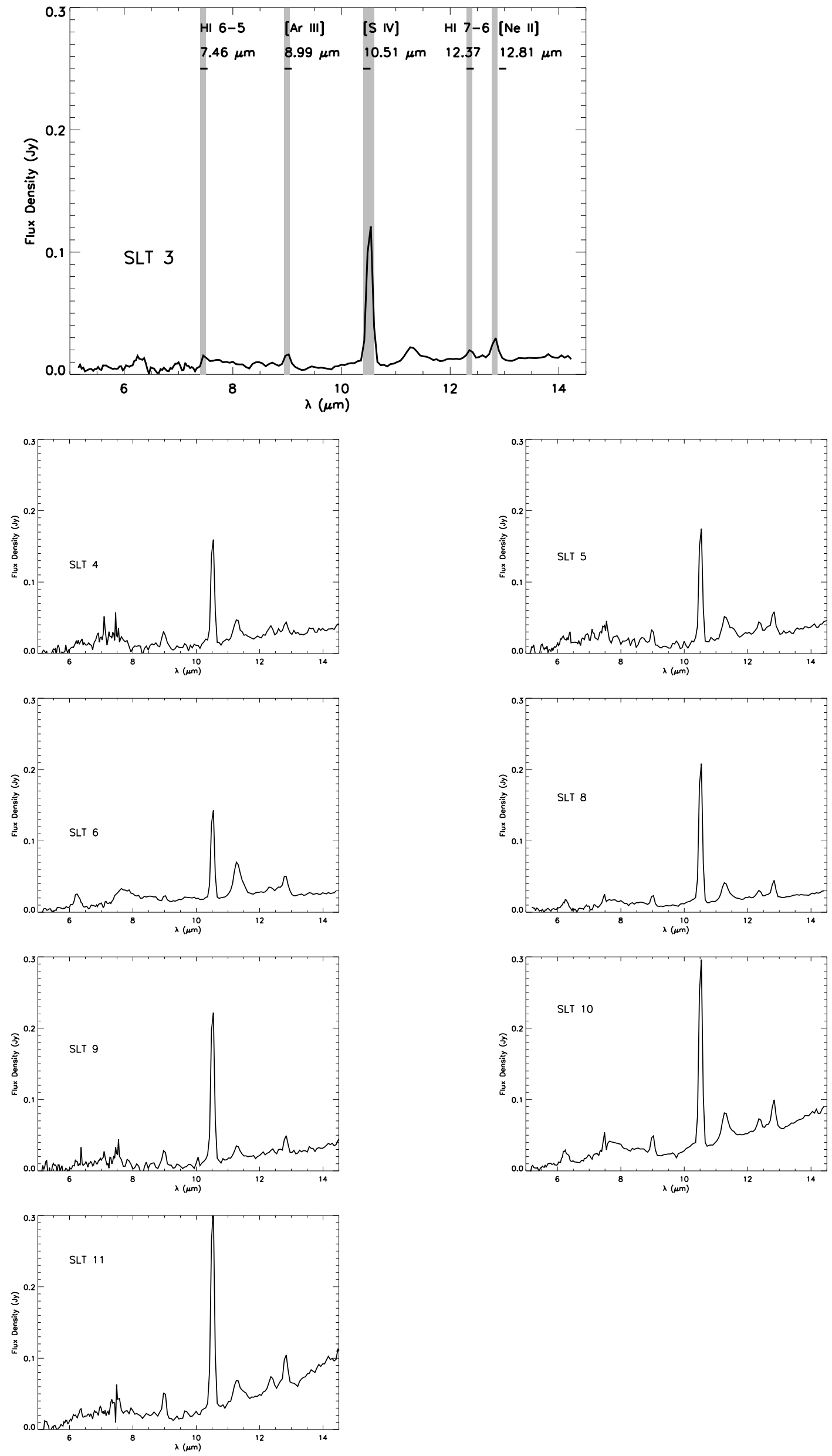

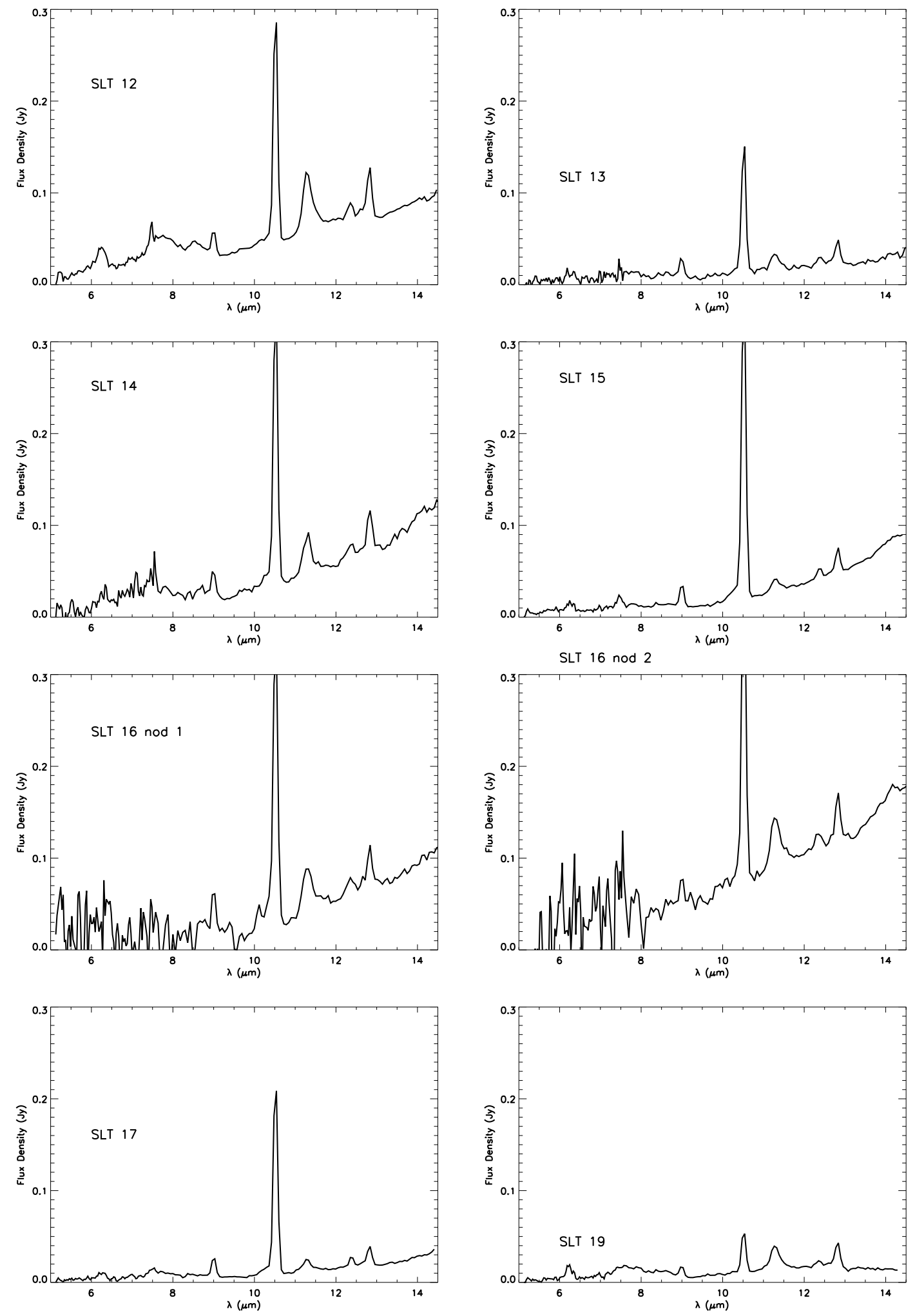

Figure 4.18: Spitzer extended emission spectra SL12-SLT19. The spectra are all plotted on the same scale, so that the relative flux in each slit can be seen. 
Chapter 4. An In-Depth View of the Mid-Infrared Properties of Point Sources and the Diffuse ISM in the SMC Giant HiI Region, N66

Table 1. Point Source Positions

RA Dec Designation

$(\mathrm{J} 2000) \quad(\mathrm{J} 2000)$

\begin{tabular}{llll}
\hline 00h59m21.8s & $-72 \mathrm{~d} 11 \mathrm{~m} 13.3 \mathrm{~s}$ & PS1 & \\
00h59m20.0s & $-72 \mathrm{~d} 11 \mathrm{~m} 21.0 \mathrm{~s}$ & PS2 & \\
00h59m17.1s & $-72 \mathrm{~d} 11 \mathrm{~m} 24.3 \mathrm{~s}$ & PS3 & \\
00h59m14.8s & $-72 \mathrm{~d} 11 \mathrm{~m} 03.8 \mathrm{~s}$ & PS4 & 1 class I YSO \\
00h59m14.0s & $-72 \mathrm{~d} 09 \mathrm{~m} 27.1 \mathrm{~s}$ & PS5 & N66C; 1 class II YSO \\
00h59m12.3s & $-72 \mathrm{~d} 09 \mathrm{~m} 58.7 \mathrm{~s}$ & PS6 & N66B; 1 class I YSO \\
00h59m09.3s & $-72 \mathrm{~d} 10 \mathrm{~m} 57.8 \mathrm{~s}$ & PS7 & N66A; 2 class I YSOs \\
00h59m05.8s & $-72 \mathrm{~d} 11 \mathrm{~m} 27.5 \mathrm{~s}$ & PS8 & KWBBE 200, a Be star \\
00h59m05.4s & $-72 \mathrm{~d} 10 \mathrm{~m} 36.1 \mathrm{~s}$ & PS9 & N66; NGC 346 \\
00h58m59.8s & $-72 \mathrm{~d} 10 \mathrm{~m} 16.7 \mathrm{~s}$ & PS10 & \\
$000 \mathrm{~h} 58 \mathrm{~m} 57.6 \mathrm{~s}$ & $-72 \mathrm{~d} 09 \mathrm{~m} 56.7 \mathrm{~s}$ & PS11 & \\
$00 \mathrm{~h} 58 \mathrm{~m} 57.0 \mathrm{~s}$ & $-72 \mathrm{~d} 09 \mathrm{~m} 54.5 \mathrm{~s}$ & PS12 & \\
\hline $00 \mathrm{~h} 58 \mathrm{~m} 54.7 \mathrm{~s}$ & $-72 \mathrm{~d} 09 \mathrm{~m} 51.5 \mathrm{~s}$ & PS13 & \\
\hline & $-72 \mathrm{~d} 09 \mathrm{~m} 29.2 \mathrm{~s}$ & PS14 & \\
\hline
\end{tabular}


Chapter 4. An In-Depth View of the Mid-Infrared Properties of Point Sources and the Diffuse ISM in the SMC Giant HII Region, N66

Table 2. Slit Positions

\begin{tabular}{|c|c|c|c|}
\hline RA & Dec & Designation & Notes \\
\hline $00 \mathrm{~h} 59 \mathrm{~m} 49.99 \mathrm{~s}$ & $-72 \mathrm{~d} 13 \mathrm{~m} 00.1 \mathrm{~s}$ & SLT1 & sky position \\
\hline $00 \mathrm{~h} 59 \mathrm{~m} 42.00 \mathrm{~s}$ & $-72 \mathrm{~d} 11 \mathrm{~m} 09.9 \mathrm{~s}$ & SLT2 & sky position \\
\hline 00h59m21.62s & $-72 \mathrm{~d} 11 \mathrm{~m} 17.2 \mathrm{~s}$ & SLT3 & \\
\hline $00 \mathrm{~h} 59 \mathrm{~m} 20.42 \mathrm{~s}$ & $-72 \mathrm{~d} 11 \mathrm{~m} 22.2 \mathrm{~s}$ & SLT4 & \\
\hline $00 \mathrm{~h} 59 \mathrm{~m} 17.30 \mathrm{~s}$ & $-72 \mathrm{~d} 11 \mathrm{~m} 25.1 \mathrm{~s}$ & SLT5 & \\
\hline $00 \mathrm{~h} 59 \mathrm{~m} 15.26 \mathrm{~s}$ & $-72 \mathrm{~d} 09 \mathrm{~m} 19.1 \mathrm{~s}$ & SLT6 & \\
\hline $00 \mathrm{~h} 59 \mathrm{~m} 14.69 \mathrm{~s}$ & $-72 \mathrm{~d} 11 \mathrm{~m} 03.1 \mathrm{~s}$ & SLT7 & \\
\hline $00 \mathrm{~h} 59 \mathrm{~m} 13.49 \mathrm{~s}$ & $-72 \mathrm{~d} 09 \mathrm{~m} 54.7 \mathrm{~s}$ & SLT8 & \\
\hline $00 \mathrm{~h} 59 \mathrm{~m} 12.29 \mathrm{~s}$ & $-72 \mathrm{~d} 09 \mathrm{~m} 58.3 \mathrm{~s}$ & SLT9 & \\
\hline 00h59m10.13s & $-72 \mathrm{~d} 10 \mathrm{~m} 51.2 \mathrm{~s}$ & SLT10 & \\
\hline $00 \mathrm{~h} 59 \mathrm{~m} 09.24 \mathrm{~s}$ & $-72 \mathrm{~d} 10 \mathrm{~m} 57.0 \mathrm{~s}$ & SLT11 & \\
\hline $00 \mathrm{~h} 59 \mathrm{~m} 06.74 \mathrm{~s}$ & $-72 \mathrm{~d} 10 \mathrm{~m} 25.3 \mathrm{~s}$ & SLT12 & \\
\hline 00h59m05.98s & $-72 \mathrm{~d} 11 \mathrm{~m} 26.9 \mathrm{~s}$ & SLT13 & \\
\hline $00 \mathrm{~h} 59 \mathrm{~m} 05.52 \mathrm{~s}$ & $-72 \mathrm{~d} 10 \mathrm{~m} 35.8 \mathrm{~s}$ & SLT14 & \\
\hline $00 \mathrm{~h} 58 \mathrm{~m} 59.11 \mathrm{~s}$ & $-72 \mathrm{~d} 10 \mathrm{~m} 23.2 \mathrm{~s}$ & SLT15 & \\
\hline $00 \mathrm{~h} 58 \mathrm{~m} 59.02 \mathrm{~s}$ & $-72 \mathrm{~d} 10 \mathrm{~m} 28.6 \mathrm{~s}$ & SLT16 & \\
\hline $00 \mathrm{~h} 58 \mathrm{~m} 58.32 \mathrm{~s}$ & $-72 \mathrm{~d} 09 \mathrm{~m} 50.0 \mathrm{~s}$ & SLT17 & \\
\hline $00 \mathrm{~h} 58 \mathrm{~m} 56.95 \mathrm{~s}$ & $-72 \mathrm{~d} 09 \mathrm{~m} 54.0 \mathrm{~s}$ & SLT18 & \\
\hline $00 \mathrm{~h} 58 \mathrm{~m} 52.42 \mathrm{~s}$ & $-72 \mathrm{~d} 09 \mathrm{~m} 24.1 \mathrm{~s}$ & SLT19 & \\
\hline
\end{tabular}


Table 3. Atomic, Ionic, and Molecular Hydrogen Line Measurements

\begin{tabular}{|c|c|c|c|c|c|c|c|}
\hline Spectrum & HI 6-5 & [ArIII] & $\mathrm{H}_{2} \mathrm{~S}(3)$ & {$[\mathrm{SIV}]$} & $\mathrm{H}_{2} \mathrm{~S}(2)$ & HI 7-6 & [NeII] \\
\hline \multirow[t]{2}{*}{ - } & $7.46 \mu \mathrm{m}$ & $8.99 \mu \mathrm{m}$ & $9.67 \mu \mathrm{m}$ & $10.51 \mu \mathrm{m}$ & $12.28 \mu \mathrm{m}$ & $12.37 \mu \mathrm{m}$ & $12.81 \mu \mathrm{m}$ \\
\hline & \multicolumn{7}{|c|}{$\times 10^{-21} \mathrm{~W} \mathrm{~cm}^{-2}$} \\
\hline PS1 & $<0.47$ & $<0.52$ & $2.2 \pm 0.3$ & $<0.11$ & $0.77 \pm 0.12$ & $<0.22$ & $1.5 \pm 0.3$ \\
\hline $\mathrm{PS} 2$ & $3.3 \pm 0.7$ & $<0.47$ & $3.8 \pm 0.5$ & $<0.41$ & $1.6 \pm 0.2$ & $<0.12$ & $4.7 \pm 0.8$ \\
\hline PS3 & $0.8 \pm 0.2$ & $<0.32$ & $0.64 \pm 0.12$ & $<0.18$ & $0.41 \pm 0.08$ & $<0.12$ & $1.3 \pm 0.3$ \\
\hline PS4 & $<0.80$ & $1.6 \pm 0.4$ & $1.3 \pm 0.2$ & $2.6 \pm 0.5$ & $<0.91$ & $<0.30$ & $6.2 \pm 1.0$ \\
\hline PS5 & $<0.05$ & $<0.09$ & $5.4 \pm 1.1$ & $<0.09$ & $<0.056$ & $<0.15$ & $1.9 \pm 0.4$ \\
\hline PS6 & $<0.29$ & $<0.09$ & $<0.38$ & $0.39 \pm 0.10$ & $<0.097$ & $<0.30$ & $0.55 \pm 0.13$ \\
\hline PS7 & $<1.0$ & $<0.80$ & $0.54 \pm 0.11$ & $<0.68$ & $<0.52$ & $<0.38$ & $<0.70$ \\
\hline PS8 & $<0.40$ & $<0.42$ & $<0.59$ & $<0.45$ & $<0.11$ & $<0.10$ & $<0.38$ \\
\hline PS9 & $<0.67$ & $<0.85$ & $<2.1$ & $<0.81$ & $<0.68$ & $<0.68$ & $3.5 \pm 0.6$ \\
\hline PS10 & $<0.62$ & $<0.17$ & $1.3 \pm 0.2$ & $<0.42$ & $0.54 \pm 0.9$ & $<0.052$ & $1.3 \pm 0.3$ \\
\hline PS11 & $<0.45$ & $<0.05$ & $1.4 \pm 0.2$ & $1.5 \pm 0.3$ & $0.46 \pm 0.08$ & $0.31 \pm 0.09$ & $1.3 \pm 0.3$ \\
\hline PS12 & $<0.45$ & $<0.38$ & $0.67 \pm 0.13$ & $<0.39$ & $0.29 \pm 0.06$ & $<0.032$ & $0.45 \pm 0.11$ \\
\hline PS13 & $<0.37$ & $<0.33$ & $<0.37$ & $<0.14$ & $<0.35$ & $<0.35$ & $0.44 \pm 0.10$ \\
\hline PS14 & $<0.15$ & $<0.21$ & $1.2 \pm 0.2$ & $<0.40$ & $0.89 \pm 0.14$ & $<0.037$ & $0.98 \pm 0.21$ \\
\hline
\end{tabular}


Table 4. Atomic, Ionic, and Molecular Hydrogen Line Measurements

\begin{tabular}{|c|c|c|c|c|c|c|c|}
\hline \multirow[t]{2}{*}{ Spectrum } & HI 6-5 & [ArIII] & $\mathrm{H}_{2} \mathrm{~S}(3)$ & {$[\mathrm{SIV}]$} & $\mathrm{H}_{2} \mathrm{~S}(2)$ & HI 7-6 & {$[\mathrm{NeII}]$} \\
\hline & $7.46 \mu \mathrm{m}$ & $8.99 \mu \mathrm{m}$ & $9.67 \mu \mathrm{m}$ & $10.51 \mu \mathrm{m}$ & $12.28 \mu \mathrm{m}$ & $12.37 \mu \mathrm{m}$ & $12.81 \mu \mathrm{m}$ \\
\hline- & \multicolumn{7}{|c|}{$\times 10^{-21} \mathrm{~W} \mathrm{~cm}^{-2}$} \\
\hline SLT3 & $<4.0$ & $4.3 \pm 0.9$ & $<0.52$ & $41 \pm 5$ & $<0.39$ & $1.7 \pm 0.4$ & $4.2 \pm 0.8$ \\
\hline SLT4 & $<2.9$ & $1.2 \pm 2$ & $<1.2$ & $55 \pm 6$ & $<0.79$ & $3.7 \pm 0.7$ & $4.2 \pm 0.7$ \\
\hline SLT5 & $<3.9$ & $8.3 \pm 1.7$ & $<4.7$ & $60 \pm 0.7$ & $<1.3$ & $3.5 \pm 0.7$ & $5.3 \pm 0.9$ \\
\hline SLT6 & $2.0 \pm 0.5$ & $3.5 \pm 0.8$ & $<0.26$ & $54 \pm 6$ & $<1.6$ & $0.60 \pm 0.16$ & $9.1 \pm 1.4$ \\
\hline SLT8 & $4.5 \pm 0.9$ & $7.0 \pm 1.4$ & $<0.57$ & $72 \pm 8$ & $<0.31$ & $2.6 \pm 0.5$ & $5.7 \pm 1.0$ \\
\hline SLT9 & $<0.82$ & $9.6 \pm 1.9$ & $<0.77$ & $79 \pm 9$ & $<0.82$ & $<1.0$ & $5.5 \pm 0.9$ \\
\hline SLT10 & $7.0 \pm 1.4$ & $13 \pm 2$ & $<0.40$ & $94 \pm 10$ & $<0.46$ & $4.2 \pm 0.8$ & $8.7 \pm 1.4$ \\
\hline SLT11 & $<2.3$ & $19 \pm 3$ & $<1.4$ & $104 \pm 11$ & $<0.38$ & $6.3 \pm 1.2$ & $11 \pm 2$ \\
\hline SLT12 & $8.9 \pm 1.6$ & $11 \pm 2$ & $<0.38$ & $87 \pm 9$ & $<0.60$ & $4.0 \pm 0.8$ & $13 \pm 2$ \\
\hline SLT13 & $<8.3$ & $10 \pm 2$ & $<0.67$ & $50 \pm 6$ & $<0.19$ & $2.7 \pm 0.6$ & $5.8 \pm 1.0$ \\
\hline SLT14 & $<3.9$ & $11 \pm 2$ & $<0.32$ & $106 \pm 11$ & $1.3 \pm 0.2$ & $2.6 \pm 0.5$ & $9.8 \pm 1.5$ \\
\hline SLT15 & $8.4 \pm 1.6$ & $11 \pm 2$ & $<0.28$ & $153 \pm 15$ & $<0.27$ & $2.7 \pm 0.6$ & $5.9 \pm 1.0$ \\
\hline SLT16 nod 1 & $<11$ & $18 \pm 3$ & $<1.8$ & $116 \pm 12$ & $<1.3$ & $6.4 \pm 1.2$ & $8.0 \pm 1.3$ \\
\hline SLT16 nod 2 & $<19$ & $11 \pm 2$ & $<3.5$ & $123 \pm 13$ & $2.7 \pm 0.3$ & $2.6 \pm 0.6$ & $12 \pm 2$ \\
\hline SLT17 & $<0.40$ & $9.6 \pm 1.9$ & $<0.19$ & $73 \pm 8$ & $<0.27$ & $2.5 \pm 0.5$ & $5.2 \pm 0.9$ \\
\hline SLT19 & $<0.24$ & $5.1 \pm 1.1$ & $<0.10$ & $16 \pm 2$ & $<0.23$ & $1.7 \pm 0.4$ & $6.3 \pm 1.1$ \\
\hline
\end{tabular}


Chapter 4. An In-Depth View of the Mid-Infrared Properties of Point Sources and the Diffuse ISM in the SMC Giant HiI Region, N66

Table 5. PAH and Continuum Measurements

\begin{tabular}{|c|c|c|c|c|c|}
\hline Spectrum & $\mathrm{PAH}_{6.2 \mu m}$ & $\begin{array}{r}\mathrm{PAH}_{7.7 \mu m} \\
\times 10^{-20}\end{array}$ & 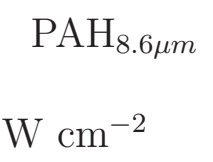 & $\mathrm{PAH}_{11.3 \mu m}$ & $\begin{array}{l}14 \mu \mathrm{m} \text { continuum } \\
\times 10^{-20} \mathrm{~W} \mathrm{~cm}^{-2} \mu \mathrm{m}^{-1}\end{array}$ \\
\hline PS1 & $2.9 \pm 0.1$ & $2.4 \pm 0.27$ & $0.67 \pm 0.15$ & $2.38 \pm 0.07$ & 1.6 \\
\hline PS2 & $7.4 \pm 0.3$ & $18 \pm 2$ & $3.5 \pm 0.5$ & $6.2 \pm 0.4$ & 5.9 \\
\hline PS3 & $2.1 \pm 0.2$ & $4.9 \pm 0.3$ & $0.89 \pm 0.22$ & $1.2 \pm 0.2$ & 0.98 \\
\hline PS4 & $2.9 \pm 0.2$ & $7.4 \pm 1.3$ & $1.4 \pm 0.4$ & $3.3 \pm 0.3$ & 2.1 \\
\hline PS5 & $0.71 \pm 0.03$ & $2.10 \pm 0.9$ & $0.71 \pm 0.03$ & $0.64 \pm 0.02$ & 0.68 \\
\hline PS6 & $0.28 \pm 0.02$ & $0.96 \pm 0.04$ & $0.21 \pm 0.05$ & $0.52 \pm 0.01$ & 0.65 \\
\hline PS7 & $1.6 \pm 0.3$ & $11 \pm 1$ & $0.99 \pm 0.43$ & $1.7 \pm 0.1$ & 18 \\
\hline PS8 & $2.5 \pm 0.4$ & $6.3 \pm 1.1$ & $1.2 \pm 0.3$ & $2.26 \pm 0.06$ & 3.0 \\
\hline PS9 & $2.8 \pm 0.5$ & $0.05 \pm 0.06$ & $2.8 \pm 0.5$ & $1.7 \pm 0.1$ & 16 \\
\hline PS10 & $1.61 \pm 0.05$ & $3.2 \pm 0.3$ & $0.59 \pm 0.08$ & $1.50 \pm 0.03$ & 1.2 \\
\hline PS11 & $0.99 \pm 0.05$ & $2.6 \pm 0.2$ & $0.54 \pm 0.06$ & $1.01 \pm 0.03$ & 0.85 \\
\hline PS12 & $0.81 \pm 0.08$ & $8.1 \pm 0.7$ & $1.0 \pm 0.2$ & $1.45 \pm 0.07$ & 3.5 \\
\hline PS13 & $0.62 \pm 0.02$ & $1.67 \pm 0.07$ & $0.25 \pm 0.04$ & $0.50 \pm 0.01$ & 0.11 \\
\hline PS14 & $1.20 \pm 0.07$ & $3.5 \pm 0.2$ & $0.48 \pm 0.08$ & $2.1 \pm 0.1$ & 0.60 \\
\hline
\end{tabular}


Chapter 4. An In-Depth View of the Mid-Infrared Properties of Point Sources and the Diffuse ISM in the SMC Giant HII Region, N66

Table 6. PAH and Continuum Measurements

Spectrum $\quad \mathrm{PAH}_{6.2 \mu m} \quad \mathrm{PAH}_{7.7 \mu m} \quad \mathrm{PAH}_{8.6 \mu m} \quad \mathrm{PAH}_{11.3 \mu m} \quad 14 \mu \mathrm{m}$ continuum

\begin{tabular}{|c|c|c|c|c|c|}
\hline & & $\times 10^{-20}$ & $\mathrm{~W} \mathrm{~cm}^{-2}$ & & $\times 10^{-20} \mathrm{~W} \mathrm{~cm}^{-2} \mu \mathrm{m}^{-1}$ \\
\hline SLT3 & $2.6 \pm 0.3$ & $3.4 \pm 0.34$ & $1.1 \pm 0.2$ & $1.3 \pm 0.3$ & 2.2 \\
\hline SLT4 & $3.1 \pm 0.31$ & $18.6 \pm 0.9$ & $0.81 \pm 0.30$ & $2.2 \pm 0.2$ & 4.8 \\
\hline SLT5 & $4.3 \pm 0.4$ & $17 \pm 2$ & $1.9 \pm 0.6$ & $2.9 \pm 0.4$ & 5.3 \\
\hline SLT6 & $5.2 \pm 0.3$ & $11.4 \pm 0.8$ & $3.3 \pm 0.3$ & $4.2 \pm 0.3$ & 4.3 \\
\hline SLT8 & $3.7 \pm 0.3$ & $7.3 \pm 0.56$ & $1.9 \pm 0.4$ & $2.5 \pm 0.5$ & 3.8 \\
\hline SLT9 & $2.4 \pm 0.4$ & $10.1 \pm 0.9$ & $0.51 \pm 0.35$ & $1.4 \pm 0.2$ & 4.9 \\
\hline SLT10 & $6.7 \pm 0.4$ & $19 \pm 1$ & $2.6 \pm 0.8$ & $5.5 \pm 0.6$ & 8.6 \\
\hline SLT11 & $3.5 \pm 0.3$ & $19 \pm 1$ & $1.7 \pm 0.5$ & $2.5 \pm 0.2$ & 9.7 \\
\hline SLT12 & $8.0 \pm 0.5$ & $17 \pm 2$ & $3.0 \pm 0.8$ & $7.4 \pm 0.7$ & 9.1 \\
\hline SLT13 & $2.3 \pm 0.3$ & $5.2 \pm 0.7$ & $1.1 \pm 0.3$ & $1.3 \pm 0.1$ & 4.4 \\
\hline SLT14 & $4.0 \pm 0.5$ & $21 \pm 2$ & $1.9 \pm 0.6$ & $3.3 \pm 0.3$ & 7.9 \\
\hline SLT15 & $3.0 \pm 0.2$ & $6.9 \pm 0.69$ & $1.2 \pm 0.4$ & $1.0 \pm 0.2$ & 8.6 \\
\hline SLT16 nod 1 & $6.8 \pm 2.2$ & $1.9 \pm 2.4$ & $2.2 \pm 1.8$ & $4.0 \pm 1.0$ & 9.6 \\
\hline SLT16 nod 2 & $4.9 \pm 2.0$ & $9.6 \pm 4.0$ & $2.3 \pm 1.9$ & $4.4 \pm 1.0$ & 12 \\
\hline SLT17 & $1.9 \pm 0.1$ & $4.6 \pm 0.3$ & $0.60 \pm 0.14$ & $1.20 \pm 0.07$ & 1.7 \\
\hline SLT19 & $3.8 \pm 1.0$ & $9.5 \pm 0.3$ & $2.6 \pm 0.2$ & $2.02 \pm 0.06$ & 2.3 \\
\hline
\end{tabular}




\section{Chapter 5}

\section{Investigating Hydrogen}

\section{Recombination Theory in HII}

\section{Regions Around Starbursts}

\subsection{ABSTRACT}

Many modern line radiative transfer codes utilize model Hydrogen atoms that allow for detailed studies of gaseous nebulae beyond Case B recombination theory. However, the contribution of Hydrogen line pumping and fluorescence has been tackled only on theoretical grounds, and implementation in realistic H II region models is limited. In this chapter, I study how source geometry and input spectral resolution affect model line fluxes and ratios. In the case of constant-density 
models, using appropriately high wavlength-resolution input spectra yield realistic contributions of pumping and fluorescence to the Hydrogen lines, producing an extra $\sim 13 \%$ line flux in $\mathrm{H} \beta$ and $<4 \%$ in $\mathrm{H} \alpha / \mathrm{H} \beta$ line ratio. When using input spectra with low wavelength resolution, pumping and fluorescence contributions to the line fluxes are significantly over-produced (an additional 70-90\% $\mathrm{H} \beta$ line flux and $\sim 9 \%$ in $\mathrm{H} \alpha / \mathrm{H} \beta$ ratio). "Blister" $\mathrm{H}$ II region model geometries do not suffer nearly as much from pumping and fluorescence $(<2 \%$ in $\mathrm{H} \beta$ and $\mathrm{H} \alpha / \mathrm{H} \beta$ ratio). I implement grids of models to illustrate the magnitude of this effect in a wide variety of parameter space. These models illustrate the dangers of using simplified geometries and low-resolution ionizing spectra with modern codes, and suggest that, in spite of the sophistication of many codes, assuming Case B recombination theory and a blister H II region geometry is in many cases the most robust solution.

\subsection{INTRODUCTION: Menzel's Cases and Mod- ern Models}

Between 1937 and 1939, Donald Menzer, James Baker, and collaborators undertook a project to formulate physical processes in astrophysical gaseous nebulae. The most highly cited paper in the series of published results is Baker \& Menzel [6], in which they describe two "Cases" that are optical depth limits on the Hydrogen emission from observable ionized (i.e. radiation bounded) gaseous nebulae. 
The first, Case A, imagines an ionized nebula that is optically thin in all Hi recombination lines, thereby assuming that all of the Lyman series emitted photons escape the nebula. Another noteworthy assumption of Case A is that the excited levels are only populated by radiative recombination. The second scenario, Case B, imagines an optically thick nebula, in which the Lyman emission lines are all optically thick and must scatter in the nebula multiple times before being emitted in Balmer, Paschen, etc. series photons, and then finally Lyman- $\alpha$ (Ly $\alpha$ ) or as a 2-photon continuum. Any emitted Lyman- $\beta(\operatorname{Ly} \beta)$ photons are supposed to be absorbed and re-emitted as Balmer- $\alpha(\mathrm{H} \alpha)$, and therefore cannot escape from the nebula. Corrections to Baker \& Menzel's first calculations and radiative recombination rates were presented by Seaton [180, 181].

In comparisons to astrophysical nebulae, it was quickly discovered that Case A required such thin clouds as to be practically unobservable [e.g. 182], and collisions were determined to be substantially less important than spontaneous recombination rates for Hydrogen line measurements [20, 154]. Case B has been relied on, therefore, as the benchmark for understanding the Hydrogen emission from all gaseous nebulae. This includes stellar photospheres, HiI regions, planetary nebulae, and the diffuse interstellar medium (ISM) in the Milky Way, as well as entire galaxies - from local spirals and dwarfs to high-redshift Lyman- $\alpha$ emitters. In two of the most highly-cited examples of the application of Case B recombination theory, Hummer \& Storey [85] and Storey \& Hummer [197] published the 
relative intensities of Hi and HeII lines from levels 1 to 50 and 1 to 1000 (respective to the two papers), including collisional effects, thereby setting a benchmark against which most observations of gaseous nebulae could readily be compared. As a comparison to real nebulae, Case B is always an upper limit on the observed emissivities [as discussed in 61], although a substantial fraction of modern considerations do not make mention of this fact $[e . g .22,137]$.

As part of their original work, Baker et al. [7] also considered a third limit, called Case C, in which the Lyman lines are optically thin, and photoionized Hydrogen is continuously pumped by the ionizing continuum followed by fluorescence through the Lyman series, whose photons have a definite escape probability. Case $\mathrm{C}$ has not received much attention, for two reasons: (1) stellar photospheres, the first application of Hydrogen recombination theory, are well-explained by Case B; and (2) Case B was re-affirmed as a very good approximation for HiI region and planetary nebula observations [e.g. 23, 182].

What makes Case $\mathrm{C}$ pertinent to astrophysical nebulae and worth investigating further is the fact that Lyman line fluorescence should create higher emissivities than Case B, i.e. Case B is not the emissivity upper limit that is often assumed. This was investigated in detail by Chamberlain [28], but was not discussed again seriously in the literature until Ferland [61], when Case C recombination theory was made available in the CLOUDY90 gaseous nebula code [62]. Ferland [61] produced the first predictions of Hydrogen line intensities and ratios as a function 
of optical depth and source temperature, and further predicted that Case C would be a useful description of nebulae with optical depths of $\tau_{V} \sim 1 / 100$.

Luridiana et al. [124] continued the trend of thought begun by Baker et al., by christening a "Case D", which is useful for optically thick nebulae that allow continuum pumping and fluorescence in its Lyman and Balmer lines, and therefore stands as an additive correction to Case B. The Cases C/D are fully allowed in the CLOUDY code, version 10.00.

What CLOUDY allows us to do is to use a model Hydrogen atom and computed line optical depths in order to determine escape probabilities for an irradiated nebula. CLOUDY can then output computed line intensities and emergent fluxes with which to compare to data of astrophysical systems. In Yeh et al. [232], Verdolini et al. [214], CLOUDY was used in this way, to study metal and Hydrogen lines in constant-pressure (i.e. static) and constant density H II regions. Their results are not as important to the conclusions of this chapter as some of their assumptions, which will be discussed below.

In this chapter, I make a detailed study of the effects of geometry and the input spectrum's resolution on output Hydrogen line fluxes in typical H II regions. I briefly describe the full Hydrogen atom model that is implemented in CLOUDY in Section 5.3, discuss how a realistic H II region is made with CLOUDY in Section 5.4, illustrate the pitfalls of not modeling realistic H II regions in Section 5.5, and then conclude in Section 5.6. 


\subsection{The CLOUDY Code's Model Hydrogen Atom}

The CLOUDY Hydrogen atom is described in Ferguson \& Ferland [59], but I present some of its most important features here. Whereas other Hydrogen models [e.g. 85] are computationally intensive because they solve for all radiative and collisional processes, CLOUDY is distinguished by its efficiency, made possible by utilizing many results from previous works, but is still able to re-produce the recombination efficiency and line spectrum of larger codes. For example, whereas Storey \& Hummer [197] calculates a complete hydrogen emission spectrum for 1000 levels of the Hydrogen atom, Ferguson \& Ferland [59] does with only a few dozen levels. Ferguson \& Ferland find that the limit to their accuracy is not the number of levels used for the Hydrogen atom, but the collisional rates.

Ferguson \& Ferland's Hydrogen atom has independent $1 s, 2 s, 2 p$, and an arbitrary number of $\mathrm{n}$ higher levels. They use the Milne relation to determine radiative recombination rate coefficients up to $n=15$, and an asymptotic formula above 15. Errors on both radiative and collisional recombination are shown to be very low; less than $5 \%$ in all cases, and less than $1 \%$ in most. Collisions that change the level of the Hydrogen atom are based on well-determined answers from the literature.

The largest difficulty in constructing their atom was to include the radiative cascades required of Cases $\mathrm{C} / \mathrm{D}$. The main problem is that captured electrons tend to "bunch up" at higher $l$ angular momentum values because $\Delta l$ must equal 1 for 
allowed transitions. Collisions with slow-moving protons distribute the electrons according to more statistical weights, so there is a density dependence. To account for this density dependence, each Hydrogen transition is subject to a branching ratio that is parametrized by two variables; Ferguson \& Ferland gives these branching ratio variables and their density limits in their Table 2. Errors between their parametrization and more exact solutions of Storey \& Hummer [197] are less than $1 \%$ in all cases that were considered.

Ferguson \& Ferland is able to show that their model can reproduce the more exact results for Case B of Storey \& Hummer [197], but also shows how continuum pumping and fluorescence can affect the results. This is the primary motivation for considering Case C in Ferland [61].

\subsection{Models with High-Resolution Input Spectra}

Luridiana et al. [124] showed that spectral resolution is an essential model parameter that must be taken into account when creating numerical models of $\mathrm{H}$ II regions. For well-studied Balmer lines such as $\mathrm{H} \alpha \lambda 6563$ and $\mathrm{H} \beta \lambda 4861$, implementing line pumping and fluorescence with a low-resolution input spectrum can over-produce line strengths by as much as a factor of 2 . This is because model stellar absorption features become shallower with decreasing resolution, effectively increasing the number of photons in a wavelength bin that are able to pump Hydrogen atoms in 
the nebula (If, as might be possible for studies of the ISM surrounding emissionline stars, an emission spectrum is used, then decreased resolution would provide fewer photons for line pumping). Spectral resolutions that can be relied upon to give a realistic contribution from pumping and fluorescence are $\lambda / \Delta \lambda>2 \times 10^{3}$ in the ultraviolet (UV) and optical for the CLOUDY code. As an example of the kinds of spectra that do not meet this requirement, Starburst99 [120], the Castelli \& Kurucz plane-parallel stellar atmosphere models [25], and the TLUSTY suite of stellar atmostphere spectra, including those made specifically for CLOUDY [83]. All of these models were tested and found to produce errors of around the same magnitude, though only the Starburst99 spectrum is used below (Section 5.5) as an example of the pit-falls of low-resolution input spectra.

Using the high-resolution spectrum from model M2 in Luridiana et al. [124] as an input, I ran separate CLOUDY models that took into account pumping/fluorescence or else assumed Case B recombination theory only for two different model geometries: (1) A constant density model; and (2) a constant pressure model.

The constant density model is often utilized in the literature; e.g. the Case B calculations of Hummer \& Storey [85] and Storey \& Hummer [197] cover a wide range of possible densities to account for collisional rates, and more recent models from studies of BCDs [96] to $\mathrm{H}$ II regions [232] make use of constant densities as fiducials when more information about density is unknown. 
Where constant density models can offer general insight, models with density gradients often mimic real $\mathrm{H}$ II region morphologies. The cause of a density gradient in N49 was studied in depth in Draine [48] and found to be caused by a combination of radiation pressure and stellar winds. So-called "blister" H II region geometries, similar to that found at the center of the Orion Nebula, model density gradients assuming either constant pressure inside the region (gas and radiation pressure only in most cases) or else stellar winds pushing a substantial portion of the ionized gas into the ISM [60]. Yeh et al. [232] utilize such constant pressure models for studies of nebular diagnostics.

To quantitatively contrast the effects of geometry on line strengths, as well as the contribution of pumping/fluorescence on observed Hydrogen lines, I have run models with both constant density and constant pressure (static). For the constant density models, a gas density of $100 \mathrm{~cm}^{-3}$ was used, and a neutral column of $10^{21} \mathrm{~cm}^{-3}$ was placed along the line of sight, which is a realistic number for giant $\mathrm{H}$ II regions [23]. The ionizing flux at the inner edge of the cloud was set to $\mathrm{Q}\left(\mathrm{H}^{0}\right)=1.2 \times 10^{52}$ photons per second. The $\mathrm{H}$ II region is dusty. In addition, the high-resolution M2 spectrum from Luridiana et al. [124] was used so that the effect of pumping/fluorescence was not exaggerated. The results are shown in Table 5.4. For the $\mathrm{H} \alpha$ line, pumping/fluorescence contribute about 8-9\% of the emergent flux; for the $\mathrm{H} \beta$ line the contribution is $13 \%$; and for the ratio, $\frac{H \alpha}{H \beta}$, which is often used as an extinction tracer, the contribution from pumping/fluorescence is only $\sim 4 \%$. 


\begin{tabular}{|c|c|c|c|c|c|c|}
\hline & \multicolumn{6}{|c|}{$\mathrm{n}_{\text {gas }}=10^{2} \mathrm{~cm}^{-3}$} \\
\hline \multirow[t]{2}{*}{ Line } & \multicolumn{2}{|c|}{ Full H Model } & \multicolumn{2}{|c|}{ Case B } & \multicolumn{2}{|c|}{$\%$ difference } \\
\hline & intrinsic & emergent & intrinsic & emergent & intrinsic & emergent \\
\hline $\mathrm{H} \alpha$ & 0.161 & 0.0565 & 0.148 & 0.0521 & 8.8 & 8.4 \\
\hline $\mathrm{H} \beta$ & 0.0579 & 0.0156 & 0.0512 & 0.0138 & 13 & 13 \\
\hline$\frac{H \alpha}{H \beta}$ & 2.78 & 3.62 & 2.89 & 3.78 & 3.8 & 4.2 \\
\hline
\end{tabular}

The constant pressure model is a spherical nebula with $R_{\text {in }}$ of $1 \mathrm{pc}$, gas density at $\mathrm{R}_{\text {in }}$ of $1 \mathrm{~cm}^{-3}$, and an outer radius defined by when the ionization fraction reaches 0.05. The ionizing flux at the inner edge of the cloud was set to $\mathrm{Q}\left(\mathrm{H}^{0}\right)=1.2 \times 10^{52}$ photons per second. The $\mathrm{H}$ II region is dusty. The volume-average density of this model is $10^{4} \mathrm{~cm}^{-3}$, in keeping with similar blister $\mathrm{H}$ II regions [60]. The results of this model, shown in Table 5.4, are intriguing: the contribution of pumping/fluorescence to the emergent $\mathrm{H} \alpha$ and $\mathrm{H} \beta$ fluxes are $0 \%$ and $1.8 \%$, and the contribution to the $\frac{H \alpha}{H \beta}$ ratio is $1.8 \%$. The reason for the lower pumping/fluorescence contribution in the constant pressure model is because the collisional de-excitation rates are higher in the higher volume-averaged density gas. 


\begin{tabular}{|c|c|c|c|c|c|c|}
\hline \multirow{3}{*}{ Line } & \multicolumn{6}{|c|}{$\mathrm{P}_{\text {gas }}+\mathrm{P}_{\text {rad }}=$ constant } \\
\hline & \multicolumn{2}{|c|}{ Full H Model } & \multicolumn{2}{|c|}{ Case B } & \multicolumn{2}{|c|}{$\%$ difference } \\
\hline & intrinsic & emergent & intrinsic & emergent & intrinsic & emergent \\
\hline $\mathrm{H} \alpha$ & 134 & 57.5 & 137 & 57.5 & 2.2 & 0.0 \\
\hline $\mathrm{H} \beta$ & 48.0 & 17.4 & 48.3 & 17.1 & 0.6 & 1.8 \\
\hline$\frac{H \alpha}{H \beta}$ & 2.79 & 3.30 & 2.84 & 3.36 & 1.8 & 1.8 \\
\hline
\end{tabular}

For low-density, constant density models of H II regions embedded in neutral material, the contribution of pumping and fluorescence to the low-order Balmer line fluxes is on the order of $8-15 \%$ - a significant fraction. Since pumping contributes to both lines in a similar fashion, the contirbution of pumping/fluorescence on line ratios is significantly lower, $\sim 4 \%$. For constant-pressure H II region models, higher average densities increase the contribution of collision de-excitation to the line flux so that pumping/fluorescence only account for $>2 \%$ of the line flux.

My first conclusion from running these models is that blister $\mathrm{H}$ II region models, those whose density gradients can be easily modeled with constant pressure models, show that the contribution to the line fluxes and ratios of pumping/fluorescence is negligible. Case B should be used when only low-resolution input spectra and/or spectral shape is not known with great accuracy in a blister H II region model so that the issues with spectral resolution described in Luridiana et al. [124] are not repeated. 
My second conclusion from running these models is that, when constant density models are invoked, the contribution of pumping and fluorescence to the Hydrogen line fluxes is non-negligible. This is not explicitly stated in constant-density treatments using CLOUDY in the modern literature (e.g. Izotov \& Thuan [96], Yeh et al. [232], Verdolini et al. [214]) and is therefore cause of some concern. In the case of constant-density models, if Case B is assumed, the missing contribution from pumping/fluorescence will be filled by enhanced ionizing luminosities or perhaps larger filling factors, though this effect will be at the less than $15 \%$ level. If, however, the full Hydrogen model in CLOUDY is used, then users must utilize high-resolution spectra in order to mitigate the pumping/fluorescence contribution to the line fluxes. In the cases of Yeh et al. [232] and Verdolini et al. [214], they use Starburst99 spectra. Because using these low-resolution input spectra will likely over-produce the pumping/fluorescence contribution to the line by as much as a factor of 2 as stated in Luridiana et al. [124], such an error will lead to serious errors in data interpretation. We will now discuss the magnitude of this problem.

\subsection{The Use of Low-Resolution or Blackbody Spec- tra with CLOUDY}

Given the use of low-resolution Starburst99 spectra in otherwise sophisticated treatments of $\mathrm{H}$ II regions in, for instance, Yeh et al. [232] and Verdolini et al. [214], I wish to determine the magnitude of the problem when low-resolution 
input spectra are used, and to further determine the best solution when only low-resolution input spectra are available.

To that end, grids of constant-density $\left(100 \mathrm{~cm}^{-3}\right)$ models varying total column density and ionization parameter are made. To determine appropriate ranges for the ionization parameter, $U=\frac{Q\left(H^{0}\right)}{4 \pi R_{i n}^{2} c n}$, and the total column density, $N_{H}$, a number of $\mathrm{H}$ II regions parameters, from those surrounding single stars to those surrounding super star clusters, was organized as shown in Table 3. Considering the values found, values for $U$ of $0.1-10^{4}$ should cover most observable cases. Similarly, for total column, values in the range $N_{H} \sim 10^{19}-10^{23}$ are reasonable. However, since very high column densities require the introduction of molecular material and high extinctions (see Table 4), the grids calculated here stop at $10^{23.5} \mathrm{~cm}^{-2}$. 
Table 3. $U$ and $N_{H}$ Values for Regions of Interest

\begin{tabular}{|c|c|c|}
\hline Region Type & $U$ & $N_{H}\left(\mathrm{~cm}^{-2}\right)$ \\
\hline single stars \& & $\sim 0.1-10^{3} a$ & $\sim 10^{19-20}$ \\
\hline \multicolumn{3}{|l|}{ small OB assocns } \\
\hline Galactic HıI regions & $\sim 0.1-10^{b}$ & $\sim 10^{19-22}$ \\
\hline SSCs & $\sim 1-10^{3 c}$ & $\sim 10^{21-22}$ \\
\hline \multirow[t]{2}{*}{ UCHIIs } & $<0.1^{d}$ & $\sim 3 \times 10^{21}($ ionized $)$ \\
\hline & & $\gtrsim 5 \times 10^{23}$ (molecular cloud $)$ \\
\hline embedded SSCs & $\sim 0.1-1^{e}$ & $\gtrsim 10^{23}$ \\
\hline Orion Nebula & $\sim 0.3^{b}$ & $\sim 10^{21-22}$ \\
\hline \multicolumn{3}{|l|}{ (Galactic HII region) } \\
\hline NGC 604 & $\sim 10$ & $\gtrsim 10^{22}$ \\
\hline \multicolumn{3}{|l|}{ (superassociation) } \\
\hline 30 Doradus & $\sim 3^{g}$ & $\gtrsim 10^{22}$ \\
\hline (SSC in the LMC) & & \\
\hline
\end{tabular}

${ }^{a}$ The values for stars and OB associations were calculated using ionizing fluxes from Osterbrock \& Ferland [147] and assuming an inner cloud radius of 1 pc.

${ }^{b}$ Average values from Osterbrock \& Ferland [147]

${ }^{c}$ Calculated using values from Whelan et al. [218]

${ }^{d}$ From Wood \& Churchwell [224]

${ }^{e}$ Calculated using values from Johnson et al. [100]

${ }^{f}$ Pellegrini et al. [153] 
Table 4. Parameter Conversions

\begin{tabular}{lll}
$N_{H}$ & $A_{V}$ & $\mathrm{M}_{\text {cloud }}\left(\mathrm{M}_{\odot}\right)$ \\
\hline $10^{19}$ & $5.5 \times 10^{-3}$ & $1.01 \times 10^{2}$ \\
$10^{20}$ & $5.5 \times 10^{-2}$ & $1.04 \times 10^{3}$ \\
$10^{21}$ & $5.5 \times 10^{-1}$ & $1.36 \times 10^{4}$ \\
$10^{22}$ & 5.5 & $7.77 \times 10^{5}$ \\
$2 \times 10^{22}$ & 9.9 & $4.31 \times 10^{6}$ \\
$3 \times 10^{22}$ & 17 & $1.27 \times 10^{7}$ \\
\hline
\end{tabular}

The input spectrum is a 3 Myr old starburst spectrum with a Kroupa initial mass function [110] at solar metallicity with Geneva high-mass loss stellar atmospheres, as recommended in the Starburst99 code [120]. The 3 Myr old spectrum was chosen because its ultraviolet continuum is consistent with young stellar populations and is therefore appropriate for $\mathrm{H}$ II region studies. The model spectrum is shown along with other spectra in the 1-10 Myr time span for comparison in Figure 5.1.

I plot the ionization fraction across the models grid for the $3 \mathrm{Myr}$ old grid in Figure 5.2. For grid numbers in the region where some neutral material exists, we expect that multiple scattering events in the neutral material will allow the line fluorescence results to converge with the Case B results. However, in order to fully study the effect of neutral columns on observed quantities, we would need to run new grids that properly accounted for radiation pressure balance between the HII region and neutral surroundings. As a result, model values at high column density $\left(>10^{21} \mathrm{~cm}^{-2}\right.$ and low ionization parameter $(<1)$ should be treated with caution. 


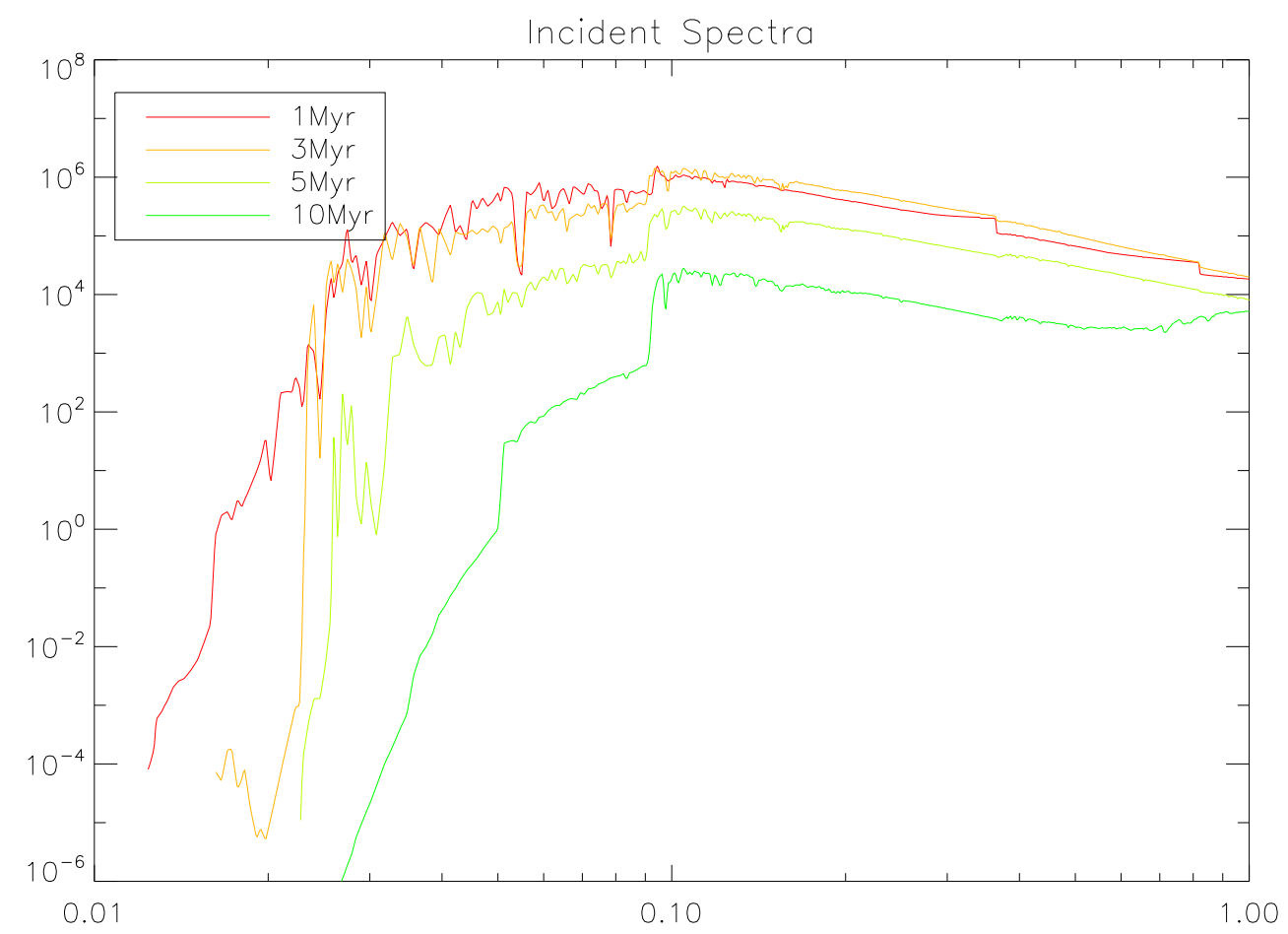

Figure 5.1: The 1-10 Myr old Starburst99 SEDs for a Kroupa IMF and upper mass cutoff of $120 \mathrm{M}_{\odot}$.

The ratio of the emergent $\mathrm{H} \beta$ flux from the pumping/fluorescence and Case $\mathrm{B}$ model grids is shown in Figure 5.3. This line is often used as a benchmark for spectroscopic flux calibration, against which all other line fluxes are compared [e.g. $22,96]$. It is also used as a measure of the ionizing flux when Ly $\alpha$ fluxes are not available [120], and is therefore an important line to model.

The effect of continuum pumping and fluorescence is substantial when using the low-resolution Starburst99 spectrum. Figure 5.3 shows that the ratio of the pumping/fluorescence fluxes and the Case B fluxes across the tested parameter space are as has as a factor of 2 in the ionized region (as first shown in Luridiana et al. [124]), and still as high as 1.8 in the neutral material (cf. Figure 5.2). Compared 


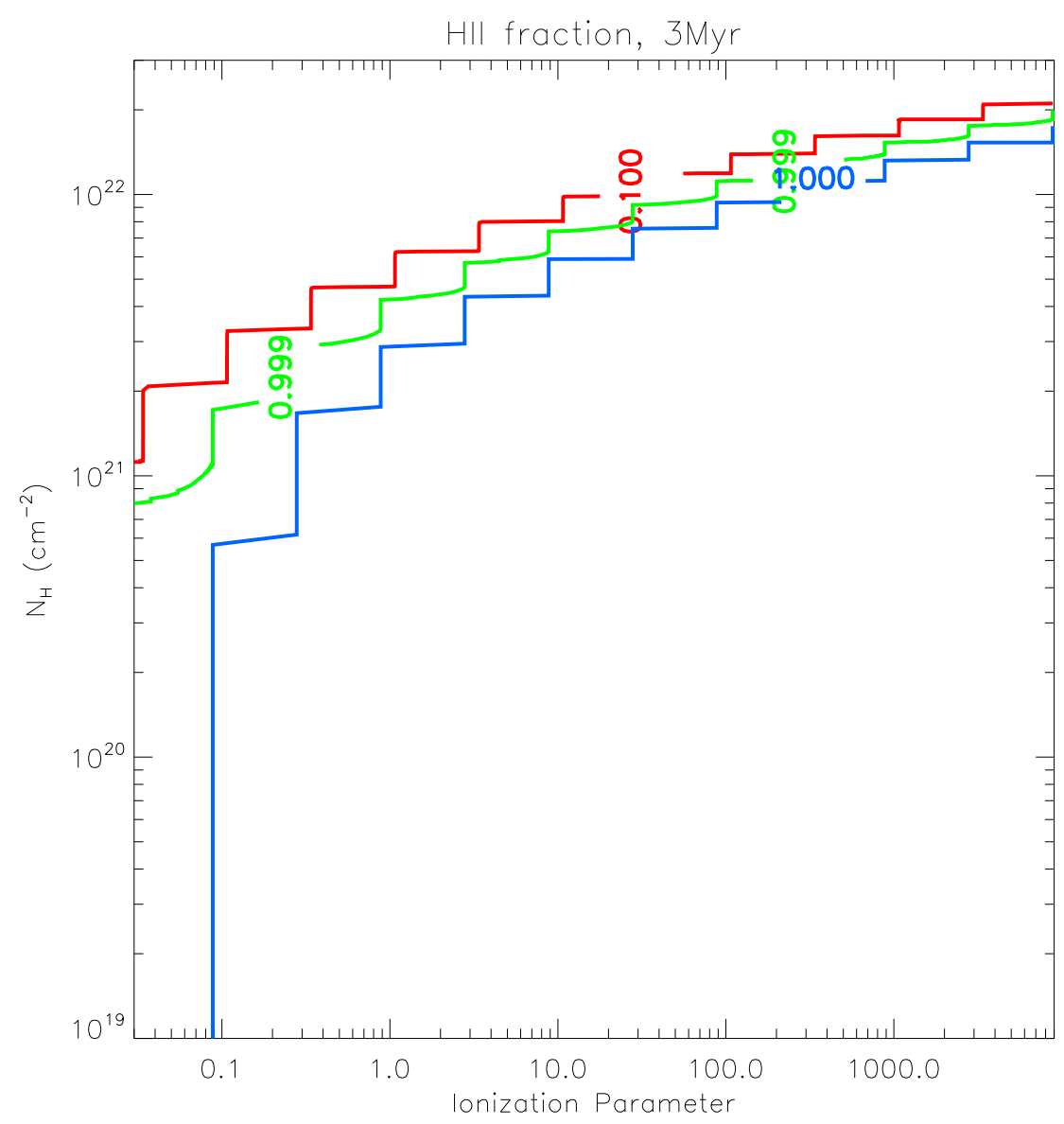

Figure 5.2: The fraction of gas that is ionized across the model grid using a 3 Myr old starburst SED for the input ionizing spectrum. I urge caution when interpreting results that include neutral material, since I have made no attempt at pressure balance in these models.

to the mere $13 \%$ difference demonstrated in Section 5.4 when a high-resolution input spectrum was used to interpret the $\mathrm{H} \beta$ line, shows that the low-resolution spectrum has had a significant impact on the modeled line flux, increasing the emergent $\mathrm{H} \beta$ line flux by more than $50 \%$ to what it should have been.

The ratio of the emergent $\mathrm{H} \alpha / \mathrm{H} \beta$ line fluxes are often used as an extinction measure [e.g. 23, 96]. By comparing the observed value with a canonical value, typically 2.86 for a $10^{4} \mathrm{~K}$ gas at low density, an $\mathrm{E}(\mathrm{B}-\mathrm{V})$ or $A_{V}$ value is determined for 


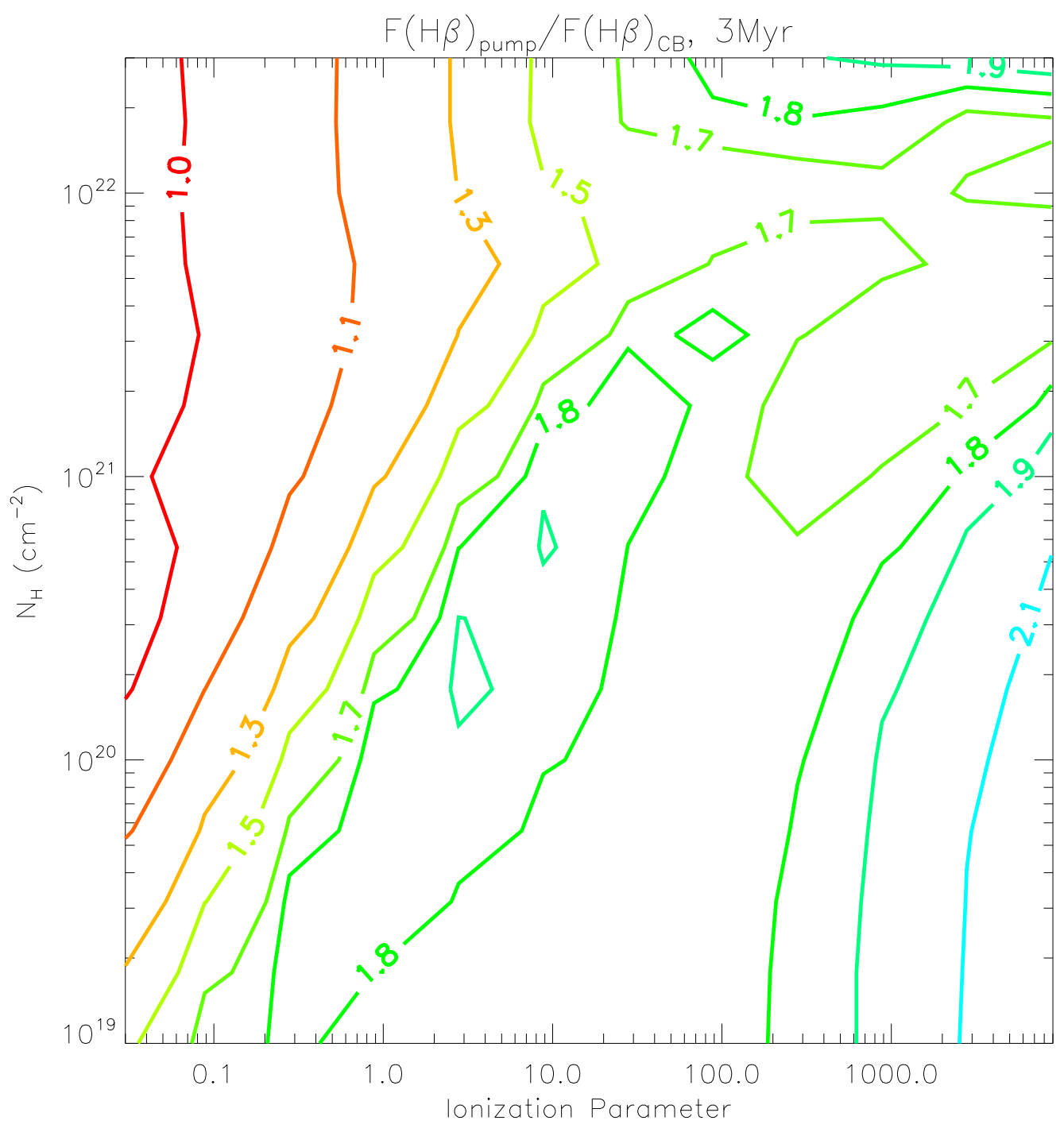

Figure 5.3: The ratio of the emergent $\mathrm{H} \beta$ flux for the pumping/fluorescence and Case B model grids as a function of $U$ and $N_{H}$ using the 3 Myr starburst SED.

the assumed metallicity, and from there an assumed de-reddening curve (either derived from a known dust size distribution or else assumed) is used to correct a spectrum's flux. Figure 5.4 shows the ratio of the $\mathrm{H} \alpha / \mathrm{H} \beta$ ratios for the pumping/fluorescence and Case B model grids in the $N_{H}-U$ parameter space. The two models, pumping/fluorescence and Case B, give overall close results, but are 


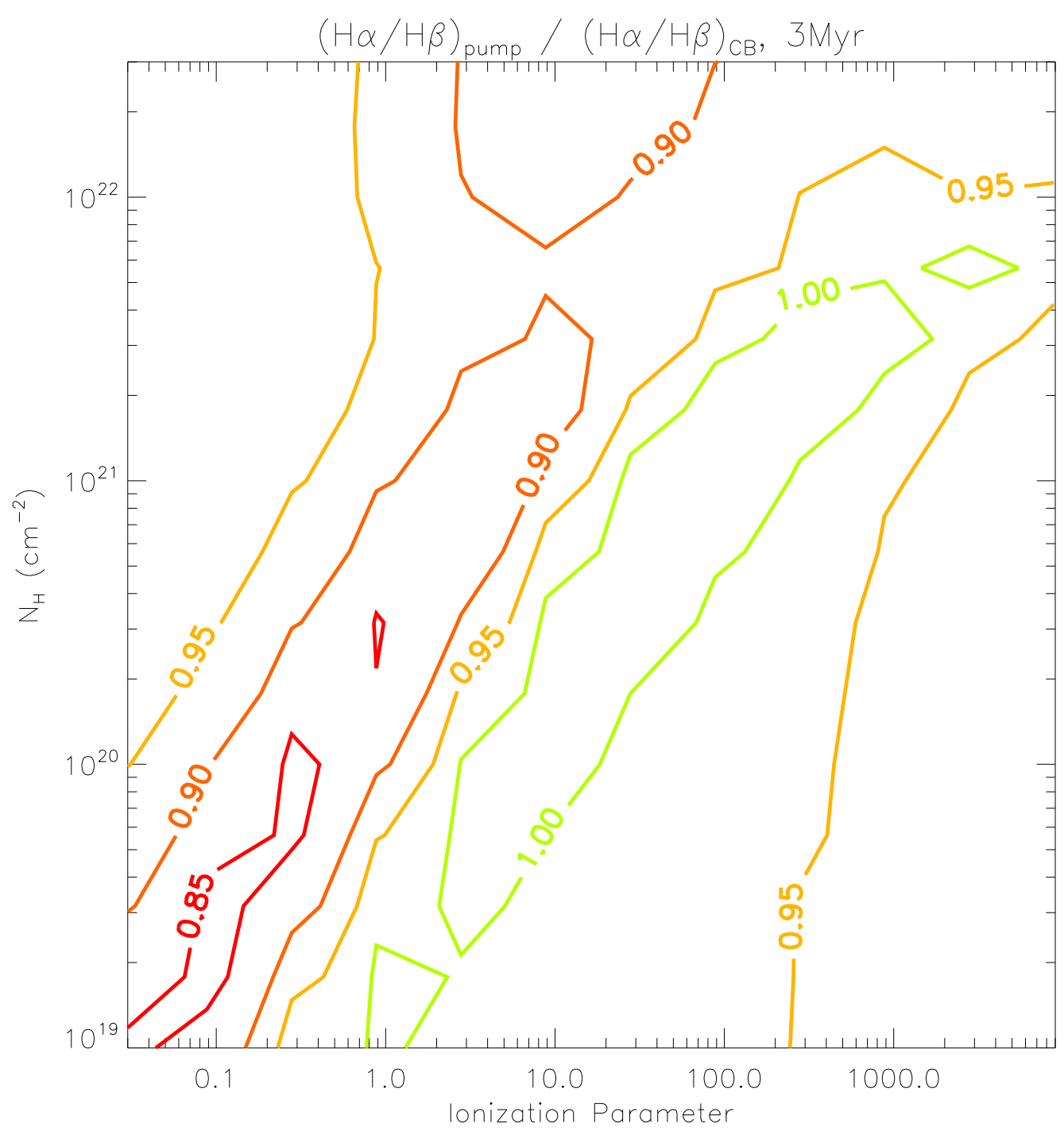

Figure 5.4: The ratio of the emergent $\mathrm{H} \alpha / \mathrm{H} \beta$ flux ratios for pumping/fluorescence and Case $\mathrm{B}$ model grids, using the $3 \mathrm{Myr}$ old starburst SED in $U-N_{H}$ parameter space.

still about five times more different than those using high-resolution input spectra as shown in Section 5.4. The difference can be substantial: for a constant density model with a neutral column of $10^{21} \mathrm{~cm}^{-2}$, the contribution to the emergent $\mathrm{H} \alpha / \mathrm{H} \beta$ ratio from pumping/fluorescence is only $4.2 \%$, whereas for the same parameter in this space $\left(U=34, N_{H}=10^{22} \mathrm{~cm}^{-2}\right)$, the contribution is $10 \%$. 
In conclusion, this simple test across the wide swathe of reasonable parameter space shows that the effect of over-produced pumping/fluorescence contribution to the line fluxes and ratios is not limited to certain cases. In all perceivable cases of interest in $\mathrm{H}$ II region studies, low-resolution input spectra have been shown to greatly enhance the contribution of pumping and fluorescence to the Hydrogen line fluxes and ratios.

\subsection{Conclusions}

CLOUDY [62] is a highly sophisticated code that, among other things, contains a full Hydrogen atom including all known radiative effects, notably among them line pumping from the continuum and subsequent line fluorescence - the processes neglected in Case B recombination theory. As a result, CLOUDY offers an appealing tool with which to build models of $\mathrm{H}$ II regions. Using CLOUDY, I have modeled common geometries invoked in the literature (constant density and static constant pressure) in an attempt to determine when the full Hydrogen atom and all of its radiative processes should be used in stead of simple Case B recombination theory. I first ran models using a high wavelength-resolution starburst spectrum as the ionizing source from Luridiana et al. [124], and found that for the constant density models, pumping/fluorescence contributed $8 \%$ to the emergent $\mathrm{H} \alpha$ line flux, $8 \%$ to the $\mathrm{H} \beta$ line, and only $4.2 \%$ to the $\mathrm{H} \alpha / \mathrm{H} \beta$ line ratio. By contrast, in the constant pressure model, pumping/fluorescence contributed $0 \%$ to the emergent 
$\mathrm{H} \alpha$ line flux, $1.8 \%$ to the $\mathrm{H} \beta$ line, and $1.8 \%$ to the $\mathrm{H} \alpha / \mathrm{H} \beta$ line ratio. It is quite clear, then, that when high-resolution input spectra are available for use, Case B is more than sufficient when considering constant-pressure $\mathrm{H}$ II region models and is also suitable in constant density models if only line ratios are being modeled. If modeling line fluxes accurately with constant density models, then a treatment using the full Hydrogen atom, not just Case B, is necessary.

However, many treatments in the modern literature make use of constant density models with either blackbody spectra as inputs or low-resolution spectra such as are available from Starburst99, Castelli \& Kurucz, and TLUSTY [e.g. 96, 214, 232]. In these cases, my grids in ionization parameter and total column depth show that such a treatment is likely to vastly exaggerate the contribution by pumping/fluorescence to Hydrogen line fluxes and ratios. As an example using a Starburst99 spectrum for the source, the $\mathrm{H} \beta$ flux could be enhanced by $70-90 \%$ attributed to pumping/fluorescence, simply because the low-resolution spectrum artificially raises the photospheric line depths and thereby increases the number of virtual photons available to pump Hydrogen atoms. The effect is much less dramatic for the $\mathrm{H} \alpha / \mathrm{H} \beta$ line ratio, being no more than 15\%; however, even this is 3 times more than what was observed using a high-resolution input spectrum.

It is up to the modeler to determine the optimal configuration for the system they are modeling. However, I offer these simple guidelines to prevent mistakes caused by invoking incorrect $\mathrm{H}$ II region geometry or else by using a too coarsely resolved input spectrum. 
(1) for resolved systems, a constant pressure model will be closer to the observed density profile than a constant density model [e.g. 48]. In this instance, Case B will be more than adequate to model both line fluxes and line ratios accurately.

(2) In the case of distant extragalactic systems where constant density models are invoked, then the best and most accurate treatment calls for including pumping/fluorescence so long as a high-resolution source spectrum is available for use.

(3) In the absence of a high-resolution input spectrum, when either blackbodies or low-resolution spectra are used instead, then Case B should once again be assumed, with only minor errors expected from the missing pumping/fluorescence contribution. Invoking pumping/fluorescence with low-resolution spectra or blackbodies will grossly overestimate the line fluxes, seriously impacting data interpretation. 


\section{Chapter 6}

\section{Conclusions and Future}

\section{Directions}

\subsection{Conclusions}

The overarching question that this thesis addresses is: what are the properties of massive star clusters from their deeply embedded beginnings through their HII region phase? Since high-resolution infrared and submillimeter data is only going to become available to address this question in the coming decade and beyond, much of what I have presented is theoretical, only relying on data to study the HII region and PDR associated with a relatively nearby superassociation in the SMC, as an example of this stage of evolution. 
Chapter 1 provides a background of observations and theoretical predictions. It discusses initial observations dating back to the early 1970s, early conceptual errors regarding the nature of super star clusters, and the data, both optical and radio, that both fixed these errors and provided our first insights about the nature of massive star forming regions. Using energy and momentum conservation, I provide a more-than-basic analytical introduction to studying massive star cluster formation, including a simple way to account for pressure due to either turbulence or magnetic fields. It is interesting that, in this analysis, the addition of turbulence or magnetic fields of up to $50 \%$ of the extant energy budget in the system does not meaningfully change the minimum amount of gas that must be converted into stars for the system to remain bound; whereas an analysis considering gravity alone requires a $50 \%$ conversion rate, $\pm 50 \%$ magnetic field or turbulent energy only changes this conversion rate from 40-70\%. I recognize, however, that this analysis is of limited use, unless the entire system mass is allowed to change; recent data and simulations suggest that the formation cloud can increase in mass by roughly a factor of 2 over the course of the star cluster's formation and early evolution, making the meaning of the term "star formation efficiency" rather difficult to grasp outside of theoretical prescriptions. In addition, I review the current knowledge of cloud dispersal and HiI region expansion, and how these principles likely translate for massive clusters.

Chapters 2-5 of this thesis provide meaningful and useful numerical predictions and observations of massive star cluster environments from their deeply embedded 
phase through the HiI region phase. I will briefly summarize the main points below.

In Chapter 2, I present dust radiative transfer models of embedded super star cluster geometries. By including PAH/VSG templates, I incorporate a significant emission component to the mid-infrared spectrum that make these models an excellent comparison sample to future observations with the James Webb Space Telescope (JWST). In three dimensions, we implemented a fractal dust geometry that mimics what is seen in the interstellar medium. This geometry is able to reproduce recent observations of "light leakage" from embedded super star cluster geometries. However, this clumpiness means that the infrared spectral energy distributions are degenerate, providing not only hundreds of differently-shaped spectra per model depending on sightline, but also similar spectra for different models. In order to break this degeneracy, a method derived from principal component analysis was employed that was able to determine the best colors for tracing the various input parameters into the models. We determined what colors are most likely able to trace the percentage of clumpy material in the envelope, what the inner and outer radii are, and what the dust mass is. These models were compared to photometry of ultra-compact HiI regions, Milky Way low-mass analogues of embedded super star clusters, to resounding success, and look forward to these models being useful when $J W S T$ is available to the astronomical community.

Chapter 3 extends the modeling efforts presented in Chapter 2 to molecular line emission, with the intention of providing a similar body of models for comparison to future Atacama Large Millimeter/sunmillimeter Array (ALMA) observations. 
Due to the restricted availability of three-dimensional codes at the time this project was undertaken, I restricted the models to one dimension. Our most significant result was that the model geometries, except clumping, from Whelan et al. [218] are hugely superluminal in $\mathrm{CO}$ and $\mathrm{HCN}$ models of their envelopes, even after detailed photodissociative chemical models are used to solve for the abundances in the cloud. Considering that embedded super star clusters are likely formed out of collapsing giant molecular clouds, I instigated a density gradient in the model envelopes and re-ran the simulations. I was able to model the predicted $\mathrm{CO}$ and HCN line luminosities and place them near correlations with other data; however, the line luminosities were not able to distinguish between different power law density values. Without a detailed knowledge of the geometry of a collapsing giant molecular cloud $\left(10^{7} \mathrm{M}_{\odot}\right)$ and the effect that turbulence has on the line widths, it was impossible to provide useful and realistic 1-D models to the community. However, new advances in 3-D PDR and molecular line radiative transfer may mean that the tools are in place to begin to finally answer the questions necessary to run more detailed models.

Chapter 4 presents previously unpublished Spitzer Space Telescope/Infrared Spectrograph data of the giant HII region N66 surrounding the superassociation NGC 346 in the Small Magellanic Cloud. Using a novel reduction technique, we were able to separate out the extended emission in the slit from that of the unresolved point sources. Among the point sources were a spectrum of a massive embedded young stellar object, two stellar clusters exhibiting rarely seen silicate emission 
features, and what looks to be a Herbig AeBe star. Additionally, the analysis of the PAH bands revealed an anomalous shift in the $11.3 \mu \mathrm{m}$ PAH complex, a unique non-correlation between the 6.2 and $7.7 \mu \mathrm{m}$ bands, and a strong suggestion that processing of the PAH bands is taking place in the hard radiation field across the region. The $14 \mu \mathrm{m}$ emission seems to suggest that it originates primarily from very small grains because there was a linear correlation with radiation field strength and hardness; any nonlinearity would have suggested the presence of temperaturesensitive large grains. And lastly, we determined that most of the emission across the region arises from the diffuse medium, not the many infrared point sources. Studies of unresolved giant HiI regions in other low-metallicity systems should expect their infrared spectra to be dominated by the diffuse emission.

Chapter 5 is a detailed analysis of the effects of pumping and fluorescence in models of $\mathrm{H}$ II regions using the photoionization code, CLOUDY. In the case of constant-density models, using appropriately high wavlength-resolution input spectra yield realistic contributions of pumping and fluorescence to the Hydrogen lines, producing an extra $\sim 13 \%$ line flux in $\mathrm{H} \beta$ and $<4 \%$ in $\mathrm{H} \alpha / \mathrm{H} \beta /$ line ratio. When using input spectra which has low wavelength resolution, pumping and fluorescence contributions to the line fluxes are significantly over-produced (an additional 70-90\% $\mathrm{H} \beta$ line flux and $\sim 10-15 \%$ in $\mathrm{H} \alpha / \mathrm{H} \beta$ ratio). "Blister" $\mathrm{H}$ II region model geometries do not suffer nearly as much from pumping and fluorescence ( $<2 \%$ in $\mathrm{H} \beta$ and $\mathrm{Halpha} / \mathrm{H} \beta$ ratio). I implement grids of models to illustrate the magnitude of this effect in a wide variety of parameter space. These 
models illustrate the dangers of using simplified geometries and low-resolution ionizing spectra with modern codes, and suggest that, in spite of the sophistication of many codes, assuming Case B recombination theory and, when resolved data is available a blister $\mathrm{H}$ II region geometry, is in most cases the most robust solution.

\subsection{Future Directions}

The diverse body of work summarized above will require a large and evermore far-reaching set of solutions in order to answer the questions that remain. I wish to provide road plans for future works in all of the discussed areas.

(1) Dust and gas radiative transfer: One proposed idea for providing additional constraints to the degeneracies found in Chapter 2 is to use a full gas and dust radiative transfer code. Such a code will provide infrared atomic and ionic emission lines, whose relative strengths with sightline and geometry can be used as tracers of the ionized and neutral gas content in embedded super star clusters. While early tests with a certain code had to be abandoned because of programming issues, RADMC_3D offers a new opportunity with which to attempt this complete new set of models.

(2) Hydrodynamic simulations: Quantifying timescales for collapse and cluster formation, the effects of radiation on cloud size and structure (including turbulence and corresponding fractal structure), and possible variability are some of the things 
that hydrodynamics will be able to answer for super star clusters. Some of these questions should be answered before realistic molecular line emission models of embedded SSCs can be produced, so that the velocity structure can be properly mapped into the line profiles.

(3) 6.2 and $7.7 \mu \mathrm{m}$ PAHs: It was determined that the lack of correlation between the $6.2 \mu \mathrm{m}$ and $7.7 \mu \mathrm{m}$ PAH bands observed in N66 was not due to the reduction technique. However, is it systematic at low-metallicity? Or in relatively hard stellar radiation fields? In short, what is the physical interpretation of this curious result?

(4) Recombination lines in a variety of objects: Modeling nebular diagnostics for a variety of Galactic and extragalactic HiI regions will provide systematic information on the true error of assuming Case B for spectroscopic observations. Since optical spectroscopy has been used frequently to measure line emission from a large variety of astrophysical nebulae, there is a very large literature available from which data can be pulled, and corresponding objects for which the important parameters can be constrained, both assuming Case B and Cases C/D. 


\section{Appendix A}

\section{The Lucy Method of Computing}

\section{Radiative Equilibria with Monte}

\section{Carlo Techniques}

\section{A.1 INTRODUCTION}

The 'Lucy Method' is a method for computing radiative equilibria in Monte Carlo codes. It is presented in Lucy [123]. This is the method of radiative transfer through dust used in embedded star cluster environments [218] as coded by Barbara Whitney. Here is a short introduction, meant to act as a reference guide and an overview of the code. 


\section{A.2 The Lucy Method}

\section{A.2.1 Overview}

The Lucy Method is meant for Monte Carlo radiative transfer codes, i.e. those which rely on random sampling of a cumulative distribution function of the dust. The calculation uses quanta, which we refer to as photon packets, on account of the fact that each has a discrete energy. Thus they are not photons, since each packet has many, many times the energy of a single photon, but instead they act like a whole group of equal-energy photons working in unison (energy $\epsilon(\nu)=n h \nu$; how $\mathrm{n}$ is determined will be explained below).

To the discerning eye, the Lucy Method will look like the ill-fated Lambda Iteration technique, but there is one major difference that separates the two methods. Whereas Lambda Iteration is known to diverge in certain cases because the luminosity of the system is not fixed, the Lucy Method constrains the flux not to diverge between photon packet interactions. Technically, this means that an energy packet that is absorbed is re-emitted with a new frequency with the total energy of the packet held constant: $\epsilon\left(\nu_{e}\right)=\epsilon\left(\nu_{a}\right)$. With the flux set as a constant, the Monte Carlo radiation field can be solved as a solution to the equations of radiative transfer, as here described. 


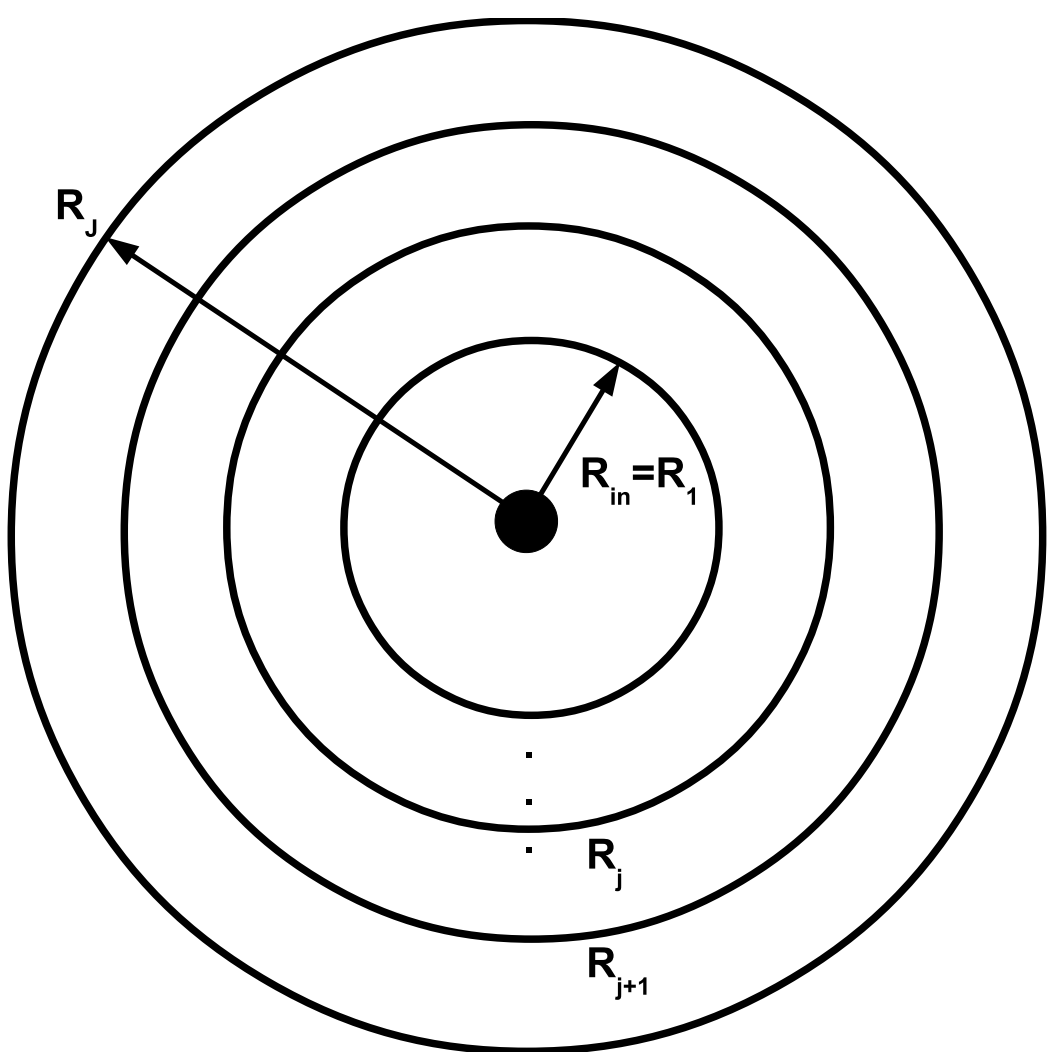

Figure A.1: This is an illustration of how the dusty envelope surrounding the central star cluster is discretized into a number of shells with inner radii $\mathrm{j}$ and outer radii $\mathrm{j}+1$.

\section{A.2.2 Initialization}

The code used in Whelan et al. [218] [based on the original 2-D code in 220], while capable of dealing with arbitrary dust geometries surrounding the photon source, is set to remain spherically symmetric for the time being. The dusty envelope is divided into $\mathrm{J}$ spherical shells; the $j^{\text {th }}$ shell has inner and outer radii of $R_{j}$ and $R_{j+1}$. This geometry is shown in Figure A.1. In the absence of fractally distributed dust in the envelope, the mass density, temperature, and scattering and absorption coefficients are held constant (known by their respective variable names $\rho, T, \sigma$, and $\left.k_{\nu}\right)$. 
The outward-directed radiation field is determined from the input spectrum of the central source: $I_{\nu}^{+}$. Then the mid-probability points $\nu_{k}$ of $K$ equal probability bins are calculated

$$
\frac{k-\frac{1}{2}}{K}=\frac{\int_{0}^{\nu_{k}} I_{\nu} d_{\nu}}{\int_{0}^{\infty} I_{\nu} d_{\nu}}
$$

where $k=1,2,3, \ldots . K$. For each randomly-selected value of $k$, the emitted photon packet is set to $\nu_{k}$. The initial cosine of the direction is set $\mu=\operatorname{sqrt}(z)$ where $\mathrm{z}$ is a random number in the interval $(0,1)$.

\section{A.2.3 Trajectories}

With the photon packet initialized and set free from the lower boundary of the dusty envelope, we compute its trajectory through the envelope.

In each shell of the envelope, the random flight path of any photon packet is set simply by the optical depth $\tau_{\nu}=-\ln (z)$, which sets the physical distance, $\ell$, that the photon travels to $\tau_{\nu}=\left(k_{\nu}+\sigma\right) \rho \ell$. If $\tau_{\nu}$ is low enough, the photon packet will simply leave the current shell and a new optical depth from the next shell will be computed. When the path length is shorter than the shell size, then the photon must either be scattered or absorbed by the dust in the shell. Scattering occurs if:

$$
z<\frac{\sigma}{k_{\nu}+\sigma}
$$


because then scattering is more likely than absorption. Otherwise, absorption occurs. Whether the photon packet is scattered or absorbed, it sets out in a new direction after the interaction. In the event of scattering, the frequency of the photon packet remains unchanged. However, the frequency is re-assigned in the event of absorption by:

$$
z=\frac{\int_{0}^{\nu} j_{\nu} d \nu}{\int_{0}^{\infty} j_{\nu} d \nu}
$$

where $j_{\nu}$ is the emissivity. For all interactions, the new cosine of the direction is selected by $\mu=-1+2 z$, which represents isotropic scattering or emission. N.b. that the re-assignment of $\nu$ using $\mathrm{z}$ is, in practice, replaced by a randomly selected value from equal probability emissivity bins. From the selected emissivity, the frequency is calculated. The two methods, indiscrete and discrete, are computationally identical.

\section{A.2.4 Monte Carlo Estimators}

In radiative equilibrium, the basic principle is that the rate at which matter absorbs energy from the radiation field should equal the rate at which matter emits energy.

The rate at which matter absorbs energy from the radiation field is:

$$
\dot{A}=4 \pi \int_{0}^{\infty} k_{\nu} J_{\nu} d \nu
$$


while the rate at which matter emits energy is:

$$
\dot{E}=4 \pi \int_{0}^{\infty} k_{\nu} I_{\nu} d \nu
$$

$J_{\nu}$ is given by the basic result that the energy density of the radiation field in the interval $(\nu, \nu+d \nu)$ is $4 \pi J_{\nu} d \nu / c$. The photon packet contributes energy $\epsilon(\nu)=\epsilon_{0}$ to the shell it is in. Take $\ell$ to again be the path length between successive events, including boundary crossings, then the packet's trajectory contributes an amount $\epsilon_{0} / \Delta t$ to the time-averaged energy content of the shell, where $\delta t=\ell / c$ and $\Delta t$ is the duration of the Monte Carlo experiment (i.e. how much time passes between when the photons first enter the envelope and the last photon leaves it). From this, we can determine the monochromatic mean intensity:

$$
J_{\nu} d \nu=\frac{1}{4 \pi} \frac{\epsilon_{0}}{\Delta t} \frac{1}{V} \sum_{d \nu} \ell
$$

for volume $V$. Correspondingly, the integrated mean intensity is:

$$
J=\frac{1}{4 \pi} \frac{\epsilon_{0}}{\Delta t} \frac{1}{V} \sum \ell
$$

The Monte Carlo estimator for the absorption rate is therefore:

$$
\dot{A}=\frac{\epsilon_{0}}{\Delta t} \frac{1}{V} \sum k_{\nu} \ell
$$


and this is the expression that is solved iteratively to find the radiative equilibrium solution.

\section{A.2.5 Temperature corrections}

The central star cluster's luminosity is fixed to some value, as well as the number of photon packets. These quantities, plus the total interaction time, are related to produce the amount of energy contained in one photon packet:

$$
L(\infty)=N_{\infty} \frac{\epsilon_{0}}{\Delta t}
$$

where $L(\infty)$ is the cluster's luminosity, which is the bolometric luminosity of the end result, and $N_{\infty}$ is the number of escaped photons. The $\dot{A}$ solved for using the $\epsilon_{0} / \Delta t$ will not balance with $\dot{E}$. To obtain a modified dust temperature, $\dot{E}$ for the shell volume is written as

$$
\dot{E}=4 \pi k_{P} B
$$

where $k_{P}$ is the Planck-mean absorption coefficient and $B=\frac{\sigma}{\pi} T^{4}$ is the Planck function. The temperature distribution for the next iteration is then given by

$$
B=\frac{\dot{A}}{4 \pi k_{P}}
$$


where $\dot{A}$ from the last iteration is used. This is the part of the process that looks so suspiciously like the lambda iteration method, where $B=\frac{k_{J}}{k_{P}} J$ is typically written instead of the above-given formula. But the difference is that $B=\frac{k_{J}}{k_{P}} J$ does not correct for the luminosity of the shell, whereas that information is carried in the Monte Carlo radiation field because it is used to calculate $\epsilon_{0}$.

Now the boundary temperature should be iteratively improved, first by integrating $I_{\nu}^{+}$to give $L^{+}$so that it equals the bolometric luminosity given previously:

$$
L^{+}=4 \pi R_{\text {inner }}^{2} \times \sigma T_{b}^{4}=N \frac{\epsilon_{0}}{\Delta t}
$$

where $T_{b}$ is the boundary temperature. So with the $\epsilon_{0} / \Delta t$ obtained as explained earlier, $L^{+}$is found and $T_{b}$ determined.

\section{A.3 Important Departures from the Lucy Method}

The radiative transfer code developed for use in super star cluster theory is a threedimensional code. In addition to the radius, the geometry must also be explained by an angle $\phi$ from the zenith and $\theta$ around the principal axis. Radiative transfer in specific shells as described in the Lucy Method above are therefore confined to small grid locations, each with specific radius, $\phi$, and $\theta$ coordinates. In Whelan et al. [218], grids with 200 radial, $10 \phi$ and $20 \theta$ positions were invoked. This 
was sufficient to study the effects of a clumpy, fractal dust distribution in the embedding envelope. The fractal equations are presented in the next appendix.

Due again to the three-dimensional nature of the study, the isotropic scattering assumption of the Lucy Method is untenable; in the case of one dimension, isotropic scattering sends half of the photon packets forward, and half of them backward. This is an unphysical assumption in dusty environments, where the dust strongly forward-scatters the light from a star or star cluster. We therefore use the HenyeyGreenstein function to scatter light off of dust particles in the dusty envelope [74]. The phase function adopted by Henyey \& Greenstein is:

$$
\Phi(a)=\frac{\gamma\left(1-g^{2}\right)}{4 \pi} \frac{1}{\left(1+g^{2}-2 g \cos (a)\right)^{\frac{3}{2}}}
$$

where $a$ is the phase angle defined as deviation from the forward direction, $\gamma$ is the albedo, and $g$ is a measure of the asymmetry of the phase function. For $g=0$, isotropic scattering is recovered, and $g=1$ is no scattering at all (i.e. all the light is scattered in the forward direction). The expression for $g$ is

$$
\gamma g=\int \Phi(a) \cos (a) d \omega=\frac{1}{2} \int_{0}^{\pi} \Phi(a) \cos (a) \sin (a) d a
$$

Henyey \& Greenstein [74] showed that for $\gamma=0, \frac{1}{3}, \frac{2}{3}$, and $1, g>1,=1,0.9$, and 0.8 respectively; for high or low albedo values, the dust strongly forward-scatters 
Appendix A. The Lucy Method of Computing Radiative Equilibria with Monte Carlo Techniques

the light. The data used were individual measurements of the diffuse radiation in the Taurus-Aurigae and Cygnus 'dark clouds.' 


\section{Appendix B}

\section{The Fractal Dust Distribution of}

\section{a Super Star Cluster Embedding}

\section{Envelope}

\section{B.1 Introduction}

Interstellar clouds appear to have fractal geometries [56, and references therein] and there is good evidence that the same is probably true of deeply embedded, young super star cluster embedding envelopes. This appendix outlines the fractal equations used to estimate that fractal shape, as given by Elmegreen [56]. 


\section{B.2 Fractal Dust Distributions}

There is an assumed mass-size relation for cloud size in the envelope, as well as distribution functions for cloud size and mass.

$$
M \propto S^{\kappa}
$$

$$
n(S) d S \propto S^{-(1+D)} d S
$$

$$
n(M) d M \propto M^{-1-\frac{\kappa}{D}} d M
$$

$D$ is the volume fractal dimension. It should be noted that these relations are useful over a wide range of sizes, from $0.01 \mathrm{pc}$ to over $100 \mathrm{pc}$, and also that star formation occurs on these same size scales.

Fractal dust distributions are made with $H$ hierarchical levels. Three-dimensional Cartesian coordinates are chosen so that for random numbers $r_{i} \in(0,1)$, the $\mathrm{x}$ coordinates at each level $H$ are given as follow, and the same holds for y and Z:

$$
x_{1}=\frac{2\left(r_{1}-0.5\right)}{L^{1}}+0.5
$$




$$
\begin{gathered}
x_{2}=x_{1}+\frac{2\left(r_{2}-0.5\right)}{L^{2}} \\
x_{3}=x_{2}+\frac{2\left(r_{3}-0.5\right)}{L^{3}} \\
x_{H}=x_{H-1}+\frac{2\left(r_{H}-0.5\right)}{L^{H}}
\end{gathered}
$$

where $L$ is used to subdivide the structure from one level to the next. At each level, $N$ new positions are substituted for each $(\mathrm{x}, \mathrm{y}, \mathrm{z})$ position in the previous level so that there are a total of $N^{H}$ positions after $H$ levels. A $L^{3 H}$ cubical grid is placed around all of the points.

Elmegreen \& Efremov [55] found that $D \approx 2.3$ is standard for clouds with $M \propto S^{D}$, and that this happens to apply to laboratory fluids as well. Using this as a starting point, several values of $L$ and $N$ can be used to produce this result. $D=\frac{\log N}{\log L}$ so $L=3$ and $N=12$, and $L=2$ and $N=5$ could either reproduce the data. choosing $H$ so that the number of final points is about the same ( $H=4$ and 6 respectively), plots of these geometries show quite different overall shapes; see Figure B.1.

For all fractals with volume fractal dimensions of 2.3, the mass enclosed in a sphere of size $S$ increases as $S^{2.3}$ and the density therefore varies as $S^{-0.7}$. The number 


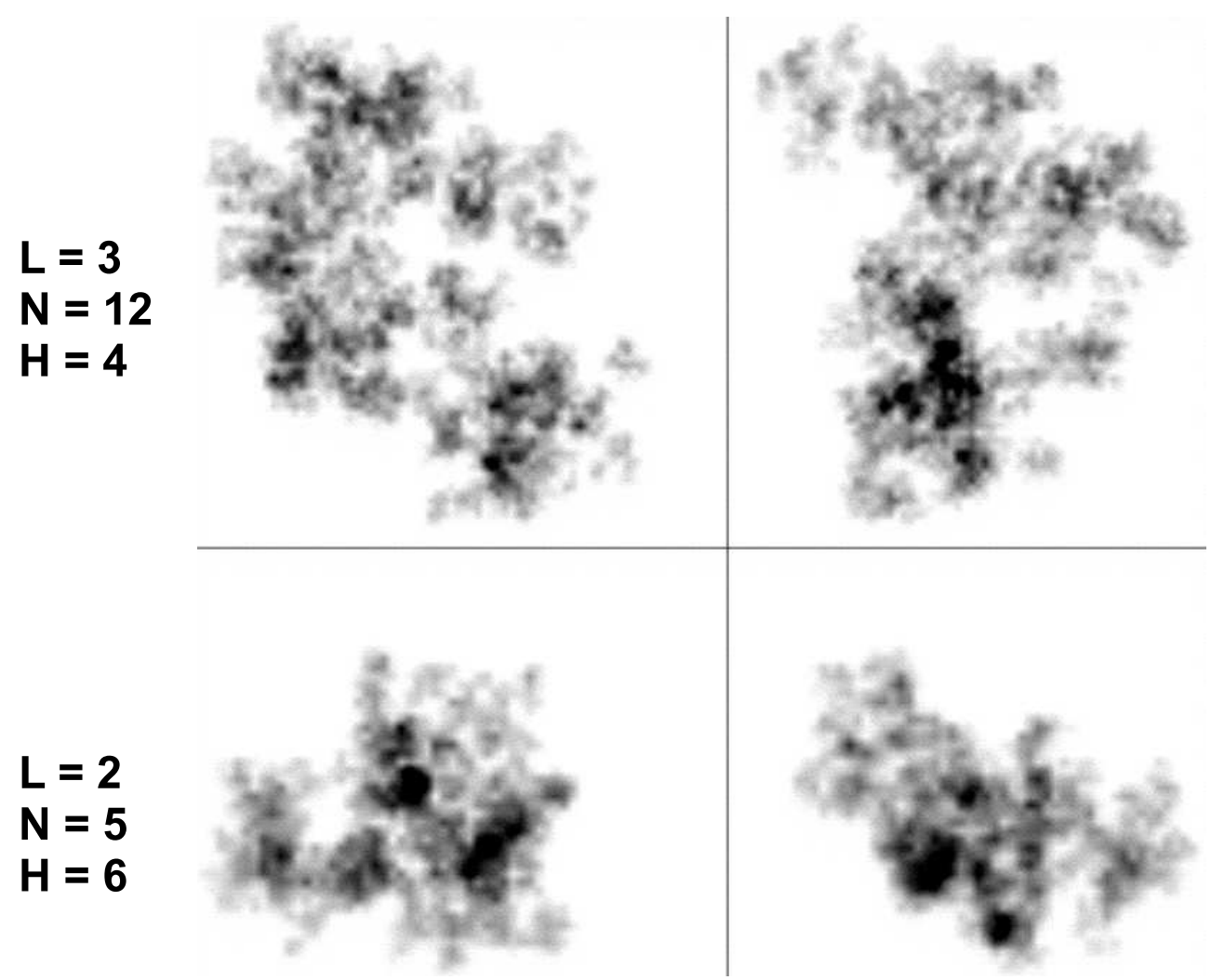

Figure B.1: Two views of two different three-dimensional fractal structures each with a fractal volume dimension value of 2.3 .

of clouds in the size interval $(S, S+d S)$ varies as $S^{-3.3}$, and the number of clouds with a mass in the interval $(M, M+d M)$ goes as $M^{-2}$. These relations are derived simply from the above relations.

The important thing for my research is that these fractal distributions allow a large fraction of sightlines to the central star cluster from all angles. The details, while physically important, are not yet known for several reasons. For one, young, embedded super star clusters are too far away to be resolved well enough with modern telescopes in order for measurements similar to those presented by Elmegreen [56] to be done. Just as importantly, however, is the fact that it is not known whether 
Appendix B. Appendix B: The Fractal Dust Distribution of a Super Star Cluster Embedding Envelope

the accumulated effects of supernovae, high-mass stellar winds, and radiation in super star cluster environments change the fractal geometry on relatively short timescales. We therefore simply fix the fractal dust distribution around super star clusters in Whelan et al. [218], owing to the fact that even slight changes to the volume fractal dimension produce relatively little overall noticeable change [90]. Furthermore, because super star cluster envelopes are believed to be quite large, we use a large value of $\mathrm{N}$. The following values are used to compute the fractal geometries in the super star cluster embedding envelopes: $N=32, L=3.792$, and $H=5$. These values produce a value for the volume fractal dimension of $D=2.6$, and a total of $N^{H}=3.36 \times 10^{7}$ points. 


\section{Appendix C}

\section{Molecular Line Radiative}

\section{Transfer with RATRAN}

\section{C.1 INTRODUCTION}

Models of molecular line excitation in more than one dimension call for Monte Carlo techniques because the trajectories are chosen based on random numbers, so in theory the method is faster than other methods [78]. Because geometry plays such an important rôle in the molecular line excitation, Hogerheijde \& van der Tak [78] chose to find the fastest Monte Carlo solution they could to predict molecular line excitation in star forming regions, and they settled on accelerated lambda iteration. Their equations are given below with the assumptions that I invoke for use on super star clusters to study CO and HCN emission. 


\section{C.2 Radiative Transfer and Molecular Excita- tion Coupling}

The equation of radiative transfer is

$$
\frac{d I_{\nu}}{d s}=-\alpha_{\nu} I_{\nu}+j_{\nu}
$$

and $d \tau_{\nu} \equiv \alpha_{\nu} d s$ and the source function $S_{\nu} \equiv \frac{\mathrm{J} \nu}{\alpha_{\nu}}$ so it can be re-written with those variables. $I_{\nu}$ is the intensity, $\nu$ is the frequency, $d s$ is an infinitesimal distance along the line of sight, $\alpha_{\nu}$ is the absorption coefficient $\left(\mathrm{cm}^{-1}\right)$ and $j_{\nu}$ is the emission coefficient ( $\mathrm{erg} \mathrm{s}^{-1} \mathrm{~cm}^{-3} \mathrm{~Hz}^{-1} \mathrm{sr}^{-1}$ ). Both molecules and dust particles are taken into account, so $j_{\nu}=j_{\nu}($ dust $)+j_{\nu}($ gas $)$ and $\alpha_{\nu}=\alpha_{\nu}($ dust $)+\alpha_{\nu}($ gas $)$.

The mean intensity is given by

$$
J_{\nu} \equiv \frac{1}{4 \pi} \int I_{\nu} d \Omega
$$

where $I_{\nu}$ is obtained by integrating the equation of radiative transfer. The outer boundary condition of this integration of the radiative transfer equation is given by the cosmic microwave background (CMB).

The dust is assumed to be thermally emitting, so $j_{\nu}(d u s t)=\alpha_{\nu}(d u s t) B_{\nu}\left(T_{d u s t}\right)$ and $\alpha_{\nu}($ dust $)=\kappa_{\nu} \rho_{\text {dust }}$ where $\kappa_{\nu}$ is the dust opacity $\left(\mathrm{cm}^{-2}\right.$ per unit dust mass $)$. I 
used a dust opacity given by Ossenkopf \& Henning [146] with thin ice mantles and no coagulation with time being the closest approximation to the dust opacities used in my dust radiative transfer codes from Kim et al. [105]. Furthermore, a gas-to-dust ratio of 100 is used, and the dust mass density $\rho_{\text {dust }}$ is computed from this and the given $\mathrm{H}_{2}$ envelope number densities.

Emission and absorption by the molecular line are computed assuming that local thermodynamic equilibrium (LTE) does not hold. $\alpha_{\nu}^{u l}$ (gas) and $\mathrm{j}_{\nu}^{u l}$ (gas) are therefore determined by absorption and emission between radiatively coupled levels $u$ and $l$ with level populations $n_{u}$ and $n_{l}$. The energy difference between levels correspond to the transition's frequency, which is the central frequency of a line-profile function $\phi(\nu)$. That makes for:

$$
\begin{gathered}
j_{\nu}^{u l}(\text { gas })=\frac{h \nu_{0}}{4 \pi} n_{u} A_{u l} \phi(\nu) \\
\alpha_{\nu}^{u l}(\text { gas })=\frac{h \nu_{0}}{4 \pi}\left(n_{l} B_{l u}-n_{u} B_{u l}\right) \phi(\nu)
\end{gathered}
$$

where the Einstein transition probabilities for spontaneous emission and absorption and stimulated emission are given by $A_{u l}, B_{l u}$, and $B_{u l}$. The line width is determined by Doppler broadening from the turbulent velocity field, so 


$$
\phi(\nu)=\frac{c}{b \nu_{0} \sqrt{\pi}} \exp \left(-\frac{c^{2}\left(\nu-\nu_{0}\right)^{2}}{\nu_{0}^{2} b^{2}}\right)
$$

where $b$ is the Gaussian full width at half maximum.

Level populations are given with collision rates that depend on the density and collisional rate coefficients. The collisional partners for $\mathrm{CO}$ are para- and ortho- $\mathrm{H}_{2}$, for $\mathrm{HCN}$ simply $\mathrm{H}_{2}$, and the collision rates and Einstein coefficients are obtained from the LAMDA database [176]. The level populations are thus found by solving

$$
n_{l}\left[\sum_{k<l} A_{l k}+\sum_{k \neq l}\left(B_{l k} J_{\nu}+C_{l k}\right)\right]=\sum_{k>l} n_{k} A_{k l}+\sum_{k \neq l} n_{k}\left(B_{k l} J_{\nu}+C_{k l}\right) .
$$

$J_{\nu}$ depends on the CMB, the dust and the spectral line, so it can't be known accurately on the first pass and $J_{\nu}$ has to be solved iteratively. RATRAN then solves for the level populations by matrix inversion for each radius.

\section{C.3 Finding $J_{\nu}$ using the $\Lambda$-operator}

$J_{\nu}$ is built up in each radial grid cell by summing up the emission received in cell $i$ from all other grid cells $j$ after propagation through the intervening grid cells. The radiation field can therefore be parameterized as an operator acting on the source function, which is also dependent on the radiation field. In maths jargon, 


$$
J_{\nu}=\Lambda\left[S_{u l}\left(J_{\nu}\right)\right]
$$

where the source function $S_{\nu}$ has been replaced by

$$
S_{u l}=\frac{j_{\nu 0}(d u s t)+\int j_{\nu}^{u l}(\text { gas }) d \nu}{\alpha_{\nu 0}(d u s t)+\int \alpha_{\nu}^{u l}(\text { gas }) d \nu}
$$

on account of the consideration of the narrow frequency range around $\nu_{0}$. After an initial guess, $J_{\nu}$ can be solved using the source function from the previous iteration, $S_{u l}^{\dagger}$.

The Monte Carlo part of the code assumes that CMB photons are propagating through the gas and dust from random directions but only considers CMB and interacting photons that converge on the grid cell in question. (Scattering is not included in the code because it is believed not to be important longer than about $10 \mu \mathrm{m}$ in wavelength.) However, there is a snag at large optical depths. The photons passing through high optical depth grid cells quickly tend toward the local source function after several interactions on small mean free paths, and the number of iterations grow $\propto \tau^{2}$. To get around this, they use Accelerated Lambda Iteration that defines an approximate operator $\Lambda^{*}$ which is easily invertible. Using $\Lambda^{*}$ can steer rapidly toward convergence if it is the diagonal part of the full operator $\Lambda$. This describes the generated field in each grid cell. $J_{\nu}$ can be solved then as 


$$
J_{\nu}=\left(\Lambda-\Lambda^{*}\right)\left[S_{u l}^{\dagger}\left(J_{\nu}\right)\right]+\Lambda^{*}\left[S_{u l}\left(J_{\nu}\right)\right]
$$

Sub-iterations are used to separate $J_{\nu}$ into locally generated and external (i.e. CMB and other grid cells) contributions so that $S_{u l}$ and $\tau$ can be solved locally. For ray $i, I_{0, i}$ is the incident radiation field on the cell and $d s_{i}$ the distance to the boundary cell, which translates into $\tau_{i}$ based on the current level populations. After $N$ rays, the average radiation field is just

$$
J_{\nu}=\frac{1}{N} \sum_{i} I_{0, i} e^{-\tau_{i}}+\frac{1}{N} \sum_{i} S_{u l}\left[1-e^{-\tau_{i}}\right]=J_{\nu}^{\text {external }}+J_{\nu}^{\text {local }}
$$

For low optical depths, the implementation of the $\Lambda^{*}$ operator does nothing to speed up the process, nor does it hurt. But at high optical depths, the time efficiency is significant.

\section{C.4 Outline of Code}

From Hogerheijde \& van der Tak [78] Appendix A, the outline of how the code progresses and eventually converges on a solution is given here.

1. Input and compile 
(a) Calculation parameters in the inp file including number of photons $\mathrm{N}$ and desired $\mathrm{S} / \mathrm{N}$ value

(b) The source model ([source].mdl file)

(c) Molecular data from the LAMDA project's website

(d) Compile the code

2. Find $J_{\nu}$ iteratively for a fixed set of photons

(a) With N, start at a random position and propagate to both cell boundary and edge of cloud to determine $J_{\nu}$

(b) Iterate on level populations and $J_{\nu}$, converging to an accuracy of $10^{-6}$

(c) If the last three solutions have a difference of $<1 / 10$ the required accuracy, then continue

3. Find $J_{\nu}$ iteratively for a variable and increasing number of photons

(a) With $\mathrm{N}$ or $2 \times$ the last highest number of photons, start in a random direction and propagate to the cell boundary and edge of cloud to determine $J_{\nu}$

(b) Iterate on level populations and $J_{\nu}$, converging to an accuracy of $10^{-6}$

(c) Compare the last three solutions. If the difference between them is greater than the required accuracy, then reiterate with twice the number of photons of the last iteration. It the difference is less than the required accuracy, then continue 
4. Write output

After the program outputs its results, a second program uses the output of the first to determine how the resolved source would look on the sky. This second program can be used to zoom in on the central portion as much as desired. 


\section{Bibliography}

[1] Abel, N. P., Ferland, G. J., Shaw, G., \& van Hoof, P. A. M. 2005, ApJS, 161, 65.

[2] Allamandola, L. J., Tielens, A. G. G. M., \& Barker, J. R. 1989, ApJS, 71, 733.

[3] Arp, H., \& Sandage, A. 1985, AJ, 90, 1163.

[4] Ashman, K. M. \& Zepf, S. E. 2001, AJ, 122, 1888.

[5] Aversa, A. G., Johnson, K. E., Brogan, C. L., Goss, W. M., \& Pisano, D. J. 2011, AJ, 141, 125.

[6] Baker, J. G., \& Menzel, D. H. 1938, ApJ, 88, 52.

[7] Baker, J. G., Menzel, D. H., \& Aller, L. H. 1938, ApJ, 88, 422.

[8] Bakes, E. L. O., \& Tielens, A. G. G. M. 1994, ApJ, 427, 822.

[9] Beck, S.C., Turner, J. L., Langland-Shula, L. E., Meier, D. S., Crosthwaite, L. P., Gorjian, V. 2002, AJ, 124, 2516.

[10] Benjamin, R. A.,Churchwell, E., Babler, B. L., et al. 2003, PASP, 115, 953. 
[11] van den Bergh, S. 1971, A\&A, 12, 474.

[12] Bernard-Salas, J., Spoon, H. W. W., Charmandaris, V., Lebouteiller, V., Farrah, D., Devost, D., Brandl, B. R., Wu, Y., Armus, L., Hao, L., Sloan, G. C., Weedman, D., \& Houck, J. R. 2009, ApJS, 184, 230.

[13] Bernard-Salas, J., Peeters, E., Sloan, G. C., et al. 2009, ApJ, 699, 1541.

[14] Bisbas, T. G., Bell, T. A., Viti, S., Yates, J., \& Barlow, M. J. 2012, MNRAS, 427, 2100.

[15] Bonnell, I. A., Smith, R. J., Clark, P. C., \& Bate, M. R. 2010, MNRAS, in press. arXiv:1009.1152.

[16] Bosch, G., Terlevich, E., \& Terlevich, R. 2009, AJ, 137, 3437.

[17] Bouret, J.-C., Lanz, T., Hillier, D. J., Heap, S. R., Hubeny, I., Lennon, D. J., Smith, L. J., \& Evans, C. J. 2003, ApJ, 595, 1182.

[18] Brandl, B. R., Bernard-Salas, J., Spoon, H. W. W., Devost, D., Sloan, G. C., Guiles, S., Wu, Y., Houck, J. R., Weedman, D. W., Armus, L., Appleton, P. N., Soifer, B. T., Charmandaris, V., Hao, L., Higdon, J. A., Marshall, S. J., \& Herter, T. L. 2006, ApJ, 653, 1129.

[19] Brinch, C., \& Hogerheijde, M. R. 2010, A\&A, 523, 25.

[20] Brocklehurst, M. 1971, MNRAS, 153, 471.

[21] Brogan, C., Johnson, K., \& Darling, J. 2010, ApJ, 716, 51. 
[22] Calzetti, D., Kennicutt, R. C., Engelbracht, C. W., et al. 2007, ApJ, 666, 870.

[23] Caplan, J., Ye, T., Deharveng, L., Turtle, A. J., \& Kennicutt, R. C. 1996, A\&A, 307, 403.

[24] Cardelli, J. A., Clayton, G. C., \& Mathis, J. S. 1989, ApJ, 345, 245.

[25] Castelli Kurucz. IAU Symposium 210, Modelling of Stellar Atmospheres, Uppsala, Sweden, eds. N.E. Piskunov, W.W. Weiss. and D.F. Gray, 2003, ASPS210.

[26] Clark, J. S., Negueruela, I., Crowther, P. A., \& Goodwin, S. P. 2005, A\&A, 434,949 .

[27] Chakrabarti, S. \& Whitney, B. A. 2009, ApJ, 690, 1432.

[28] Chamberlain, J. W. 1953, ApJ, 117, 387.

[29] Chandar, R., Fall, S. M., \& Whitmore, B. C. 2010, ApJ, 711, 1263.

[30] Chevalier, R. A., \& Clegg, A. W. 1985, Nature, 317, 44.

[31] Chiar, J. E., Ennico, K., Pendleton, Y. J., Boogert, A. C. A., Greene, T., Knez, C., Lada, C., Roellig, T., Tielens, A. G. G. M., Werner, M., \& Whittet, D. C. B. 2007, ApJ, 666, L73.

[32] Churchwell, E., \& Goss, W. M. 1999, ApJ, 514, 188.

[33] Churchwell, E. 2002, ARA\&A, 40, 27. 
[34] Churchwell, E., Whitney, B. A., Babler, B. L., Indebetouw, R., Meade, M. R., Watson, C. Wolff, M. J., Wolfier, M. G., Bania, T. M., Benjamin, R. A., Clemens, D. P., Cohen, M., Devine, K. E., Dickey, J. M., Heitsch, F., Jackson, J. M., Kobulnicky, H. A., Marston, A. P., Mathis, J. S., Mercer, E. P., Stauffer, J. R., \& Stolovy, S. R. 2004, ApJS, 154, 322.

[35] Churchwell, E.,Babler, B. L., Meade, M. R., et al. 2009, PASP, 121, 213.

[36] Compiègne, M., Abergel, A., Verstraete, L., Reach, W. T., Habart, E., Smith, J. D., Boulanger, F., \& Joblin, C. 2007, A\&A, 471, 205.

[37] Conti, P. S., \& Vacca, W. D. 1994, ApJ, 423, L97

[38] Contursi, A., Lequeux, J., Cesarsky, D., Boulanger, F., Rubio, M., Hanus, M., Sauvage, M., Tran, D., Bosma, A., Madden, S., \& Vigroux, L. 2000, A\&A, 362,310 .

[39] Cornette, W. M. \& Shanks, J. G. 1992 Applied Optics, 31, 3152

[40] Cox, N. J. L., Cordiner, M. A., Ehrenfreund, P., Kaper, L., Sarre, P. J., Foing, B. H., Spaans, M., Cami, J., Sofia, U. J., Clayton, G. C., Gordon, K. D., \& Salama, F. 2007, A\&A, 470, 941.

[41] Crutcher, R. M., Wandelt, B., Heiles, C., Falgarone, E., \& Troland, T. H. 2010, ApJ, 725, 466.

[42] Crutcher, R. M. 2012, ARA\&A, 50, 29. 
[43] Decressin, T., Baumgardt, H., Charbonnel, C., \& Kroupa, P. 2010, A\&A, $516,73$.

[44] can Diedenhoven, B., Peeters, E., Van Kerckhoven, C., Hony, S., Hudgins, D. M., Allamandola, L. J., \& Tielens, A. G. G. M. 2004, ApJ, 611, 928.

[45] Dopita, M. A., Groves, B. A., Sutherland, R. S., \& Kewley, L. J. 2003, ApJ, 583,727 .

[46] Dopita, M. A., Groves, B. A., Fischera, J., et al. 2005, ApJ, 619, 755.

[47] Dopita, M. A., Fischera, J., Sutherland, R. S., et al. 2006, ApJ, 647, 244.

[48] Draine, B. T. 2010: arXiv:1004.0474.

[49] Draine, Bruce, T. Physics of the Interstellar and Intergalactic Medium. Princeton: Princeton University Press, 2011.

[50] Draine, B. T.,Dale, D. A., Bendo, G., Gordon, K. D., Smith, J. D. T., Armus, L., Engelbracht, C. W., Helou, G., Kennicutt, R. C., Li, A., Roussel, H., Walter, F., Calzetti, D., Moustakas, J., Murphy, E. J., Rieke, G. H., Bot, C., Hollenbach, D. J., Sheth, K. \& Teplitz, H. I. 2007, ApJ, 663, 866

[51] Draine, B. T., \& Li, A. 2007, ApJ, 657, 810

[52] Dufour, R. J., \& Harlow, W. V. 1977, ApJ, 216, 706.

[53] Ekta, B., Pustilnik, S. A., \& Chengalur, J. N. 2009, MNRAS, 397, 963.

[54] Eldridge, J. J., \& Relaño, M. 2010, MNRAS, submitted. arXiv:1009.1871. 
[55] Elmegreen, B. G., \& Efremov, Y. N. 1996, ApJ, 466, 802.

[56] Elmegreen, B. G. 1997, ApJ, 477, 196.

[57] Elmegreen, B. G., \& Efremov, Y. N. 1997, ApJ, 480, 235.

[58] Fazio, G. G. et al. 2004, ApJS, 154, 10.

[59] Ferguson, J. W., \& Ferland, G. J. 1997, ApJ, 479, 363.

[60] Ferland, G. J. 1995, The Analysis of Emission Lines Conference. eds. R. Williams \& M. Livio, Cambridge University Press, p. 83.

[61] Ferland, G. J. 1999, PASP, 111, 1524.

[62] Ferland, G. J., Korista, K. T., Verner, D. A., Ferguson, J. W., Kingdon, J. B., \& Verner, E. M. 1998, PASP, 110, 761.

[63] Furlan, E., Luhman, K. L., Espaillat, C., D’Alessio, P., Adame, L., Manoj, P., Kim, K. H., Watson, D. M., Forrest, W. J., McClure, M. K., Calvet, N., Sargent, B. A., Green, J. D., \& Fischer, W. J. 2011, ApJS, 195, 3.

[64] Galliano, F., Madden, S. C., Tielens, A. G. G. M., Peeters, E., \& Jones, A. P. 2008, ApJ, 679, 310.

[65] Gao, Y., \& Solomon, P. M. 2004, ApJ, 606, 271.

[66] Gouliermis, D. A., Dolphin, A. E., Bradner, W., \& Henning, T. 2006, ApJS, 166,549 . 
[67] Gouliermis, D. A., Bestenlehner, J. M., Bradner, W., \& Henning, T. 2010, A\&A, 515, 56.

[68] Hansen, C. J., Kawaler, S. D., \& Trimble, V. Stellar Interiors: Physical Principles, Structure, and Evolution. New York: Springer, 2004.

[69] Hao, L., Spoon, H. W. W., Sloan, G. C., Marshall, J. A., Armus, L., Tielens, A. G. G. M., Sargent, B., van Bemmel, I. M., Charmandaris, V., Weedman, D. W., \& Houck, J. R. 2005, ApJ, 625, 75.

[70] Haser, S. M., Pauldrach, A. W. A., Lennon, D. J., Kudritzki, R.-P., Lennon, M., Puls, J., \& Voels, S. A. 1998, A\&A, 330, 285.

[71] Heitsch, F., Whitney, B. A., Indebetouw, R., Meade, M. R., Babler, B. L., \& Churchwell, E. 2007, ApJ, 656, 227.

[72] Heitsch, F., Naab, T., \& Walch, S. 2011, MNRAS, 415, 271.

[73] Henize, K. G. 1956, ApJS, 2, 315.

[74] Henyey, L. G., \& Greenstein, J. L. 1941, ApJ, 93, 70.

[75] Heydari-Malayeri, M., \& Selier, R. 2010, A\&A, 517, A39.

[76] Higdon, S. J. U., Devost, D., Higdon, J. L., Brandl, B. R., Houck, J. R., Hall, P., Barry, D., Charmandaris, V., Smith, J. D. T., Sloan, G. C., \& Green, J. 2004, PASP, 116, 975.

[77] Hilditch, R. W., Howarth, I. D., \& Harries, T. J. 2005, MNRAS, 357, 304. 
[78] Hogerheijde, M. R., \& van der Tak, F. F. S. 2000, A\&A, 362, 697.

[79] Holtzman, J. A., Faber, S. M., Shaya, E. J., Lauer, T. R., Groth, E. J., Hunter, D. A., Baum, W. A., Ewald, S. P., Hester, J. J., Light, R. M., Lynds, C. R., O'Neil, E. J., \& Westphal, J. A. 1992, AJ, 103, 691.

[80] Hony, S., Van Kerckhoven, C., Peeters, E., Tielens, A. G. G. M., Hudgins, D. M., \& Allamandola, L. J. 2001, A\&A, 370, 1030.

[81] Houck, J. R., et al. 2004, ApJS, 154, 18.

[82] Houck, J. R., Charmandaris, V., Brandl, B. R., Weedman, D., Herter, T., Armus, L., Soifer, B. T., Bernard-Salas, J., Spoon, H. W. W., Devost, D., \& Uchida, K. I. 2004, ApJ, 154, 211.

[83] Hubeny, I., Lanz, T. 1995, ApJ, 439, 875.

[84] Hudgins, D. M., \& Allamandola, L. J. 1999, ApJ, 516, 41.

[85] Hummer, D. G., Storey, P. J. 1987, MNRAS, 224, 801.

[86] Hunt, L. K., Vanzi, L., \& Thuan, T. X. 2001, A\&A, 377, 66.

[87] Hunt, L., Bianchi, S., \& Maiolino, R. 2005, A\&A, 434, 849.

[88] Hunt, L. K., Thuan, T. X., Izotov, Y. I., \& Sauvage, M. 2010, ApJ, 712, 164.

[89] Hunter, D. A., Shaya, E. J., Scowen, P., Hester, J. J., Groth, E. J., Lynds, R. \& O’Neil, E. J. 1995, ApJ, 444, 758. 
[90] Indebetouw, R., Whitney, B. A., Johnson, K. E, \& Wood, K. 2006, ApJ, 636, 362.

[91] Indebetouw, R., de Messières, G. E., Madden, S., Engelbracht, C., Smith, J. D., Meixner, M., Brandl, B., Smith, L. J., Boulanger, F., Galliano, F., Gordon, K., Hora, J. L., Sewilo, M., Tielens, A. G. G. M., Werner, M., \& Wolfire, M. G. 2009, ApJ, 694, 84.

[92] Izotov, Y. I., Guseva, N. G., Lipovetsky, V. A., Kniazev, A. Y., \& Stepanian, J. A. 1990, Nature, 343, 238.

[93] Izotov, Y. I., Chaffee, F. H., \& Schaerer, D. 2001, A\&A, 378, 45.

[94] Izotov, Y. I., \& Thuan, T. X. 2004, ApJ, 602, 200.

[95] Izotov, Y. I., Guseva, N. G., Fricke, K. J., \& Papaderos, P. 2009, A\&A, 503, 61.

[96] Izotov, Y. I, \& Thuan, T. X. 2011, ApJ, 734, 82.

[97] Johnson, K. E., Vacca, W. D., Leitherer, C., Conti, P. S., \& Lipsky, S. J. 1999, AJ, 117, 1708.

[98] Johnson, K. E., Leitherer, C., Vacca, W. D., \& Conti, P. S. 2000, AJ, 120, 1273.

[99] Johnson, K. E., Kobulnicky, H. A., Massey, P., \& Conti, P. S. 2001, ApJ, 559, 864. 
[100] Johnson, K. E., Indebetouw, R., \& Pisano, D. J. 2003, AJ, 126, 101.

[101] Johnson, K. E. \& Kobulnicky, H. A. 2003, ApJ, 597, 923.

[102] Johnson, K. E., Hunt, L. K., Reines, A. E. 2009, AJ, 137, 3788.

[103] Keller, L. D., Sloan, G. C., Forrest, W. J., Ayala, S., D’Alessio, P., Shah, S., Calvet, N., Najita, J., Li, A., Hartmann, L., Sargent, B., Watson, D. M., \& Chen, C. H. 2008, ApJ, 684, 411.

[104] Kennicutt, R. C. 1984, ApJ, 287, 116.

[105] Kim, S.-H., Martin, P. G., \& Hendry, P. D. 1993, ApJ, 422, 164.

[106] Kim, Y., Rieke, G. H., Krause, O., Misselt, K., Indebetouw, R., \& Johnson, K. E. 2008, ApJ, 678, 287.

[107] Klessen, R. S., Heitsch, F., \& Mac Low, M.-M. 2000, ApJ, 535, 887.

[108] Kobulnicky, H. A. \& Johnson, K. E. 1999, ApJ, 527, 154.

[109] Kornei, K. A., \& McCrady, N. 2009, ApJ, 697, 1180.

[110] Kroupa, P., \& Weidner, C. 2003, ApJ, 598, 1076.

[111] Kurtz, S., Churchwell, E., Wood, D. O. S. 1994, ApJS, 91, 659.

[112] Laor, A. \& Draine, B. T. 1993, ApJ, 402, 441.

[113] Lazarian, A., Esquivel, A., \& Crutcher, R. 2012, ApJ, 757, 154. 
[114] Lebouteiller, V., Brandl, B., Bernard-Salas, J., Devost, D., \& Houck, J. R. 2007, ApJ,, 665, 390.

[115] Lebouteiller, V., Bernard-Salas, J., Brandl, B., Whelan, D. G., Wu, Yanling, Charmandaris, V., Devost, D., \& Houck, J. R. 2008, ApJ, 680, 398.

[116] Lebouteiller, V., Bernard-Salas, J., Sloan, G. C., \& Barry, D. J. 2010, PASP, $122,231$.

[117] Lebouteiller, V., Bernard-Salas, J., Whelan, D. G., Brandl, B., Galliano, F., Charmandaris, V., Madden, S., \& Kunth, D. 2011, ApJ, 728, 45.

[118] Lebouteiller, V., Sloan, G. C., Groenewegen, M. A. T., Matsuura, M., Riebel, D., Whelan, D. G., Bernard-Salas, J., \& Massey, P. 2012, A\&A, 546, 94.

[119] Leger, A., \& Puget, J. L. 1984, AA, 137, L5.

[120] Leitherer, C., Schaerer, D., Goldader, J. D., González Delgado, R. M., Robert, C., Kune, D. F., de Mello, D. F., Devost, D., Heckman, T. M. 1999, ApJS, 123, 3.

[121] Li, A., \& Draine, B. T. 2001, ApJ, 554, 778.

[122] van Loon, J. T., Oliveira, J. M., Wood, P. R., Zijlstra, A. A., Sloan, G. C., Matsuura, M., Whitelock, P. A., Groenewegen, M. A. T., Blommaert, J. A. D. L., Cioni, M.-R. L., Hony, S., Loup, C., \& Waters, L. B. F. M. 2005, MNRAS, 364, L71. 
[123] Lucy, L. B. 1999, A\&A, 344, 282.

[124] Luridiana, V., Simón-Díaz, S., Cerviño, M., González Delgado, R. M., Porter, R. L., \& Ferland, G. J. 2009, ApJ, 691, 1712.

[125] Mac Low, M.-M., \& McCray, R. 1988, ApJ, 324, 776.

[126] Madden, S. C., Galliano, F., Jones, A. P., Sauvage, M. 2006, A\&A, 446, 877.

[127] de Marchi, G., Clampin, M., Greggio, L., Leitherer, C., Nota, A., \& Tosi, M. 1997, ApJL, 479, L27.

[128] Massey, P., Parker, J. W., \& Garmany, C. D. 1989, AJ, 98, 1305.

[129] Massey, P., \& Hunter, D. A. 1998, ApJ, 493, 180.

[130] Massey, P., Puls, J., Pauldrach, A. W. A., Bresolin, F., Kudritzki, R. P., \& Simon, T. 2005, ApJ, 627, 477.

[131] Maybhate, A., Masiero, J., Hibbard, J. E., Charlton, J. C., Palma, C., Knierman, K. A., \& English, J. 2007, MNRAS, 381, 59.

[132] McCray, R., \& Kafatos, M. 1987, ApJ, 317, 190.

[133] McKee, C. E., \& Ostriker, J. P. 1977, ApJ, 218, 148.

[134] Melnick, J., Heydari-Malayeri, M., \& Leisy, P. 1992, A\&A, 253, 16.

[135] Moffat, A. F. J. 1982, JRASC, 76, 323. 
[136] Mouschovias, T. M., \& Ciolek, G. E. 1999, The Origins of Stars and Planetary Systems, 305.

[137] Moustakas, J., Kennicutt, R. C. Jr., Tremonti, C. A., Dale, D. A., Smith, J. D. T., \& Calzetti, D. 2010, ApJS, 190, 233.

[138] Murray, N., Quataert, E., \& Thompson, T. A. 2010, ApJ, 709, 191.

[139] Murray, N., Menard, B., \& Thompson, T. A. 2010, ApJ, submitted. arXiv:1005.4419

[140] Myers, P. C., Adams, F. C., Chen, H., \& Schaff, E. 1998, ApJ, 492,703.

[141] O'Connell, R. W., Gallagher, J. S., \& Hunter, D. A. 1994, 433, 65

[142] O'Connell, R. W., Gallagher, J. S., Hunter, D. A., \& Colley, W. N. 1995, ApJL, 446, 1.

[143] Oey, M. S., \& Clarke, C. J. 1997, MNRAS, 289, 570.

[144] Olive, K. A., \& Skillman, E. D. 2004, ApJ, 617, 29.

[145] Oliveira, V. A., Copetti, M. V. F., \& Krabbe, A. C. 2008, A\&A, 492, 463.

[146] Ossenkopf, V., \& Henning, T. 1994, A\&A, 291, 943.

[147] Osterbrock, D. E., \& Ferland, G. J. Astrophysics of Gaseous Nebulae and Active Galactic Nuclei, Second Edition. Sausalito: University Science Books, 2006. 
[148] Parker, J. W. 1993, ApJ, 106, 560.

[149] Peeters, E., Hony, S., Van Kerckhoven, C., Tielens, A. G. G. M., Allamandola, L. J., Hudgins, D. M., \& Bauschlicher, C. W. 2002, A\&A, 390, 1089.

[150] Peeters, E., Spoon, H. W. W., \& Tielens, A. G. G. M. 2004, ApJ, 613, 986.

[151] Peimbert, M., Peimbert, A., \& Ruiz, M. T. 2000, ApJ, 541, 688.

[152] Peimbert, A., Peimbert, M., \& Luridiana, V. 2002, ApJ, 565, 668.

[153] Pellegrini, E. W., Baldwin, J. A., \& Ferlan, G. J. 2011, ApJ, 738, 34.

[154] Pengelly, R. M. 1964, MNRAS, 127, 145.

[155] Pirogov, L., Zinchenko, I, Lapinov, A., Myshenko, V., \& Shul'Ga, V. 1995, A\&AS, 109, 333.

[156] Plante, S., \& Sauvage, M. 2002, AJ, 124, 1995.

[157] Puget, J. L., \& Léger, A. 1989, ARA\&A, 27, 161.

[158] Pustilnik, S. A., Brinks, E., Thuan, T. X., Lipovetsky, V. A., \& Izotov, Y. I. 2001, AJ, 121, 1413.

[159] Reines, A. E., Johnson, K. E., \& Hunt, L. K. 2008, AJ, 135, 2222.

[160] Rieke, G., et al. 2004, ApJS, 154, 25.

[161] Robberto, M., Bechwith, S. V. W., Panagia, N., Patel, S. G., Herbst, T. M., Ligori, S., Custo, A., Boccacci, P., \& Bertero, M. 2005, AJ, 129, 1534. 
[162] Robinson, G., \& Maldoni, M. M. 2010, MNRAS, 408, 1956.

[163] Robitaille, T. P., Whitney, B. A., Indebetouw, R., Wood, K., \& Denzmore, P. 2006, ApJS, 167, 256.

[164] Robitaille, T. P., Whitney, B. A., Indebetouw, R., \& Wood, K. 2007, ApJS, 169,328 .

[165] Rollig, M., Abel, N. P., Bell, T., Bensch, F., Black, J., Ferland, G. J., Jonkheid, B., Kamp, I., Kaufman, M. J., Le Bourlot, J., Le Petit, F., Meijerint, R., Morata, O., Ossenkopf, V., Roueff, E., Shaw, G., Spaans, M., Sternberg, A., Stutzki, J., Thi, W.-F., van Dishoeck, E. F., van Hoof, P. A. M., Viti, S., \& Wolfire, M. G. 2007, A\&A, 467, 187.

[166] Roussel, H., Helou, G., Hollenbach, D. J., Draine, B. T., Smith, J. D., Armus, L., Schinnerer, E., Walter, F., Engelbracht, C. W., Thornley, M. D., Kennicutt, R. C., Calzetti, D., Dale, D. A., Murphy, E. J., \& Bot, C. 2007, ApJ, 669, 959.

[167] Rubio, M., Contursi, A., Lequeux, J., Probst, R., Barbá, R., Boulanger, F., Cesarsky, D., \& Maoli, R. 2000, A\&A, 359, 1139.

[168] Russell, S. C., \& Dopita, M. A. 1992, ApJ, 384, 508.

[169] Sabbi, E., Sirianni, M., Nota, A., Tosi, M., Gallagher, J., Meixner, M., Oey, M. S., Walterbos, R., Pasquali, A., Smith, L. J., \& Angeretti, L. 2007, AJ, 133, 44. 
[170] Sabbi, E., Sirianni, M., Nota, A., Tosi, M., Gallagher, J., Smith, L. J., Angeretti, L., Meixner, M., Oey, M. S., Walterbos, R., \& Pasquali, A. 2008, AJ, 135,173 .

[171] Salpeter, E. E. 1955, ApJ, 121, 161.

[173] Sandstrom, K. M., Bolatto, A. D., Draine, B. T., Bot, C., \& Stanimirović, S. 2010, ApJ, 715, 701.

[173] Sandstrom, K. M., Bolatto, A. D., Draine, B. T., Bot, C., \& Stanimirović, S. 2010, ApJ, 715, 701 .

[174] Sandstrom, K. M., Bolatto, A. D., Bot, C., Draine, B. T., Ingalls, J. G., Israel, F. P., Jackson, J. M., Leroy, A. K., Li, A., Rubio, M., Simon, J. D., Smith, J. D. T., Stanimirović, S., Tielens, A. G. G. M., \& van Loon, J. 2012, ApJ, 744, 20.

[175] Sauvage, M., \& Plante, S. 2003, Ap\&SS, 284, 941.

[176] Schöier, F., L., van der Tak, F. F. S., van Dishoeck, E. F., \& Black, J. H. 2005, A\&A, 432, 369.

[177] Scowen, P. A., Hauschildt, P. H., Aufdenberg, J. P., \& Sankrit, R. 1997, AIPC, 393, 291.

[178] Schutte, W. A., Tielens, A. G. G. M., \& Allamandola, L. J. 1993, ApJ, 415, 397. 
[179] Schweizer, F., Miller, B. Y., Whitmore, B. C., \& Fall, S. M. 1996, AJ, 112, 1839

[180] Seaton, M. J. 1959, MNRAS, 119, 81.

[181] Seaton, M. J. 1959, MNRAS, 119, 90.

[182] Seaton, M. J. 1960, RPPh, 23, 313.

[183] Shahbazian, R. K. 1970, Astrofisika, 6, 367.

[184] Shioya, Y., Taniguchi, Y., \& Trentham, N. 2001, MNRAS, 321, 11.

[185] Shu, F. H. 1977, ApJ, 214, 488.

[186] Silich, S., Tenorio-Tagle, G., \& Muñoz-Tuñon, G. 2003, ApJ, 590, 791.

[187] Silich, S., Tenorio-Tagle, G., \& Rodriguez-Gonzalez, A. 2004, ApJ, 610, 226.

[188] Sicilia-Aguilar, A., Hartmann, L. W., Watson, D., Bohac, C., Henning, T., \& Bouwman, J. 2007, ApJ, 659, 1637.

[189] Simon, J. D., Bolatto, A. D., Whitney, B. A., Robitaille, T. P., Shah, R. Y., Makovoz, D., Stanimirović, S., Barbá, R. H., \& Rubio, M. 2007, ApJ, 669, 327.

[190] Simon, M., Felli, M., Cassar, L., Fischer, J., Massi, M. 1983, ApJ, 266, 623.

[191] Sloan, G. C., \& Price, S. D. 1995, ApJ, 451, 758.

[192] Sloan, G. C., Keller, L. D., Forrest, W. J., et al. 2005, ApJ, 632, 956. 
[193] Smith, J. D. T., Draine, B. T., Dale, D. A., Moustakas, J., Kennicutt, R. C., Helou, G., Armus, L., Roussel, H., Sheth, K., Bendo, G. J., Buckalew, B. A., Calzetti, D., Engelbracht, C. W., Gordon, K. D., Hollenback, D. J., Li, A., Malhotra, S., Murphy, E. J., Walter, F. 2007, ApJ, 656, 770.

[194] Sonneborn, G. 2008, IAU Symposium 250, 491

[195] Spitzer, L. J. Physical Processes in the Interstellar Medium. New York, Wiley, 1978.

[196] Stahler, S. W. 2012, PT, 65, 10, 46.

[197] Storey, P. J., \& Hummer, D. G. 1995, MNRAS, 272, 41.

[198] Sturm, E., Schweitzer, M., Lutz, D., Contursi, A., Genzel, R., Lehnert, M. D., Tacconi, L. J., Veilleux, S., Rupke, D. S., Kim, D.-C., Sternberg, A., Maoz, D., Lord, S., Mazzarella, J., Sanders, D. B. 2005, ApJ, 629, 21.

[199] Tacconi-Garman, L. E., Sternberg, A., \& Eckart, A. 1996, AJ, 112, 918.

[200] van der Tak, F. F. S., Black, J. H., Schöier, F. L., Jansen, D. J., \& van Dishoeck, E. F. 2007, A\&A, 468, 627.

[201] Tenorio-Tagle, G., Silich, S., Rodríguez-González, A., Muñoz-Tuñón, C. 2005, ApJ, 620, 217.

[202] Tenorio-Tagle, G., Wünsch, R., Silich, S., Palouš, J. 2007, ApJ, 658, 1196. 
[203] Thompson, R. I., Sauvage, M., Kennicutt, R. C. Jr., Engelbracht, C. W., \& Vanzi, L. 2006, ApJ, 638, 176.

[204] Thuan, T. X. 2008, IAUS, 255, 348.

[205] Thuan, T. X., Lecavelier des Etangs, A., \& Izotov, Y. I. 2005, ApJ, 621, 269.

[206] Tielens, A. G. G. M., Hony, S., van Kerckhoven, C., \& Peeters, E. 1999, in The Universe as Seen by ISO, ed. P. Cox \& M. F. Kessler (ESA SP-427; Nordwijk; ESA), 579.

[207] Tielens, A. G. G. M. The Physics and Chemistry of the Interstellar Medium. Cambridge: Cambridge University Press, 2005.

[208] Troland, T. H., \& Crutcher, R. M. 2008, ApJ, 680, 457.

[209] Tsamis, Y. G., Barlow, M. J., Liu, X.-W., Danziger, I. J., \& Storey, P. J. 2003, MNRAS, 338, 687.

[210] Turner, J. L., Beck, S. C., \& Ho, P. T. P. 2000, ApJ, 532, 109.

[211] Vacca, W. D., Johnson, K. E., \& Conti, P. S. 2002, AJ, 123, 772.

[212] Vermeij, R., Peeters, E., Tielens, A. G. G. M., \& van der Hulst, J. M. 2002, A\&A, 382, 1042.

[213] Verschuur, G. L. 1995, Ap\&SS, 227, 187. 
[214] Verdolini, S., Yeh, S. C. C., Krumholz, M. R., Matzner, C. D., \& Tielens, A. G. G. M. 2013, ApJ, accepted. arXiv:1303.4737.

[215] Walborn, N. R., \& Blades, J. C. 1986, ApJ, 304, L17.

[216] Warren, S. G. 1984, AO, 23, 1206

[217] Weaver, R., McCray, R., Castor, J., Shapiro, P., \& Moore, R. 1977, ApJ, 218,377 .

[218] Whelan, D. G., Johnson, K. E., Whitney, B. A., Indebetouw, R., \& Wood, K. 2011, ApJ, 729, 111.

[219] Whitmore, B. C. 2002, in: A Decade of Hubble Space Telescope Science, M. Livio, K. Noll, M. Stiavelli, Eds. (Cambride Univ. Press, Cambridge, 2002), pp. 153-180.

[220] Whitney, B. A., Wood, K., Bjorkman, J. E., \& Wolff, M. J. 2003, ApJ, 591, 1049.

[221] Whittet, D. C. B., Gerakines, P. A., Hough, J. H., \& Shenoy, S. S. 2001, ApJ, 547, 872.

[222] Wilke, K., Klaas, U., Lemke, D., Mattila, K., Stickel, M., \& Haas, M. 2004, A\&A, 414, 69.

[223] Wisniewski, J. P., Bjorkman, K. S., Bjorkman, J. E., \& Clampin, M. 2007, ApJ, 670, 1331. 
[224] Wood, D. O. S. \& Churchwell, E. 1989, ApJ, 340, 265.

[225] Wood, K., Whitney, B. A., Robitaille, T., \& Draine, B. T. 2008, ApJ, 688, 1118.

[226] Wood, K., Barnes, J. E., Ercolano, B., Haffner, L. M., Reynolds, R. J., \& Dale, J. 2013, ApJ, accepted. arXiv:1304.1689.

[227] Wünsch, R., Silich, S., Palouš, J., Tenorio-Tagle, G. 2007, A\&A, 471, 579.

[228] Wünsch, R., Teonorio-Tagle, G., Palouš, J., \& Silich, S. 2008, AJ, 135, 2222.

[229] Wu, Y., Charmandaris, V., Hao, L., Brandl, B. R., Bernard-Salas, J., Spoon, H. W. W., \& Houck, J. R. 2006, ApJ, 639, 157.

[230] Wu, J., Evans, N. J., Shirley, Y. L., \& Knez, C. 2010, ApJS, 188, 313.

[231] Yao, L., Bell, T. A., Viti, S., Yates, J. A., \& Seaquist, E. R. 2006, ApJ, 636, 881.

[232] Yeh, S. C. C., Verdolini, S., Krumholz, M. R., Matzner, C. D., \& Tielens, A. G. G. M. 2013, ApJ, accepted. arXiv:1303.4742.

[233] Zheng, Z., Cen, R., Trac., H., Miralda-Escudé, J., \& Weinberg, D. 2011, Galaxy Formation: An International Conference. P.198. 